ELECTRON TRANSPORT WITHIN THE WURTZITE AND ZINC-BLENDE PHASES OF GALLIUM NITRIDE AND INDIUM NITRIDE

by

Poppy Siddiqua

M.Sc., North South University, 2011

A THESIS SUBMITTED IN PARTIAL FULFILLMENT OF

THE REQUIREMENTS FOR THE DEGREE OF

DOCTOR OF PHILOSOPHY

in

THE COLLEGE OF GRADUATE STUDIES

(Electrical Engineering)

THE UNIVERSITY OF BRITISH COLUMBIA

(Okanagan)

March 2019

() Poppy Siddiqua, 2019 
The following individuals certify that they have read, and recommend to the College of Graduate Studies for acceptance, a thesis/dissertation entitled:

\section{ELECTRON TRANSPORT WITHIN THE WURTZITE AND ZINC-BLENDE PHASES OF} GALLIUM NITRIDE AND INDIUM NITRIDE

submitted by Poppy Siddiqua in partial fulfillment of the requirements of

the degree of Doctor of Philosophy

Dr. Stephen K. O'Leary, School of Engineering

\section{Supervisor}

Dr. Jonathan Holzman, School of Engineering

\section{Supervisory Committee Member}

Dr. Murray Neuman, Irving K. Barber School of Arts and Sciences

\section{Supervisory Committee Member}

Dr. Jake Bobowski, Irving K. Barber School of Arts and Sciences

\section{University Examiner}

Dr. Jayshri Sabarinathan, Department of Electrical and Computer Engineering, The University of Western Ontario

\section{External Examiner}




\begin{abstract}
Wide energy gap semiconductors are broadly recognized as promising materials for novel electronic and opto-electronic device applications. As informed device design requires a firm grasp on the material properties of the underlying electronic materials, the electron transport that occurs within the wide energy gap semiconductors has been the focus of considerable study over the years. We review analyses of the electron transport within some wide energy gap semiconductors of current interest. In this thesis, I primarily focus on the electron transport that occurs within the wurtzite and zinc-blende phases of gallium nitride and indium nitride, these materials being of great current interest to the wide energy gap semiconductor community; indium nitride, while not a wide energy gap semiconductor of itself, is included as it is often alloyed with other wide energy gap semiconductors. The electron transport that occurs within zinc-blende gallium arsenide has also been considered. Most of the discussion focus on the steady-state and transient electron transport results obtained from the ensemble semi-classical three-valley Monte Carlo simulations of the electron transport within these materials. The evolution of the field, a survey of the current literature, and some applications for the results will also be featured. Based on this analysis, we have drawn the following conclusions. First, it is found that all of the velocityfield characteristics corresponding to the materials under investigation in this analysis exhibit peaks, regions of negative differential mobility, and regions of high-field saturation. Wurtzite Indium nitride, with its small electron effective mass, exhibits the highest peak electron drift velocity. The transient overshoot observed for the case of wurtzite Indium nitride is also found to be the most pronounced of all of the materials considered in this analysis. This suggests that the wurtzite phase of Indium nitride and its zinc-blende counterpart may offer great potential for future electron device applications.
\end{abstract}




\section{Lay Summary}

The study of electron transport within semiconductors has a long and rich tradition. In this thesis, I present recently acquired electron transport results, corresponding to the wurtzite and zincblende phases of gallium nitride and indium nitride, materials that have attracted a considerable following in recent years. Both steady-state and transient electron transport results are featured, these being obtained from ensemble Monte Carlo simulations. The zinc-blende phase results represent some of the first detailed examinations into the nature of the electron transport within these materials. Other results, such as those corresponding to the wurtzite phases, and those corresponding to gallium arsenide, are added in for benchmarking purposes. Projections for device performance, based on these results, are offered. In addition to a presentation of results, the evolution of the field, a survey of the current literature, and some applications for the results presented herein, will also be featured. 


\section{Preface}

At the outset, I would like to make it clear that while others have certainly contributed to the overall body of work presented within the scope of this thesis, the majority of the work was performed by me and by me alone. In addition to learning about the background required for the work, my work really began with a tabulation of the material and band structural parameters corresponding to the wurtzite and zinc-blende phases of gallium nitride and indium nitride. Given the changes that have been occurring in the understanding of these materials, this was not a completely straightforward matter. I then sequentially went through each material, performing both steady-state and transient electron transport simulations for each material under the range of conditions considered in this thesis. I then used a routine in order to extract the relevant electron transport properties associated with each simulation. From these raw results, figures were generated and critical comparisons were made.

As a consequence of this body of work, six journal papers, four conference proceedings, and one book chapter were published; further details are discussed in Appendix A. The majority of these publications focused on the nature of the electron transport within the wurtzite and zincblende phases of gallium nitride and indium nitride, results corresponding to the nature of the electron transport within zinc oxide also being published during the course of my studies. With editorial corrections from my academic advisor, Dr. Stephen K. O'Leary, I wrote the text associated with each manuscript. I also produced the figures associated with each manuscript. So the majority of the content related to these publications was authored by me. Copyright permissions have been sought and acquired from the respective publishers.

In terms of my co-authors, they have been involved with Monte Carlo simulations of semiconductor materials for many years. Their role in these publications that I have published, however, was more as supporting authors rather than as principal ones. Dr. Michael S. Shur of 
Rensselaer Polytechnic Institute, for example, was one of the first researchers to explore the wide energy gap semiconductors, GaN and InN, through the use of Monte Carlo electron transport simulations. In fact, some of his initial explorations into the nature of the electron transport within these materials were performed by him in concert with Dr. O'Leary. Dr. Shur and Dr. O'Leary have established a vigorous and active research collaboration, and the papers that I wrote within the scope of my doctoral studies were merely the latest of a large number of publications that have arisen from this collaboration. Dr. Shur commented on each manuscript, and made some suggestions in terms of interpreting the results. These definitely helped improve the clarity and effectiveness of my publications. In terms of my other co-authors, i.e., Dr. Brian Foutz, Dr. Walid A. Hadi, Dr. Lester F. Eastman, and Mr. Amith K. Salhotra, they usually played relatively minor supporting roles as former collaborators of my academic advisor, Dr. O'Leary. Dr. Hadi and Mr. Salhotra were former students of Dr. O'Leary, while Dr. Eastman (now deceased) and Dr. Foutz were longer-term research collaborators of Dr. O'Leary.

A final note that is worth mentioning and that is related to the two review articles that I published during the course of my graduate studies. These review articles are the latest in a series of review articles that have been published by my supervisor. Structurally, they are very similar, although different results, corresponding to different materials, are presented. For the last two review articles, I was the first and primary author, the results presented being from my simulations of the electron transport within the wurtzite and zinc-blende phases of these materials. The structure of the last review article was that employed for the purposes of this dissertation. 


\section{Table of Contents}

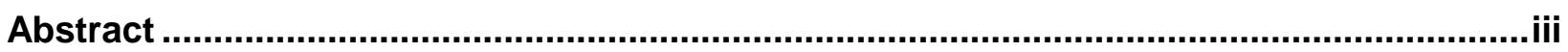

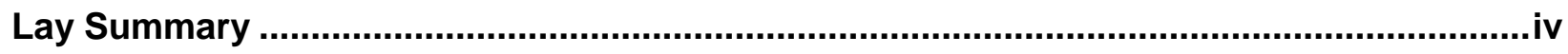

Preface

Table of Contents.........................................................................................................

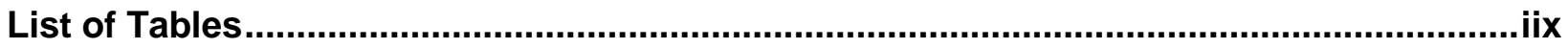

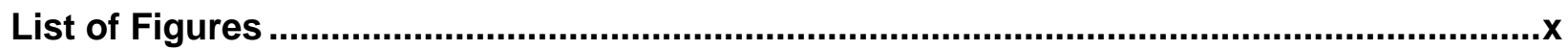

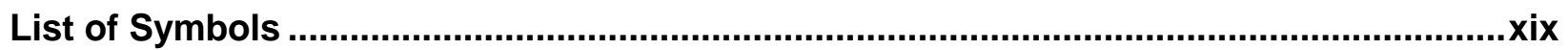

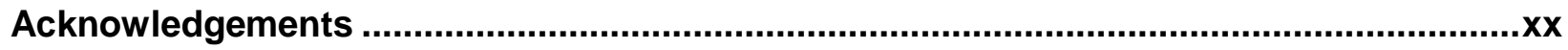

Dedication

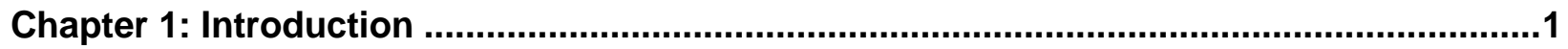

Chapter 2: Electron transport within semiconductors .........................................................11

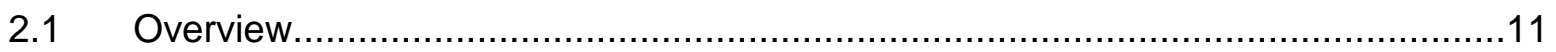

2.2 Electron transport in the continuum limit: the Boltzmann transport equation ..........13

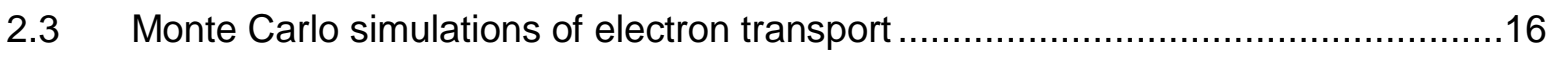

2.4 The ensemble Monte Carlo simulation algorithm …......................................17

2.5 Parameter selections for the wurtzite and zinc-blende phases of $\mathrm{GaN}$ and $\mathrm{InN}$.....33

2.6 The Monte Carlo simulation approach ………...............................................

2.7 Scattering rates in the wurtzite and zinc-blende phases of $\mathrm{GaN}$ and $\operatorname{InN}$..............44

Chapter 3: Steady-state and transient electron transport results within the wurtzite and

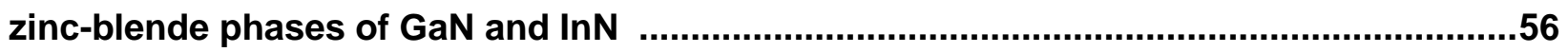

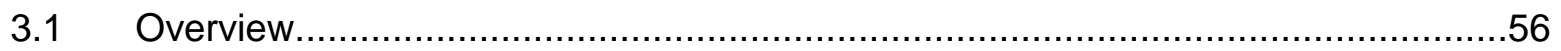

3.2 Steady-state electron transport within wurtzite GaN........................................57

3.3 Steady-state electron transport within zinc-blende GaN....................................63

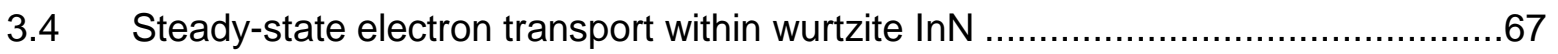

3.5 Steady-state electron transport within zinc-blende $\operatorname{lnN} \ldots \ldots \ldots \ldots \ldots \ldots \ldots \ldots \ldots \ldots \ldots \ldots . . . .68$ 
3.6 Steady-state electron transport within zinc-blende GaAs

3.7 Steady-state electron transport: a comparison of the results associated with the wurtzite and zinc-blende phases of $\mathrm{GaN}$ and $\mathrm{InN}$ with those associated with zinc-blende GaAs .81

3.8 The sensitivity of the velocity-field characteristics associated with wurtzite GaN, zincblende $\mathrm{GaN}$, wurtzite $\operatorname{lnN}$, zinc-blende $\ln N$, and zinc-blende $\mathrm{GaAs}$ to variations in the crystal temperature. .82

3.9 The sensitivity of the velocity-field characteristics associated with wurtzite GaN, zincblende GaN, wurtzite InN, zinc-blende InN, and zinc-blende GaAs to variations in the doping concentrations .98

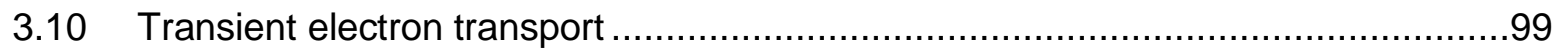

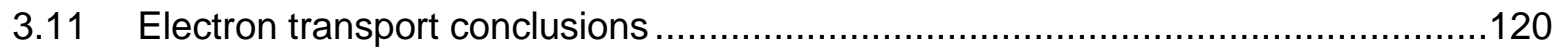

Chapter 4: Electron transport within wide energy gap semiconductors: A review .........121

4.1 The study of electron transport within the family of wide energy gap semiconductors 121

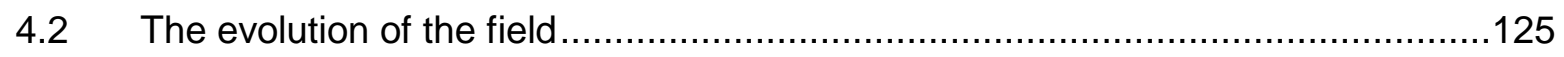

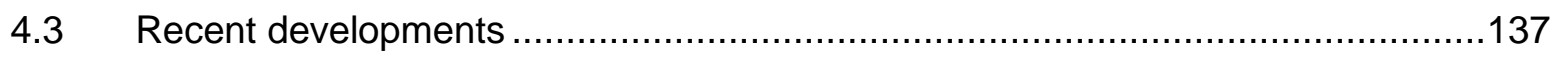

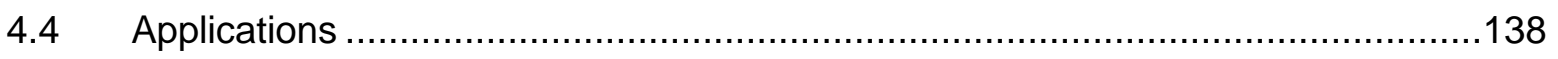

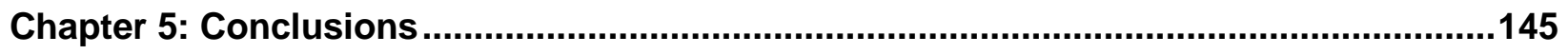

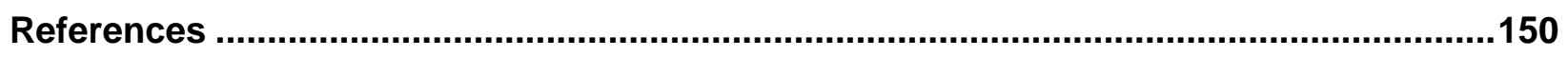

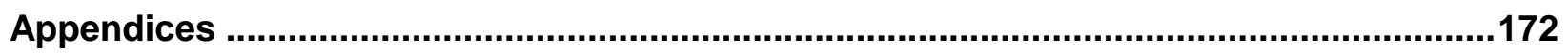

Appendix A: Published work while at The University of British Columbia .......................172

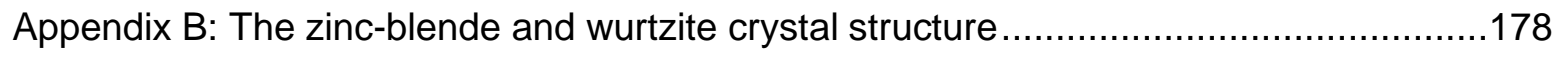

Appendix C: Further details related to my Monte Carlo algorithm .............................181 


\section{List of Tables}

Table 2.1 The material parameter selections corresponding to the wurtzite and zinc-blende phases of GaN and InN. 199

Table 2.2 The band structural parameter selections corresponding to the wurtzite and zinc-blende phases of GaN and InN.

Table 2.3 The material and band structural parameters, corresponding to the other common compound semiconductors considered in this analysis. 36

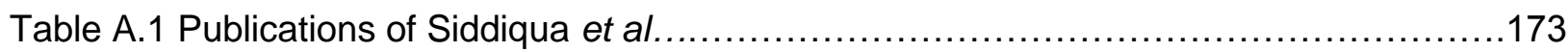




\section{List of Figures}

\section{Chapters}

Figure 1.1: Number of published papers per year corresponding to $\mathrm{GaN}$ and $\ln \mathrm{N}$ contrasted with those exclusively focused on the zinc-blende phases of these materials.

Figure 2.1: A flowchart corresponding to the electron transport Monte Carlo simulation algorithm .20

Figure 2.2.a: The three-valley model used to represent the conduction band electron band structure associated with bulk wurtzite GaN for the Monte Carlo simulations of the electron transport within this material. .25

Figure 2.2.b: The three-valley model used to represent the conduction band electron band structure associated with bulk zinc-blende GaN for the Monte Carlo simulations of the electron transport within this material. .26

Figure 2.2.c: The three-valley model used to represent the conduction band electron band structure associated with bulk wurtzite $\operatorname{lnN}$ for the Monte Carlo simulations of the electron transport within this material.

Figure 2.2.d: The three-valley model used to represent the conduction band electron band structure associated with bulk zinc-blende InN for the Monte Carlo simulations of the electron transport within this material.

Figure 2.2.e: The three-valley model used to represent the conduction band electron band structure associated with bulk zinc-blende GaAs for the Monte Carlo simulations of the electron transport within this material. 
Figure 2.3: The energy gap, $E_{g}$, at $300 \mathrm{~K}$, for a number of elemental and compound semiconductors.

Figure 2.4: The electron effective mass associated with the lowest energy valley as a function of the $E_{0}$ energy gap, at $300 \mathrm{~K}$, for the semiconductors considered in this analysis and other compound semiconductors 38

Figure 2.5: The polar optical phonon energy as a function of the $E_{0}$ energy gap, at $300 \mathrm{~K}$, for the semiconductors considered in this analysis and other compound semiconductors. 39

Figure 2.6: The static relative dielectric constant as a function of the $E_{0}$ energy gap, at $300 \mathrm{~K}$, for the semiconductors considered in this analysis and other compound semiconductors.

Figure 2.7: The high-frequency relative dielectric constant as a function of the $E_{0}$ energy gap, at $300 \mathrm{~K}$, for the semiconductors considered in this analysis and other compound semiconductors

Figure 2.8: The breakdown field strength as a function of the $E_{0}$ energy gap, at $300 \mathrm{~K}$, for the semiconductors considered in this analysis and other compound semiconductors

Figure 2.9.a: The scattering rates for the lowest energy $(Г)$ valley as a function of the magnitude of the wave-vector for bulk wurtzite GaN

Figure 2.9.b: The scattering rates for the lowest energy $(\ulcorner)$ valley as a function of the magnitude of the wave-vector for bulk wurtzite GaN .47

Figure 2.10.a: The scattering rates for the lowest energy $(\ulcorner)$ valley as a function of the magnitude of the wave-vector for bulk zinc-blende GaN. 48 
Figure 2.10.b: The scattering rates for the lowest energy $(\ulcorner)$ valley as a function of the magnitude of the wave-vector for bulk zinc-blende GaN.

Figure 2.11.a: The scattering rates for the lowest energy $(\ulcorner)$ valley as a function of the magnitude of the wave-vector for bulk wurtzite $\ln N$. .50

Figure 2.11.b: The scattering rates for the lowest energy $(\ulcorner)$ valley as a function of the magnitude of the wave-vector for bulk wurtzite $\ln N$.

Figure 2.12.a: The scattering rates for the lowest energy $(\ulcorner)$ valley as a function of the magnitude of the wave-vector for bulk zinc-blende $\ln \mathrm{N}$. .52

Figure 2.12.b: The scattering rates for the lowest energy $(\ulcorner)$ valley as a function of the magnitude of the wave-vector for bulk zinc-blende $\ln \mathrm{N}$. .53

Figure 2.13.a: The scattering rates for the lowest energy $(\ulcorner)$ valley as a function of the magnitude of the wave-vector for bulk zinc-blende GaAs. .54

Figure 2.13.b: The scattering rates for the lowest energy $(\Gamma)$ valley as a function of the magnitude of the wave-vector for bulk zinc-blende GaAs. .55

Figure 3.1: The velocity-field characteristic associated with bulk wurtzite GaN for the crystal temperature set to $300 \mathrm{~K}$ and the doping concentration set to $10^{17} \mathrm{~cm}^{-3}$........ .60

Figure 3.2: The average electron energy as a function of the applied electric field strength for bulk wurtzite GaN for crystal temperature set to $300 \mathrm{~K}$ and doping concentration set to $10^{17} \mathrm{~cm}^{-3}$ 61 
Figure 3.3: The valley occupancy as a function of the applied electric field strength for the case of bulk wurtzite GaN for the crystal temperature set to $300 \mathrm{~K}$ and the doping concentration set to $10^{17} \mathrm{~cm}^{-3}$

Figure 3.4: The velocity-field characteristic associated with bulk zinc-blende GaN for the crystal temperature set to $300 \mathrm{~K}$ and the doping concentration set to $10^{17} \mathrm{~cm}^{-3}$. .64

Figure 3.5: The average electron energy as a function of the applied electric field strength for bulk zinc-blende GaN for the crystal temperature set to $300 \mathrm{~K}$ and the doping concentration set to $10^{17}$ $\mathrm{cm}^{-3}$ .65

Figure 3.6: The valley occupancy as a function of the applied electric field strength for the case of bulk zinc-blende GaN for the crystal temperature set to $300 \mathrm{~K}$ and the doping concentration set to $10^{17} \mathrm{~cm}^{-3}$ .66

Figure 3.7:The velocity-field characteristic associated with bulk wurtzite $\ln N$ for the crystal temperature set to $300 \mathrm{~K}$ and the doping concentration set to $10^{17} \mathrm{~cm}^{-3}$.

Figure 3.8: The average electron energy as a function of the applied electric field strength for bulk wurtzite $\operatorname{InN}$ for the crystal temperature set to $300 \mathrm{~K}$ and doping concentration set to $10^{17} \mathrm{~cm}^{-3} . .70$

Figure 3.9: The valley occupancy as a function of the applied electric field strength for the case of bulk wurtzite $\mathrm{InN}$ for the crystal temperature set to $300 \mathrm{~K}$ and the doping concentration set to $10^{17} \mathrm{~cm}^{-3}$ .71

Figure 3.10: The velocity-field characteristic associated with bulk zinc-blende $\ln \mathrm{N}$ for the crystal temperature set to $300 \mathrm{~K}$ and the doping concentration set to $10^{17} \mathrm{~cm}^{-3}$. .74 
Figure 3.11: The average electron energy as a function of the applied electric field strength for bulk zinc-blende InN for the crystal temperature set to $300 \mathrm{~K}$ and the doping concentration set to $10^{17} \mathrm{~cm}^{-3}$ .75

Figure 3.12: The valley occupancy as a function of the applied electric field strength for the case of bulk zinc-blende InN for the crystal temperature set to $300 \mathrm{~K}$ and the doping concentration set to $10^{17} \mathrm{~cm}^{-3}$. .76

Figure 3.13: The velocity-field characteristic associated with bulk zinc-blende GaAs for the crystal temperature set to $300 \mathrm{~K}$ and the doping concentration set to $10^{17} \mathrm{~cm}^{-3}$. .78

Figure 3.14: The average electron energy as a function of the applied electric field strength for bulk zinc-blende GaAs for the crystal temperature set to $300 \mathrm{~K}$ and the doping concentration set to $10^{17} \mathrm{~cm}^{-3}$. .79

Figure 3.15: The valley occupancy as a function of the applied electric field strength for the case of bulk zinc-blende GaAs for the crystal temperature set to $300 \mathrm{~K}$ and the doping concentration set to $10^{17} \mathrm{~cm}^{-3}$. .80

Figure 3.16.a: A comparison of the velocity-field characteristics associated with the wurtzite and zinc-blende phases of $\mathrm{GaN}$ and $\mathrm{InN}$.

Figure 3.16.b: A comparison of the velocity-field characteristics associated with the wurtzite and zinc-blende phases of $\mathrm{GaN}$ and $\mathrm{InN}$, with that associated with zinc-blende GaAs.... .84

Figure 3.16.c: A comparison of the peak and saturation electron drift velocities associated with the wurtzite and zinc-blende phases $\mathrm{GaN}$ and $\operatorname{lnN}$, with that associated with zinc-blende GaAs. .85 
Figure 3.16.d: A comparison of the peak electric fields, i.e., the electric field strengths at which the peak electron drift velocities occur, associated with the wurtzite and zinc-blende phases of GaN and InN, with that associated with zinc-blende GaAs. .86

Figure 3.17.a: The velocity-field characteristics associated with bulk wurtzite GaN for various crystal temperatures. .88

Figure 3.17.b: The peak and saturation electron drift velocities associated with bulk wurtzite GaN as a function of the crystal temperature. .89

Figure 3.18.a: The velocity-field characteristics associated with bulk zinc-blende GaN for various crystal temperatures. .90

Figure 3.18.b: The peak and saturation electron drift velocities associated with bulk zinc-blende GaN as a function of the crystal temperature. .91

Figure 3.19.a: The velocity-field characteristics associated with bulk wurtzite $\ln N$ for various crystal temperatures. .92

Figure 3.19.b: The peak and saturation electron drift velocities associated with bulk wurtzite $\ln N$ as a function of the crystal temperature. .93

Figure 3.20.a: The velocity-field characteristics associated with bulk zinc-blende InN for various crystal temperatures. .94

Figure 3.20.b: The peak and saturation electron drift velocities associated with bulk zinc-blende InN as a function of the crystal temperature. .95

Figure 3.21.a: The velocity-field characteristics associated with bulk zinc-blende GaAs for various crystal temperatures. .96 
Figure 3.21.b: The peak and saturation electron drift velocities associated with bulk zinc-blende GaAs as a function of the crystal temperature. 97

Figure 3.22.a: The velocity-field characteristics associated with bulk wurtzite GaN for various doping concentrations. 100

Figure 3.22.b: The peak and saturation electron drift velocities associated with bulk wurtzite GaN as a function of the doping concentration 101

Figure 3.23.a: The velocity-field characteristics associated with bulk zinc-blende GaN for various doping concentrations. 102

Figure 3.23.b: The peak and saturation electron drift velocities associated with bulk zinc-blende GaN as a function of the doping concentration. 103

Figure 3.24.a: The velocity-field characteristics associated with bulk wurtzite $\ln N$ for various doping concentrations. 104

Figure 3.24.b: The peak and saturation electron drift velocities associated with bulk wurtzite $\ln N$ as a function of the doping concentration .105

Figure 3.25.a: The velocity-field characteristics associated with bulk zinc-blende $\ln N$ for various doping concentrations. .106

Figure 3.25.b: The peak and saturation electron drift velocities associated with bulk zinc-blende InN as a function of the doping concentration. .107

Figure 3.26.a: The velocity-field characteristics associated with bulk zinc-blende GaAs for various doping concentrations. 108 
Figure 3.26.b: The peak and saturation electron drift velocities associated with bulk zinc-blende GaAs as a function of the doping concentration 109

Figure 3.27: The electron drift velocity as a function of the distance displaced since the application of the electric field, for various applied electric field strength selections, for the case of bulk wurtzite GaN

Figure 3.28: The electron drift velocity as a function of the distance displaced since the application of the electric field, for various applied electric field strength selections, for the case of bulk zincblende GaN. 113

Figure 3.29: The electron drift velocity as a function of the distance displaced since the application of the electric field, for various applied electric field strength selections, for the case of bulk wurtzite $\ln N$ 114

Figure 3.30: The electron drift velocity as a function of the distance displaced since the application of the electric field, for various applied electric field strength selections, for the case of bulk zincblende $\ln N$ 115

Figure 3.31: The electron drift velocity as a function of the distance displaced since the application of the electric field, for various applied electric field strength selections, for the case of bulk zincblende GaAs. 116

Figure 3.32.a: A comparison of the velocity overshoot amongst the bulk semiconductors considered in this analysis, i.e., the wurtzite and zinc-blende phases of $\mathrm{GaN}$ and $\operatorname{InN}$, with that associated with bulk zinc-blende GaAs .118 
Figure 3.32.b: A comparison of the peak transient electron drift velocities associated with the bulk semiconductors considered in this analysis, i.e., the wurtzite and zinc-blende phases of GaN and InN, with that associated with bulk zinc-blende GaAs. 119

Figure 4.1: The low-field electron mobility of wurtzite GaN plotted as a function of the crystal temperature

Figure 4.2: The cut-off frequency and the optimal operating voltage plotted as functions of the device length-scale, $L$, for the cases of wurtzite GaN, zinc-blende GaN, wurtzite InN, and zincblende $\ln N$ 144

Figure 5.1: The cut-off frequency plotted as a function of the device length-scale, $L$, for the case of wurtzite GaN. 148

\section{Appendices}

Figure A.1: The number of articles in the field compared with that of O'Leary et al. and Siddiqua et al., for the specific case of GaN. .175

Figure A.2: The number of articles in the field compared with that of O'Leary et al. and Siddiqua et al., for the specific case of $\ln N$ 176

Figure B.1: The zinc-blende crystal structure. 179

Figure B.2: The wurtzite crystal structure .180

Figure C.1: A more complete flowchart for my Monte Carlo algorithm used for simulating electron transport within the III-V nitride semiconductors, GaN and InN. .182

Figure C.2: The scattering mechanism selection process. .187 


\section{List of Symbols}

Boltzman's constant $\quad k_{B}$

Charge of an electron $q$

Cut-off frequency $\quad f_{t}$

Electric field $\quad \vec{E}$

Electron wave-vector $\quad \vec{k}$

Electron velocity $\vec{v}$

Electron energy $\quad E$

Effective mass of electron $m^{*}$

Energy gap $E_{g}$

Non-parabolicity coefficient $\quad \alpha$

Electron momentum $\quad \vec{P}$

Electron drift mobility $\quad \mu$

Electron transit time $\tau$ 


\section{Acknowledgements}

I am deeply grateful to my Ph.D. supervisor, Dr. Stephen K. O'Leary, for his constant support and scholarly guidance throughout my studies. His helpful suggestions and patience throughout my research have made this dissertation possible.

I would like to thank Dr. Jonathan Holzman and Dr. Murray Neuman for their willingness to serve as my committee members. I truly appreciate their time and constructive comments on my research work.

I extend my gratitude for all the assistance and encouragement given to me by my one and only sister, Dr. Sumi Siddiqua. Without her inspiration and support, none of my achievements would be possible.

A special thanks to my mom, Mamtaz Begum, for looking after the kids while I was busy with my research for days and nights.

I would like to thank all the fellow graduate students who helped me with their constructive feedback during my writing process.

Finally, a special thanks are owed to my husband, Arefin, and daughters, Ayesha and Sayibah. I thank Arefin for always being there for me and supporting me with all of my decisions. 


\section{Dedication}

Dedicated to my mother. Mamtag Begum. A strong and pure soul who taught me to have faith in Allah, believe in hard work and be in right path. 


\section{Chapter 1: Introduction}

The family of III-V nitride semiconductors, which includes gallium nitride (GaN), aluminum nitride (AIN), and indium nitride $(\operatorname{lnN})$, has long been recognized as a promising material system for novel electronic and opto-electronic device applications [1-14]. The wide energy gaps associated with some of these materials, and their alloys, suggests that they will exhibit high breakdown fields, high thermal conductivities, and favorable electron transport characteristics, making them ideally suited for high-power and high-frequency device applications [15-25]. The direct nature of the energy gaps associated with some of the compounds present within the III-V nitride semiconductor group [26,27] implies that these materials are also well-suited for novel opto-electronic device applications over a broad range of the electromagnetic spectrum, much broader than that offered by some of the other commonly encountered group III-V semiconductor alloy systems [28-39].

Work on the III-V nitride semiconductors, which initially focused upon the binary compounds GaN, AIN, and InN, found its genesis in the earlier parts of the $20^{\text {th }}$ Century [40-45]. Pioneering forms of these materials, small crystals and powders, were of poor-quality, and thus, unsuitable for device applications. Unfortunately, the growth difficulties, which plagued initial efforts at working with these materials, persisted for many years thereafter, hindering efforts at developing III-V nitride semiconductor-based technologies [46,47]. Eventually, however, the research effort invested paid off, and high-quality forms of the III-V nitride semiconductors became available for device applications $[48,49]$. Much of the electron device work performed since then has focused on the use of the III-V nitride semiconductors for high-frequency, highpower, high-temperature, and high-radiation device applications, where traditional semiconductors, such as silicon (Si) and gallium arsenide (GaAs), prove inadequate [50-56]. III$\mathrm{V}$ nitride semiconductor opto-electronic device work has primarily focused on the use of such materials in optical devices, such as lasers and light-emitting diodes, operating in the infra-red- 
to-ultraviolet region of the electromagnetic spectrum, a broad and technologically significant region not served by traditional families of opto-electronic materials [57-61]. These developments have sparked considerable interest into the III-V nitride semiconductors.

The wurtzite and zinc-blende crystal structures are the most commonly encountered phases found for binary compound semiconductors; a tutorial on the wurtzite and zinc-blende crystal structures is provided in Appendix B of this thesis. Thermodynamic arguments have demonstrated that wurtzite is GaN's stable phase [62]. It is now widely recognized, however, that the zinc-blende phase of this material, which can be prepared through the careful deposition of GaN onto an appropriately selected cubic substrate, offers a number of distinct advantages over its wurtzite form. In particular, zinc-blende GaN offers higher carrier mobilities, higher carrier drift velocities, and a higher doping efficiency than its wurtzite counterpart [63-68]. The greater symmetry inherent to the zinc-blende crystal structure leads to the absence of spontaneous polarization induced-electric fields, thereby making devices fabricated from this material less prone to failure [69-71]. The fact that zinc-blende GaN cleaves more easily than its wurtzite counterpart further adds to its allure, suggesting easier device processing and hinting at opportunities for integration with $\mathrm{Si}$ and GaAs-based technologies $[68,69,72]$. Prodded on by some pioneering work aimed at depositing this particular form of GaN [73-78], zinc-blende GaN's device potential has recently attracted some attention from researchers within the III-V nitride semiconductor community $[79,80]$.

Research has demonstrated that $\operatorname{InN}$ also presents itself in the wurtzite and zinc-blende forms. As with the case of GaN, thermodynamic arguments have demonstrated that wurtzite is InN's stable phase. As a consequence, most research aimed at characterizing the material properties of InN has been concentrated on the wurtzite phase of this material. It should be noted, however, that some research into the zinc-blende phase $\operatorname{InN}$ has also been performed. Initial reports into zinc-blende InN include those of Chandrasekhar et al. [81], Jenkins et al. [82], Strite 
et al. [83], Tebata et al. [84], and Yamamoto et al. [85]. These studies have demonstrated that, as with the case of $\mathrm{GaN}$, zinc-blende $\mathrm{InN}$ may be prepared through the deposition of InN onto an appropriately selected cubic substrate under certain conditions. The unique properties of zincblende $\operatorname{InN}$ have led some to speculate that this particular form of InN may be more useful for certain device applications than its wurtzite counterpart.

As the wurtzite phases of $\mathrm{GaN}$ and $\operatorname{InN}$ are the stable phases, the majority of work that has been performed within the III-V nitride semiconductor community has focused on these phases. This has effectively led to the neglect of the zinc-blende phases of GaN and InN. The paucity of results pertaining to the zinc-blende phases of $\mathrm{GaN}$ and $\operatorname{lnN}$ may be directly demonstrated, for example, in Figure 1.1, in which a bar chart contrasts the number of papers published corresponding to $\mathrm{GaN}$ and $\ln \mathrm{N}$ against those exclusively focused on the zinc-blende phases of these materials. ${ }^{1}$ Given the focus on the wurtzite phases of GaN and $\operatorname{lnN}$, it is clear that many aspects pertaining to the zinc-blende phases of these materials remain unknown. This is a lamentable state of affairs if one aims to employ the zinc-blende phases of these materials for device applications.

1 The Web of Science was employed for the purposes of this analysis. For the identification of papers broadly focused on GaN and InN, the search terms "gallium nitride" and "indium nitride", respectively, were employed. For the identification of papers focused only on the zinc-blende phases of these materials, the search terms "gallium nitride zinc blende" and "indium nitride zinc blende" were employed. It should be noted, however, that there may be some flaws associated with this methodology. For example, papers that deposit GaN on a cubic substrate, even if the GaN itself is not in the zinc-blende phase, would be inadvertently included in the counts as a paper focused on the zinc-blende phase of GaN. Given that these results are purely meant to demonstrate the primacy of studies focused on the wurtzite phases of GaN and $\operatorname{InN}$, the possibility of such errors is acceptable. 


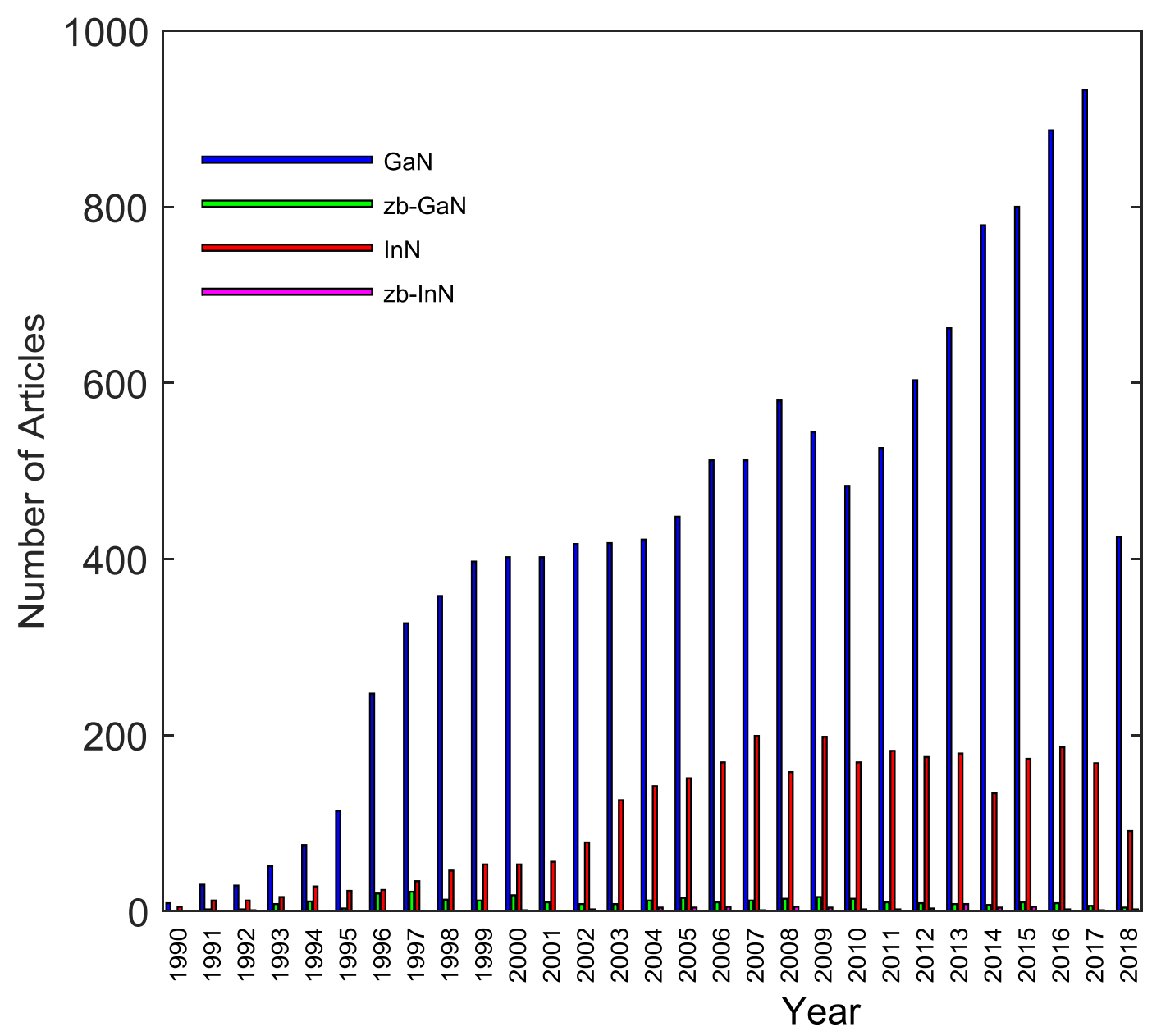

Figure 1.1: Number of published papers per year corresponding to $\mathrm{GaN}$ and $\mathrm{InN}$ contrasted with those exclusively focused on the zinc-blende phases of these materials. The methodology that was employed for the purposes of this particular aspect of the analysis is discussed in footnote 1 . The online version of this figure is in color. 
There is now a widespread recognition that informed semiconductor device design requires a detailed understanding of the underlying electron transport processes. In a given device structure, the electrons that are present will respond to the application of an electric field. The motion of these electrons, which in large measure determines the performance of such a device, is the fundamental issue at stake in the study of electron transport. Owing to its important role in shaping device performance, understanding the electron transport that occurs within semiconductors, and developing tools whereby the mechanisms underlying it may be quantitatively characterized and related to semiconductor device performance, has been a high priority for semiconductor device engineers for many years [86], and continues to be so today $[87,88]$. Through this understanding, improvements in semiconductor device performance have been achieved and a framework for device optimization has been developed.

The electron transport response, i.e., how an ensemble of electrons responds to the application of an electric field, has two components: (1) steady-state and (2) transient. Steadystate electron transport may be characterized through the specification of transport characteristics that are achieved long after all of transients are extinguished. Transient electron transport, by way of contrast, provides a detailed analysis as to how these steady-state values are achieved, i.e., the extinction of these transients is the primary focus of studies into the transient electron response. While the majority of electron transport studies have focused on examining the steadystate response, in more recent years the transient response has become a greater focus of study. This has occurred as a consequence of the recognition that enhancements in device performance may be acquired through transient effects. For example, electrons transiting across a short device may not have the ability to acquire steady-state before they exit the device. In the analysis presented in my thesis, both steady-state and transient electron transport responses are examined.

Prior to my work, the electron transport processes that occur within the zinc-blende phases of $\mathrm{GaN}$ and $\mathrm{InN}$ remained poorly understood. Electron transport within the zinc-blende phase of 
GaN had only been studied by a couple of authors [63,64,69,81-85,89-93]. Most of these analyses focused only on the steady-state component of the electron transport response, the transient electron transport response remaining unknown. The study of electron transport within the zincblende phase of $\operatorname{InN}$ had only been attended to by only one previous author [94]. Given the important role that electron transport plays in defining the potential of a given material for device applications, it is clear that further work is required if the widely recognized device potential of the zinc-blende phases of GaN and $\mathrm{InN}$ is to be fully realized.

Recognizing this critical deficiency in the scientific literature, in collaboration with my academic advisor at The University of British Columbia, Dr. Stephen K. O'Leary, I aimed to ameliorate it. First, I assembled all of the scientific articles that I could find focused on electron transport within the wurtzite and zinc-blende phases of $\mathrm{GaN}$ and $\ln \mathrm{N}$ that were available at the time. ${ }^{2}$ Then I identified what aspects of electron transport remained unresolved, with a particular focus on the less understood materials, i.e., the zinc-blende phases of $\mathrm{GaN}$ and $\operatorname{lnN}$. A number of publications, representing original research, arose as a consequence of this body of work, the aim of each publication being discussed in Appendix A. While most of the papers that I participated in were focused on the wurtzite and zinc-blende phases of GaN and InN, some other electron transport results were also published during the course of my doctoral studies.

By the time I had published my last original research article, I recognized that the collection of articles that I had authored, along with my supervisor, formed the state-of-the-art in terms of what is currently known in terms of electron transport within the zinc-blende phases of GaN and InN. Accordingly, given our prominent role in the field, we were invited by a reviews editor to submit a review on our latest electron transport results corresponding to the zinc-blende phases of GaN and InN. In order to make the work more complete, we also reviewed analyses of

\footnotetext{
${ }^{2}$ For the purpose of this analysis, I solely focused on electron transport results that were acquired through the use of Monte Carlo electron transport simulations.
} 
the electron transport within the wurtzite phases of $\mathrm{GaN}$ and $\operatorname{lnN}$, these results also being in accord with the state-of-the-art. So I completed this body of work with the publication of a review article in 2018, entitled "Electron transport within the wurtzite and zinc-blende phases of gallium nitride and indium nitride", which was published in the Journal of Materials Science: Materials in Electronics. This article surveys the state-of-the-art in terms of what is currently known about the electron transport within the wurtzite and zinc-blende phases of $\mathrm{GaN}$ and $\operatorname{lnN}$. It features a tutorial on electron transport and on the Monte Carlo approach that may be used for its quantitative characterization, a presentation of state-of-the-art electron transport results corresponding to the wurtzite and zinc-blende phases of $\mathrm{GaN}$ and $\operatorname{lnN}$, a description of the evolution of the field, and a presentation of some applications of the results. Some potential topics for future research are provided at the conclusion of this review. This review article, in large measure, provides the organizational framework for this thesis. Copyright permissions, corresponding to all of the articles that I published during the course of my doctoral studies, have been sought and acquired from all of the respective publishers.

In this thesis, material and band structural parameters, corresponding to the various materials under investigation, i.e., the wurtzite and zinc-blende phases of GaN and InN, were assembled and Monte Carlo simulations of the steady-state and transient components of the electron transport response, were determined, the acquired results being analysed and critically contrasted with each other. While modifications to the Monte Carlo code were not pursued in of themselves, all of the electron transport simulations and subsequent data processing was performed by me. I have adopted an unorthodox approach to the presentation of my results. Rather than first presenting the sequence of critical developments that have transpired as the electron transport results have evolved into their current form, I first present the state-of-the-art results themselves for the different materials considered in this analysis, i.e., the wurtzite and zinc-blende phases of $\mathrm{GaN}$ and $\mathrm{InN}$. I then present how these electron transport results have evolved into their current form. Most doctoral dissertations, by way of contrast, present the 
sequence of historical developments first and then feature the state-of-the-art results that have been acquired. The reasons for the adoption of this particular organizational structure for this particular thesis are two-fold. First and foremost, my original research contributions, and by extension, those presented by others in this rapidly evolving field, are, if presented from a purely chronological perspective, seemingly disjointed and fragmented. They address particular aspects of the electron transport that remain unclear to experts in the field, not providing much in the way of context for the significance of the presented results. This is because scientific articles are presented with the technical expert in mind, not the technical lay-person, this being the focus of a thesis. Secondly, the chief challenge presented by this thesis work was the assembly of the material and band structural parameters and the use of the Monte Carlo simulation approach in the characterization of the electron transport response corresponding to the materials under consideration, i.e., the wurtzite and zinc-blende phases of $\mathrm{GaN}$ and $\operatorname{lnN}$. In a sense, electron transport results from the past, in of themselves, with the exception of the updates to the materials and band structural parameter selections, played very little role in shaping the course of my thesis work, and therefore, were not really relevant background material. Accordingly, the thesis is structured with a background chapter, focused on electron transport within semiconductors, a chapter presenting the state-of-the-art results for all of the materials under consideration in this analysis, and a chapter that provides some insight in terms of how the current state-of-the-art consensus has evolved.

As electron transport lies at the heart of this thesis, it begins with a general discussion on the basic principles underlying the electron transport that occurs within semiconductors. The Boltzmann transport equation, ${ }^{3}$ which allows for the quantitative description of the electron trans-

\footnotetext{
${ }^{3}$ The Boltzmann transport equation quantitatively describes how an ensemble of electrons responds to the application of an electric field. It provides for a quantitative characterization of electron transport. It is further discussed in Chapter 2.
} 
port within a semiconductor, is introduced as a corollary. Then, the Monte Carlo electron transport simulation approach, and how it may be employed in order to characterize electron transport within a semiconductor, are detailed. Material and band structural parameters, corresponding to the semiconductors under investigation in this analysis, i.e., the wurtzite and zinc-blende phases of $\mathrm{GaN}$ and $\mathrm{InN}$, are then presented; the wurtzite phases are included in the consideration for comparative purposes. A critical comparison, with the parameter selections corresponding to other compound semiconductors of interest, is also featured. Steady-state and transient Monte Carlo electron transport simulation results, corresponding to the materials under investigation in this analysis, i.e., the wurtzite and zinc-blende phases of $\mathrm{GaN}$ and $\operatorname{InN}$, are then presented. The thesis concludes by examining what is currently known about the electron transport within the IIIV nitride compound semiconductors, GaN, AIN, and InN, and related materials, describes how this understanding has evolved into its current form, and presents a number of applications for the results presented herein. Bulk electron transport within the materials under investigation, i.e., the wurtzite and zinc-blende phases of $\mathrm{GaN}$ and $\mathrm{InN}$, will be the principal focus of this analysis. Results corresponding to GaAs will also be presented, albeit primarily for bench-marking purposes.

This thesis is organized in the following manner. In Chapter 2, the mechanisms underlying electron transport within semiconductors are introduced, and the use of Monte Carlo electron transport simulations, in the characterization of the electron transport within semiconductors, is discussed. The material and band structural parameters, corresponding to the wurtzite and zincblende phases of GaN and InN, are also featured in Chapter 2, these parameters being used in the subsequent analysis. A critical comparison, between these material and band structural parameters and those corresponding to other compound semiconductors of interest, is also provided. Steady-state and transient electron transport results, obtained from our ensemble semiclassical three-valley Monte Carlo electron transport simulations within the wurtzite and zinc- 
blende phases of $\mathrm{GaN}$ and $\mathrm{InN}$, are then presented in Chapter 3. What is currently known about the electron transport within the broader community of wide energy gap compound semiconductors of interest, i.e., GaN, AIN, and InN, and related materials, how this understanding has evolved into its current form, and a number of applications for the results presented herein, are then discussed in Chapter 4. Finally, the conclusions of this thesis are drawn in Chapter 5. 


\section{Chapter 2: Electron transport within semiconductors}

\section{$2.1 \quad$ Overview}

Device performance is a primary consideration for a semiconductor device designer. The electron transport processes that occur within a semiconductor are known to play a critical role in determining this performance. Accordingly, understanding the electron transport that occurs within semiconductors, and developing tools whereby the mechanisms underlying it may be quantitatively characterized and related to semiconductor device performance, has been a high priority for semiconductor device designers for many years [95], and continues to be so today $[96,97]$. Through this understanding, improvements in semiconductor device performance have been achieved.

The electrons within a semiconductor are in a perpetual state of motion. In the absence of an applied electric field, this motion arises as a result of the thermal energy which is present, and is referred to as thermal motion. From the perspective of an individual electron, thermal motion may be viewed as a series of trajectories, interrupted by a series of random scattering events. Scattering may arise as a result of interactions with the lattice atoms, impurities, other electrons, and defects. As these interactions lead to electron trajectories in all possible directions, i.e., there is no preferred direction, while individual electrons will move from one location to another, taken as an ensemble, assuming that the electrons are in thermal equilibrium, the overall electron distribution will remain static. Accordingly, no net current flow occurs.

With the application of an applied electric field $\vec{E}$, each electron in the ensemble will experience a force, $-q \vec{E}$, where $q$ denotes the magnitude of the electron charge. While this force may have a negligible impact upon the motion of any given individual electron, taken as an ensemble, the application of such an electric field will lead to a net aggregate motion of the electron distribution. Accordingly, a net current flow will occur, and the overall electron ensemble 
will no longer be in thermal equilibrium. Understanding how the electron ensemble evolves in response to the application of an electric field, in essence, represents the fundamental issue at stake when the electron transport within a semiconductor is studied [95].

In this chapter, I start with a brief primer on the nature of the electron transport within semiconductors and on the Monte Carlo electron transport simulation approach, and then detail the Monte Carlo electron transport simulations employed for the purposes of this particular analysis. Initially, I discuss how an ensemble of electrons may be treated as a continuum in phasespace. I then introduce the Boltzmann transport equation, and discuss how it may be used in order to examine, in the continuum limit, the evolution of an ensemble of electrons within phasespace under the action of an applied electric field. The principles underlying Monte Carlo simulation analyses of the electron transport within semiconductors are then presented, and an explanation as to how this approach may be used in order to solve the Boltzmann transport equation, is provided. I then introduce the ensemble semi-classical three-valley Monte Carlo simulation approach, used in the simulations of the electron transport within the wurtzite and zincblende phases of $\mathrm{GaN}$ and $\mathrm{InN}$. The material and band structural parameter selections, corresponding to the wurtzite and zinc-blende phases of $\mathrm{GaN}$ and $\ln N$, are then presented. A critical comparison with the material and band structural parameters corresponding to other compound semiconductors of interest is also provided. An overview of the Monte Carlo simulation approach that I adopt for the purposes of this analysis is then presented. Finally, the dependence of the scattering rates on the magnitude of the electron wave-vector, $\vec{k}$ corresponding to the key scattering processes, is provided for the cases of the wurtzite and zinc-blende phases of GaN and $\ln N$.

This chapter is organized in the following manner. In Section 2.2, the treatment of an electron ensemble as a continuum in phase-space, and the use of the Boltzmann transport equation, in order to determine the evolution of this ensemble under the action of an applied 
electric field, is presented. Then, in Section 2.3, the basic principles underlying Monte Carlo simulations of electron transport, and how such an approach yields a solution to the Boltzmann transport equation, is discussed. Details, related to the Monte Carlo algorithm employed, and various approximations that are often introduced, are then provided in Section 2.4. In Section 2.5, the material and band structural parameter selections, corresponding to the wurtzite and zincblende phases of $\mathrm{GaN}$ and $\operatorname{InN}$, are presented, a critical comparison with the material and band structural parameters corresponding to other compound semiconductors also being provided. The specifics of the ensemble semi-classical three-valley Monte Carlo simulation approach to treating electron transport within a semiconductor are then featured in Section 2.6. Finally, the dependence of the scattering rates on the magnitude of the electron wave-vector, $\vec{k}$, for the key scattering processes shaping the nature of the electron transport within a compound semiconductor, is provided for the materials considered in this analysis, i.e., the wurtzite and zincblende phases of $\mathrm{GaN}$ and $\operatorname{lnN}$, in Section 2.7. Analogous results, corresponding to the specific case of zinc-blende GaAs, are also depicted in Section 2.7.

\subsection{Electron transport in the continuum limit: the Boltzmann transport equation}

For the case of a bulk semiconductor, the individual nature of the electrons within an ensemble can be neglected. Instead, these electrons may be considered as being part of a continuum. In this continuum limit, the distribution of electrons may be characterized through the specification of the distribution function, $f(\vec{r}, \vec{k}, t), f(\vec{r}, \vec{k}, t) \Delta r \Delta k$ representing the number of electrons within the infinitesimal volume $\Delta r \Delta k$ around the point $(\vec{r}, \vec{k})$ in phase-space at time $t, \vec{r}$ denoting the position and $\vec{k}$ representing the electron wave-vector; in this context, phase-space corresponds to the direct product of the real and electron wave-vector spaces. The Boltzmann transport equation provides a quantitative means of determining how this distribution function evolves with time under the action of an external applied electric field. 
The distribution function corresponding to an electron ensemble within a semiconductor evolves in time owing to three basic driving factors. There is the transfer of electrons in real-space, owing to the electron velocities that occur. There is the transfer of electrons in electron wavevector space, these being related to the rate of change, with respect to time, of the electron wavevector associated with the different electrons. Finally, there are the scattering processes that occur. Analytically, Shur [96] expresses the Boltzmann transport equation as

$$
\frac{\partial f(\vec{r}, \vec{k}, t)}{\partial t}=-\vec{v} \cdot \nabla_{r} f(\vec{r}, \vec{k}, t)-\dot{\vec{k}} \cdot \nabla_{k} f(\vec{r}, \vec{k}, t)+\left.\frac{\partial f(\vec{r}, \vec{k}, t)}{\partial t}\right|_{\text {scat }},
$$

where the terms following the equal sign in Eq. (2.1), from left to right, correspond to the three aforementioned driving factors; $\vec{v}$ corresponds to the rate of change of $\vec{r}$, i.e., $\vec{v}=\dot{\vec{r}}$. That is, while the first term corresponds to the transfer of electrons in real-space, owing to the electron velocities that occur, the second term corresponds to the transfer of electrons in electron wave-vector space, owing to the rate of change, with respect to time, of the electron wave-vectors associated with the different electrons, the final term corresponding to the contributions related to the different scattering processes that occur. Fundamentally, the Boltzmann transport equation is a continuity equation for the distribution function. Its solution provides for a complete characterization of the nature of the electron transport within a bulk semiconductor. Further discussion on the Boltzmann transport equation is provided in the literature $[98,99]$.

Owing to its critical importance in understanding the nature of the electron transport within semiconductors, a number of solutions to the Boltzmann transport equation have been devised over the years. Low-field asymptotic analytical solutions include those of Chin et al. [100], Shur et al. [101], and Look et al. [102]. Higher-field approximate analytical solutions include those of Ferry [1], Das and Ferry [2], Conwell and Vassel [103], Sandborn et al. [104], and Zukotynski and Howlett [105]. Numerical techniques, that solve the Boltzmann transport equation directly, have 
also been developed. Unfortunately, the numerical computations that are demanded of such an approach are extremely intense, and typically, one must make approximations in order to allow for numerical tractability. These techniques are further discussed by Nag [95].

The electron transport that occurs within a semiconductor has both steady-state and transient components. Accordingly, the solution to the Boltzmann transport equation must take into account both aspects of the electron transport response. In considering steady-state electron transport, the state of the distribution function long after the application of the electric field, i.e., after all of the transients have been fully extinguished, is considered. ${ }^{4}$ In contrast, when considering transient electron transport, how the distribution function evolves in time is considered. Both the steady-state and transient components of electron transport, corresponding to the wurtzite and zinc-blende phases of $\mathrm{GaN}$ and $\mathrm{InN}$, are considered in this thesis.

As was mentioned previously, the Boltzmann transport equation applies to cases for which the corpuscular nature of the electrons within an ensemble may be neglected. For the case of simulating the electron transport within a bulk semiconductor, as in this analysis, the dimensions of the material are large and the treatment of an ensemble of electrons as a continuum is justifiable. When the dimensions are small, however, and quantum effects are significant, then the Boltzmann transport equation, and its continuum treatment of the electron ensemble, is incorrect. For such cases, quantum electron transport approaches must be employed instead for the treatment of the electron transport. Such approaches lie beyond the scope of the work reviewed here but are adequately discussed in the literature [106].

${ }^{4}$ Once all of the transients have been fully extinguished, the electron ensemble will have shifted into a new equilibrium state. By an equilibrium state, however, we are not necessarily referring to thermal equilibrium, thermal equilibrium only being achieved in the absence of an applied electric field. 


\subsection{Monte Carlo simulations of electron transport}

In a Monte Carlo simulation of electron transport, instead of treating the ensemble of electrons as a continuum, as in the Boltzmann transport equation, one instead focuses on the electron transport of the individual electrons within the ensemble. Through tracking the motion of an individual electron for a long time, or through simulating the motion of a large number of electrons, a solution to the Boltzmann transport equation will emerge in the continuum limit, i.e., as the time elapsed in the simulation becomes long or as the number of electrons considered in the simulation becomes large. The accuracy of this solution will increase as the continuum limit is approached, i.e., as the length of time of the simulation is increased or as the number of electrons in the simulation is increased. Underlying both electron transport simulation approaches, a random number generator is used in order to simulate the random character of the electron transport within a semiconductor. This electron transport simulation approach is frequently employed in the simulation of the electron transport within semiconductors. With specific reference to the compound semiconductors being considered in this particular analysis, i.e., the wurtzite and zinc-blende phases of $\mathrm{GaN}$ and $\operatorname{lnN}$, Monte Carlo simulations of the electron transport are employed for the analysis of these materials within the scope of this thesis.

As was hinted at earlier, there are actually two broad categories of Monte Carlo simulation that can be employed in the analysis of electron transport: (1) single-particle, and (2) ensemble. In a single-particle Monte Carlo simulation, the transit of an individual electron is examined. Given sufficient time, the amount of time spent in any particular region of phase-space will be proportional to the distribution function, $f(\vec{r}, \vec{k}, t)$, there. In contrast, for an ensemble Monte Carlo simulation, the transit of a large number of electrons is considered. In this approach, the number of electrons at any particular region in phase-space will be proportional to the distribution function, $f(\vec{r}, \vec{k}, t)$, there. Assuming that the electron motion is ergodic, i.e., that the time averages correspond to the statistical averages, the resultant distribution functions should be identical [107]. 
Unfortunately, while single-particle Monte Carlo simulations are perfectly capable of resolving the steady-state electron transport, the resolution of the transient electron transport using this technique represents a challenge, variations in time being used as a proxy for ensemble variations in the single-particle approach. ${ }^{5}$ The ensemble Monte Carlo simulation technique, however, allows for the effective treatment of both the steady-state and the transient electron transport responses. Given that I am considering both aspects of the electron transport within the scope of this review, I focus on ensemble Monte Carlo simulations for the purposes of the analysis of the electron transport within the wurtzite and zinc-blende phases of GaN and InN.

\subsection{The ensemble Monte Carlo simulation algorithm}

\subsubsection{Algorithm}

Within the framework of an ensemble Monte Carlo simulation of the electron transport within a semiconductor, the motion of a large number of electrons within the semiconductor, under the action of an applied electric field, is considered. Electrons will transit through the semiconductor, accelerating under the action of the applied electric field, these accelerations being interrupted by scattering processes, these being related to the interaction of the transiting electrons with the thermal motion of the lattice, i.e., phonons, ionized impurities, lattice dislocations, and other electrons. Typically, each type of scattering is characterized by a scattering rate, the probability of a given "scattering event" occurring over an infinitesimal time interval being directly proportional to the product of the corresponding scattering rate and the duration of this time-interval. Clearly, for a Monte Carlo electron transport simulation, the accuracy

\footnotetext{
${ }^{5}$ In a single-particle electron transport simulation, time is being used as a proxy for variations across the ensemble. In a transient electron transport simulation, other properties are varying as a function of time, i.e., the electric field is abruptly turning on, for example. This means that time can not be used as a proxy for ensemble variations, and therefore, the use of the single-particle approach is invalid.
} 
of the results will be shaped, in large measure, by the selection of the scattering rates employed. It is often the case that these scattering rates are determined at the outset of the simulation, however, more sophisticated techniques have been developed which depend upon the properties of the current electron distribution. These scattering rate formulas can be implemented using a self-consistent ensemble technique. This technique recalculates the scattering rate table at regular intervals throughout the simulation as the electron distribution evolves. This selfconsistent ensemble Monte Carlo technique is employed by me for the purposes of this analysis. ${ }^{6}$ The material parameter selections corresponding to the wurtzite and zinc-blende phases of GaN and $\operatorname{lnN}$, which are being employed for the Monte Carlo simulations, are tabulated in Table 2.1

The key elements of the Monte Carlo electron transport simulation algorithm are shown in Figure 2.1; a more detailed flowchart is shown in the Appendix C. In the initialization phase, the initial scattering rate tables are determined. Each electron is assigned a specific point in phasespace, i.e., a value of $\vec{r}$ and a value of $\vec{k}$, the initial distribution of electrons within phase-space being in accordance with Fermi-Dirac occupation statistics, i.e., the ensemble of electrons is initially assumed to be in thermal equilibrium. The evolution of this ensemble of electrons, under the action of an applied electric field, is the issue at stake in the study of the electron transport within a semiconductor [95].

I now consider the main body of the algorithm. In this phase, the motion of the electrons in the ensemble is divided into a number of small time-steps, $\Delta t$. Within each time-step, each electron is assumed to accelerate under the action of the applied electric field over a series of

6 A Monte Carlo electron simulation resource, with source code included, may be found at https://nanohub.org/resources/moca. Further information about the Monte Carlo approach itself, beyond that found within the electron transport simulation context may also be found at http://www.codeproject.com/Articles/767997/Parallelised-Monte-Carlo-Algorithms-sharp and http://www.codeproject.com/Articles/32654/Monte-Carlo-Simulation?q=Monte+Carlo+code 
Table 2.1 The material parameter selections corresponding to the wurtzite and zinc-blende phases of GaN and $\operatorname{InN}$. The parameter selections, corresponding to wurtzite GaN, zinc-blende GaN, wurtzite InN, and zinc-blende InN, are drawn from (C) Siddiqua, P. \& O'Leary, S.K. (2018). Electron transport within the wurtzite and zinc-blende phases of gallium nitride and indium nitride, Journal of Materials Science, 29, 3511-3567. Page 3521. Adapted with permission from publisher.

\begin{tabular}{|c|c|c|c|c|}
\hline Parameter & Wurtzite GaN & Zinc-blende GaN & Wurtzite $\ln N$ & Zinc-blende $\operatorname{InN}$ \\
\hline Mass density ( $\mathrm{g} / \mathrm{cm} 3$ ) & 6.15 & 6.15 & 6.81 & 6.81 \\
\hline Longitudinal sound velocity $(\mathrm{cm} / \mathrm{s})$ & $6.56 \times 10^{5}$ & $6.56 \times 10^{5}$ & $6.24 \times 10^{5}$ & $6.24 \times 10^{5}$ \\
\hline Transverse sound velocity $(\mathrm{cm} / \mathrm{s})$ & $2.68 \times 10^{5}$ & $2.68 \times 10^{5}$ & $2.55 \times 10^{5}$ & $2.55 \times 10^{5}$ \\
\hline Acoustic deformation potential $(\mathrm{eV})$ & 8.3 & 8.3 & 7.1 & 7.1 \\
\hline Static dielectric constant & 8.9 & 8.9 & 15.3 & 15.3 \\
\hline High-frequency dielectric constant & 5.35 & 5.35 & 8.4 & 8.4 \\
\hline Effective mass ( $\Gamma 1$ valley) & $0.20 \mathrm{~m}_{\mathrm{e}}$ & $0.15 \mathrm{~m}_{\mathrm{e}}$ & $0.04 \mathrm{~m}_{\mathrm{e}}$ & $0.054 \mathrm{~m}_{\mathrm{e}}$ \\
\hline Piezoelectric constant, $e_{14}\left(\mathrm{C} / \mathrm{cm}^{2}\right)$ & $3.75 \times 10^{-5}$ & $3.75 \times 10^{-5}$ & $3.75 \times 10^{-5}$ & $3.75 \times 10^{-5}$ \\
\hline Direct energy gap (eV) & 3.39 & 3.2 & 0.7 & 0.7 \\
\hline Polar optical phonon energy (meV) & 91.2 & 91.2 & 73.0 & 73.0 \\
\hline Inter-valley deformation potentials $(\mathrm{eV} / \mathrm{cm})$ & $10^{9}$ & $10^{9}$ & $10^{9}$ & $10^{9}$ \\
\hline Inter-valley phonon energies (meV) & 91.2 & 91.2 & 73.0 & 73.0 \\
\hline
\end{tabular}

$m_{e}$ denotes the free electron mass. 


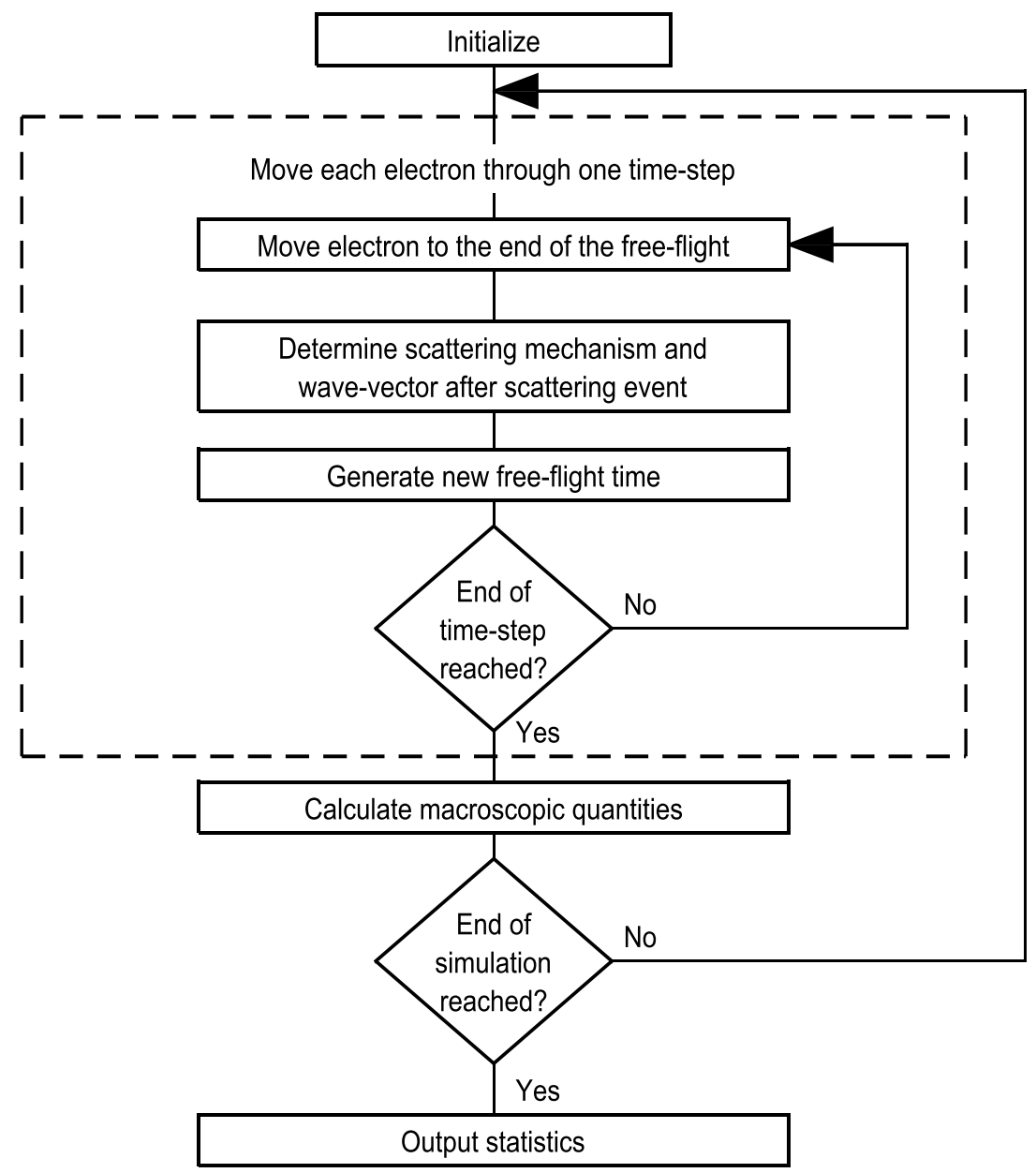

Figure 2.1: A flowchart corresponding to the electron transport Monte Carlo simulation algorithm. A more detailed flowchart is shown in Appendix C. This figure is modified from @ Siddiqua, P., \& O'Leary, S. (2018). Electron transport within the wurtzite and zinc-blende phases of gallium nitride and indium nitride. Journal of Materials Science, 29, 3511-3567. Page 3516. Adapted with permission from publisher. The online version of this figure is depicted in color. 
free-flights, interrupted by a number of scattering events. During each free-flight, an electron experiences no scattering events, and its motion through the conduction band is determined semiclassically. The time for each free-flight must be chosen carefully, and depends critically on the scattering rates at the beginning of the electron's free-flight, as well as the scattering rates throughout its free-flight. Since the scattering rates change over the flight, the selection of the free-flight time is complex. Methods used for generating the free-flight time have been extensively studied, and means of generating free-flight times are further detailed in Appendix C [108]. At the end of each free-flight, the electron experiences a "scattering event." The "scattering event" is chosen randomly, in proportion to the scattering rate for each mechanism. Finally, a new wavevector for the electron is chosen, based on conservation of momentum and conservation of energy considerations, as well as the angular distribution function corresponding to that particular scattering mechanism. After the electron has moved through the free-flight, a new free-flight time is chosen and the process repeats itself until that electron reaches the end of the current timestep.

Once the electrons have gone through a time-step, the resultant electron distribution may be used to determine the electron transport properties of interest. The electron transport properties that I considered in my analysis include the electron drift velocity, ${ }^{7}$ the average electron energy, and the number of electrons in each valley. This process is repeated, time-step after timestep, until the entire simulation is complete. If the results are to be determined as a function of the applied electric field strength, periodic updates to the applied electric field strength selection are performed throughout the simulation, the time interval between updates being sufficient in order to ensure that steady-state is achieved before the next update to the field occurs. Once the simulation is complete, the results are sent to a file for the purposes of archiving, processing, and

\footnotetext{
7 By electron drift velocity, I am referring to the average electron velocity, determined by statistically averaging over the entire electron ensemble.
} 
subsequent retrieval.

\subsubsection{The three-valley model approximation}

Transiting electrons in a semiconductor tend to congregate in the lower energy parts of the conduction band. A great simplification in the analysis may be achieved simply by focusing on the three lowest energy valleys of the conduction band rather than the entire band structure; these "valleys" actually correspond to the regions in $\vec{k}$-space that are in the immediate vicinity of the three lowest energy conduction band minima. A further simplification may be achieved by adopting the Kane model in order to describe the form of each of these three valleys [109]. That is, in the vicinity of each conduction band valley energy minimum, the energy band is taken to be spherically symmetric, and of the form

$$
\frac{\hbar^{2} k^{2}}{2 m^{*}}=E(1+\alpha E),
$$

$\hbar k$ denoting the magnitude of the crystal momentum and $E$ representing the electron energy, $E=$ 0 corresponding to the valley minimum, $m^{*}$ being the effective mass of electrons at the lowest energy point in the valley, and $\alpha$ being the non-parabolicity coefficient associated with that particular valley [110]. ${ }^{8}$ Within the context of the Kane model, this non-parabolicity coefficient,

$$
\alpha=\frac{1}{E_{g}}\left(1-\frac{m^{*}}{m_{e}}\right)^{2},
$$

where $E_{g}$ represents the corresponding energy gap [72]. The three-valley models, used to represent the conduction band structures associated with the wurtzite and zinc-blende phases of $\mathrm{GaN}$ and $\mathrm{InN}$ for the purposes of the Monte Carlo simulations of the electron transport within these materials, are described in Section 2.5 and detailed in Table 2.2. The three-valley models

\footnotetext{
${ }^{8}$ Albrecht et al. [110] generalize this relationship to include a second order non-parabolicity coefficient that reduces to the traditional Kane model, i.e., Eq. (2.2), in the limit that this second-order non-parabolicity coefficient reduces to zero. No dramatic impact on the results is observed.
} 
Table 2.2 The band structural parameter selections corresponding to the wurtzite and zinc-blende phases of $\mathrm{GaN}$ and InN. The parameter selections, corresponding to wurtzite $\mathrm{GaN}$, zinc-blende GaN, wurtzite InN, and zinc-blende InN, are drawn from (C Siddiqua, P. \& O'Leary, S.K. (2018). Electron transport within the wurtzite and zinc-blende phases of gallium nitride and indium nitride, Journal of Materials Science, 29, 3511-3567. Page 3522. Adapted with permission from publisher.

\begin{tabular}{|c|c|c|c|c|}
\hline & Valley number & 1 & 2 & 3 \\
\hline \multirow{6}{*}{ Wurtzite GaN } & Valley location & $\Gamma_{1}$ & $\Gamma_{2}$ & L-M \\
\hline & Valley degeneracy & 1 & 1 & 6 \\
\hline & Effective mass & $0.2 \mathrm{me}_{\mathrm{e}}$ & $m_{e}$ & $\mathrm{me}_{\mathrm{e}}$ \\
\hline & Inter-valley energy separation (eV) & - & 1.9 & 2.1 \\
\hline & Energy gap (eV) & 3.39 & 5.29 & 5.49 \\
\hline & Non-parabolicity $\left(\mathrm{eV}^{-1}\right)$ & 0.189 & 0.0 & 0.0 \\
\hline \multirow{6}{*}{ Zinc-blende GaN } & Valley location & $\Gamma_{1}$ & $\mathrm{x}$ & $\mathrm{L}$ \\
\hline & Valley degeneracy & 1 & 3 & 4 \\
\hline & Effective mass & $0.15 \mathrm{~m}_{\mathrm{e}}$ & $0.40 \mathrm{~m}_{\mathrm{e}}$ & $0.60 \mathrm{~m}_{\mathrm{e}}$ \\
\hline & Inter-valley energy separation (eV) & - & 1.4 & 2.7 \\
\hline & Energy gap (eV) & 3.2 & 4.6 & 5.9 \\
\hline & Non-parabolicity $\left(\mathrm{eV}^{-1}\right)$ & 0.226 & 0.078 & 0.027 \\
\hline \multirow{6}{*}{ Wurtzite $\ln N$} & Valley location & $\Gamma_{1}$ & $\Gamma_{2}$ & L-M \\
\hline & Valley degeneracy & 1 & 1 & 6 \\
\hline & Effective mass & $0.04 \mathrm{~m}_{\mathrm{e}}$ & $0.25 \mathrm{~m}_{\mathrm{e}}$ & $m_{e}$ \\
\hline & Inter-valley energy separation (eV) & - & 1.775 & 2.709 \\
\hline & Energy gap (eV) & 0.70 & 2.475 & 3.409 \\
\hline & Non-parabolicity $\left(\mathrm{eV}^{-1}\right)$ & 1.43 & 0.0 & 0.0 \\
\hline \multirow{6}{*}{ Zinc-blende $\ln N$} & Valley location & $\Gamma_{1}$ & $\mathrm{X}$ & $\mathrm{K}$ \\
\hline & Valley degeneracy & 1 & 3 & 12 \\
\hline & Effective mass & $0.054 \mathrm{me}_{\mathrm{e}}$ & $0.67 m_{e}$ & $0.53 \mathrm{me}_{\mathrm{e}}$ \\
\hline & Inter-valley energy separation (eV) & - & 3.2 & 4.71 \\
\hline & Energy gap (eV) & 0.7 & 3.9 & 5.41 \\
\hline & Non-parabolicity $\left(\mathrm{eV}^{-1}\right)$ & 4.45 & 0.0 & 0.6 \\
\hline
\end{tabular}

$m_{e}$ denotes the free electron mass. 
of the conduction bands corresponding to the materials under investigation in this analysis, i.e., the wurtzite and zinc-blende phases of $\mathrm{GaN}$ and $\mathrm{InN}$, are depicted in Figures 2.2.a-d. The threevalley model of the conduction band associated with zinc-blende GaAs is also depicted in Figure 2.2.e.

\subsubsection{The semi-classical approximation}

An electron wave-function associated with a semiconductor extends across the entire volume of the crystal. Accordingly, at any given instant, a given electron within a semiconductor is able to interact with all of the atoms and all of the other electrons that are present within it. That is, a given electron can simultaneously interact with a multitude of phonons, ionized impurities, lattice dislocations, and other electrons. Unfortunately, this perspective on electron transport is rather complex and does not provide much insight into the character of the electron transport within semiconductors. Thus, it is often the case that assumptions are introduced in order to render the analysis more tractable.

The semi-classical treatment of the motion of electrons within a semiconductor is one of the most common simplifying assumptions that is introduced into analyses of the electron transport within semiconductors. Within the framework of this assumption, each electron within the ensemble is treated as if it were a point particle. From a semi-classical perspective, an electron, with an electron wave-vector, $\vec{k}$, has a velocity

$$
\vec{v}=\frac{1}{\hbar} \nabla_{k} \epsilon(\vec{k})
$$

where $\epsilon(\vec{k})$ denotes the corresponding electron band structure, i.e., the energy of the electron as a function of the electron wave-vector, $\vec{k}$. Under the action of an applied electric field, $\vec{E}$, the rate of change of an electron's momentum, $\vec{p}=\hbar \vec{k}$, with respect to time, may be expressed as

$$
\hbar \frac{d \vec{k}}{d t}=-q \vec{\varepsilon}
$$




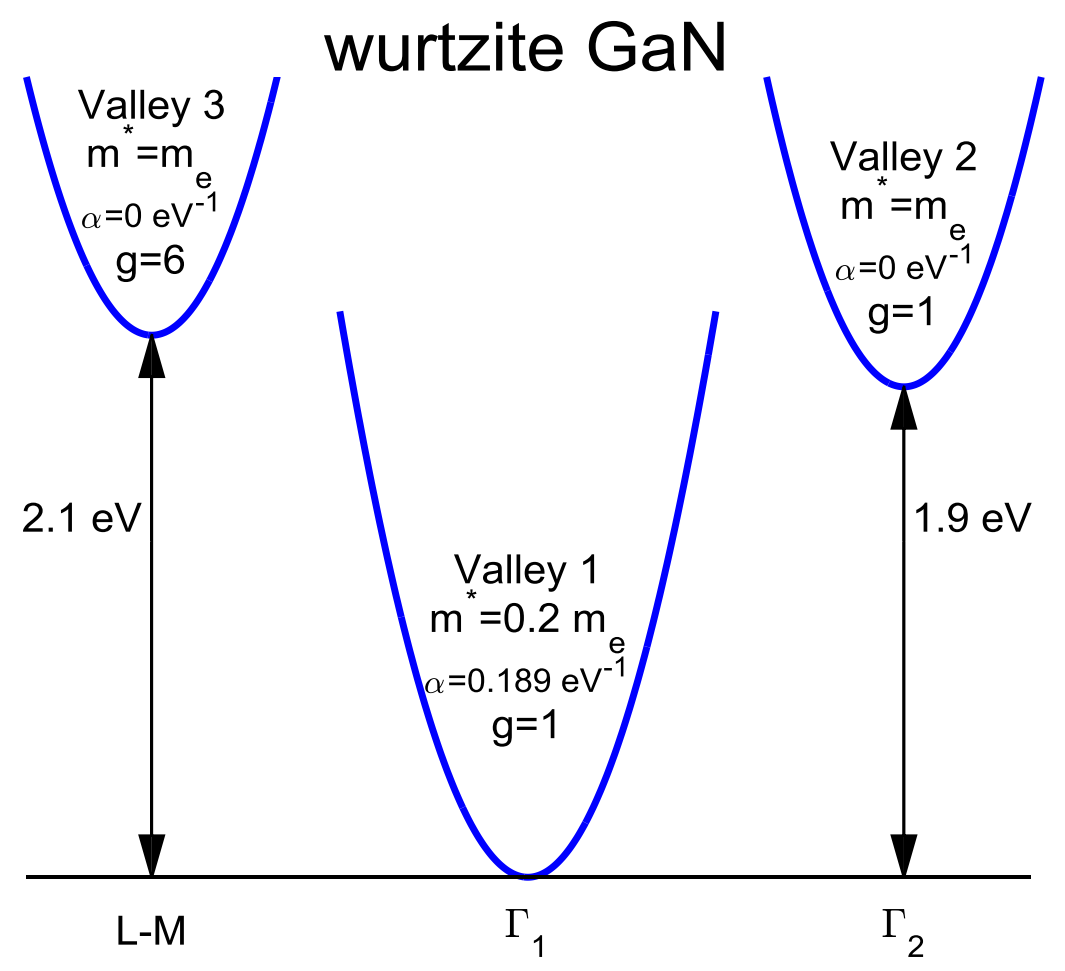

Figure 2.2.a: The three-valley model used to represent the conduction band electron band structure associated with bulk wurtzite GaN for the Monte Carlo simulations of the electron transport within this material. The band structure parameters, corresponding to the bulk wurtzite phase of GaN, are tabulated in Table 2.2; see Section 2.5.1. This figure is modified from @ Siddiqua, P., \& O'Leary, S. (2018). Electron transport within the wurtzite and zinc-blende phases of gallium nitride and indium nitride. Journal of Materials Science, 29, 3511-3567. Page 3518. Adapted with permission from publisher. The online version of this figure is depicted in color. 


\section{zinc-blende GaN}

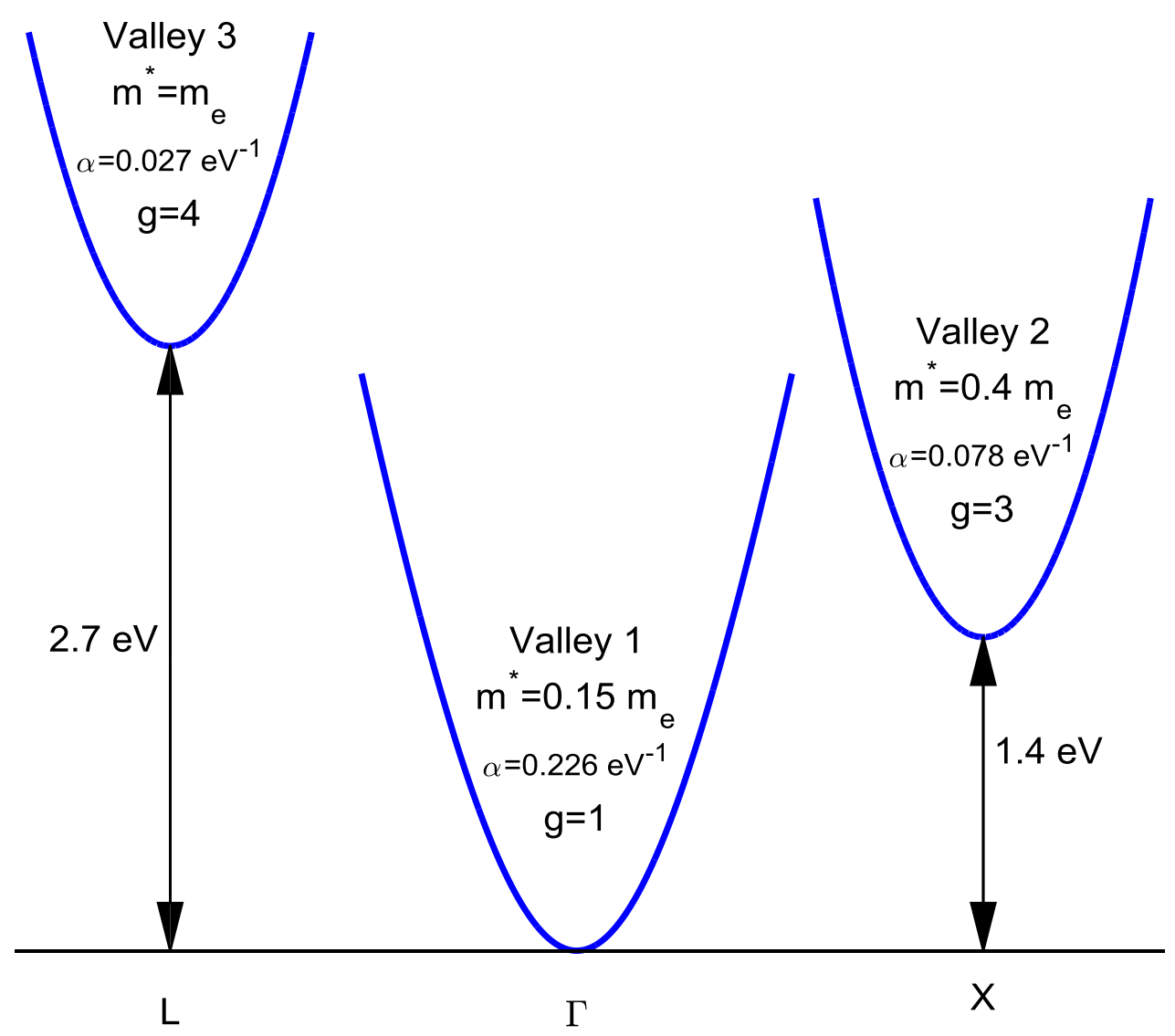

Figure 2.2.b: The three-valley model used to represent the conduction band electron band structure associated with bulk zinc-blende GaN for the Monte Carlo simulations of the electron transport within this material. The band structure parameters, corresponding to the bulk zinc-blende phase of $\mathrm{GaN}$, are tabulated in Table 2.2; see Section 2.5.1. This figure is modified from @ Siddiqua, P., \& O'Leary, S. (2018). Electron transport within the wurtzite and zinc-blende phases of gallium nitride and indium nitride. Journal of Materials Science, 29, 3511-3567. Page 3518. Adapted with permission from publisher. The online version of this figure is depicted in color. 


\section{wurtzite InN}

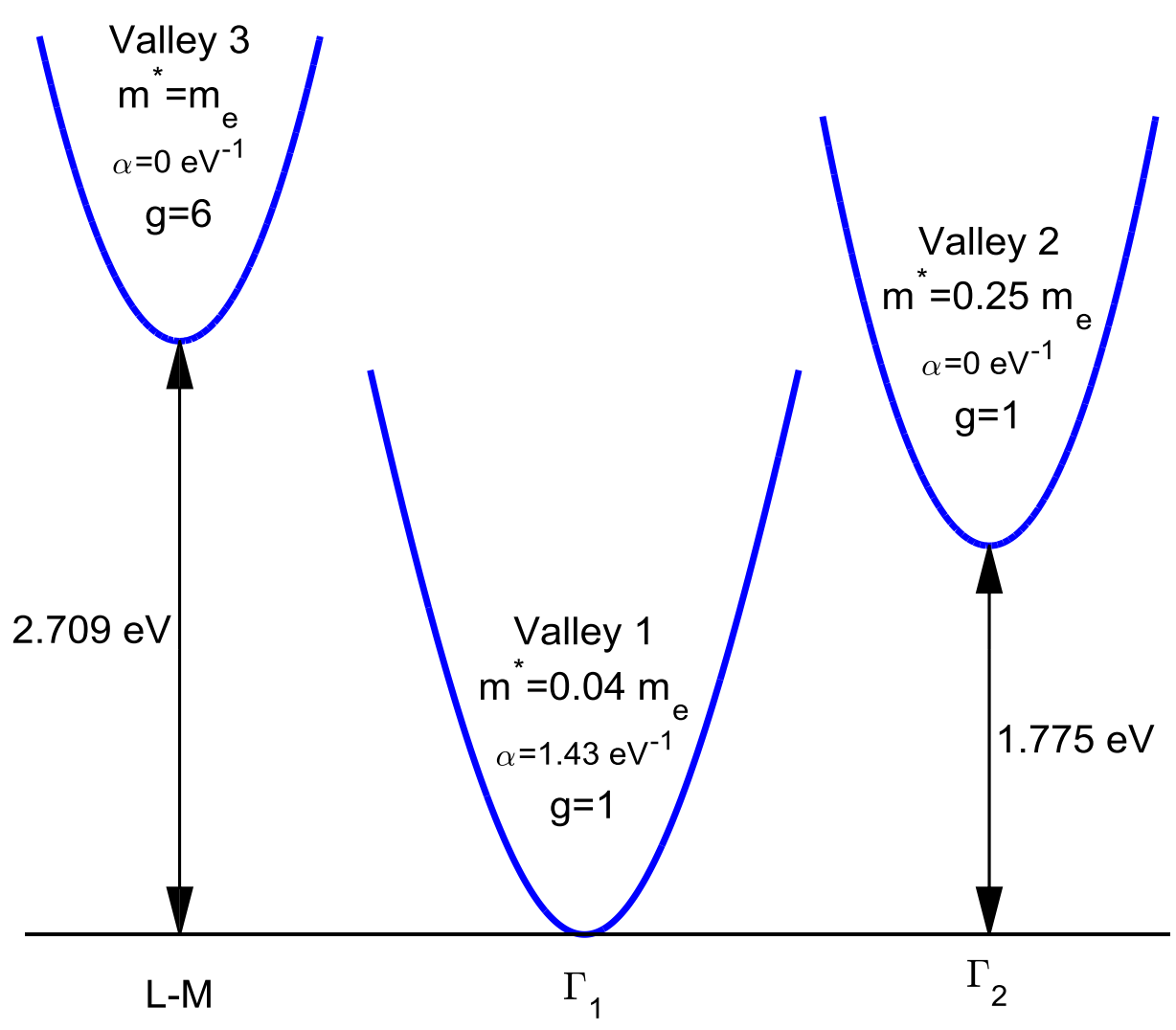

Figure 2.2.c: The three-valley model used to represent the conduction band electron band structure associated with bulk wurtzite $\operatorname{lnN}$ for the Monte Carlo simulations of the electron transport within this material. The band structure parameters, corresponding to the bulk wurtzite phase of $\ln N$, are tabulated in Table 2.2; see Section 2.5.1. This figure is modified from (c Siddiqua, P., \& O'Leary, S. (2018). Electron transport within the wurtzite and zinc-blende phases of gallium nitride and indium nitride. Journal of Materials Science, 29, 3511-3567. Page 3518. Adapted with permission from publisher. The online version of this figure is depicted in color. 


\section{zinc-blende $\ln N$}

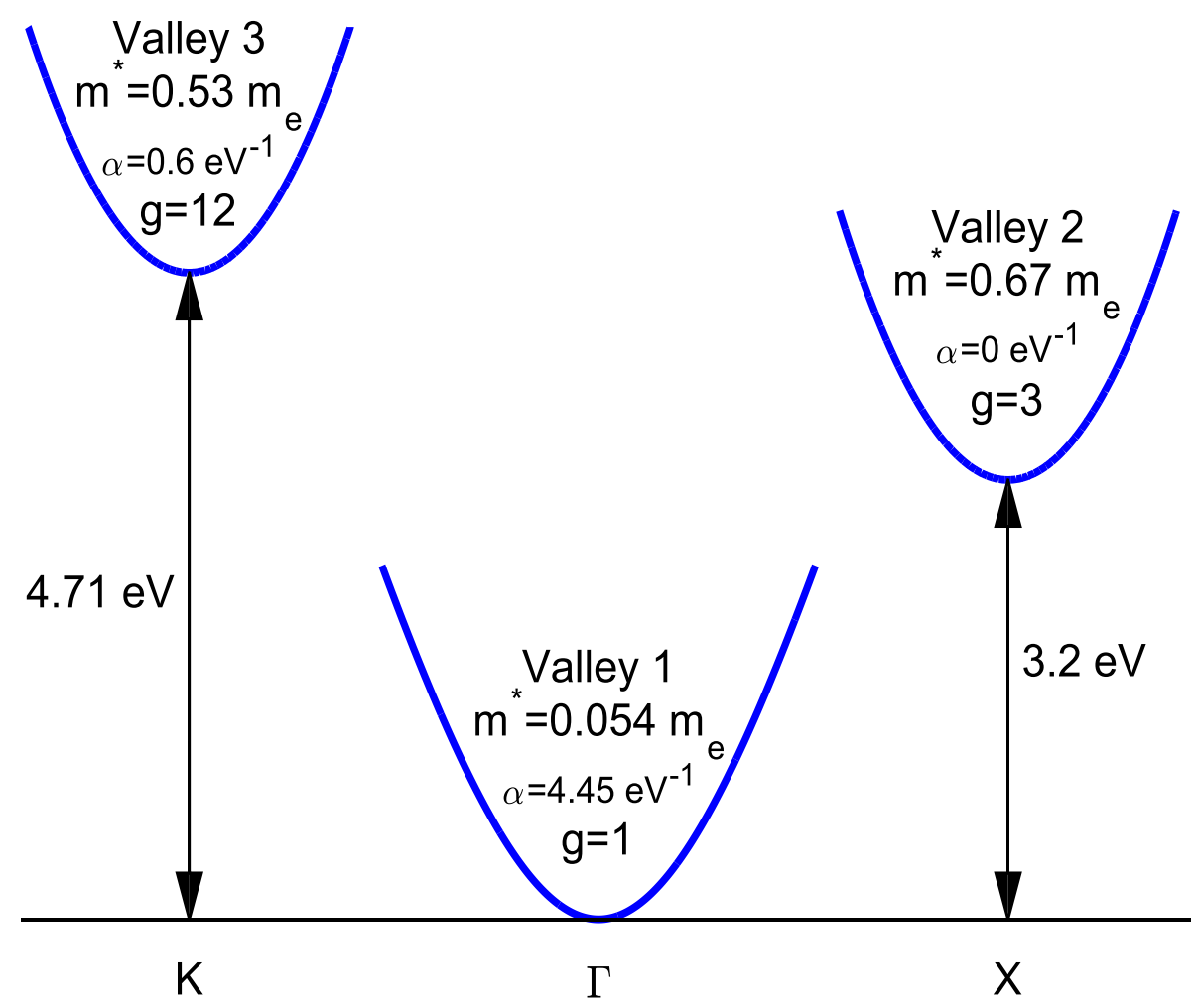

Figure 2.2.d: The three-valley model used to represent the conduction band electron band structure associated with bulk zinc-blende $\operatorname{lnN}$ for the Monte Carlo simulations of the electron transport within this material. The band structure parameters, corresponding to the bulk zinc-blende phase of InN, are tabulated in Table 2.2; see Section 2.5.1. This figure is modified from @ S Siddiqua, P., \& O'Leary, S. (2018). Electron transport within the wurtzite and zinc-blende phases of gallium nitride and indium nitride. Journal of Materials Science, 29, 3511-3567. Page 3518. Adapted with permission from publisher. The online version of this figure is depicted in color. 


\section{zinc-blende GaAs}

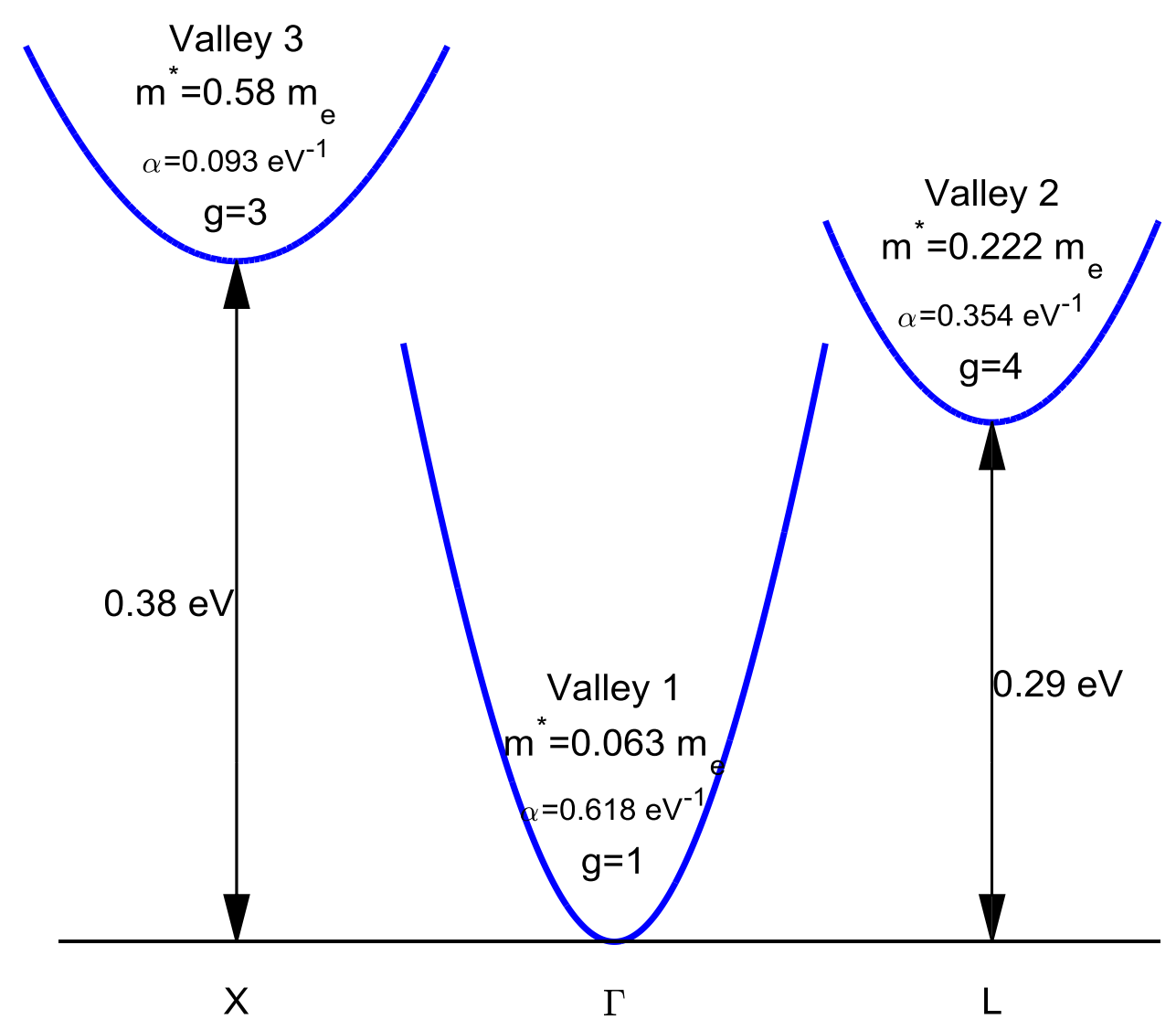

Figure 2.2.e: The three-valley model used to represent the conduction band electron band structure associated with bulk zinc-blende GaAs for the Monte Carlo simulations of the electron transport within this material. This figure is modified from (C Siddiqua, P., \& O'Leary, S. (2018). Electron transport within the wurtzite and zinc-blende phases of gallium nitride and indium nitride. Journal of Materials Science, 29, 3511-3567. Page 3518. Adapted with permission from publisher. The online version of this figure is depicted in color. 
Equations (2.4) and (2.5) define the semi-classical trajectory of this electron, assuming that the periodic potential associated with the underlying crystal is static.

In reality, the thermal motion of the lattice, the presence of ionized imperfections and lattice dislocations, and interactions with the other electrons in the ensemble, result in the electron deviating from the path literally prescribed by the conjuncture of Eqs. (2.4) and (2.5). Although an individual electron's interaction with the lattice is very complex, the description of the ensemble behavior may be considerably simplified through the use of the aforementioned quantum mechanical notion of "scattering events." During a "scattering event," the electron's wave-function abruptly changes. Quantum mechanics determines the probability of each type of "scattering event," and dictates how to probabilistically determine the change in the electron wave-vector after each such event. With this information, the behavior of an ensemble of electrons may be simulated, this behavior being expected to closely approximate the nature of the electron transport within a real semiconductor. The probability of scattering is introduced into the Monte Carlo simulation approach through a determination of the scattering rates corresponding to the different scattering processes.

\subsubsection{Scattering processes}

The scattering rate corresponding to a particular interaction refers to the expected number of "scattering events" of that particular interaction taking place per unit time. Quantum mechanics determines the scattering rates for the different processes based on the physics of the interaction. In general, scattering processes within semiconductors can be classified into three basic types: (1) phonon scattering, (2) defect scattering, i.e., related to lattice dislocations, and (3) carrier scattering [95]. For the semiconductors under investigation in this analysis, i.e., the wurtzite and zinc-blende phases of $\mathrm{GaN}$ and $\operatorname{lnN}$, phonon scattering is the most important scattering mechanism, and it is featured prominently in the simulations of the electron transport within these materials. Defect scattering refers to the scattering of electrons due to the imperfections within the crystal. Throughout this work, it is assumed that donor impurities are the only defects present. 
These defects, when ionized, scatter electrons through their charge. This mechanism is an important factor to consider in determining the electron transport within the wurtzite and zincblende phases of $\mathrm{GaN}$ and $\mathrm{InN}$, and the effect of the doping concentration on the electron transport within these materials is treated within the scope of our analysis. The final category of scattering mechanism, carrier scattering, or in our case, electron-electron scattering, has also been considered for some of the simulations that we have performed. It should be noted, however, that as this scattering mechanism leads to very little change in the results with a substantial increase in the running time, in an effort to determine my results as expeditiously as possible, the results presented herein do not include electron-electron scattering.

Owing to their importance in determining the nature of the electron transport within the semiconductors under investigation in this analysis, i.e., the wurtzite and zinc-blende phases of GaN and $\operatorname{lnN}$, it is instructive to discuss the different types of phonon scattering mechanisms. Phonons naturally divide themselves into two distinctive types, optical phonons and acoustic phonons. Optical phonons are the phonons which cause the atoms of the unit cell to vibrate in opposite directions. For acoustic phonons, however, the atoms vibrate together, but the wavelength of the vibrations occurs over many unit cells. Typically, the energy of the optical phonons is greater than that of the acoustic phonons. For each type of phonon, two types of interaction occur with the electrons. First, the deformations in the lattice, which arise from the interaction of the lattice with the phonons, changes the energy levels of the electrons, causing transitions to occur. This type of interaction is referred to as non-polar optical phonon scattering for the case of optical phonons and acoustic deformation potential scattering for the case of acoustic phonons.

In polar semiconductors, such as GaN and InN, the deformations which arise also induce localized electric fields. These electric fields also interact with the electrons, causing them to scatter. For the case of optical phonons, the interaction of the electrons with these localized electric fields is referred to as polar optical phonon scattering. For acoustic phonons, however, 
this mechanism is referred to as piezoelectric scattering. Owing to the extremely polar nature of the bonds within $\mathrm{GaN}$ and $\mathrm{InN}$, it turns out that polar optical phonon scattering is very important for these materials. It will be shown that this mechanism alone determines many of the key properties of the electron transport within the III-V nitride semiconductors, GaN and InN.

When the energy of an electron within a valley increases beyond the energy minima of the other valleys, it is also possible for the electrons to scatter from one valley to another. This type of scattering is referred to as inter-valley scattering. It is an important scattering mechanism for many compound semiconductors, and is known to be particularly important for the cases of the wurtzite and zinc-blende phases of $\mathrm{GaN}$ and wurtzite $\mathrm{InN}$; as will be seen later, inter-valley transitions are not particularly important for the case of zinc-blende InN, the large non-parabolicity associated with the lowest energy conduction band valley of this material coupled with its wide conduction band inter-valley energy separation inhibiting the occurrence of inter-valley transitions. Inter-valley scattering is believed to be responsible for the negative differential mobility observed in the velocity-field characteristics associated with many compound semiconductors of interest, such as the wurzite and zinc-blende phases of $\mathrm{GaN}$ and wurtzite $\mathrm{InN}$. For the specific case of zinc-blende InN, however, the large non-parabolicity of the lowest energy conduction band valley leads to a dramatic increase in the electron effective mass for applied electric field strengths approaching the peak field strength, and this leads to the observed negative differential mobility, i.e., the electrons become heavier, and thus slower, within the lowest energy conduction band valley itself.

A derivation of all of these scattering rates, as a function of the semiconductor parameters, can be found in the literature; see, for example, [96,106,111]. A formalism, which closely matches the form used in our ensemble semi-classical three-valley Monte Carlo simulations of electron transport, is found in Nag [95]. Many of the scattering rates that are employed for the purposes of the Monte Carlo simulations of the electron transport within the wurtzite and zinc-blende phases of $\mathrm{GaN}$ and InN, are also explicitly tabulated in Appendix 22 of Shur [96]. Further discussion on 
the Monte Carlo electron transport simulation algorithm is amply provided in the scientific literature [95-97,109,112-115].

\subsection{Parameter selections for the wurtzite and zinc-blende phases of GaN and InN}

\subsubsection{Material and band structural parameter selections}

The material parameter selections, used for the simulations of the electron transport within the wurtzite and zinc-blende phases of $\mathrm{GaN}$ and $\operatorname{InN}$, are as specified in Table $2.1[100,116$ $122]^{9,10,11,12}$. The material parameter selections, corresponding to wurtzite GaN, zinc-blende GaN, wurtzite InN, and zinc-blende InN, are drawn from O'Leary et al. [115], Siddiqua et al. [89], Siddiqua et al. [121], and Siddiqua et al. [121], respectively. The band structural parameter selections, used for the Monte Carlo simulations of the electron transport within the wurtzite and zinc-blende phases of $\mathrm{GaN}$ and $\mathrm{InN}$, are as specified in Table 2.2. These band structural parameter selections, corresponding to wurtzite GaN, zinc-blende GaN, wurtzite InN, and zincblende InN, are drawn from O'Leary et al. [115], Siddiqua et al. [89], Siddiqua et al. [120], and Si-

9 The longitudinal and transverse sound velocities are equal to $\sqrt{\frac{c_{l}}{\rho}}$ and $\sqrt{\frac{c_{t}}{\rho}}$, respectively, where $C_{l}$ and $C_{t}$ denote the respective elastic constants and $\rho$ represents the mass density.

10 Piezoelectric scattering is treated using the well-established zinc-blende scattering rates, and thus, for the cases of the wurtzite crystal structure, a suitably transformed piezoelectric constant, $e_{14}$, must be selected. This may be achieved through the transformation suggested by Bykhovski et al. $[119,120]$. The $e_{14}$ value selected for wurtzite GaN is that suggested by Chin et al. [100]. The $e_{14}$ values selected for the other materials considered in this analysis are set to that corresponding to wurtzite GaN.

${ }^{11}$ All inter-valley deformation potentials are set to $10^{9} \mathrm{eV} / \mathrm{cm}$, following the approach of Gelmont et al. [117].

12 We follow the approach of Bhapkar and Shur [122], and set the inter-valley phonon energies equal to the optical phonon energy, a relationship which holds approximately for the case of GaAs [116]. 
ddiqua et al. [121] respectively; ${ }^{13}$ these band structural parameter selections have been informed by the values suggested by Lambrecht and Segall [123]. The material and band structural parameters, corresponding to the zinc-blende phase of GaAs, are primarily drawn from Littlejohn et al. [116] and Blakemore [124].

\subsubsection{A critical comparison with other compound semiconductors}

It is instructive to contrast the material parameters employed for the simulations of the electron transport within the wurtzite and zinc-blende phases of $\mathrm{GaN}$ and $\operatorname{InN}$ with those corresponding to other compound semiconductors of interest. The particular material parameters which I focus upon for the purposes of this critical comparative analysis are the electron effective mass, the polar optical phonon energy, the static dielectric constant, and the high-frequency dielectric constant, these parameters being known to play important roles in shaping the nature of the electron transport. The dependence of these parameters on the $E_{0}$ energy gap at $300 \mathrm{~K}$, as defined by Adachi [125], will be the focus of this analysis. ${ }^{14}$ The other compound semiconductors considered in this analysis are aluminum arsenide (AIAs), aluminum phosphide (AIP), the zinc-blende phase of cadmium selenide (zb-CdSe), the wurtzite phase of cadmium sulphide (w-CdS), the zinc-blende phase of cadmium sulphide (zb-CdS), gallium antimonide (GaSb), gallium phosphide (GaP), indium antimonide (InSb), indium arsenide (InAs), indium pho-

\footnotetext{
${ }^{13}$ Each conduction band band structure is modeled as possessing three distinct "valleys", each of the valley minima (with respect to energy) corresponding to a minima in the corresponding actual conduction band structure. The valley locations are specified according to the locations of the local energy minima in the band structures, the degeneracy of each valley, the effective mass of the electrons at each valley minimum, and the non-parabolicity coefficient corresponding to each valley also being specified.

${ }^{14}$ For the case of direct-gap semiconductors, the $E_{0}$ energy gap coincides with the regular energy gap, $E_{g}$. For the case of indirect-gap semiconductors, however, the $E_{0}$ energy gap exceeds $E_{g}$. Adachi [125] refers to the $E_{0}$ energy gap as the lowest direct-gap energy gap.
} 
sphide (InP), zinc selenide ( $\mathrm{ZnSe})$, and zinc telluride (ZnTe). While the energy gaps associated with the wurtzite and zinc-blende phases of $\mathrm{GaN}$ and $\mathrm{InN}$ are direct, some of the other compound semiconductors considered in this critical comparative analysis possess indirect energy gaps, wherein the actual energy gap, $E_{g}$, i.e., the difference in energy between the minimum energy of the conduction band and the maximum energy of the valence band, does not coincide with the $E_{0}$ energy gap. The material and band structural parameters, used for this critical comparative analysis, are drawn from Adachi [125] and Sze and $\mathrm{Ng}$ [126]. The material and band structural parameters, corresponding to these other compound semiconductors, are tabulated in Table 2.3 [127]. A bar chart of the energy gaps, $E_{g}$, at $300 \mathrm{~K}$, corresponding to a representative sampling of elemental and compound semiconductors, including some of the other compound semiconductors considered in this critical comparative analysis, is depicted in Figure 2.3.

In Figure 2.4, I plot the electron effective mass, expressed in units of the free electron mass, $\mathrm{m}_{\mathrm{e}}$, as a function of the $300 \mathrm{~K} E_{0}$ energy gap corresponding to the materials considered in this critical comparative analysis. It is noted that the electron effective mass monotonically increases in response to increases in the $E_{0}$ energy gap. The results of a linear least-squares fit, depicted with the dashed line in Figure 2.4, suggests that the electron effective mass essentially scales linearly with the $E_{0}$ energy gap. Indeed, the deviations about this linear least-squares fit are noted to be relatively minor. The electron effective mass plays an important role in defining the low-field electron drift mobility, the higher this mass the lower the corresponding low-field electron drift mobility. This result suggests that the low-field electron drift mobility associated with a semiconductor will diminish as the $E_{0}$ energy gap is increased.

In Figure 2.5, I plot the polar optical phonon energy, $\hbar \omega_{0}$, as a function of the $300 \mathrm{~K} E_{0}$ energy gap corresponding to the materials considered in this critical comparative analysis. I generally find that the polar optical phonon energy monotonically increases in response to increases in the $E_{0}$ energy gap. In this case, however, there is a considerable amount of scatter about the trend; this scatter is sufficiently great that a linear least-squares fit, while indicative of 
Table 2.3 The material and band structural parameters, corresponding to the other common compound semiconductors considered in this analysis. This table has been modified from @ Siddiqua, P. \& O'Leary, S.K. (2018). Electron transport within the wurtzite and zinc-blende phases of gallium nitride and indium nitride, Journal of Materials Science, 29, 3511-3567. Page 3524. Adapted with permission from publisher. The material and band structural parameters, used for this critical comparative analysis, are drawn from Adachi [124] and Sze and $\mathrm{Ng}[125]$

\begin{tabular}{|c|c|c|c|c|c|c|c|c|c|c|}
\hline Semi. & Type & Crys. & B. & $\overline{E_{g}(e V)}$ & $\mathrm{E}_{0}(\mathrm{eV})$ & $\mathrm{m}^{*}\left(\mathrm{~m}_{\mathrm{e}}\right)$ & $\hbar \omega_{0}(\mathrm{meV})$ & $\epsilon_{\mathrm{s}}$ & $\epsilon_{\infty}$ & $\mathrm{E}_{\mathrm{br}}(\mathrm{V} / \mathrm{cm})$ \\
\hline $\operatorname{lnSb}$ & III-V & $\mathrm{zb}$ & $\mathrm{D}$ & 0.17 & 0.17 & 0.013 & 23.65 & 17.2 & 15.3 & $10^{3}$ \\
\hline $\ln A s$ & III-V & $z b$ & $\mathrm{D}$ & 0.359 & 0.359 & 0.024 & 29.93 & 14.3 & 11.6 & $4 \times 10^{4}$ \\
\hline$w-\operatorname{In} N$ & III-V & w & $\bar{D}$ & 0.70 & 0.70 & 0.04 & 73.0 & 15.3 & 8.4 & $10^{6}$ \\
\hline$z b-\operatorname{In} N$ & III-V & w & $\mathrm{D}$ & 0.70 & 0.70 & 0.04 & 73.0 & 15.3 & 8.4 & $10^{6}$ \\
\hline GaSb & III-V & $\mathrm{zb}$ & $\bar{D}$ & 0.72 & 0.72 & 0.039 & 28.9 & 15.5 & 14.2 & $5 \times 10^{4}$ \\
\hline $\ln P$ & III-V & $z b$ & $\mathrm{D}$ & 1.35 & 1.35 & 0.07927 & 42.95 & 12.9 & 9.9 & $4.5 \times 10^{5}$ \\
\hline GaAs & III-V & $z b$ & D & 1.43 & 1.43 & 0.067 & 35.3 & 12.9 & 10.86 & $4 \times 10^{5}$ \\
\hline AlSb & III-V & $z b$ & ID & 1.615 & 2.27 & 0.14 & 42.16 & 11.21 & 9.88 & $5.8 \times 10^{5}$ \\
\hline zb-CdSe & II-VI & $\mathrm{zb}$ & $\mathrm{D}$ & 1.675 & 1.675 & 0.119 & 26.2 & 9.6 & 6.2 & $6.3 \times 10^{5}$ \\
\hline AlAs & III-V & $\mathrm{zb}$ & ID & 2.15 & 3.01 & 0.124 & 49.8 & 10.06 & 8.16 & $1.2 \times 10^{6}$ \\
\hline $\mathrm{GaP}$ & III-V & $z b$ & ID & 2.261 & 2.76 & 0.114 & 49.91 & 11.0 & 8.8 & $1.15 \times 10^{6}$ \\
\hline ZnTe & $\mathrm{II}-\mathrm{VI}$ & $\mathrm{zb}$ & $\mathrm{D}$ & 2.27 & 2.27 & 0.117 & 26.0 & 9.4 & 6.9 & $1.3 \times 10^{6}$ \\
\hline$z b-C d S$ & $\mathrm{II}-\mathrm{VI}$ & $z b$ & $\bar{D}$ & 2.46 & 2.46 & 0.14 & 37.6 & 9.8 & 5.4 & $1.6 \times 10^{6}$ \\
\hline AIP & III-V & $z b$ & ID & 2.48 & 3.91 & 0.220 & 62.12 & 9.6 & 7.4 & $1.6 \times 10^{6}$ \\
\hline$w-C d S$ & II-VI & w & $\mathrm{D}$ & 2.501 & 2.501 & 0.151 & 37.7 & 9.6 & 5.35 & $1.7 \times 10^{6}$ \\
\hline ZnSe & $\mathrm{II}-\mathrm{VI}$ & $z b$ & $\mathrm{D}$ & 2.721 & 2.721 & 0.137 & 31.2 & 8.9 & 5.9 & $2.0 \times 10^{6}$ \\
\hline zb-GaN & III-V & $\mathrm{zb}$ & $\mathrm{D}$ & 3.20 & 3.20 & 0.15 & 91.2 & 8.9 & 5.35 & $3.1 \times 10^{6}$ \\
\hline w-GaN & III-V & $\mathrm{W}$ & $\mathrm{D}$ & 3.39 & 3.39 & $0 . .20$ & 91.2 & 8.9 & 5.35 & $2.95 \times 10^{6}$ \\
\hline $\mathrm{ZnO}$ & $\mathrm{II}-\mathrm{VI}$ & w & $\mathrm{D}$ & 3.40 & 3.40 & 0.17 & 72.0 & 8.2 & 3.7 & $3.5 \times 10^{6}$ \\
\hline
\end{tabular}




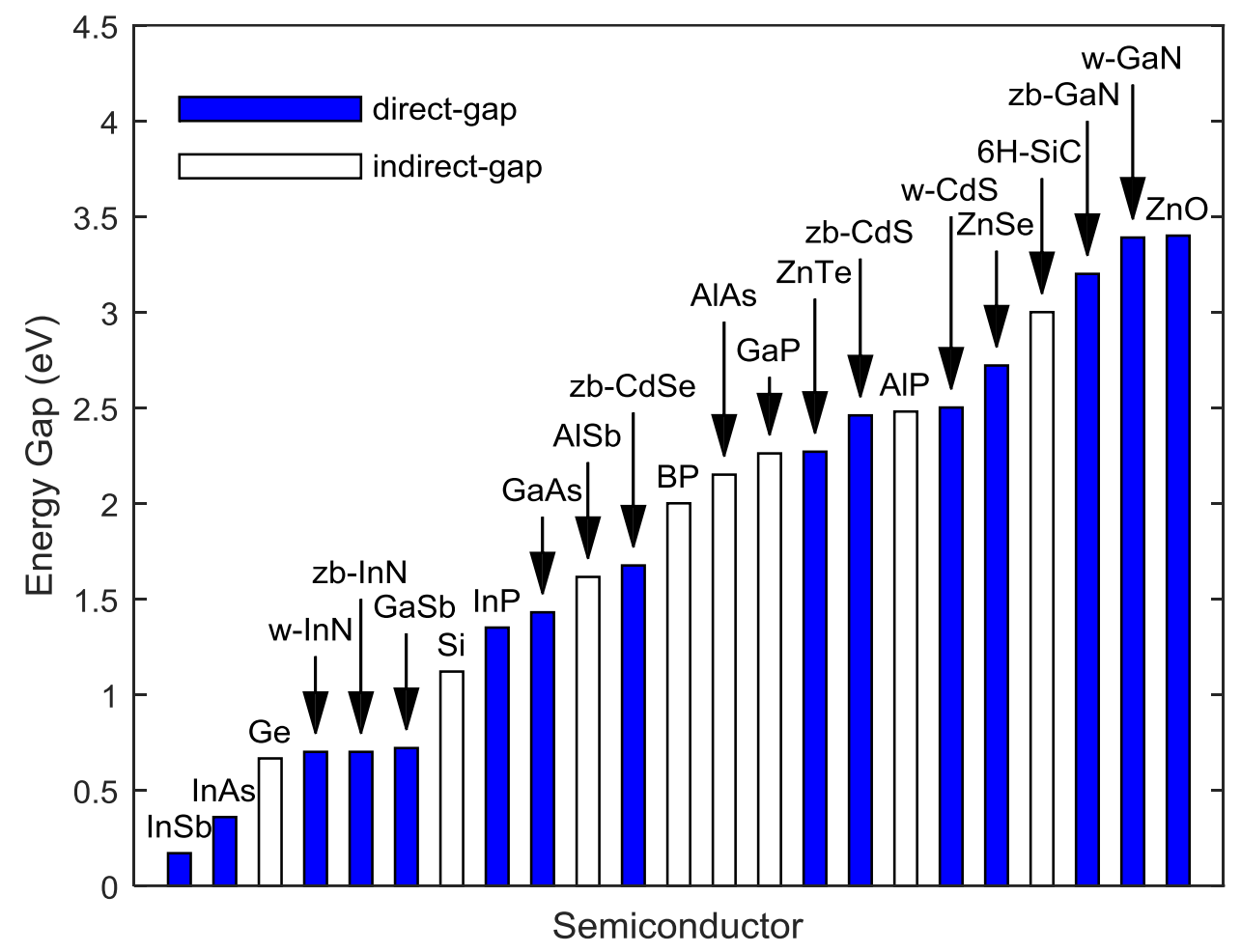

Figure 2.3: The energy gap, $E_{g}$, at $300 \mathrm{~K}$, for a number of elemental and compound semiconductors. This figure is modified from ( ) Siddiqua, P., \& O'Leary, S. (2018). Electron transport within the wurtzite and zincblende phases of gallium nitride and indium nitride. Journal of Materials Science, 29, 3511-3567. Page 3523. Adapted with permission from publisher. The online version of this figure is depicted in color. 


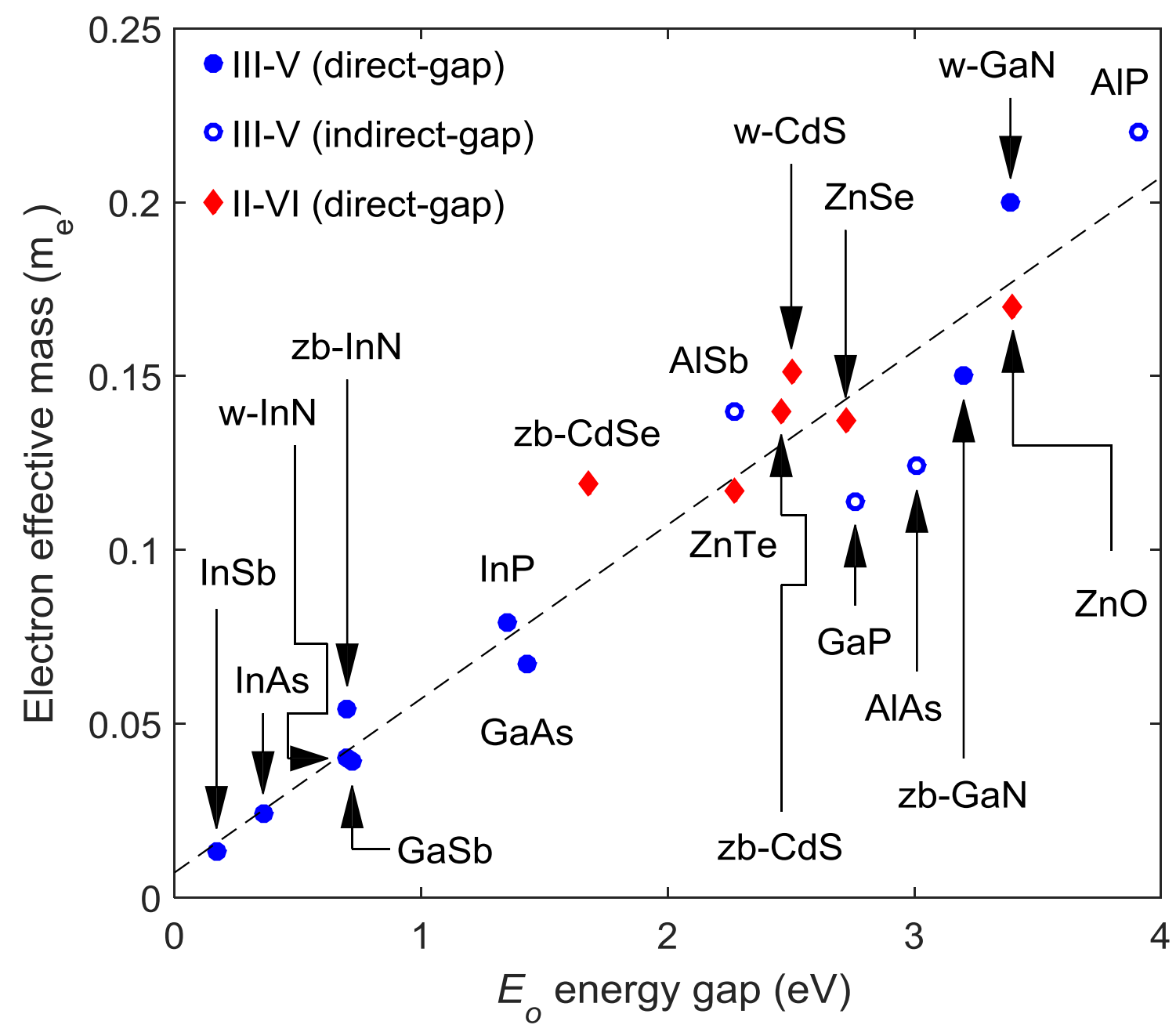

Figure 2.4: The electron effective mass associated with the lowest energy valley as a function of the $E_{0}$ energy gap, at $300 \mathrm{~K}$, for the semiconductors considered in this analysis and other compound semiconductors. The data for this plot is drawn from Table 2.3. This figure is modified from (C) Siddiqua, P., \& O'Leary, S. (2018). Electron transport within the wurtzite and zinc-blende phases of gallium nitride and indium nitride. Journal of Materials Science, 29, 3511-3567. Page 3524. Adapted with permission from publisher. The online version of this figure is depicted in color. 


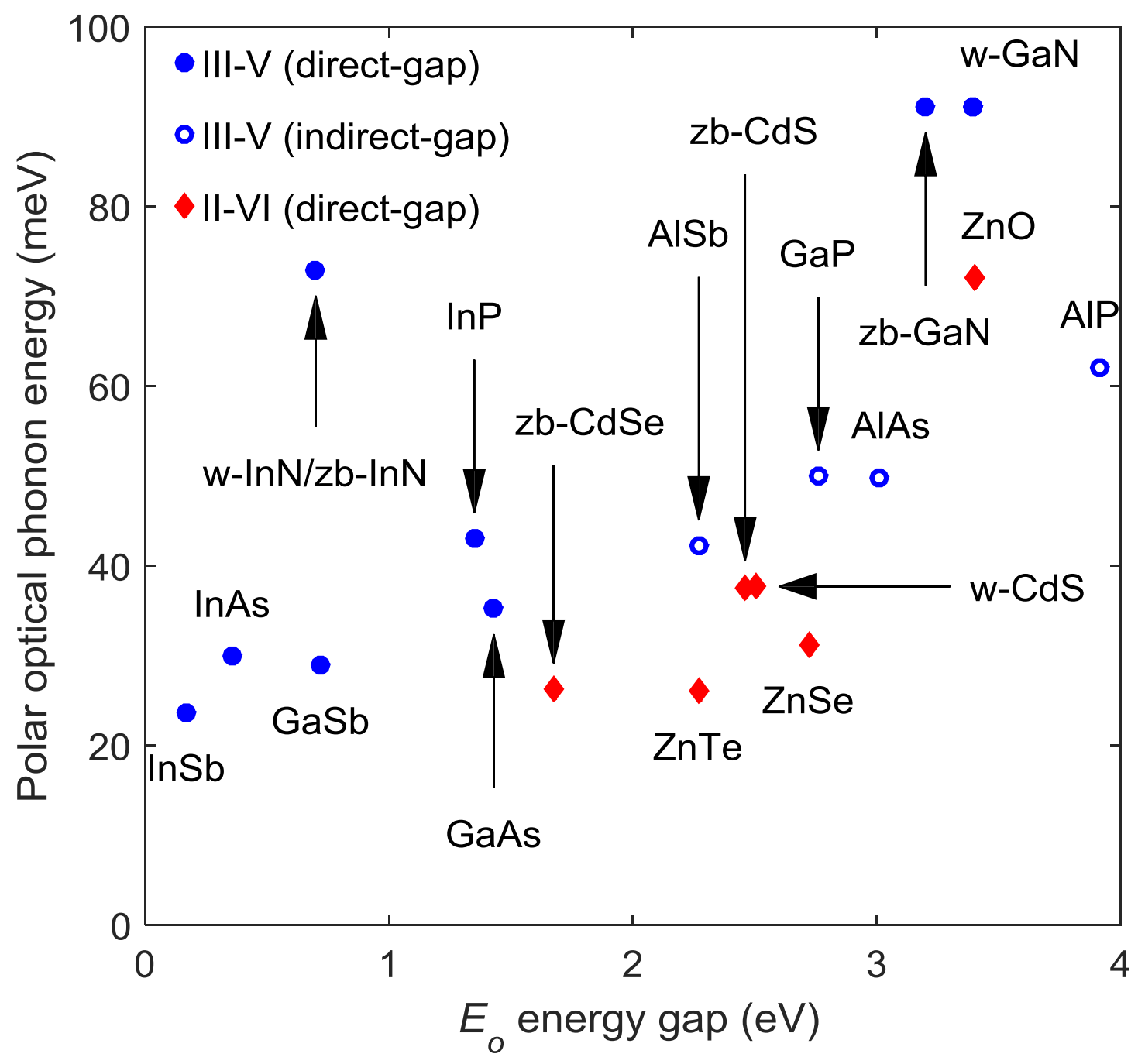

Figure 2.5: The polar optical phonon energy as a function of the $E_{0}$ energy gap, at $300 \mathrm{~K}$, for the semiconductors considered in this analysis and other compound semiconductors. The data for this plot is drawn from Table 2.3. This figure is modified from @ ( Siddiqua, P., \& O'Leary, S. (2018). Electron transport within the wurtzite and zinc-blende phases of gallium nitride and indium nitride. Journal of Materials Science, 29, 3511-3567. Page 3524. Adapted with permission from publisher. The online version of this figure is depicted in color. 
a general trend, is not statistically significant. The polar optical phonon energy plays a critical role in determining the nature of the high-field electron transport. In particular, it is known that the highfield saturation electron drift velocity is, in large measure, determined by it [1]. This result suggests that semiconductors with wider $E_{0}$ energy gaps favor higher saturation electron drift velocities [1].

In Figures 2.6 and 2.7, I plot the relative static and relative high-frequency dielectric constants as a function of the $300 \mathrm{~K} E_{0}$ energy gap corresponding to the materials considered in this critical comparative analysis. It can be noted that these dielectric constants diminish as the $E_{0}$ energy gap increases. It may be noted, however, that the scatter found in these cases is even greater than that found for the case of the polar optical phonon energy. Diminished dielectric constants favor enhanced device performance, pointing once again to an advantage offered by the wider energy gap semiconductors.

Finally, it is instructive to plot the breakdown electric field strength as a function of the 300 $\mathrm{K} E_{0}$ energy gap corresponding to the materials considered in this critical comparative analysis. While the breakdown field strength is not an independent material parameter itself, as were the other parameters considered in this critical comparative analysis (in fact, the breakdown field strength is determinable from the fundamental material properties), it is useful to be aware of it, as its value defines the range of electric field strengths that may be applied to a given semiconductor under investigation. A plot of the breakdown field strength as a function of the 300 $\mathrm{K} E_{0}$ energy gap corresponding to the materials considered in this critical comparative analysis is depicted in Figure 2.8. We note that generally the breakdown field strength increases as the 300 $\mathrm{K} E_{0}$ energy gap increases. This suggests that the wider energy gap materials can better serve in applications demanding higher electric field strengths, such as in high-power device structures. 


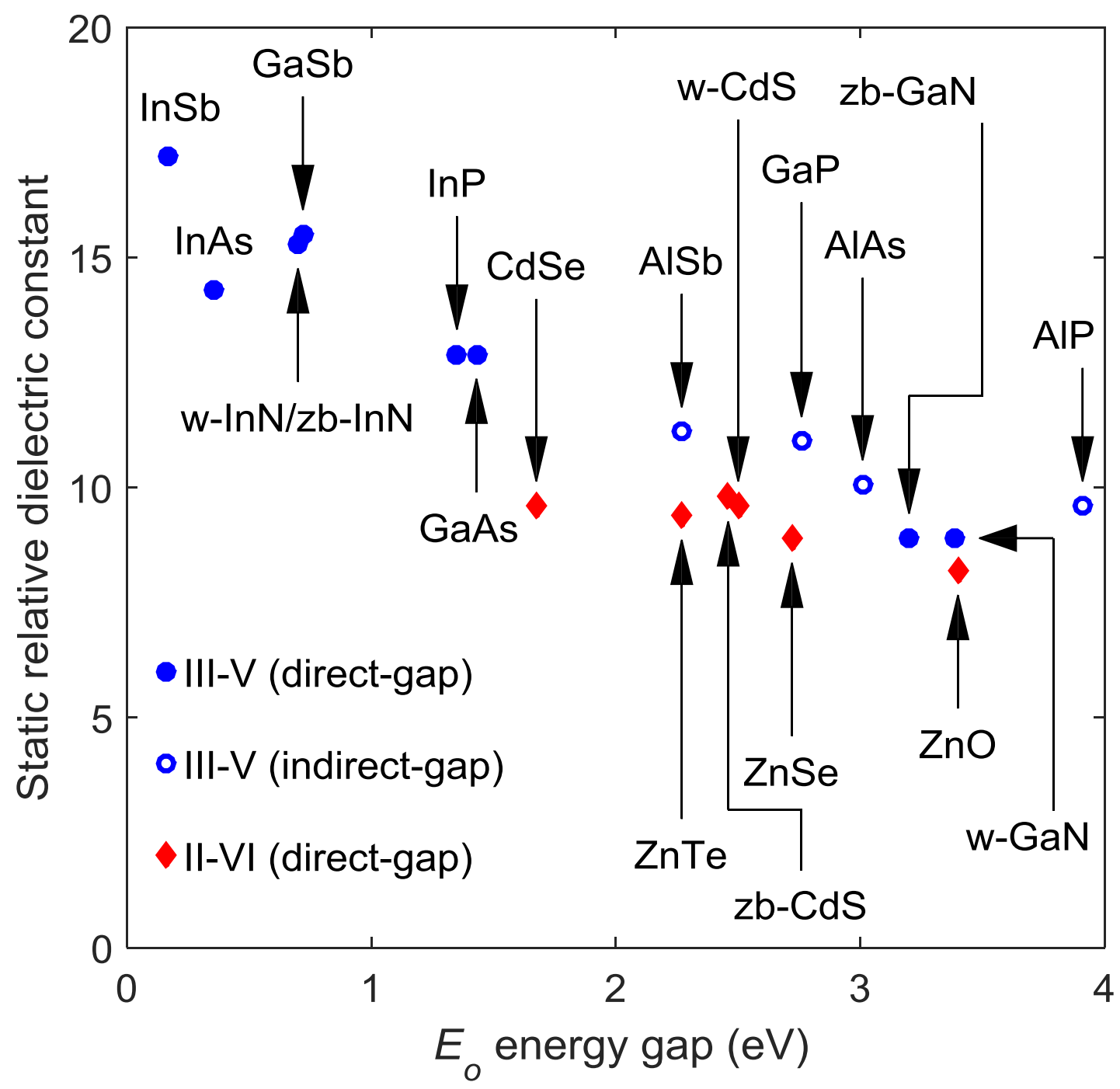

Figure 2.6: The static relative dielectric constant as a function of the $E_{0}$ energy gap, at $300 \mathrm{~K}$, for the semiconductors considered in this analysis and other compound semiconductors. The data for this plot is drawn from Table 2.3. This figure is modified from @ S Siddiqua, P., \& O'Leary, S. (2018). Electron transport within the wurtzite and zinc-blende phases of gallium nitride and indium nitride. Journal of Materials Science, 29, 3511-3567. Page 3525. Adapted with permission from publisher. The online version of this figure is depicted in color. 


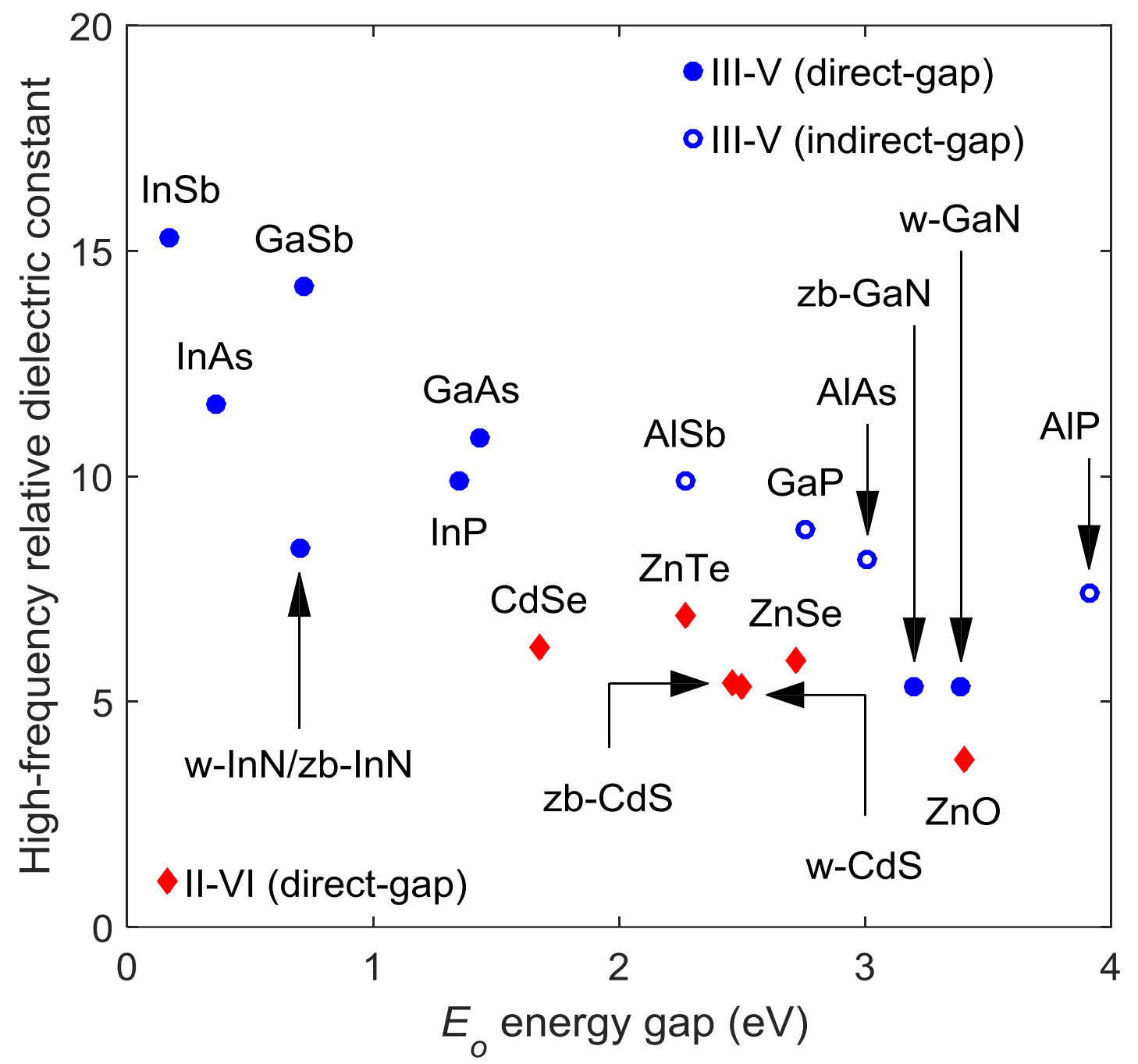

Figure 2.7: The high-frequency relative dielectric constant as a function of the $E_{0}$ energy gap, at $300 \mathrm{~K}$, for the semiconductors considered in this analysis and other compound semiconductors. The data for this plot is drawn from Table 2.3. This figure is modified from ( ) Siddiqua, P., \& O'Leary, S. (2018). Electron transport within the wurtzite and zinc-blende phases of gallium nitride and indium nitride. Journal of Materials Science, 29, 3511-3567. Page 3525. Adapted with permission from publisher. The online version of this figure is depicted in color. 


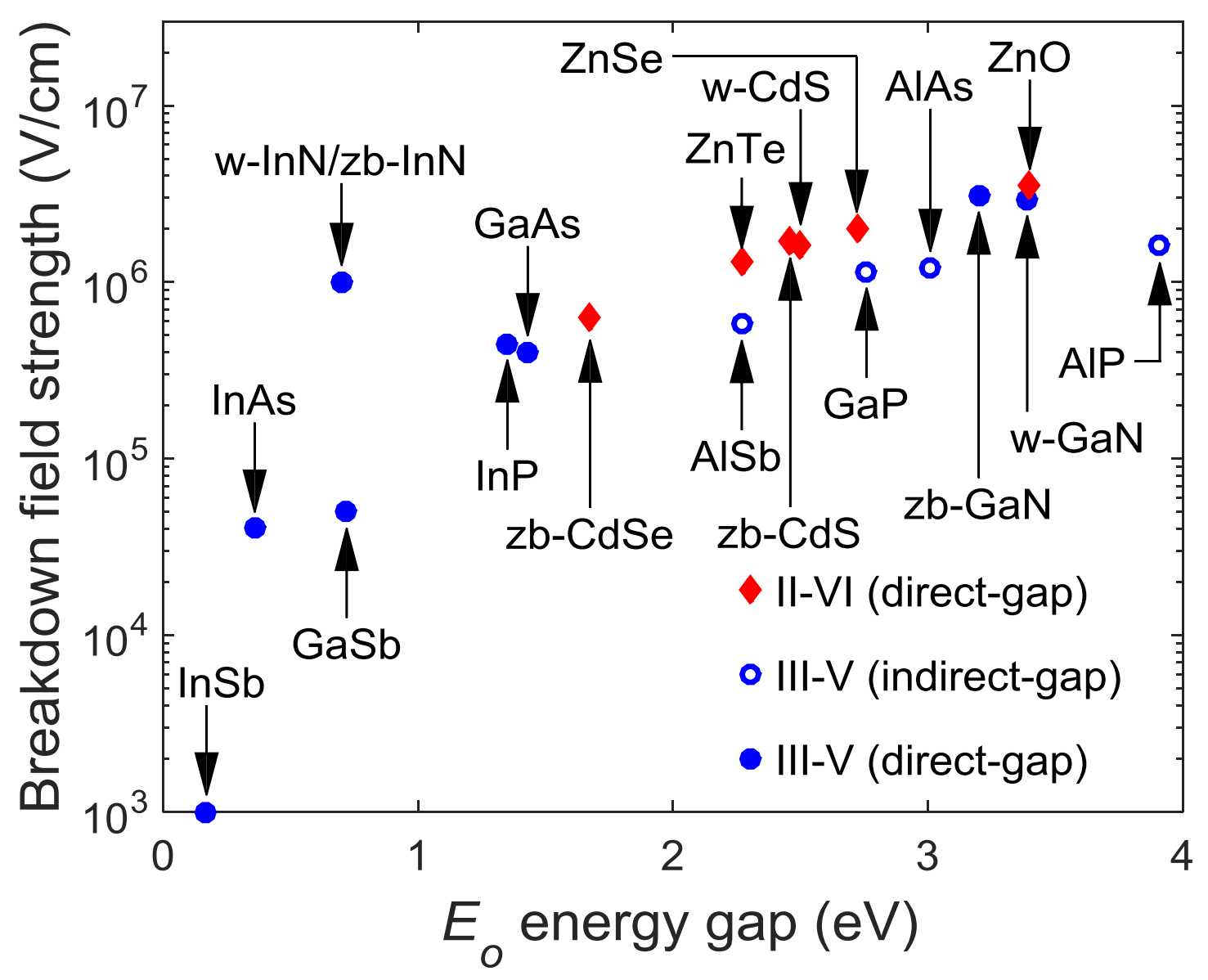

Figure 2.8: The breakdown field strength as a function of the $E_{0}$ energy gap, at $300 \mathrm{~K}$, for the semiconductors considered in this analysis and other compound semiconductors. The data for this plot is drawn from Table 2.3. This figure is modified from (c) Siddiqua, P., \& O'Leary, S. (2018). Electron transport within the wurtzite and zinc-blende phases of gallium nitride and indium nitride. Journal of Materials Science, 29, 3511-3567. Page 3525. Adapted with permission from publisher. The online version of this figure is depicted in color. 


\subsection{The Monte Carlo simulation approach}

For the purposes of this analysis of the electron transport within the wurtzite and zincblende phases of $\mathrm{GaN}$ and $\mathrm{InN}$, I employ ensemble semi-classical three-valley Monte Carlo electron transport simulations. The scattering mechanisms considered are: (1) ionized impurity, (2) polar optical phonon, (3) piezoelectric, and (4) acoustic deformation potential. Inter-valley scattering is also considered. We assume that all donors are ionized and that the free electron concentration is equal to the dopant concentration. For the steady-state electron transport simulations, the motion of three-thousand electrons is examined, while for the transient electron transport simulations, the motion of ten-thousand electrons is considered. For the simulations, the crystal temperature is set to $300 \mathrm{~K}$ and the doping concentration is set to $10^{17} \mathrm{~cm}^{-3}$ for most cases. Electron degeneracy effects are accounted for by means of the rejection technique of Lugli and Ferry [128]. Electron screening is also accounted for following the Brooks-Herring method [129]. Further details of our approach are discussed in the literature $[64,115,117,118,122,130-141]$.

\subsection{Scattering rates in the wurtzite and zinc-blende phases of GaN and InN}

In order to develop an appreciation for the role that the individual scattering mechanisms play in shaping the nature of the electron transport within the different materials under investigation in this analysis, it is instructive to contrast the dependence of the different scattering rates on the magnitude of the electron wave-vector, $\vec{k}$. In Figures 2.9, 2.10, 2.11, and 2.12, I plot the various scattering rates as a function of the magnitude of the electron wave-vector, $\vec{k}$, for the wurtzite and zinc-blende phases of $\mathrm{GaN}$ and $\mathrm{InN}$. These are the scattering rates corresponding to the lowest energy valley in the conduction band, i.e., the $\Gamma$ valley for the compound semiconductors considered in this thesis. The electrons in the upper valleys are found to experience similar scattering rates; each of the scattering rates considered in the simulations of 
the electron transport within the wurtzite and zinc-blende phases of $\mathrm{GaN}$ and $\operatorname{InN}$, is described, in detail, by Nag [95]. For the ionized impurity, polar optical phonon, and piezoelectric scattering mechanisms, screening effects are taken into account. These screening effects tend to lower the scattering rates when the electron concentrations are high. Scattering results, corresponding to the specific case of GaAs, are represented in Figure 2.13, these results being depicted primarily for benchmarking purposes. 


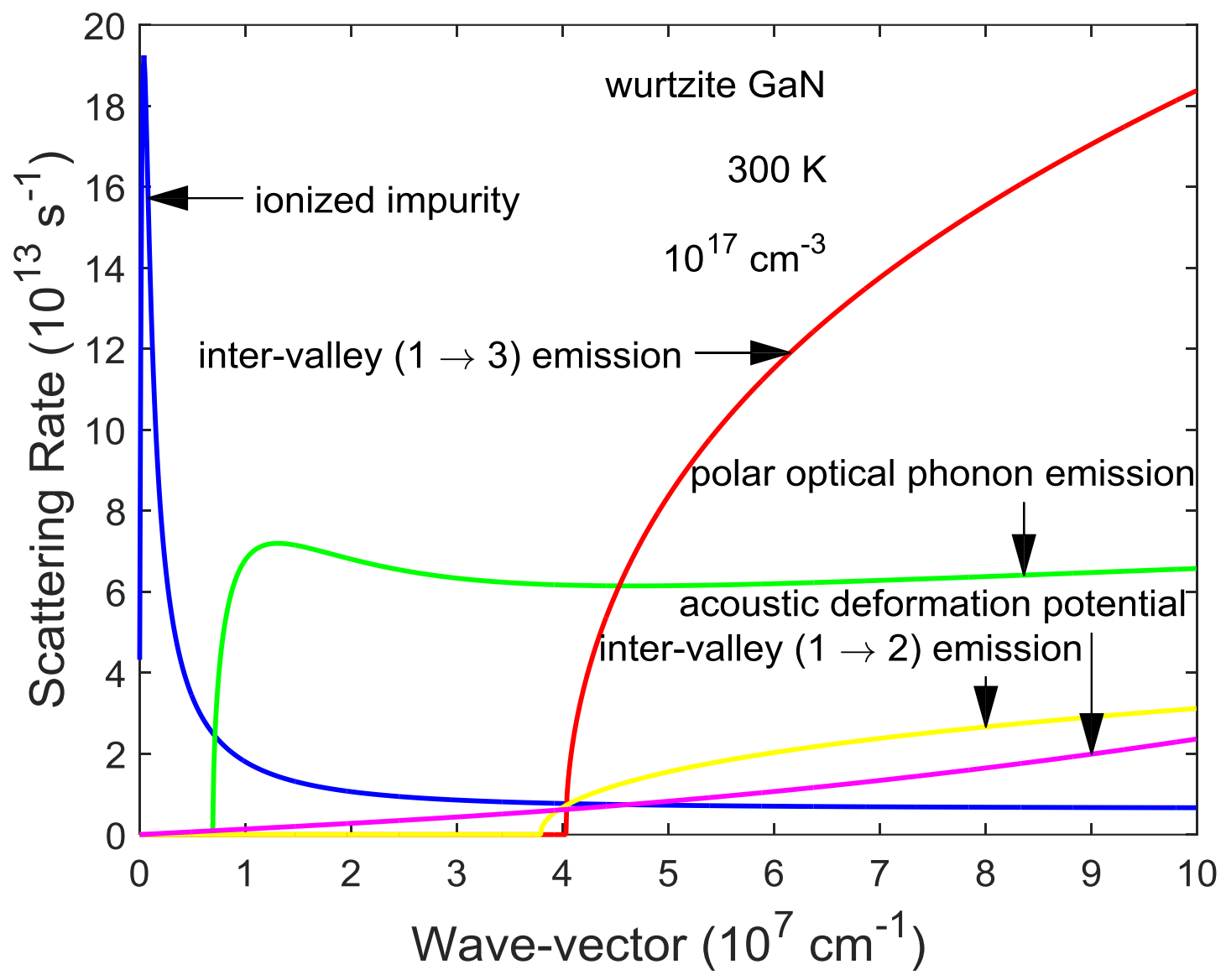

Figure 2.9.a: The scattering rates for the lowest energy $(\ulcorner)$ valley as a function of the magnitude of the wave-vector for bulk wurtzite GaN. The scattering mechanisms considered are: (1) ionized impurity (blue), (2) polar optical phonon emission (green), (3) inter-valley $(1 \rightarrow 3)$ emission (red), (4) inter-valley (1 $\rightarrow 2)$ emission (yellow), (5) acoustic deformation potential (magenta). This figure is modified from (C) Siddiqua, P., \& O'Leary, S. (2018). Electron transport within the wurtzite and zinc-blende phases of gallium nitride and indium nitride. Journal of Materials Science, 29, 3511-3567. Page 3526. Adapted with permission from publisher. The online version of this figure is depicted in color. 


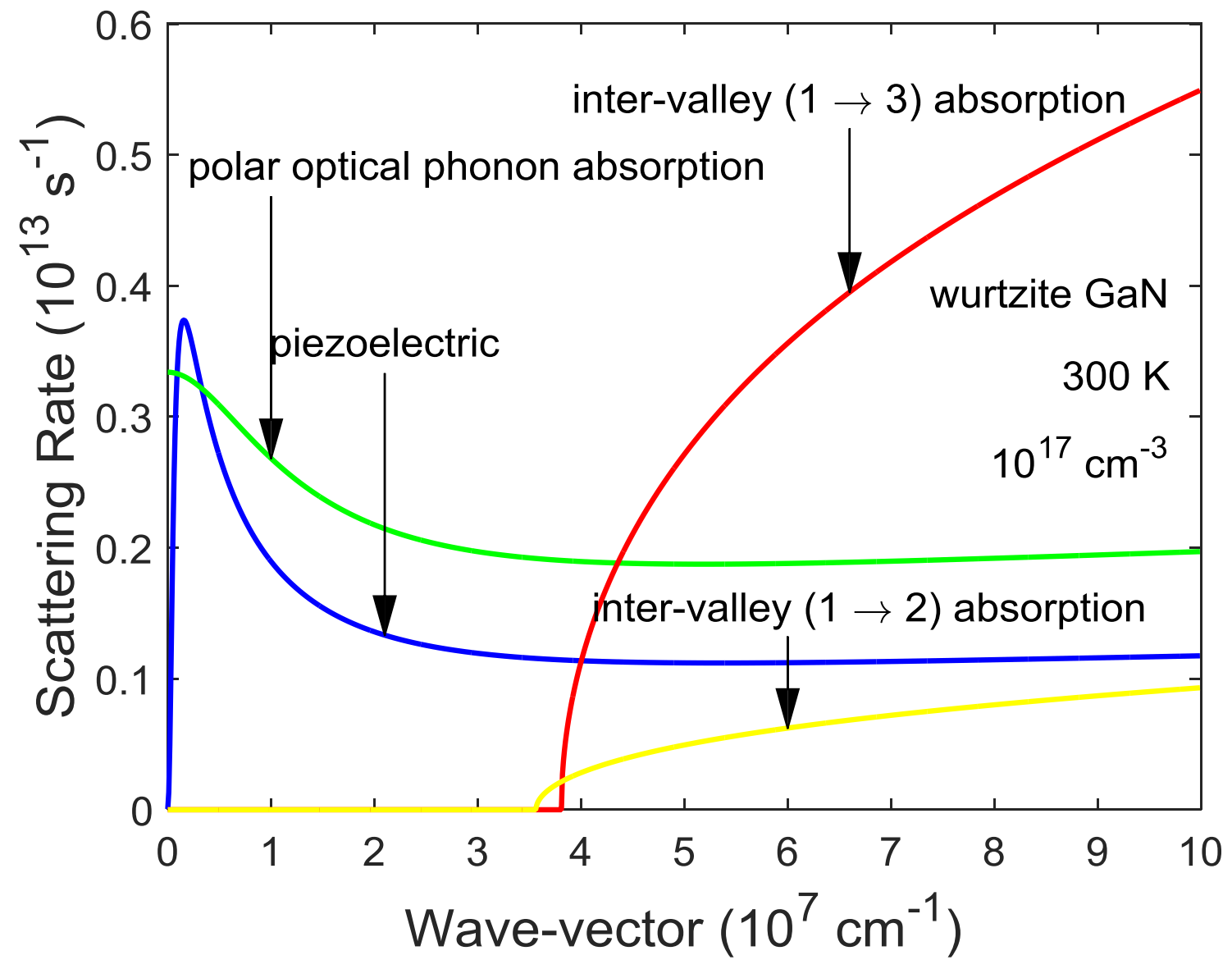

Figure 2.9.b: The scattering rates for the lowest energy $(\Gamma)$ valley as a function of the magnitude of the wave-vector for bulk wurtzite GaN. The scattering mechanisms considered are: (1) piezoelectric (blue), (2) polar optical phonon absorption (green), (3) inter-valley $(1 \rightarrow 3)$ absorption (red), and (4) inter-valley ( $1 \rightarrow$ 2) absorption (yellow). This figure has been modified from @ Siddiqua, P. \& O'Leary, S.K. (2018). Electron transport within the wurtzite and zinc-blende phases of gallium nitride and indium nitride, Journal of Materials Science, 29, 3511-3567. Page 3526. Adapted with permission from publisher. The online version of this figure is depicted in color. 


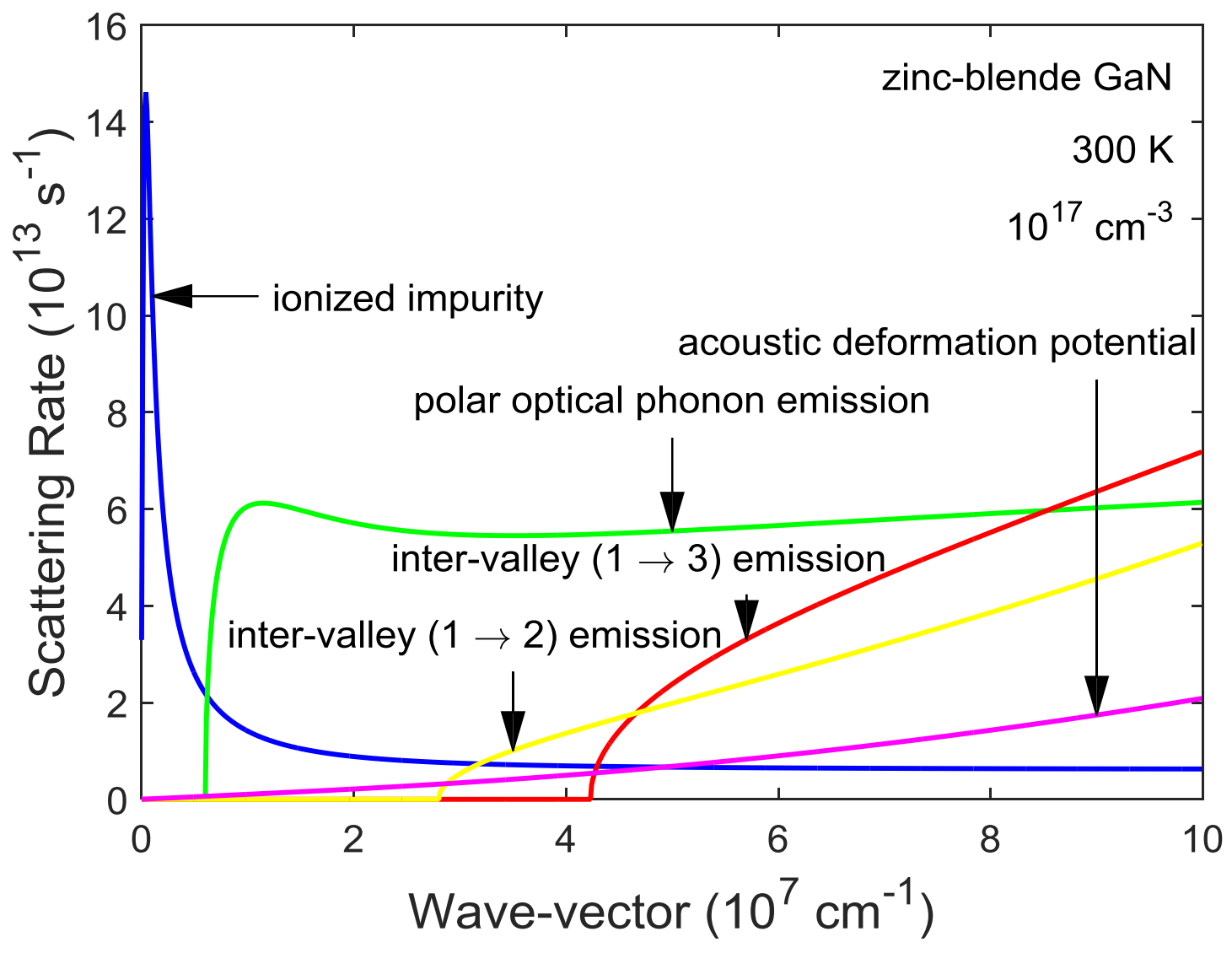

Figure 2.10.a: The scattering rates for the lowest energy $(\Gamma)$ valley as a function of the magnitude of the wave-vector for bulk zinc-blende GaN. The scattering mechanisms considered are: (1) ionized impurity (blue), (2) polar optical phonon emission (green), (3) inter-valley (1 $\rightarrow 3$ ) emission (red), (4) inter-valley ( $1 \rightarrow 2$ ) emission (yellow), (5) acoustic deformation potential (magenta). This figure has been modified from (C) Siddiqua, P. \& O'Leary, S.K. (2018). Electron transport within the wurtzite and zinc-blende phases of gallium nitride and indium nitride, Journal of Materials Science, 29, 3511-3567. Page 3526. Adapted with permission from publisher. The online version of this figure is depicted in color. 


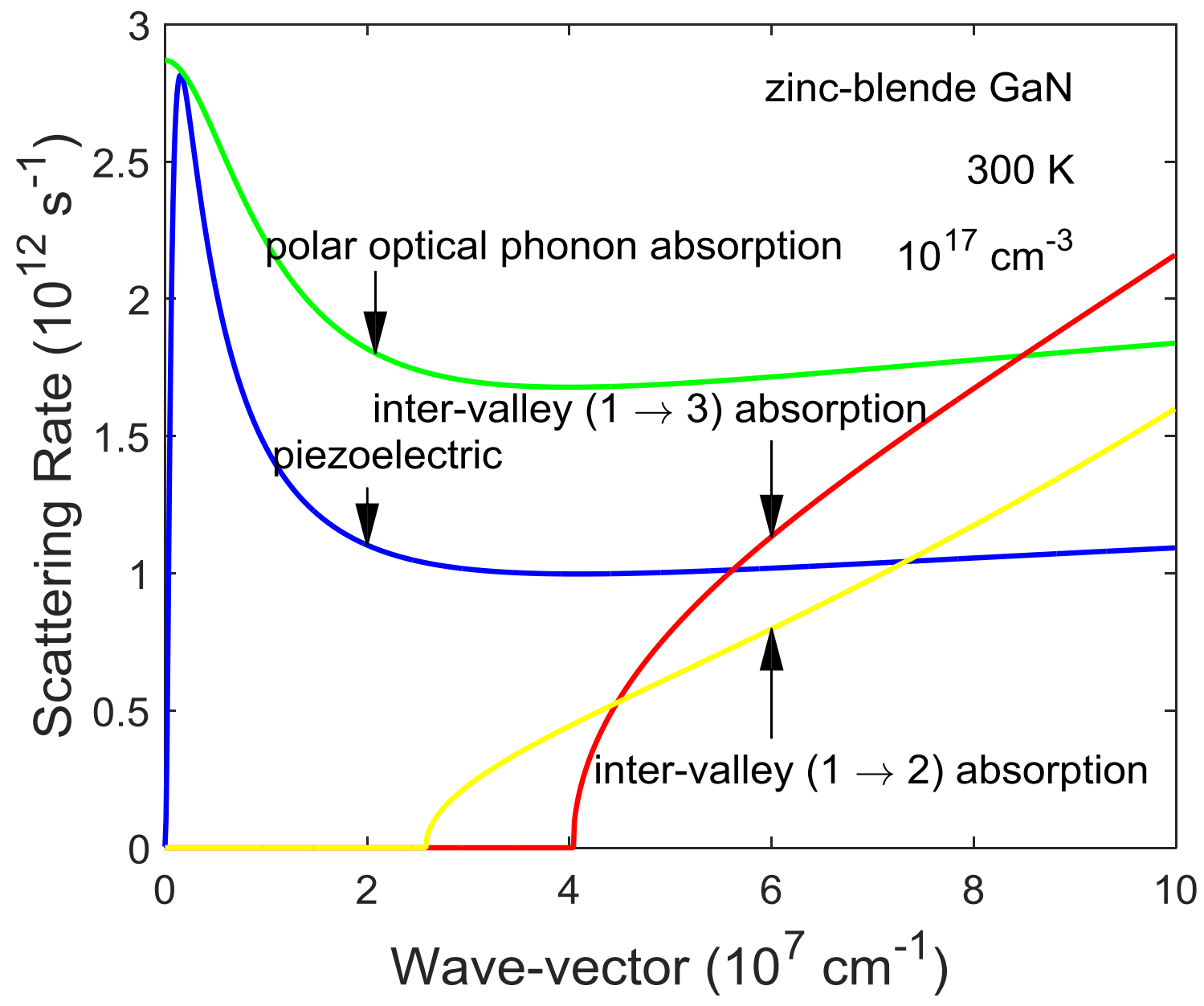

Figure 2.10.b: The scattering rates for the lowest energy $(Г)$ valley as a function of the magnitude of the wave-vector for bulk zinc-blende GaN. The scattering mechanisms considered are :(1) piezoelectric (blue), (2) polar optical phonon absorption (green), (3) inter-valley $(1 \rightarrow 3$ ) absorption (red), and (4) inter-valley $(1 \rightarrow 2$ ) absorption (yellow). This figure has been modified from ( ) Siddiqua, P. \& O'Leary, S.K. (2018). Electron transport within the wurtzite and zinc-blende phases of gallium nitride and indium nitride, Journal of Materials Science, 29, 3511-3567. Page 3526. Adapted with permission from publisher. The online version of this figure is depicted in color. 


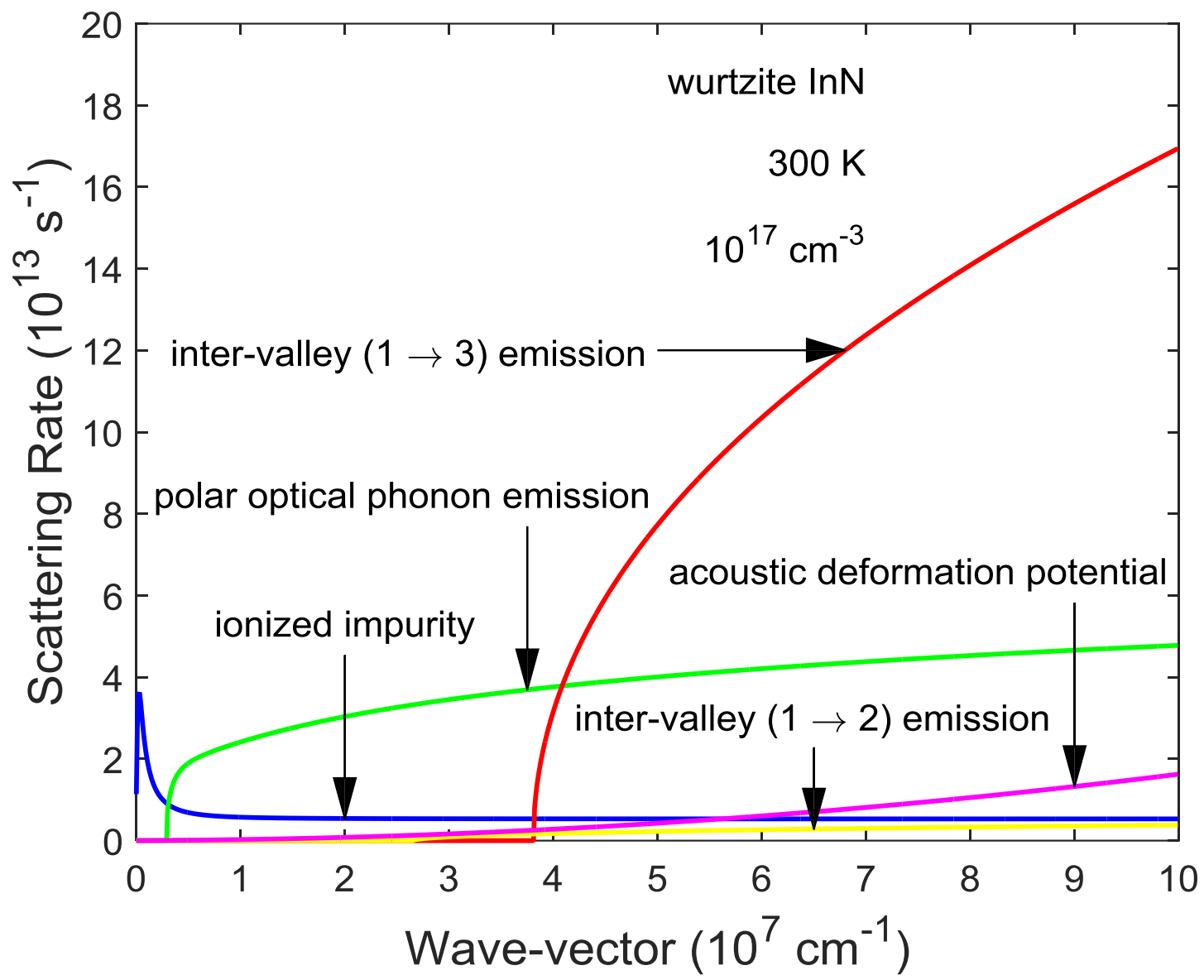

Figure 2.11.a: The scattering rates for the lowest energy $(Г)$ valley as a function of the magnitude of the wave-vector for bulk wurtzite InN. The scattering mechanisms considered are: (1) ionized impurity (blue), (2) polar optical phonon emission (green), (3) inter-valley $(1 \rightarrow 3$ ) emission (red), (4) inter-valley $(1 \rightarrow 2)$ emission (yellow), (5) acoustic deformation potential (magenta). This figure has been modified from (C) Siddiqua, P. \& O'Leary, S.K. (2018). Electron transport within the wurtzite and zinc-blende phases of gallium nitride and indium nitride, Journal of Materials Science, 29, 3511-3567. Page 3527. Adapted with permission from publisher. The online version of this figure is depicted in color. 


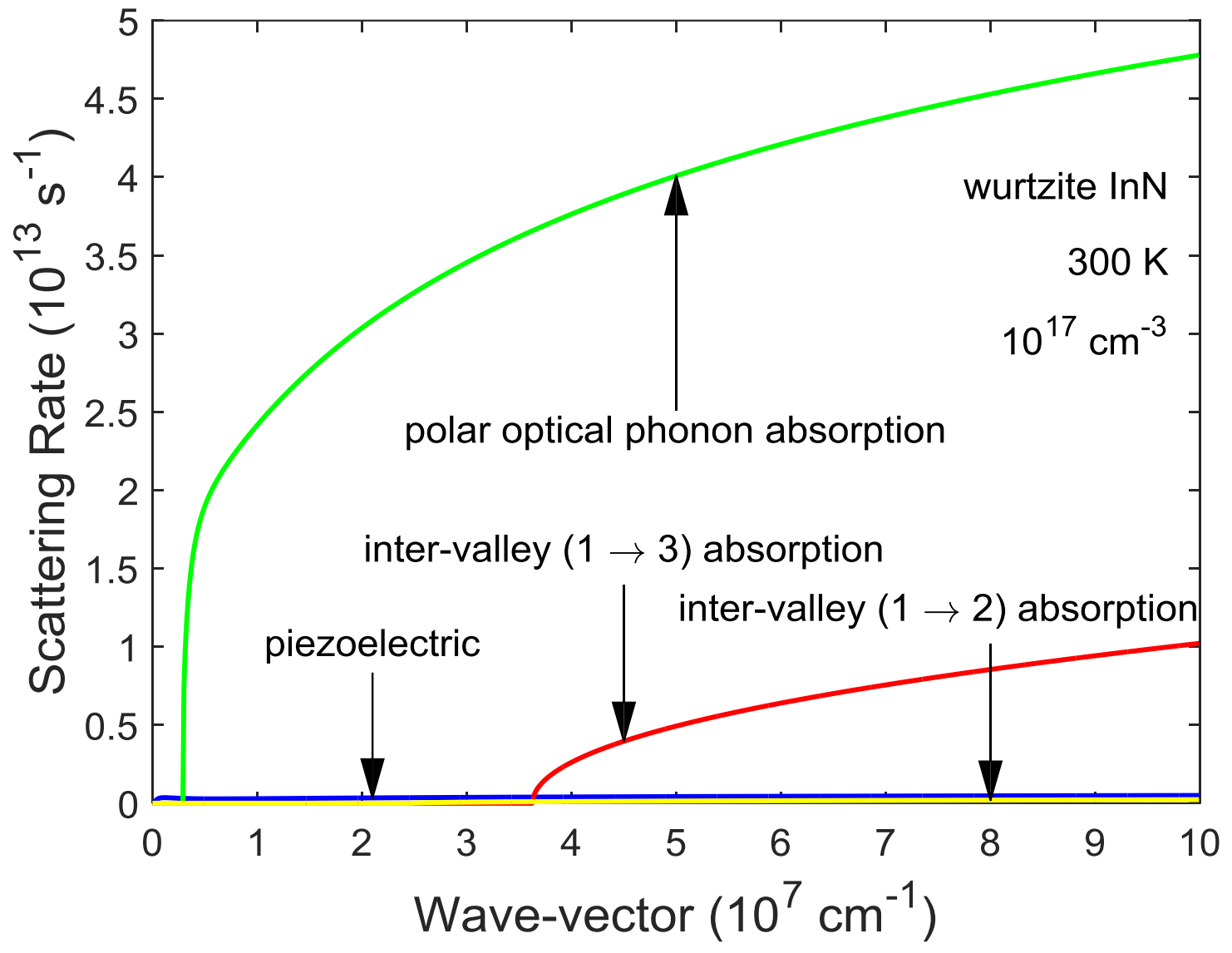

Figure 2.11.b: The scattering rates for the lowest energy $(Г)$ valley as a function of the magnitude of the wave-vector for bulk wurtzite InN. The scattering mechanisms considered are: (1) piezoelectric (blue), (2) polar optical phonon absorption (green), (3) inter-valley $(1 \rightarrow 3)$ absorption (red), and (4) inter-valley $(1 \rightarrow$ 2) absorption (yellow). This figure has been modified from @ Siddiqua, P. \& O'Leary, S.K. (2018). Electron transport within the wurtzite and zinc-blende phases of gallium nitride and indium nitride, Journal of Materials Science, 29, 3511-3567. Page 3527. Adapted with permission from publisher. The online version of this figure is depicted in color. 


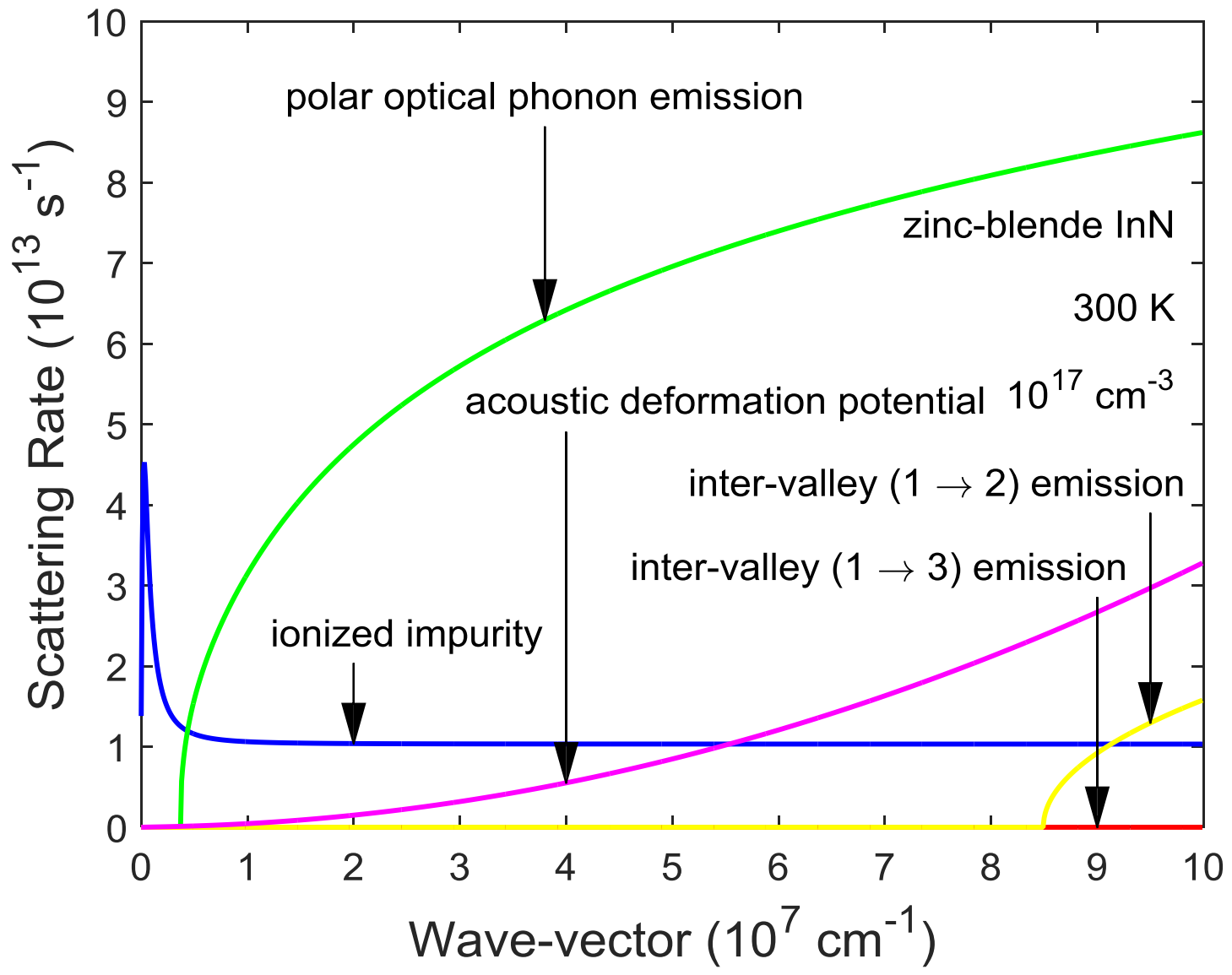

Figure 2.12.a: The scattering rates for the lowest energy $(Г)$ valley as a function of the magnitude of the wave-vector for bulk zinc-blende InN. The scattering mechanisms considered are: (1) ionized impurity (blue), (2) polar optical phonon emission (green), (3) inter-valley (1 $\rightarrow 3$ ) emission (red), (4) inter-valley $(1 \rightarrow 2$ ) emission (yellow), (5) acoustic deformation potential (magenta). This figure has been modified from (C) Siddiqua, P. \& O'Leary, S.K. (2018). Electron transport within the wurtzite and zinc-blende phases of gallium nitride and indium nitride, Journal of Materials Science, 29, 3511-3567. Page 3527. Adapted with permission from publisher. The online version of this figure is depicted in color. 


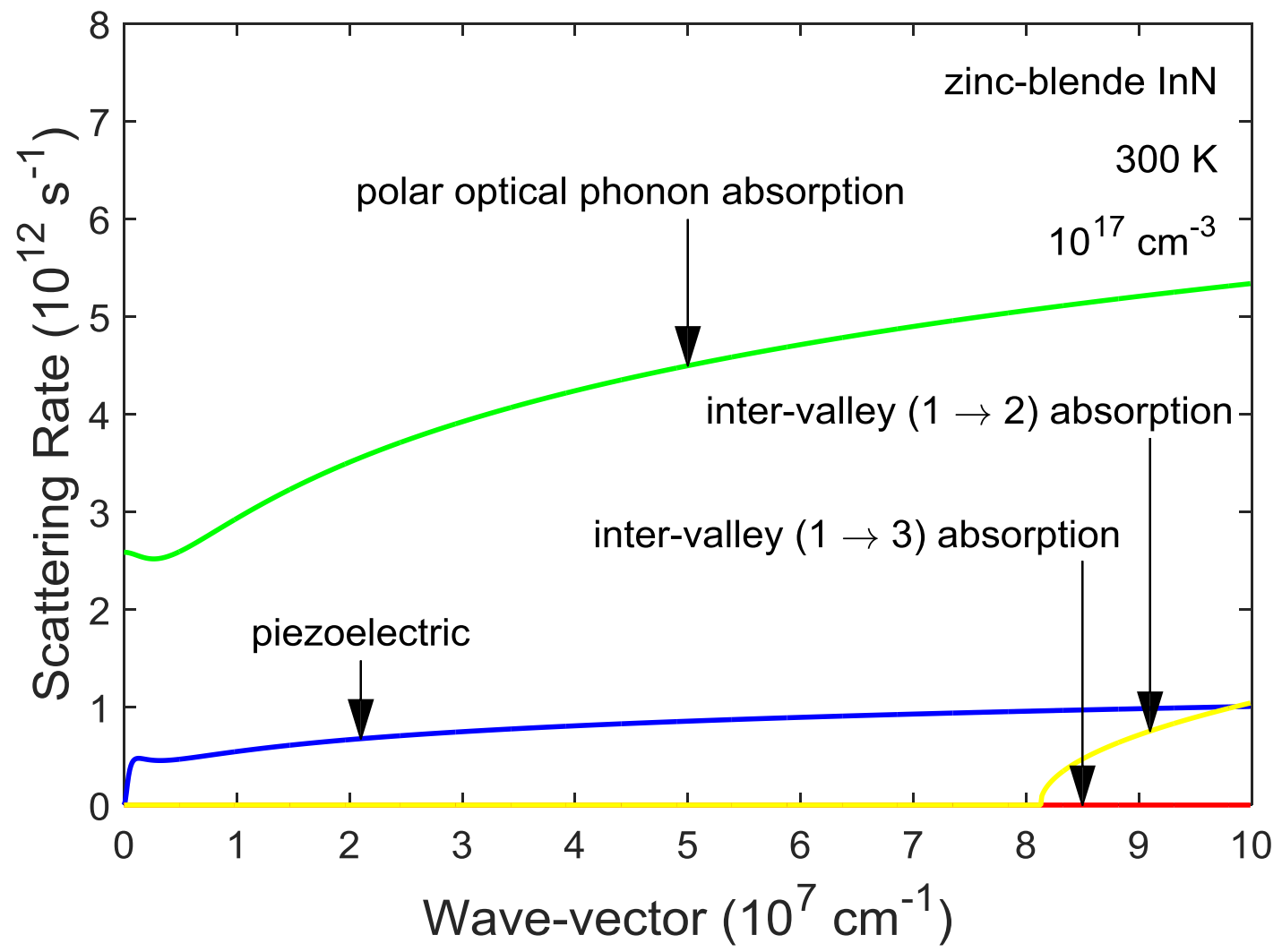

Figure 2.12.b: The scattering rates for the lowest energy $(Г)$ valley as a function of the magnitude of the wave-vector for bulk zinc-blende InN. The scattering mechanisms considered are: (1) piezoelectric (blue), (2) polar optical phonon absorption (green), (3) inter-valley $(1 \rightarrow 3)$ absorption (red), and (4) inter-valley $(1 \rightarrow 2$ ) absorption (yellow). This figure has been modified from (C Siddiqua, P. \& O'Leary, S.K. (2018). Electron transport within the wurtzite and zinc-blende phases of gallium nitride and indium nitride, Journal of Materials Science, 29, 3511-3567. Page 3527. Adapted with permission from publisher. The online version of this figure is depicted in color. 


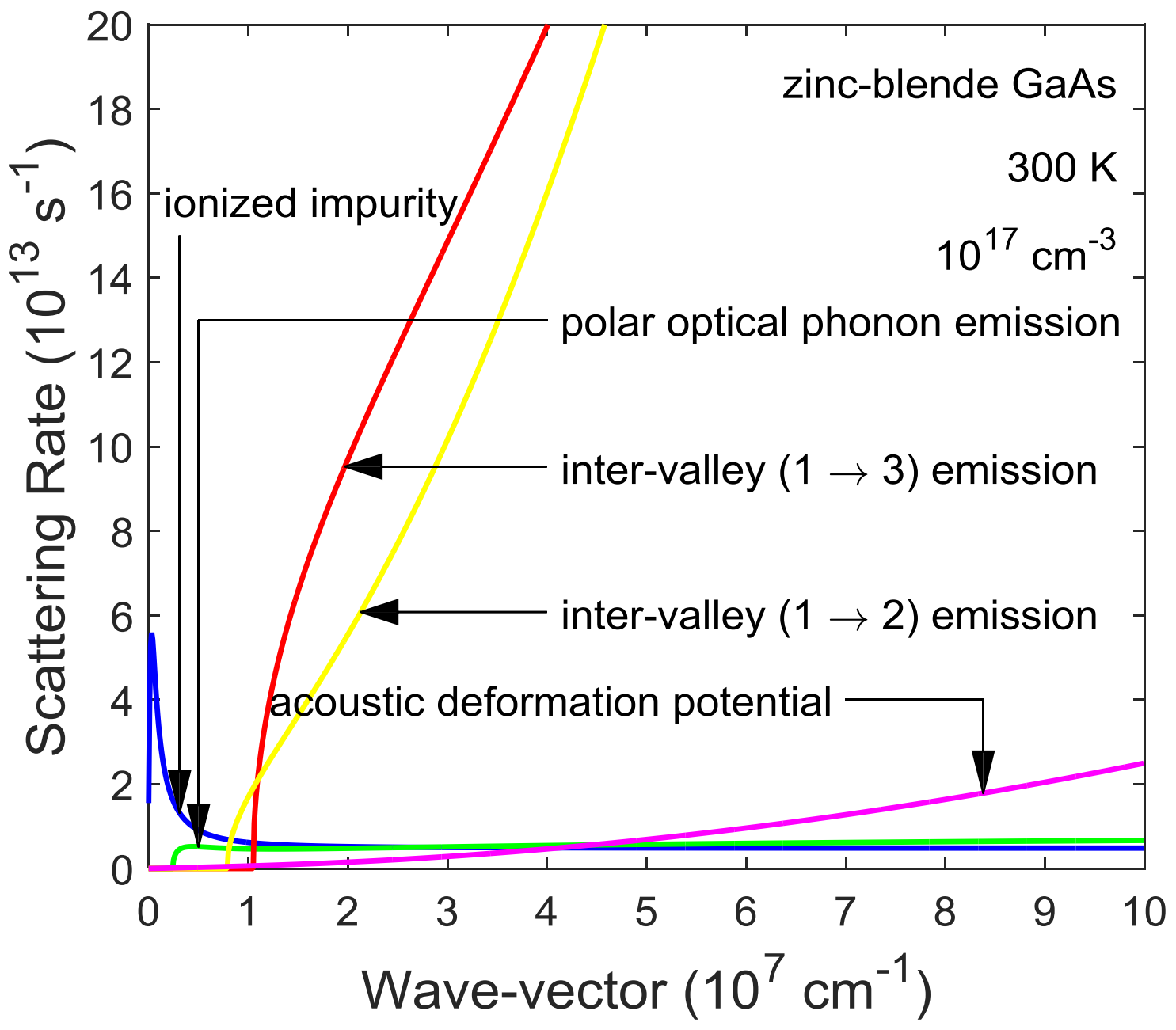

Figure 2.13.a: The scattering rates for the lowest energy $(\ulcorner)$ valley as a function of the magnitude of the wave-vector for bulk zinc-blende GaAs. The scattering mechanisms considered are: (1) ionized impurity (blue), (2) polar optical phonon emission (green), (3) inter-valley $(1 \rightarrow 3)$ emission (red), (4) inter-valley $(1 \rightarrow 2$ ) emission (yellow), (5) acoustic deformation potential (magenta). This figure has been modified from (C) Siddiqua, P. \& O'Leary, S.K. (2018). Electron transport within the wurtzite and zinc-blende phases of gallium nitride and indium nitride, Journal of Materials Science, 29, 3511-3567. Page 3528. Adapted with permission from publisher. The online version of this figure is depicted in color. 


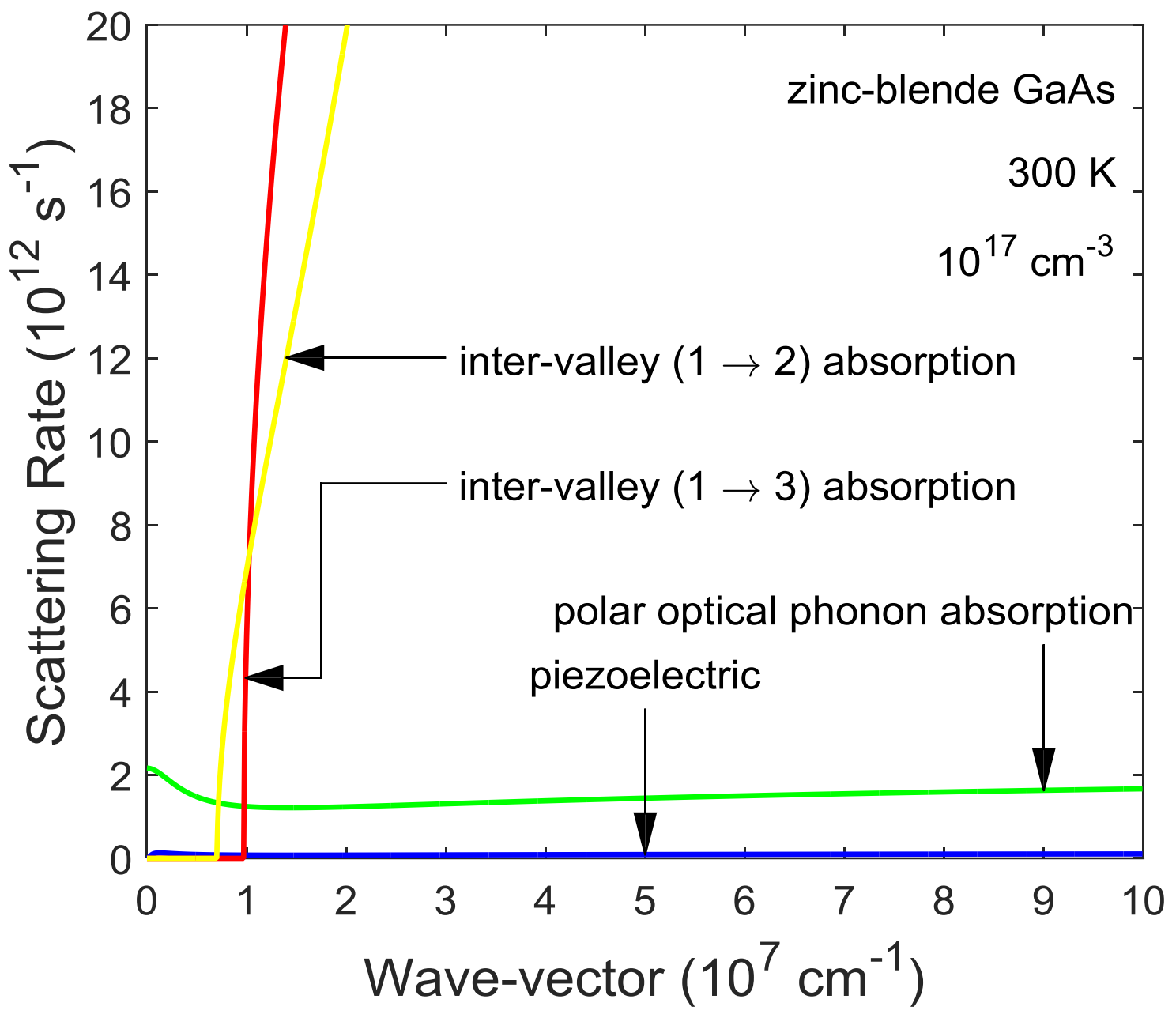

Figure 2.13.b: The scattering rates for the lowest energy $(\ulcorner)$ valley as a function of the magnitude of the wave-vector for bulk zinc-blende GaAs. The scattering mechanisms considered are: (1) piezoelectric (blue), (2) polar optical phonon absorption (green), (3) inter-valley $(1 \rightarrow 3)$ absorption (red), and (4) inter-valley $(1 \rightarrow 2$ ) absorption (yellow). This figure has been modified from ( ) Siddiqua, P. \& O’Leary, S.K. (2018). Electron transport within the wurtzite and zinc-blende phases of gallium nitride and indium nitride, Journal of Materials Science, 29, 3511-3567. Page 3528. Adapted with permission from publisher. The online version of this figure is depicted in color. 


\section{Chapter 3: Steady-state and transient electron transport results within the wurtzite and zinc-blende phases of $\mathrm{GaN}$ and $\mathrm{InN}$}

\subsection{Overview}

The current interest in the III-V nitride compound semiconductors, GaN and $\operatorname{lnN}$, is primarily being fueled by the tremendous potential of these materials for novel electronic and opto-electronic device applications. With the recognition that informed electronic and optoelectronic device design requires a firm understanding of the nature of the electron transport within these materials, electron transport within the compound semiconductors, GaN and InN, has been the focus of intensive investigation for many years. The literature abounds with studies on the steady-state and transient electron transport within these materials $[1,2,142,143]$. As a result of this intense flurry of research activity, wide energy gap compound semiconductor-based devices are being deployed in products that are widely available today [144-156]. ${ }^{15}$ Future developments in the wide energy gap compound semiconductor field will undoubtedly require an even deeper understanding of the electron transport mechanisms within these materials.

In the previous chapter, I presented details of the semi-classical three-valley Monte Carlo simulation approach, that I employ for the analysis of the electron transport within the wurtzite and zinc-blende phases of $\mathrm{GaN}$ and $\operatorname{lnN}$. In this chapter, a collection of steady-state and transient electron transport results, obtained from these Monte Carlo electron transport simulations, is presented. Initially, an overview of our steady-state electron transport results, corresponding to the semiconductors under consideration in this analysis, i.e., the wurtzite and zinc-blende phases of $\mathrm{GaN}$ and $\mathrm{InN}$, will be provided, and a comparison with the more conventional compound semi-

\footnotetext{
15 The wide energy gap semiconductors that are available today include those based on the III-V nitrides, $\mathrm{ZnO}$, silicon carbide $(\mathrm{SiC})$, and a variety of other wide energy gap semiconductors. The list of references included here include devices based on this broader class of materials.
} 
conductor, GaAs, will be presented. Later, the transient electron transport that occurs within the semiconductors under investigation in this analysis, i.e., the wurtzite and zinc-blende phases of GaN and InN, is characterized and contrasted with that corresponding to GaAs.

This chapter is organized in the following manner. In Sections 3.2, 3.3, 3.4, and 3.5, steady-state electron transport characteristics, associated with the wurtzite and zinc-blende phases of $\mathrm{GaN}$ and $\mathrm{InN}$, are presented and analysed. For bench-marking purposes, in Section 3.6, an analogous steady-state analysis is performed for the case of zinc-blende GaAs, the steady-state electron transport characteristics associated with the family of wide energy gap semiconductors under consideration in this analysis, i.e., the wurtzite and zinc-blende phases of $\mathrm{GaN}$ and $\mathrm{InN}$, being compared and contrasted with that corresponding to zinc-blende GaAs in Section 3.7. The sensitivity of these steady-state electron transport characteristics to variations in the crystal temperature and the doping concentration is then probed in Sections 3.8 and 3.9, respectively. Transient electron transport results, corresponding to the materials under investigation in this analysis, i.e., the wurtzite and zinc-blende phases of GaN and InN, and zincblende GaAs, are then featured in Section 3.10. Finally, the conclusions of this electron transport analysis are summarized in Section 3.11.

\subsection{Steady-state electron transport within wurtzite GaN}

Our examination of results begins with wurtzite GaN. The velocity-field characteristic associated with this material is depicted in Figure 3.1. This result is obtained through a steadystate Monte Carlo simulation of the electron transport within this material for the wurtzite GaN parameter selections specified in Tables 2.1 and 2.2, the crystal temperature being set to $300 \mathrm{~K}$ and the doping concentration being set to $10^{17} \mathrm{~cm}^{-3}$. We note that initially the electron drift velocity monotonically increases with the applied electric field strength, reaching a maximum of about 2.9 $\times 10^{7} \mathrm{~cm} / \mathrm{s}$ when the applied electric field strength is around $140 \mathrm{kV} / \mathrm{cm}$. For applied electric fields 
strengths in excess of $140 \mathrm{kV} / \mathrm{cm}$, the electron drift velocity decreases in response to further increases in the applied electric field strength, i.e., a region of negative differential mobility is observed, the electron drift velocity eventually saturating at about $1.4 \times 10^{7} \mathrm{~cm} / \mathrm{s}$ for sufficiently high applied electric field strengths.

We now focus on the results at low applied electric field strengths, i.e., applied electric field strengths less than $10 \mathrm{kV} / \mathrm{cm}$. This is referred to as the linear regime of electron transport, as in this regime, the electron drift velocity is well characterized by the low-field electron drift mobility, $\mu$, i.e., a linear low-field electron drift velocity dependence on the applied electric field strength, $v_{d}=\mu E$, applies in this regime. Examining the distribution function for this regime, we find that it is very similar to the zero-field distribution function with a slight shift in the direction opposite of the applied electric field; this arises as electrons are negatively charged. In this regime, the average electron energy remains relatively low, with most of the energy gained from the applied electric field being transferred into the lattice through polar optical phonon scattering. We find that the low-field electron drift mobility, $\mu$, corresponding to the velocity-field characteristic depicted in Figure 3.1 , is around $850 \mathrm{~cm}^{2} / \mathrm{V} . \mathrm{s}$.

If we now examine the average electron energy as a function of the applied electric field strength, shown in Figure 3.2, we see that there is a sudden increase at around $100 \mathrm{kV} / \mathrm{cm}$; this result is obtained from the same steady-state wurtzite GaN Monte Carlo simulation of electron transport as that used to determine Fig. 3.1, the crystal temperature being set to $300 \mathrm{~K}$ and the doping concentration being set to $10^{17} \mathrm{~cm}^{-3}$. In order to understand why this increase occurs, we note that the dominant energy loss mechanism for many compound semiconductors, including wurtzite $\mathrm{GaN}$, is polar optical phonon scattering. When the applied electric field strength is less than $100 \mathrm{kV} / \mathrm{cm}$, all of the energy that the electrons gain from the applied electric field is lost through polar optical phonon scattering. The other scattering mechanisms, i.e., ionized impurity scattering, piezoelectric scattering, and acoustic deformation potential scattering, do not remove 
energy from the electron ensemble, i.e., they are elastic scattering mechanisms. Beyond a certain critical applied electric field strength, however, and the polar optical phonon scattering mechanism can no longer remove all of the energy gained from the applied electric field. Other scattering mechanisms must start to play a role if the electron ensemble is to remain in equilibrium. The average electron energy increases until inter-valley scattering begins and an energy balance is re-established; the energy levels of the two lowest energy upper conduction band minima corresponding to this material, i.e., the two lowest upper conduction band energy valley minima, are depicted in Figure 3.2.

As the applied electric field strength is increased beyond $100 \mathrm{kV} / \mathrm{cm}$, the average electron energy increases until a substantial fraction of the electrons have acquired enough energy in order to transfer into the upper energy conduction band valleys. In Figure 3.3, we plot the occupancy of the three lowest energy conduction band valleys as a function of the applied electric field strength for the case of wurtzite GaN, this result being obtained from the same steady-state wurtzite GaN Monte Carlo simulation of electron transport as that used to determine Figures 3.1 and 3.2, the motion of three-thousand electrons being considered for this analysis, the crystal temperature being set to $300 \mathrm{~K}$ and the doping concentration being set to $10^{17} \mathrm{~cm}^{-3}$. As the effective mass of the electrons in the upper energy conduction band valleys is greater than that in the lowest energy conduction band valley, the electrons in the upper energy conduction band valleys will be slower. As more electrons transfer to the upper energy conduction band valleys, the electron drift velocity decreases. This accounts for the negative differential mobility observed in the velocity-field characteristic depicted in Figure 3.1.

Finally, at sufficiently high applied electric field strengths, the number of electrons in each conduction band valley saturates. It can be shown that in the high-field limit, the number of electrons in each conduction band valley is proportional to the product of the density of states of 


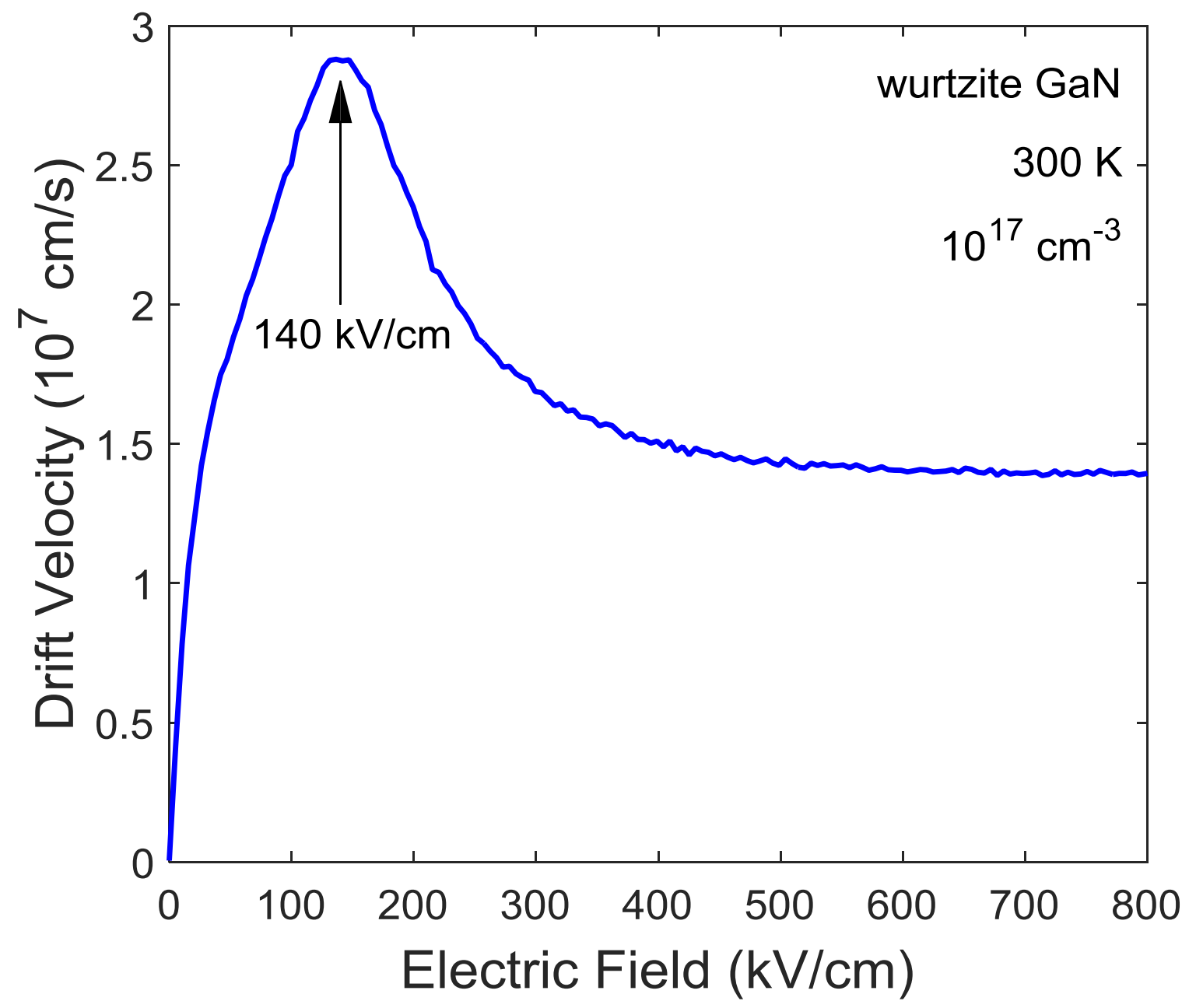

Figure 3.1: The velocity-field characteristic associated with bulk wurtzite GaN for the crystal temperature set to $300 \mathrm{~K}$ and the doping concentration set to $10^{17} \mathrm{~cm}^{-3}$. Like many other compound semiconductors, the electron drift velocity reaches a peak, and at higher applied electric field strengths it decreases until it saturates. The peak field, i.e., the applied electric field strength at which the maximum electron drift velocity occurs, $140 \mathrm{kV} / \mathrm{cm}$, is clearly indicated with an arrow. This figure has been modified from @ Siddiqua, P. \& O'Leary, S.K. (2018). Electron transport within the wurtzite and zinc-blende phases of gallium nitride and indium nitride, Journal of Materials Science, 29, 3511-3567. Page 3528. Adapted with permission from publisher. The online version of this figure is depicted in color. 


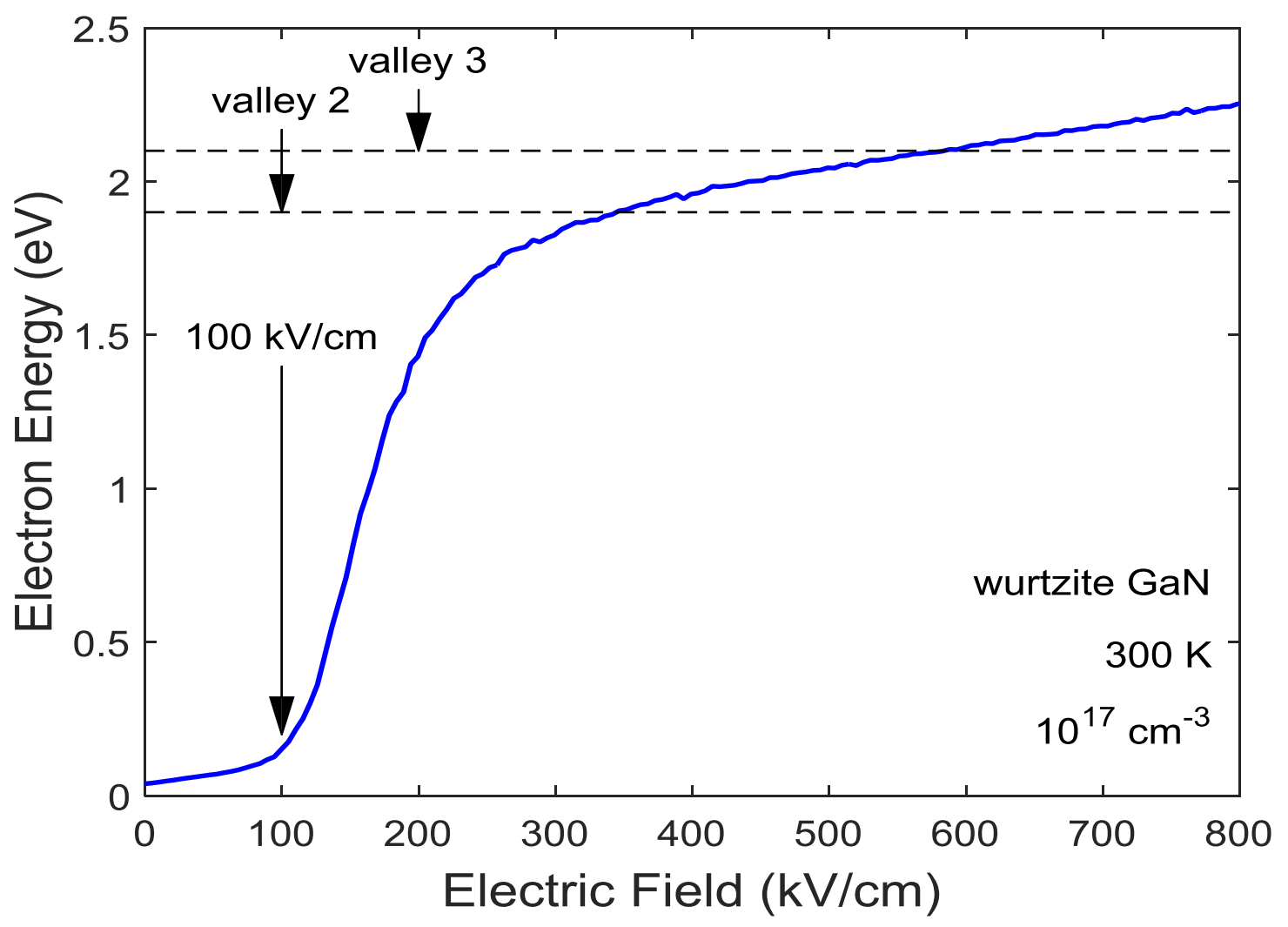

Figure 3.2: The average electron energy as a function of the applied electric field strength for bulk wurtzite GaN for the crystal temperature set to $300 \mathrm{~K}$ and the doping concentration set to $10^{17} \mathrm{~cm}^{-3}$. Initially, the average electron energy remains low, only slightly higher than the thermal energy, $3 / 2 k_{B} T$, where $k_{B}$ denotes the Boltzmann constant. At $100 \mathrm{kV} / \mathrm{cm}$, however, the average electron energy increases dramatically. This increase is due to the fact that the polar optical phonon scattering mechanism can no longer absorb all of the energy gained from the applied electric field. The energy minima corresponding to the lowest and second lowest upper conduction band valley minima are depicted with the dashed lines. This figure has been modified from (C) Siddiqua, P. \& O'Leary, S.K. (2018). Electron transport within the wurtzite and zinc-blende phases of gallium nitride and indium nitride, Journal of Materials Science, 29, 3511-3567. Page 3529. Adapted with permission from publisher. The online version of this figure is depicted in color. 


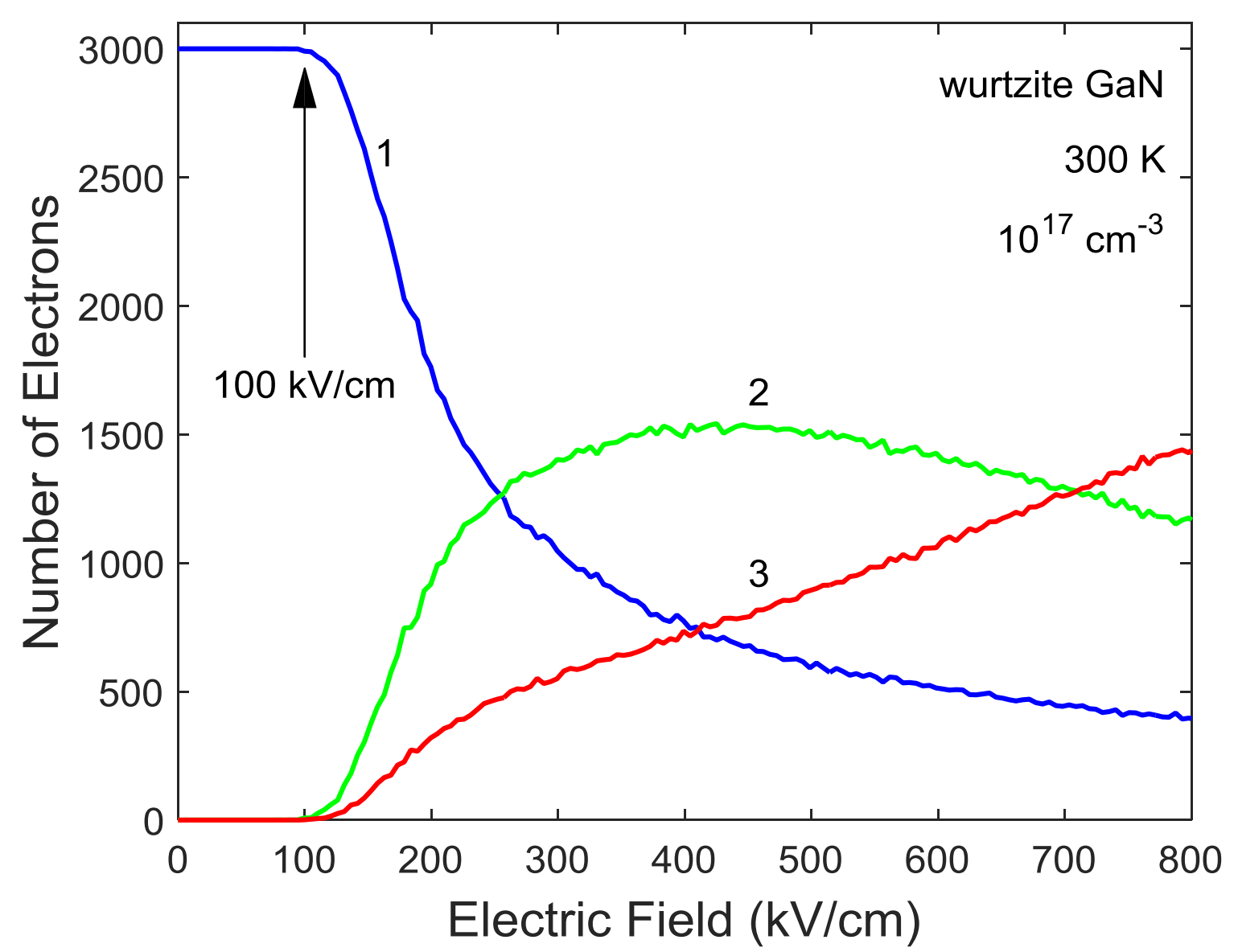

Figure 3.3: The valley occupancy as a function of the applied electric field strength for the case of bulk wurtzite GaN for the crystal temperature set to $300 \mathrm{~K}$ and the doping concentration set to $10^{17} \mathrm{~cm}^{-3}$. Soon after the average electron energy increases, i.e., at about $100 \mathrm{kV} / \mathrm{cm}$, electrons begin to transfer to the upper valleys of the conduction band. There were three-thousand electrons employed for this simulation. The valleys are labeled 1,2, and 3, in accordance with their energy minima, i.e., the lowest energy conduction band valley minimum corresponding to valley 1 , the second lowest energy conduction band valley minimum corresponding to valley 2 , the third lowest energy conduction band valley minimum corresponding to valley 3. This figure has been modified from (C) Siddiqua, P. \& O'Leary, S.K. (2018). Electron transport within the wurtzite and zinc-blende phases of gallium nitride and indium nitride, Journal of Materials Science, 29, 3511-3567. Page 3529. Adapted with permission from publisher. The online version of this figure is depicted in color. 
that particular valley and the corresponding valley degeneracy. At this point, the electron drift velocity stops decreasing and achieves saturation.

\subsection{Steady-state electron transport within zinc-blende GaN}

I continue my analysis with an examination of the steady-state electron transport within zinc-blende GaN. The velocity-field characteristic associated with this material is depicted in Figure 3.4. This result is obtained through a steady-state Monte Carlo simulation of the electron transport within this material for the zinc-blende GaN parameter selections specified in Tables 2.1 and 2.2, the crystal temperature being set to $300 \mathrm{~K}$ and the doping concentration being set to $10^{17}$ $\mathrm{cm}^{-3}$. We note that initially the electron drift velocity monotonically increases with the applied electric field strength, reaching a maximum of about $3.3 \times 10^{7} \mathrm{~cm} / \mathrm{s}$ when the applied electric field strength is around $110 \mathrm{kV} / \mathrm{cm}$. As with the case of wurtzite $\mathrm{GaN}$, a linear regime of electron transport is observed, the low-field electron drift mobility, $\mu$, corresponding to the velocity-field characteristic depicted in Figure 3.4, being about $1250 \mathrm{~cm}^{2} / \mathrm{V}$.s. For applied electric fields strengths in excess of $110 \mathrm{kV} / \mathrm{cm}$, the electron drift velocity decreases in response to further increases in the applied electric field strength, i.e., a region of negative differential mobility is observed, the electron drift velocity eventually saturating at about $2.1 \times 10^{7} \mathrm{~cm} / \mathrm{s}$ for sufficiently high applied electric field strengths; it should be noted that this saturation occurs beyond the range of electric field strengths depicted in Figure 3.4.

If we examine the average electron energy as a function of the applied electric field strength, shown in Figure 3.5, we see that there is a sudden increase at around $80 \mathrm{kV} / \mathrm{cm}$; this result was obtained from the same steady-state zinc-blende GaN Monte Carlo simulation of electron transport as that used to determine Figure 3.4, the crystal temperature being set to 300 $\mathrm{K}$ and the doping concentration being set to $10^{17} \mathrm{~cm}^{-3}$. As with the case of wurtzite $\mathrm{GaN}$, beyond 


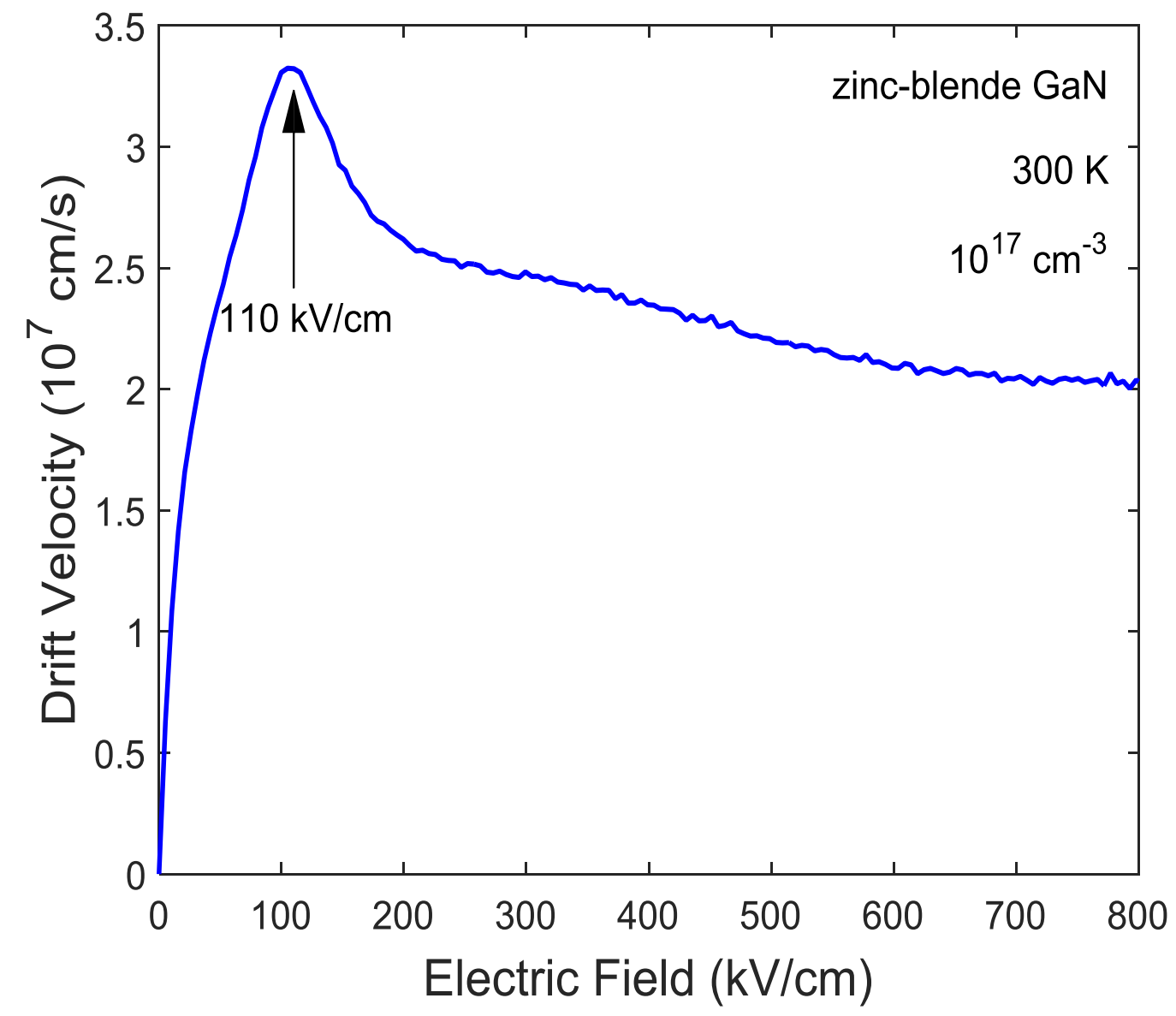

Figure 3.4: The velocity-field characteristic associated with bulk zinc-blende GaN for the crystal temperature set to $300 \mathrm{~K}$ and the doping concentration set to $10^{17} \mathrm{~cm}^{-3}$. Like many other compound semiconductors, the electron drift velocity reaches a peak, and at higher applied electric field strengths it decreases until it saturates. The peak field, i.e., the applied electric field strength at which the maximum electron drift velocity occurs, $110 \mathrm{kV} / \mathrm{cm}$, is clearly indicated with an arrow. This figure has been modified from () Siddiqua, P. \& O'Leary, S.K. (2018). Electron transport within the wurtzite and zinc-blende phases of gallium nitride and indium nitride, Journal of Materials Science, 29, 3511-3567. Page 3530. Adapted with permission from publisher. The online version of this figure is depicted in color. 


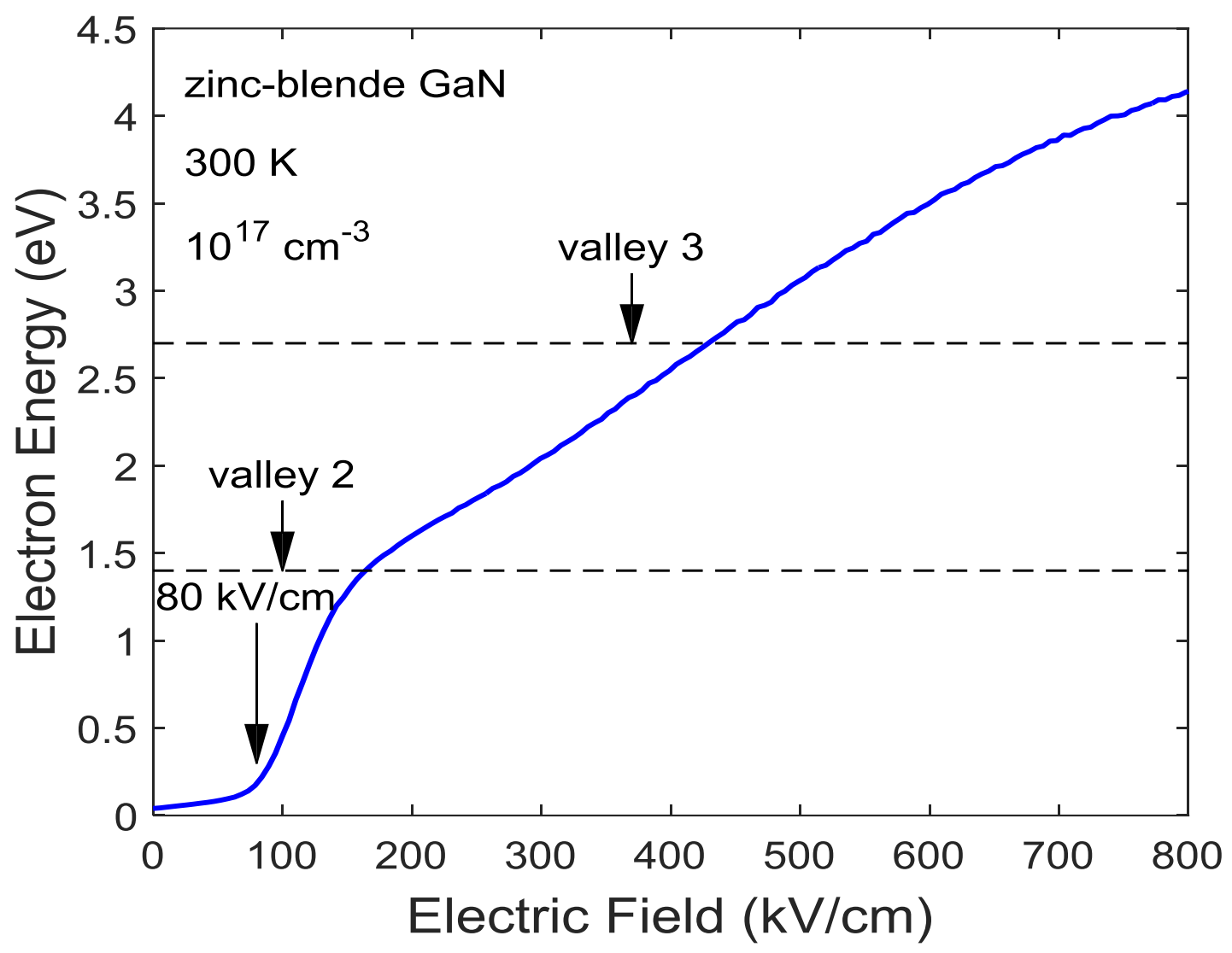

Figure 3.5: The average electron energy as a function of the applied electric field strength for bulk zincblende GaN for the crystal temperature set to $300 \mathrm{~K}$ and the doping concentration set to $10^{17} \mathrm{~cm}^{-3}$. Initially, the average electron energy remains low, only slightly higher than the thermal energy, $3 / 2 k_{B} T$, where $k_{B}$ denotes the Boltzmann constant. At $80 \mathrm{kV} / \mathrm{cm}$, however, the average electron energy increases dramatically. This increase is due to the fact that the polar optical phonon scattering mechanism can no longer absorb all of the energy gained from the applied electric field. The energy minima corresponding to the lowest and the second lowest upper conduction band valley minima are depicted with the dashed lines. This figure has been modified from (C Siddiqua, P. \& O'Leary, S.K. (2018). Electron transport within the wurtzite and zinc-blende phases of gallium nitride and indium nitride, Journal of Materials Science, 29, 3511-3567. Page 3530. Adapted with permission from publisher. The online version of this figure is depicted in color. 


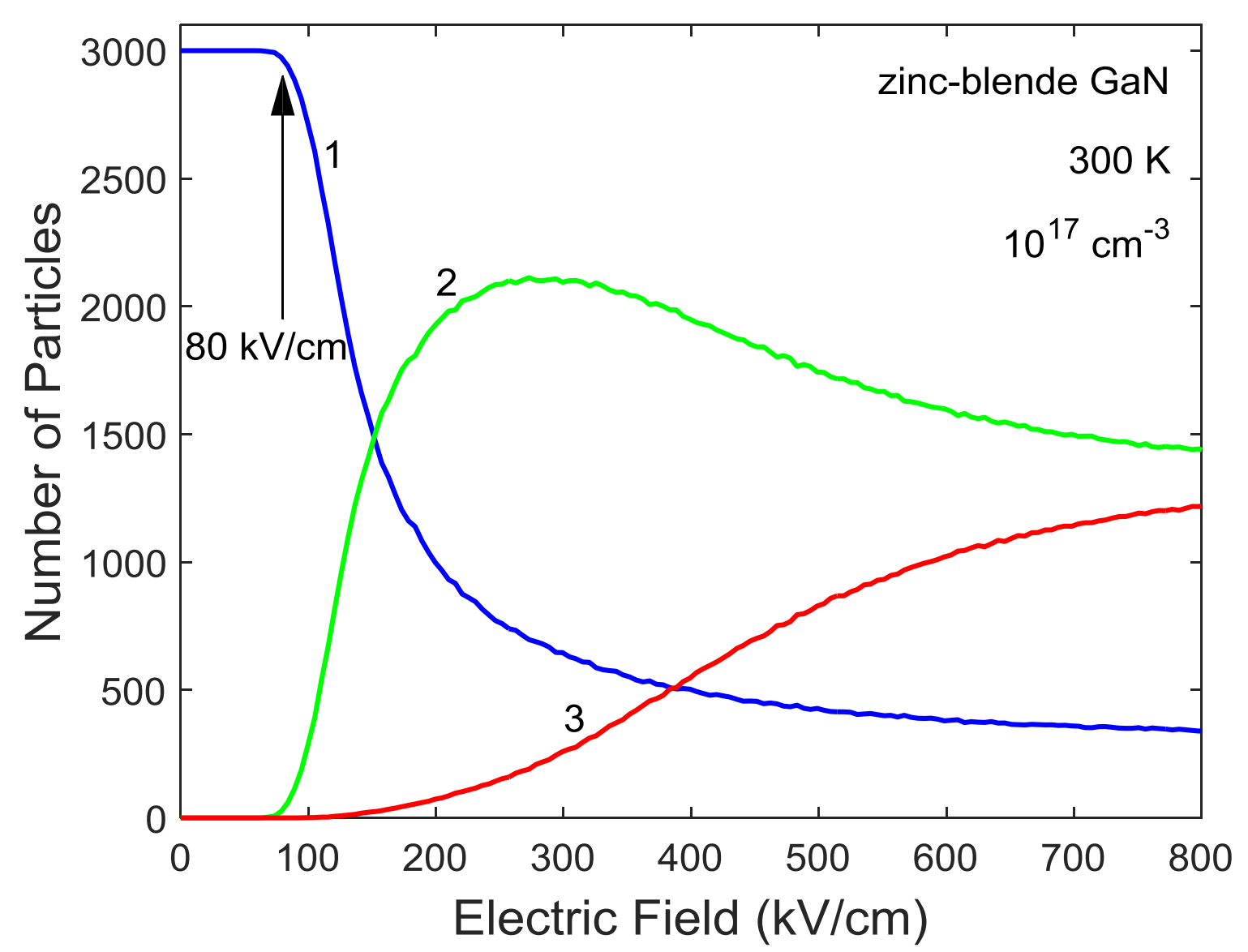

Figure 3.6: The valley occupancy as a function of the applied electric field strength for the case of bulk zinc-blende $\mathrm{GaN}$ for the crystal temperature set to $300 \mathrm{~K}$ and the doping concentration set to $10^{17} \mathrm{~cm}^{-3}$. Soon after the average electron energy increases, i.e., at about $80 \mathrm{kV} / \mathrm{cm}$, electrons begin to transfer to the upper valleys of the conduction band. There were three-thousand electrons employed for this simulation. The valleys are labeled 1,2, and 3, in accordance with their energy minima, i.e., the lowest energy conduction band valley minimum corresponding to valley 1 , the second lowest energy conduction band valley minimum corresponding to valley 2 , the third lowest energy conduction band valley minimum corresponding to valley 3. This figure has been modified from @ (c Siddiqua, P. \& O'Leary, S.K. (2018). Electron transport within the wurtzite and zinc-blende phases of gallium nitride and indium nitride, Journal of Materials Science, 29, 3511-3567. Page 3531. Adapted with permission from publisher. The online version of this figure is depicted in color. 
a certain critical applied electric field strength, polar optical phonon scattering can no longer remove all of the energy gained from the applied electric field. The average electron energy increases until inter-valley scattering begins and an energy balance is re-established; the energy levels of the two lowest energy upper conduction band minima corresponding to this material, i.e., the two lowest energy upper conduction band valleys, are depicted in Figure 3.5.

In Figure 3.6, we plot the occupancy of the valleys as a function of the applied electric field strength for the case of zinc-blende GaN, this result being obtained from the same steady-state zinc-blende GaN Monte Carlo simulation of electron transport as that used to determine Figures 3.4 and 3.5, the motion of three-thousand electrons being considered for this steady-state electron transport analysis, the crystal temperature being set to $300 \mathrm{~K}$ and the doping concentration being set to $10^{17} \mathrm{~cm}^{-3}$. This result is similar in character to that found for the case of wurtzite GaN.

\subsection{Steady-state electron transport within wurtzite $\ln N$}

I now examine the steady-state electron transport within wurtzite $\ln N$. The electrons within InN have a lower effective mass than those associated with GaN. The velocity-field characteristic associated with this material is depicted in Figure 3.7. This result is obtained through a steadystate Monte Carlo simulation of the electron transport within this material for the wurtzite $\ln N$ parameter selections specified in Tables 2.1 and 2.2, the crystal temperature being set to $300 \mathrm{~K}$ and the doping concentration being set to $10^{17} \mathrm{~cm}^{-3}$. We note that initially the electron drift velocity monotonically increases with the applied electric field strength, reaching a maximum of about 5.6 $\times 10^{7} \mathrm{~cm} / \mathrm{s}$ when the applied electric field strength is around $30 \mathrm{kV} / \mathrm{cm}$. As with the cases of wurtzite and zinc-blende GaN, a linear regime of electron transport is observed for the case of wurtzite $\operatorname{lnN}$, the low-field electron drift mobility, $\mu$, corresponding to the velocity-field characteristic depicted in Figure 3.7, being about $8700 \mathrm{~cm}^{2} /$ V.s . For applied electric fields strengths in excess of $30 \mathrm{kV} / \mathrm{cm}$, the electron drift velocity decreases in response to further increases in the applied electric field strength, i.e., a region of negative differential mobility is observed, the electron drift 
velocity eventually saturating at about $1.2 \times 10^{7} \mathrm{~cm} / \mathrm{s}$ for sufficiently high applied electric field strengths; it should be noted that this saturation occurs beyond the range of electric field strengths depicted in Figure 3.7.

If we examine the average electron energy as a function of the applied electric field strength, shown in Figure 3.8, we see that there is a sudden increase at around $25 \mathrm{kV} / \mathrm{cm}$; this result was obtained from the same wurtzite InN Monte Carlo simulation of electron transport as that used to determine Figure 3.7. As with the cases of wurtzite and zinc-blende GaN, beyond a certain critical applied electric field strength, polar optical phonon scattering can no longer remove all of the energy gained from the applied electric field. The average electron energy increases until inter-valley scattering begins and an energy balance is re-established.

In Figure 3.9, we plot the occupancy of the three lowest energy conduction band valleys as a function of the applied electric field strength for the case of wurtzite $\ln N$, this result being obtained from the same wurtzite InN Monte Carlo simulation of electron transport as that used to determine Figures 3.7 and 3.8, the motion of three-thousand electrons being considered for this steady-state electron transport analysis. This result is similar to that found for the cases of wurtzite and zinc-blende GaN.

\subsection{Steady-state electron transport within zinc-blende InN}

We now examine the steady-state electron transport within zinc-blende $\ln N$. The velocityfield characteristic associated with this material is depicted in Figure 3.10. This result is obtained through a Monte Carlo simulation of the electron transport within this material for the zinc-blende InN parameter selections specified in Tables 2.1 and 2.2, the crystal temperature being set to 300 


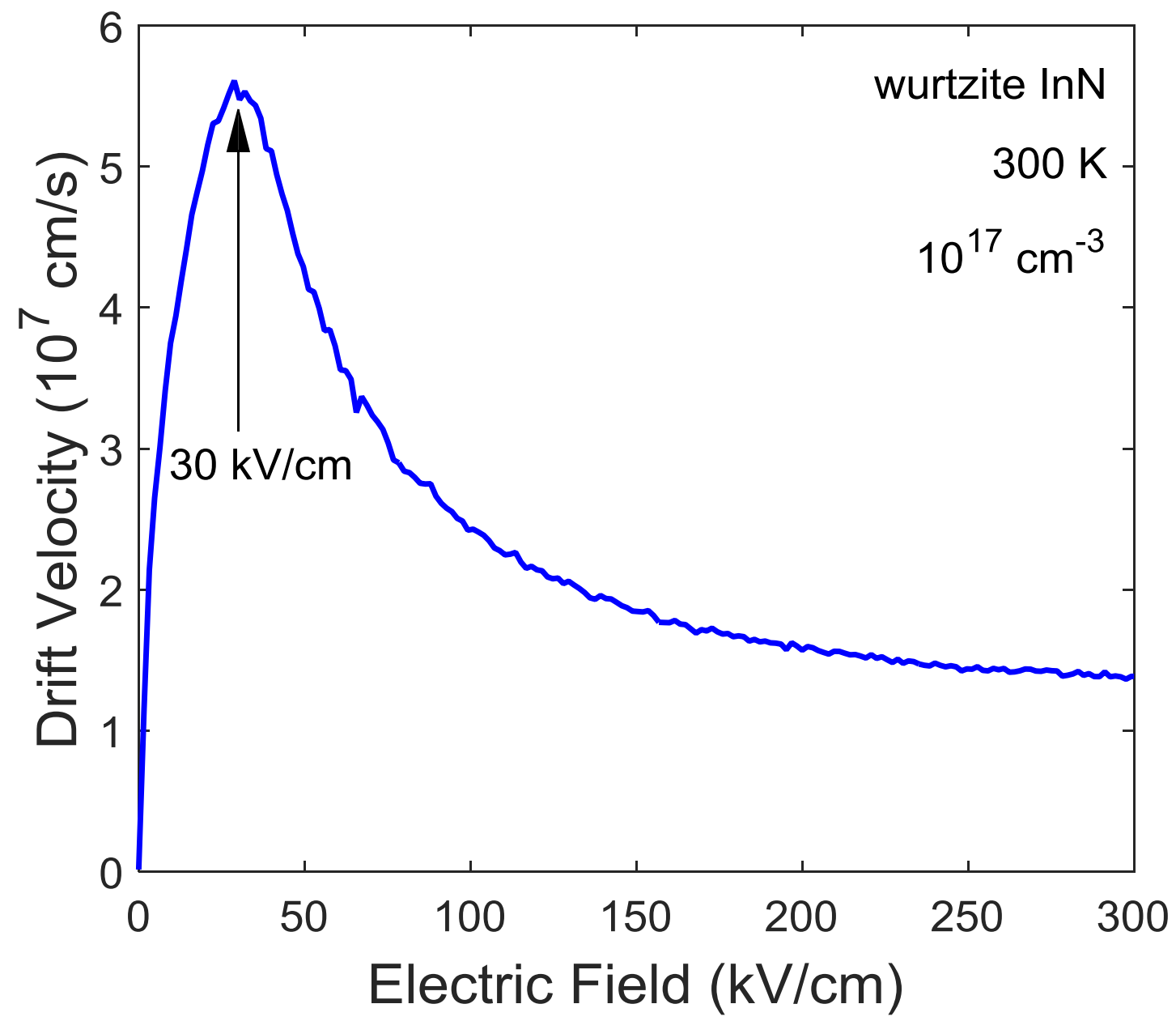

Figure 3.7: The velocity-field characteristic associated with bulk wurtzite $\ln N$ for the crystal temperature set to $300 \mathrm{~K}$ and the doping concentration set to $10^{17} \mathrm{~cm}^{-3}$. Like many other compound semiconductors, the electron drift velocity reaches a peak, and at higher applied electric field strengths it decreases until it saturates. The peak field, i.e., the applied electric field strength at which the maximum electron drift velocity occurs, $30 \mathrm{kV} / \mathrm{cm}$, is clearly indicated with an arrow. This figure has been modified from @ Siddiqua, P. \& O'Leary, S.K. (2018). Electron transport within the wurtzite and zinc-blende phases of gallium nitride and indium nitride, Journal of Materials Science, 29, 3511-3567. Page 3531. Adapted with permission from publisher. The online version of this figure is depicted in color. 


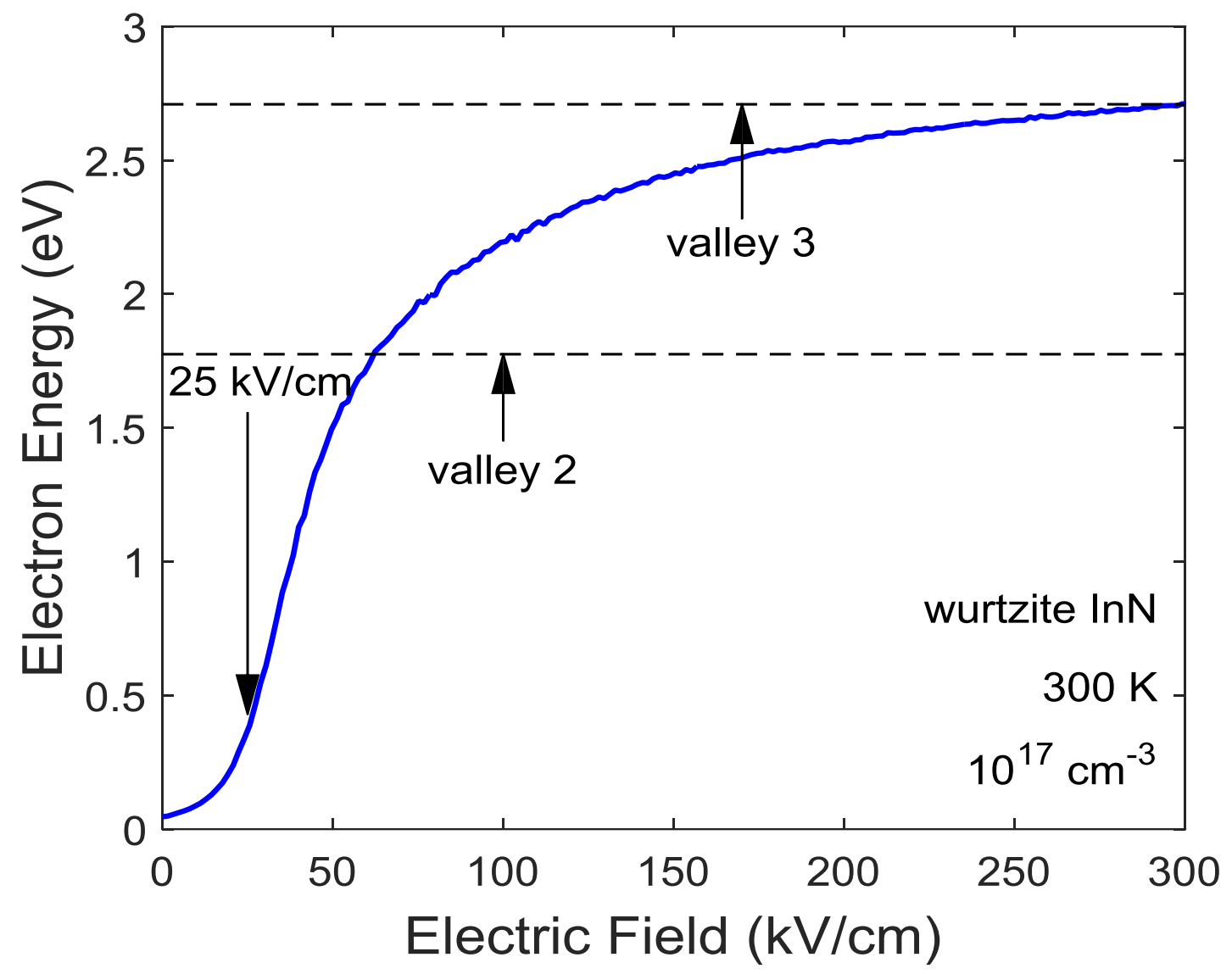

Figure 3.8: The average electron energy as a function of the applied electric field strength for bulk wurtzite InN for the crystal temperature set to $300 \mathrm{~K}$ and the doping concentration set to $10^{17} \mathrm{~cm}^{-3}$. Initially, the average electron energy remains low, only slightly higher than the thermal energy, $3 / 2 k_{B} T$, where $k_{B}$ denotes the Boltzmann constant. At $25 \mathrm{kV} / \mathrm{cm}$, however, the average electron energy increases dramatically. This increase is due to the fact that the polar optical phonon scattering mechanism can no longer absorb all of the energy gained from the applied electric field. The energy minima corresponding to the lowest and second lowest upper conduction band valley minima are depicted with the dashed lines. This figure has been modified from (C) Siddiqua, P. \& O'Leary, S.K. (2018). Electron transport within the wurtzite and zinc-blende phases of gallium nitride and indium nitride, Journal of Materials Science, 29, 3511-3567. Page 3531. Adapted with permission from publisher. The online version of this figure is depicted in color. 


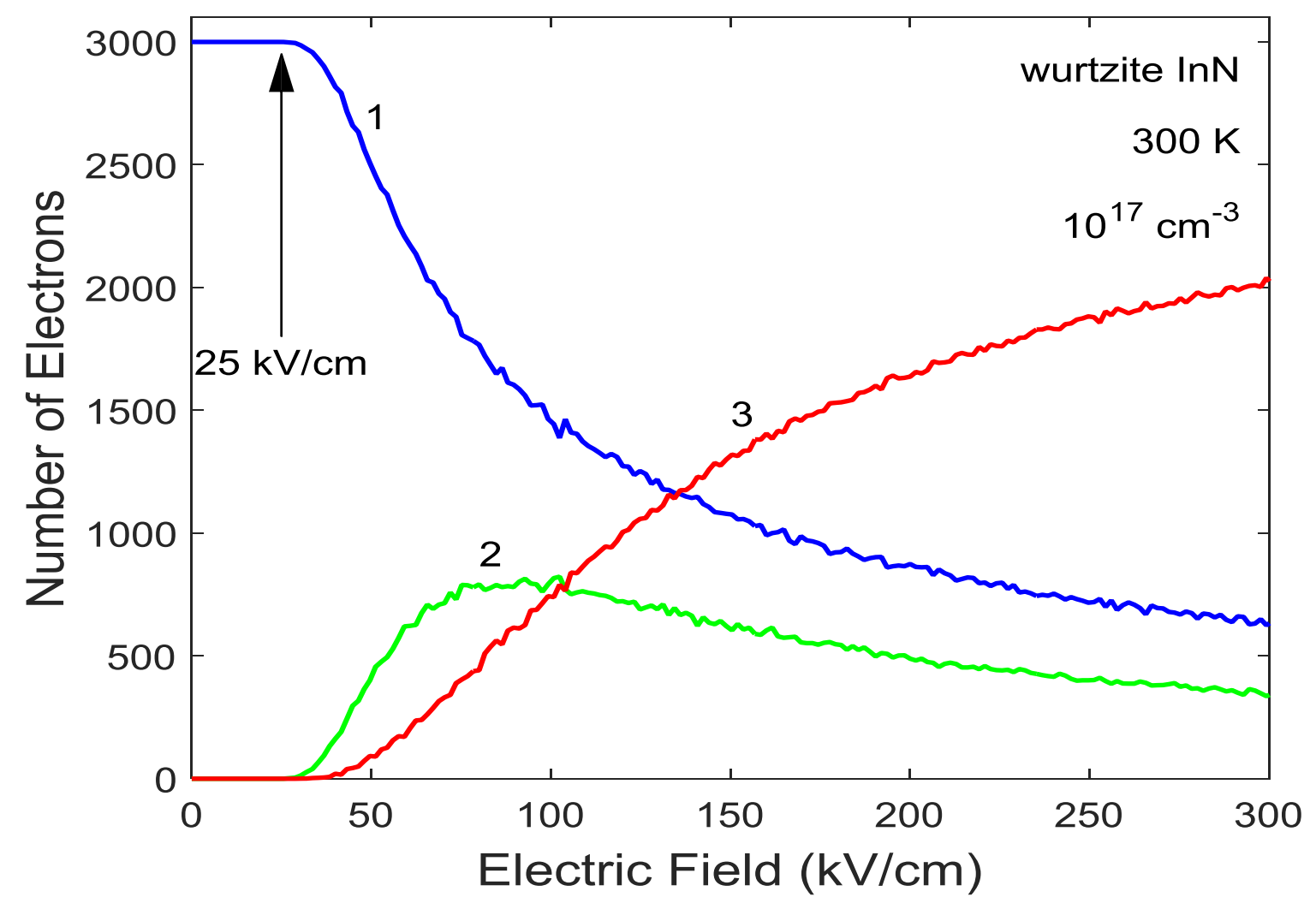

Figure 3.9: The valley occupancy as a function of the applied electric field strength for the case of bulk wurtzite $\mathrm{InN}$ for the crystal temperature set to $300 \mathrm{~K}$ and the doping concentration set to $10^{17} \mathrm{~cm}^{-3}$. Soon after the average electron energy increases, i.e., at about $25 \mathrm{kV} / \mathrm{cm}$, electrons begin to transfer to the upper valleys of the conduction band. There were three-thousand electrons employed for this simulation. The valleys are labeled 1, 2, and 3, in accordance with their energy minima, i.e., the lowest energy conduction band valley minimum corresponding to valley 1 , the second lowest energy conduction band valley minimum corresponding to valley 2 , the third lowest energy conduction band valley minimum corresponding to valley 3. This figure has been modified from @ S Siddiqua, P. \& O'Leary, S.K. (2018). Electron transport within the wurtzite and zinc-blende phases of gallium nitride and indium nitride, Journal of Materials Science, 29, 3511-3567. Page 3532. Adapted with permission from publisher. The online version of this figure is depicted in color. 
$\mathrm{K}$ and the doping concentration being set to $10^{17} \mathrm{~cm}^{-3}$. We note that initially, the electron drift velocity monotonically increases with the applied electric field strength, reaching a maximum of about $3.3 \times 10^{7} \mathrm{~cm} / \mathrm{s}$ when the applied electric field strength is around $50 \mathrm{kV} / \mathrm{cm}$. For applied electric fields strengths in excess of $50 \mathrm{kV} / \mathrm{cm}$, the electron drift velocity decreases in response to further increases in the applied electric field strength, i.e., a region of negative differential mobility is observed, the electron drift velocity eventually saturating at about $1.1 \times 10^{7} \mathrm{~cm} / \mathrm{s}$ for sufficiently high applied electric field strengths; it should be noted that this saturation occurs beyond the range of electric field strengths depicted in Figure 3.10. As with the cases of wurtzite and zinc-blende $\mathrm{GaN}$, and wurtzite $\operatorname{InN}$, a linear regime of electron transport is observed for the case of zinc-blende $\operatorname{lnN}$, the low-field electron drift mobility, corresponding to the velocity-field characteristic depicted in Figure 3.10, being about $4400 \mathrm{~cm}^{2} / \mathrm{V} . \mathrm{s}$.

If we examine the average electron energy as a function of the applied electric field strength, as shown in Figure 3.11, we see that there is a sudden increase at around $50 \mathrm{kV} / \mathrm{cm}$; this result is obtained from the same steady-state zinc-blende InN Monte Carlo simulation of electron transport as that used to determine Figure 3.10, the crystal temperature being set to 300 $\mathrm{K}$ and the doping concentration being set to $10^{17} \mathrm{~cm}^{-3}$. For the case of this material, however, the increase in the average electron energy does not saturate once the average energy approaches the conduction band inter-valley energy separation. This is because the non-parabolicity of the lowest energy conduction band valley is extremely large. This large non-parabolicity of the lowest energy conduction band valley acts to dramatically enhance the effective mass of the higher energy electrons, and this is the primary factor responsible for the negative differential mobility exhibited by the velocity-field characteristic associated with zinc-blende InN seen in Figure 3.10 [157]. 
The large non-parabolicity of the lowest energy conduction band valley limits the number of inter-valley transitions that can occur within zinc-blende InN. In Figure 3.12, we plot the occupancy of the three lowest energy conduction band valleys as a function of the applied electric field strength for the case of zinc-blende $\ln N$, this result being obtained from the same steadystate zinc-blende $\operatorname{InN}$ Monte Carlo simulation of electron transport as that used to determine Figures 3.10 and 3.11, the motion of three-thousand electrons being considered for this electron transport analysis, the crystal temperature being set to $300 \mathrm{~K}$ and the doping concentration being set to $10^{17} \mathrm{~cm}^{-3}$. It is seen that the upper conduction band valley occupancy is negligible, even for very high applied electric field strengths. This contrasts rather dramatically with the cases of the wurtzite and zinc-blende phases of $\mathrm{GaN}$ and the case of wurtzite InN. The large non-parabolicity of the lowest energy conduction band valley is the principal factor responsible for this effect [157]. ${ }^{16}$

\subsection{Steady-state electron transport within zinc-blende GaAs}

For bench-marking purposes, we now study the steady-state electron transport that occurs within zinc-blende GaAs. The velocity-field characteristic associated with this material is depicted in Figure 3.13. This result is obtained through a steady-state Monte Carlo simulation of the electron transport within this material for the zinc-blende GaAs parameter selections specified by Littlejohn et al. [116] and Blakemore [124], the crystal temperature being set to $300 \mathrm{~K}$ and the do-

16 Using a one-dimensional band structural evaluation for the effective mass of the electrons described using the Kane model, i.e., Eq. (2.2), Siddiqua et al. [157] demonstrated the important role that the nonparabolicity plays in influencing the effective mass of the electrons in a valley. The non-parabolicity associated with the lowest energy valley of the conduction band of zinc-blende $\ln N$ is significantly greater than that associated with the other materials considered in this analysis, and hence, the nature of the electron transport is expected to be quite distinct. Further details are discussed by Siddiqua et al. [157]. 


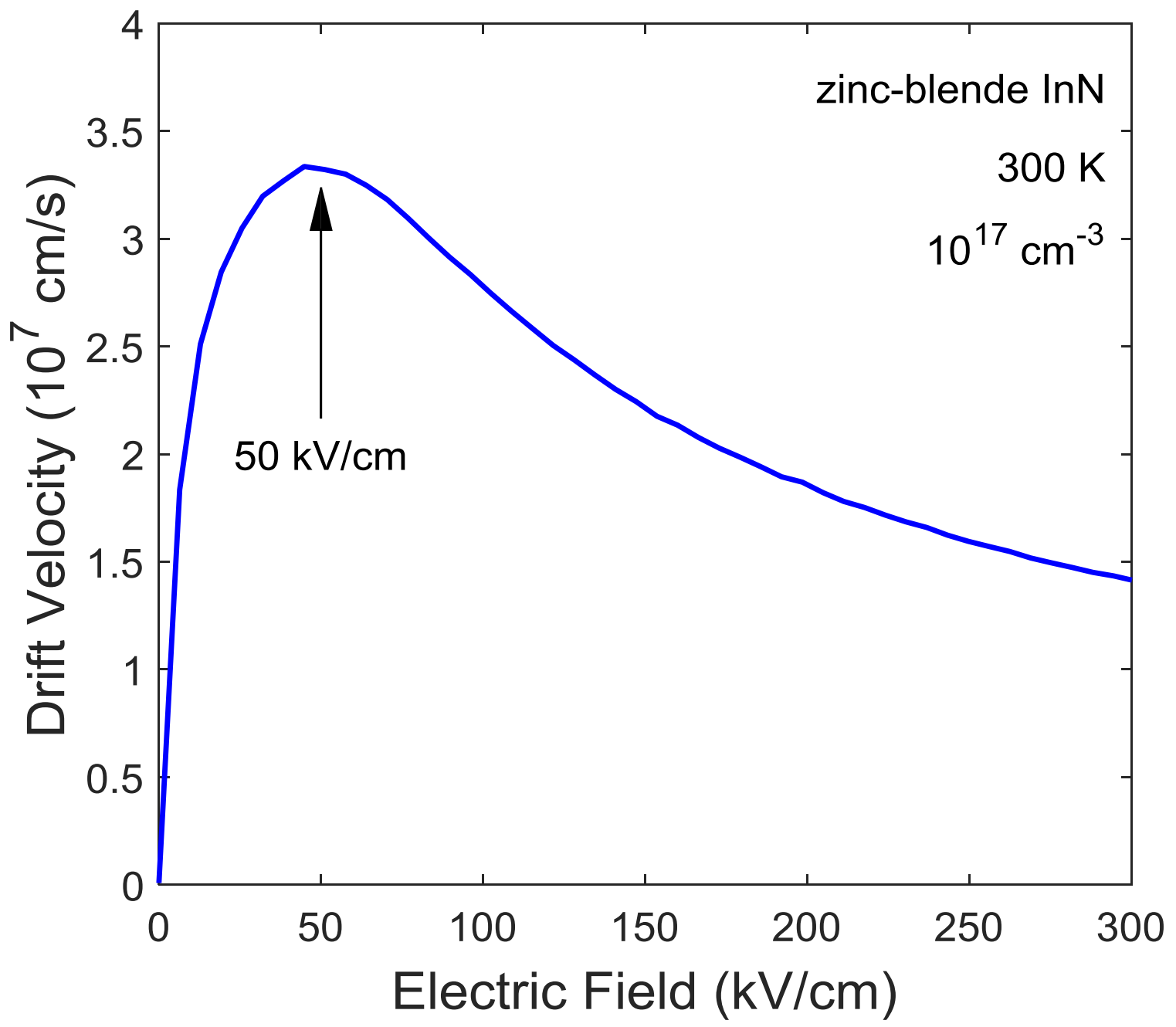

Figure 3.10: The velocity-field characteristic associated with bulk zinc-blende $\ln N$ for the crystal temperature set to $300 \mathrm{~K}$ and the doping concentration set to $10^{17} \mathrm{~cm}^{-3}$. Like many other compound semiconductors, the electron drift velocity reaches a peak, and at higher applied electric field strengths it decreases until it saturates. The peak field, i.e., the applied electric field strength at which the maximum electron drift velocity occurs, $50 \mathrm{kV} / \mathrm{cm}$, is clearly indicated with an arrow. This figure has been modified from () Siddiqua, P. \& O'Leary, S.K. (2018). Electron transport within the wurtzite and zinc-blende phases of gallium nitride and indium nitride, Journal of Materials Science, 29, 3511-3567. Page 3532. Adapted with permission from publisher. The online version of this figure is depicted in color. 


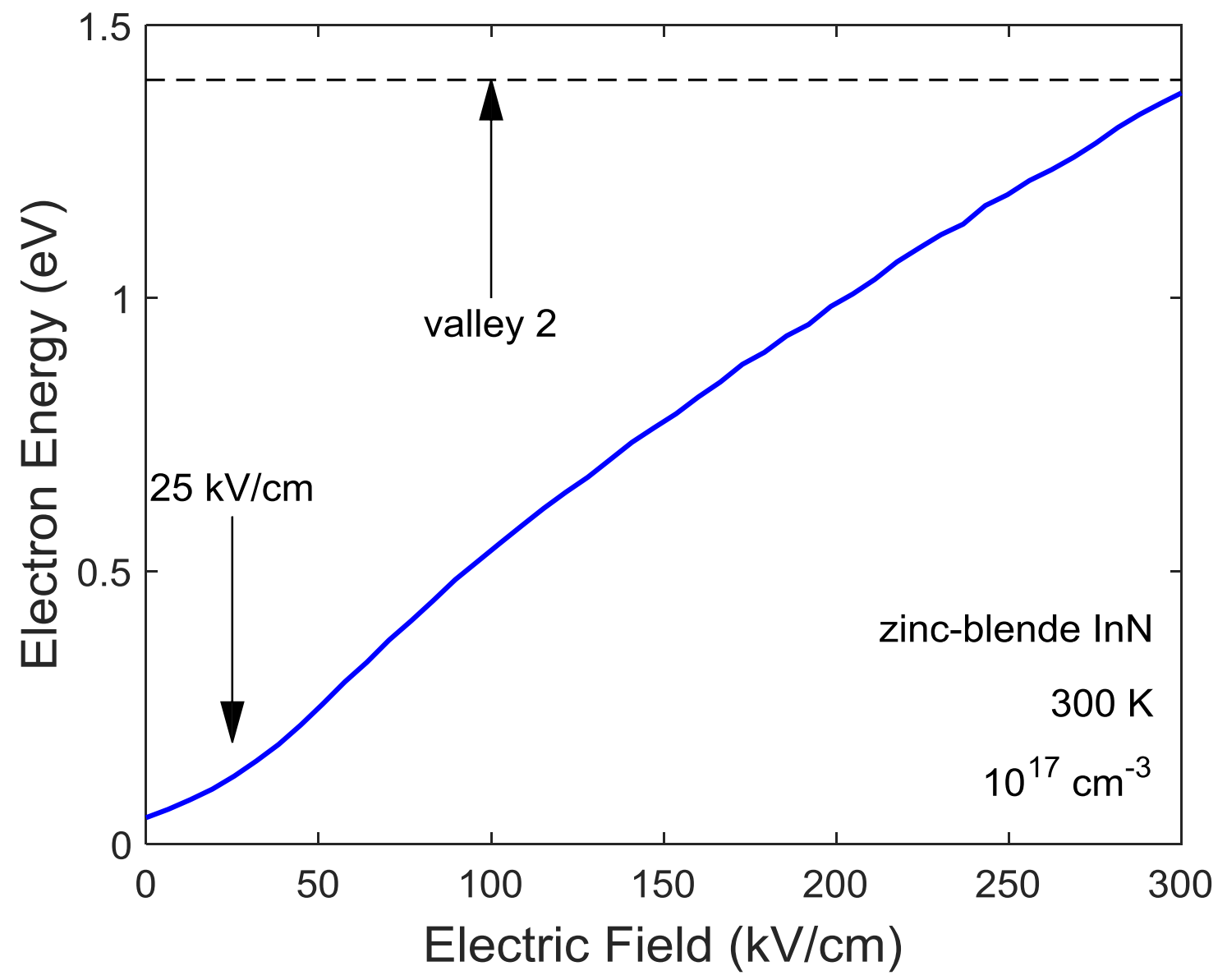

Figure 3.11: The average electron energy as a function of the applied electric field strength for bulk zincblende $\mathrm{InN}$ for the crystal temperature set to $300 \mathrm{~K}$ and the doping concentration set to $10^{17} \mathrm{~cm}^{-3}$. Initially, the average electron energy remains low, only slightly higher than the thermal energy, $3 / 2 k_{B} T$, where $k_{B}$ denotes the Boltzmann constant. At $50 \mathrm{kV} / \mathrm{cm}$, however, the average electron energy increases dramatically. This increase is due to the fact that the polar optical phonon scattering mechanism can no longer absorb all of the energy gained from the applied electric field. The energy minima corresponding to the upper conduction band valley minima is beyond the scale depicted in this figure. This figure has been modified from @ Siddiqua, P. \& O'Leary, S.K. (2018). Electron transport within the wurtzite and zinc-blende phases of gallium nitride and indium nitride, Journal of Materials Science, 29, 3511-3567. Page 3533. Adapted with permission from publisher. The online version of this figure is depicted in color. 


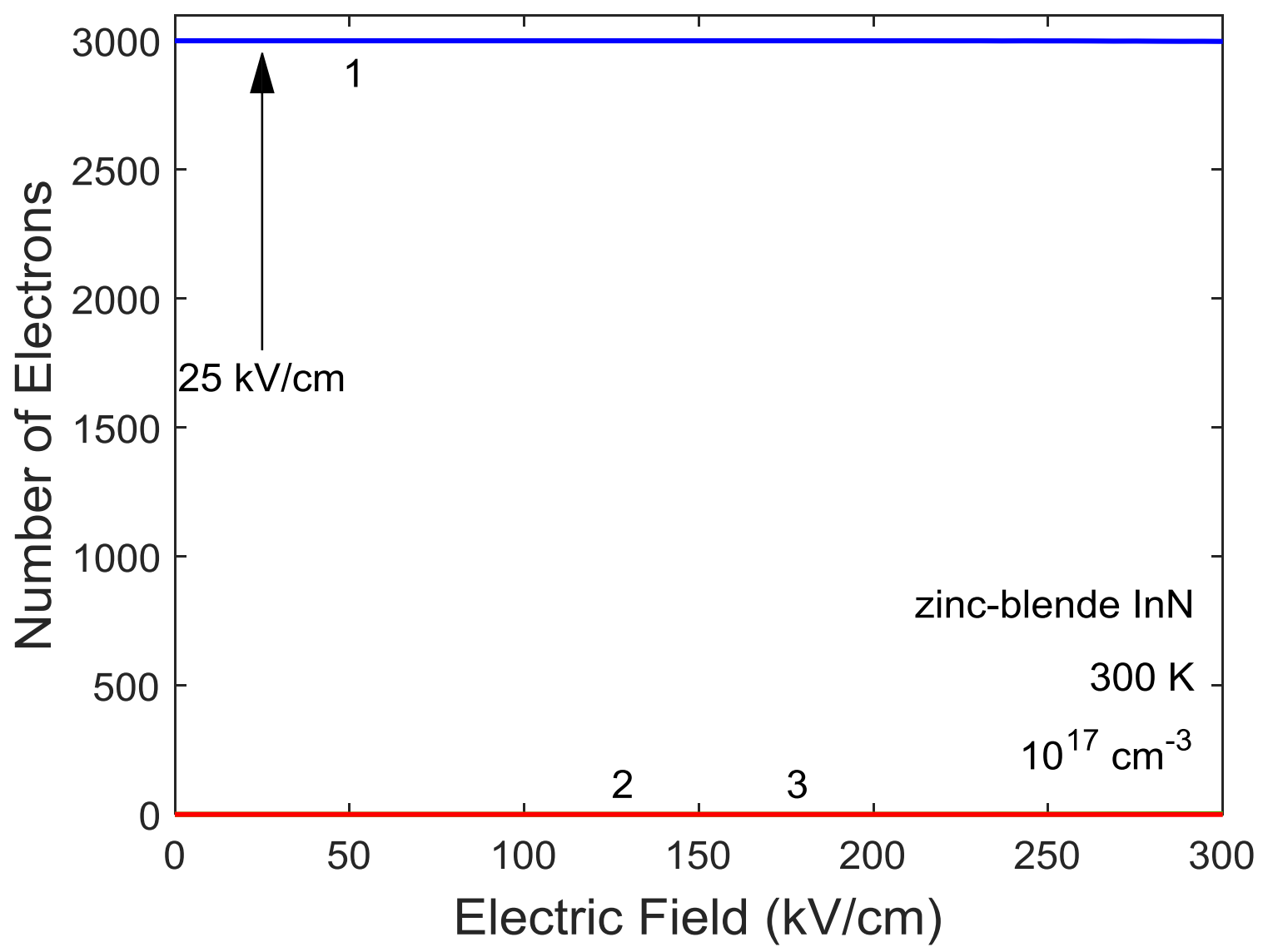

Figure 3.12: The valley occupancy as a function of the applied electric field strength for the case of bulk zinc-blende $\mathrm{InN}$ for the crystal temperature set to $300 \mathrm{~K}$ and the doping concentration set to $10^{17} \mathrm{~cm}^{-3}$. It is observed that no noticeable upper conduction band valley occupancy occurs, even for the very highest applied electric field strengths. This figure has been modified from ( ) Siddiqua, P. \& O’Leary, S.K. (2018). Electron transport within the wurtzite and zinc-blende phases of gallium nitride and indium nitride, Journal of Materials Science, 29, 3511-3567. Page 3533. Adapted with permission from publisher. The online version of this figure is depicted in color. 
ping concentration being set to $10^{17} \mathrm{~cm}^{-3}$. We note that initially the electron drift velocity monotonically increases with the applied electric field strength, reaching a maximum of about 1.6 $\times 10^{7} \mathrm{~cm} / \mathrm{s}$ when the applied electric field strength is around $4 \mathrm{kV} / \mathrm{cm}$. As with the cases of wurtzite and zinc-blende $\mathrm{GaN}$ and $\mathrm{InN}$, a linear regime of electron transport is observed for the case of zinc-blende GaAs, the low-field electron drift mobility, $\mu$, corresponding to the velocity-field characteristic depicted in Figure 3.13 , being about $5400 \mathrm{~cm}^{2} /$ V.s. For applied electric fields strengths in excess of $4 \mathrm{kV} / \mathrm{cm}$, the electron drift velocity decreases in response to further increases in the applied electric field strength, i.e., a region of negative differential mobility is observed, the electron drift velocity eventually saturating at about $1.0 \times 10^{7} \mathrm{~cm} / \mathrm{s}$ for sufficiently high applied electric field strengths.

If we examine the average electron energy as a function of the applied electric field strength, shown in Figure 3.14, we see that there is a sudden increase at around $2 \mathrm{kV} / \mathrm{cm}$; this result is obtained from the same steady-state zinc-blende GaAs Monte Carlo simulation of electron transport as that used to determine Figure 3.13, the crystal temperature being set to 300 $\mathrm{K}$ and the doping concentration being set to $10^{17} \mathrm{~cm}^{-3}$. As with the cases of wurtzite and zincblende GaN and wurtzite InN, beyond a certain critical applied electric field strength, polar optical phonon scattering can no longer remove all of the energy gained from the applied electric field. The average electron energy increases until inter-valley scattering begins and an energy balance is re-established; the energy level of the lowest upper energy conduction band valley minimum corresponding to this material is depicted in Figure 3.14, the second lowest upper energy conduction band valley minimum being beyond the range of energies depicted in Figure 3.14.

In Figure 3.15, we plot the occupancy of the three lowest energy conduction band valleys as a function of the applied electric field strength for the case of zinc-blende GaAs, this result being obtained from the same steady-state zinc-blende GaAs Monte Carlo simulation of electron transport as that used to determine Figures 3.13 and 3.14, the motion of three-thousand electrons 


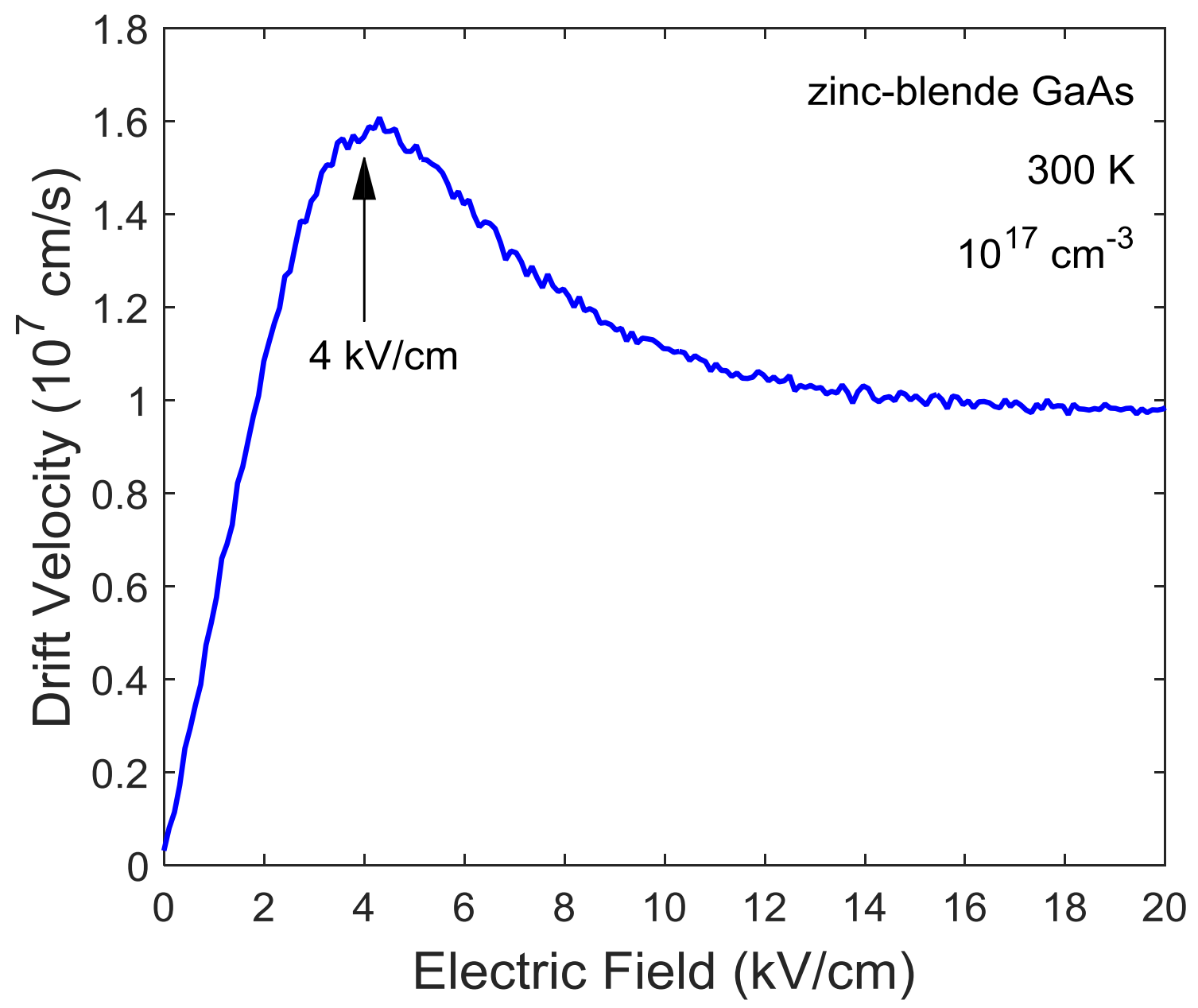

Figure 3.13: The velocity-field characteristic associated with bulk zinc-blende GaAs for the crystal temperature set to $300 \mathrm{~K}$ and the doping concentration set to $10^{17} \mathrm{~cm}^{-3}$. Like many other compound semiconductors, the electron drift velocity reaches a peak, and at higher applied electric field strengths it decreases until it saturates. The peak field, i.e., the applied electric field strength at which the maximum electron drift velocity occurs, $4 \mathrm{kV} / \mathrm{cm}$, is clearly indicated with an arrow. This figure has been modified from (C) Siddiqua, P. \& O'Leary, S.K. (2018). Electron transport within the wurtzite and zinc-blende phases of gallium nitride and indium nitride, Journal of Materials Science, 29, 3511-3567. Page 3534. Adapted with permission from publisher. The online version of this figure is depicted in color. 


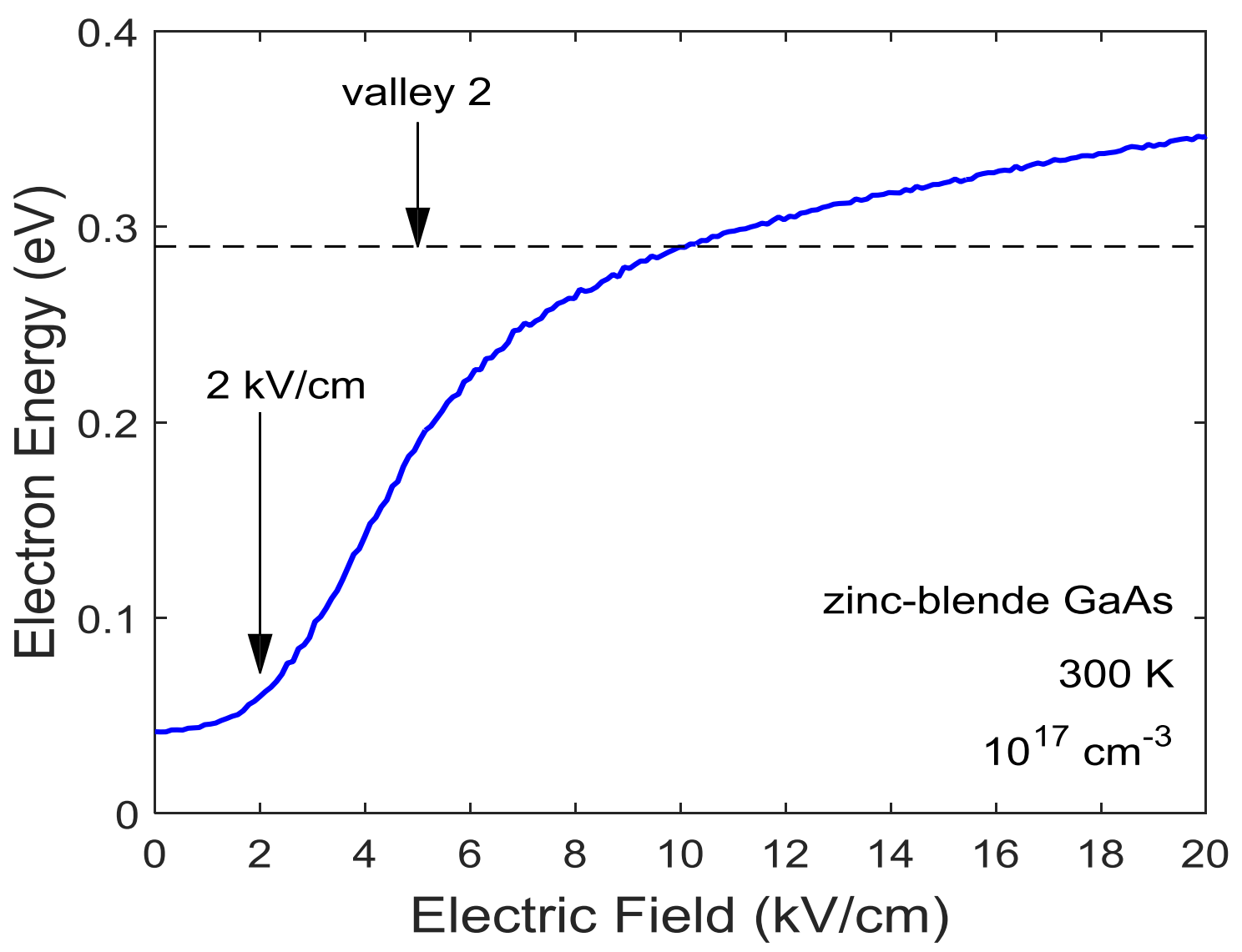

Figure 3.14: The average electron energy as a function of the applied electric field strength for bulk zincblende GaAs for the crystal temperature set to $300 \mathrm{~K}$ and the doping concentration set to $10^{17} \mathrm{~cm}^{-3}$. Initially, the average electron energy remains low, only slightly higher than the thermal energy, $3 / 2 k_{B} T$, where $k_{B}$ denotes the Boltzmann constant. At $2 \mathrm{kV} / \mathrm{cm}$, however, the average electron energy increases dramatically. This increase is due to the fact that the polar optical phonon scattering mechanism can no longer absorb all of the energy gained from the applied electric field. The energy minimum corresponding to the lowest upper conduction band valley minimum is depicted with the dashed line. The other conduction band valley minima are beyond the scale depicted in the figure. This figure has been modified from (c Siddiqua, P. \& O'Leary, S.K. (2018). Electron transport within the wurtzite and zinc-blende phases of gallium nitride and indium nitride, Journal of Materials Science, 29, 3511-3567. Page 3534. Adapted with permission from publisher. The online version of this figure is depicted in color. 


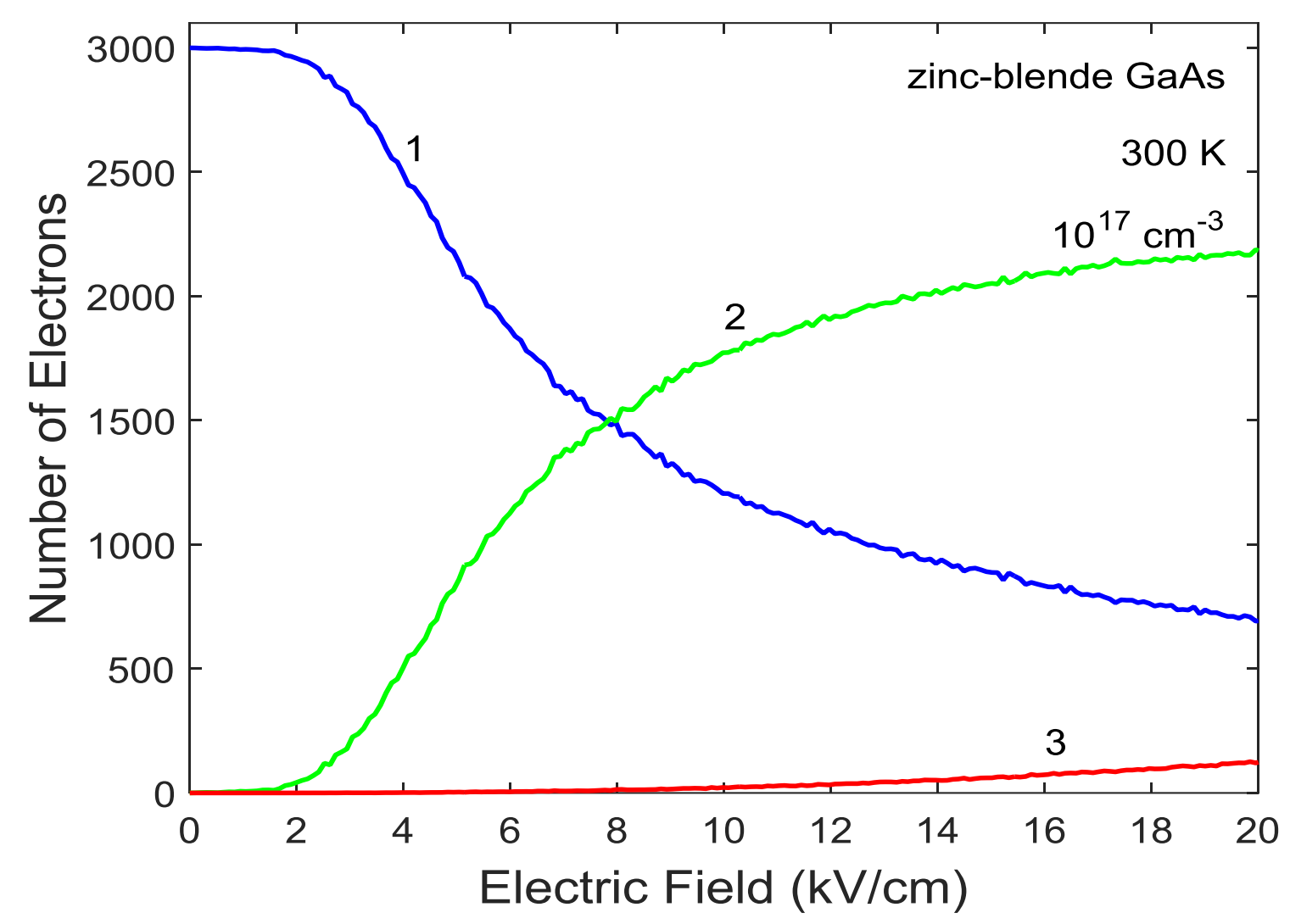

Figure 3.15: The valley occupancy as a function of the applied electric field strength for the case of bulk zinc-blende GaAs for the crystal temperature set to $300 \mathrm{~K}$ and the doping concentration set to $10^{17} \mathrm{~cm}^{-3}$. Soon after the average electron energy increases, i.e., at about $2 \mathrm{kV} / \mathrm{cm}$, electrons begin to transfer to the upper valleys of the conduction band. There were three-thousand electrons employed for this simulation. The valleys are labeled 1,2, and 3, in accordance with their energy minima, i.e., the lowest energy conduction band valley minimum corresponding to valley 1 , the second lowest energy conduction band valley minimum corresponding to valley 2 , the third lowest energy conduction band valley minimum corresponding to valley 3. This figure has been modified from (C Siddiqua, P. \& O'Leary, S.K. (2018). Electron transport within the wurtzite and zinc-blende phases of gallium nitride and indium nitride, Journal of Materials Science, 29, 3511-3567. Page 3535. Adapted with permission from publisher. The online version of this figure is depicted in color. 
being considered for this steady-state electron transport analysis, the crystal temperature being set to $300 \mathrm{~K}$ and the doping concentration being set to $10^{17} \mathrm{~cm}^{-3}$. This result is similar to that found for the cases of wurtzite and zinc-blende $\mathrm{GaN}$ and wurtzite InN, i.e., a lot of upper energy conduction band valley occupancy occurs as the applied electric field strength is increased.

\subsection{Steady-state electron transport: a comparison of the results associated with the wurtzite and zinc-blende phases of GaN and InN with those associated with zinc-blende GaAs}

In Figure $3.16 \mathrm{a}$, we contrast the velocity-field characteristics associated with the semiconductors under consideration in this analysis, i.e., the wurtzite and zinc-blende phases of GaN and InN. For all cases, we have set the crystal temperature to $300 \mathrm{~K}$ and the doping concentration to $10^{17} \mathrm{~cm}^{-3}$, the material and band structural parameters being as specified in Tables 2.1 and 2.2, respectively, i.e., these results are the same as those presented in Figures 3.1, 3.4, 3.7, and 3.10, for the cases of wurtzite GaN, zinc-blende GaN, wurtzite InN, and zincblende $\ln N$, respectively. We see that each of these wide energy gap compound semiconductors achieves a peak in its velocity-field characteristic. Wurtzite $\ln N$ achieves the highest steady-state peak electron drift velocity, about $5.6 \times 10^{7} \mathrm{~cm} / \mathrm{s}$ at an applied electric field strength of around 30 $\mathrm{kV} / \mathrm{cm}$. This contrasts with the case of wurtzite $\mathrm{GaN}, 2.9 \times 10^{7} \mathrm{~cm} / \mathrm{s}$ at $140 \mathrm{kV} / \mathrm{cm}$, the case of zinc-blende GaN, $3.3 \times 10^{7} \mathrm{~cm} / \mathrm{s}$ at $110 \mathrm{kV} / \mathrm{cm}$, and that of zinc-blende $\operatorname{lnN}, 3.3 \times 10^{7} \mathrm{~cm} / \mathrm{s}$ at 50 $\mathrm{kV} / \mathrm{cm}$.

For the case of zinc-blende GaAs, the peak electron drift velocity, $1.6 \times 10^{7} \mathrm{~cm} / \mathrm{s}$, occurs at a much lower applied electric field strength than that for the other compound semiconductors considered in this analysis, i.e., only $4 \mathrm{kV} / \mathrm{cm}$. In order to contrast the velocity-field characteristics associated with the wurtzite and zinc-blende phases of GaN and $\ln N$ with that associated with zinc-blende GaAs, it is instructive to recast these steady-state results onto a logarithmic scale, the resultant plot being depicted in Figure 3.16b. This velocity-field plot renders obvious the utility 
of the wide energy gap semiconductor class for high-field device applications, i.e., even though their low-field mobility may be lower than that associated with their more conventional counterparts, such as GaAs, at higher electric field strengths, the velocity of the electrons within these semiconductors is quite large.

For the purposes of quick comparison, it is useful to plot the key metrics associated with these steady-state results for the different materials considered in this analysis. The peak and saturation electron drift velocities associated with these materials are depicted in Figure 3.16c. The peak electric field strengths, i.e., the electric field strengths at which these peak steady-state electron drift velocities occur, are depicted in Figure 3.16d.

\subsection{The sensitivity of the velocity-field characteristics associated with wurtzite GaN, zinc-blende GaN, wurtzite InN, zinc-blende InN, and zinc-blende GaAs to variations in the crystal temperature}

The sensitivity of the velocity-field characteristics to variations in the crystal temperature is now explored. In Figures. 3.17a, 3.18a, 3.19a, and 3.20a, the velocity-field characteristics associated with wurtzite $\mathrm{GaN}$, zinc-blende $\mathrm{GaN}$, wurtzite $\operatorname{lnN}$, and zinc-blende $\operatorname{lnN}$, respectively, are presented for a number of different crystal temperatures; crystal temperatures between 100 to $900 \mathrm{~K}$, in increments of $200 \mathrm{~K}$, are considered in this analysis. The upper limit, $900 \mathrm{~K}$, is chosen as this corresponds to some of the highest operating temperature which may be reasonably expected for AlGaN/GaN power devices; a recent analysis has shown that some of the III-V nitride semiconductors can tolerate temperatures which actually approach $1200 \mathrm{~K}$, although for actual device applications, some margin of safety would be desired [158]. It is noted that crystal temperature variations do indeed play a significant role in shaping these velocity-field characteristics. Focusing on the peak electron drift velocity itself, for the specific case of wurtzite 


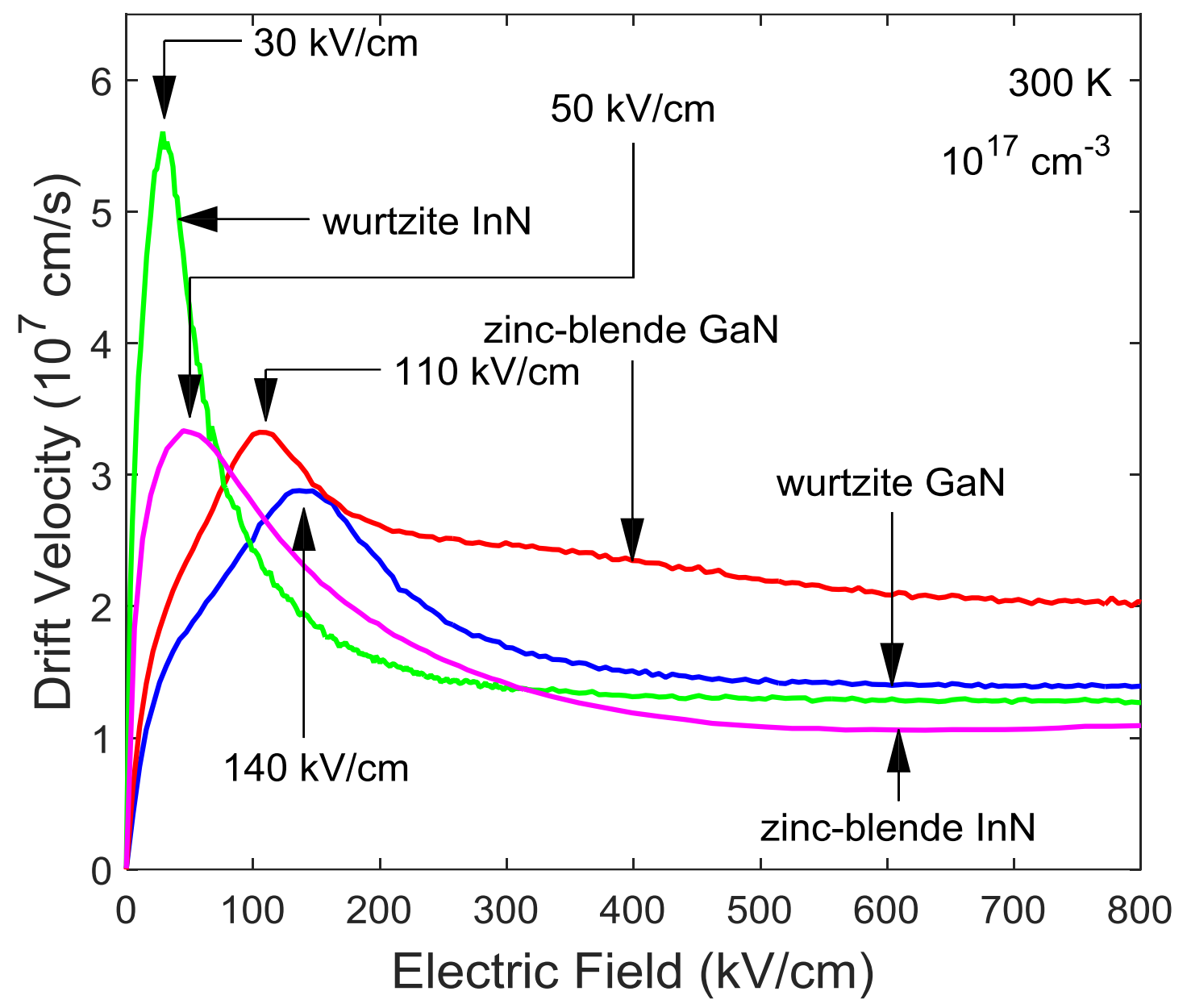

Figure 3.16.a: A comparison of the velocity-field characteristics associated with the wurtzite and zincblende phases of GaN and InN. The arrows correspond to the peak fields, i.e., the applied electric field strengths at which the peaks in the velocity-field characteristics occur, for each material considered. The crystal temperature is set to $300 \mathrm{~K}$ and the doping concentration is set to $10^{17} \mathrm{~cm}^{-3}$ for all cases. This plot is depicted on a linear scale. This figure has been modified from @ Siddiqua, P. \& O'Leary, S.K. (2018). Electron transport within the wurtzite and zinc-blende phases of gallium nitride and indium nitride, Journal of Materials Science, 29, 3511-3567. Page 3536. Adapted with permission from publisher. The online version of this figure is depicted in color. 


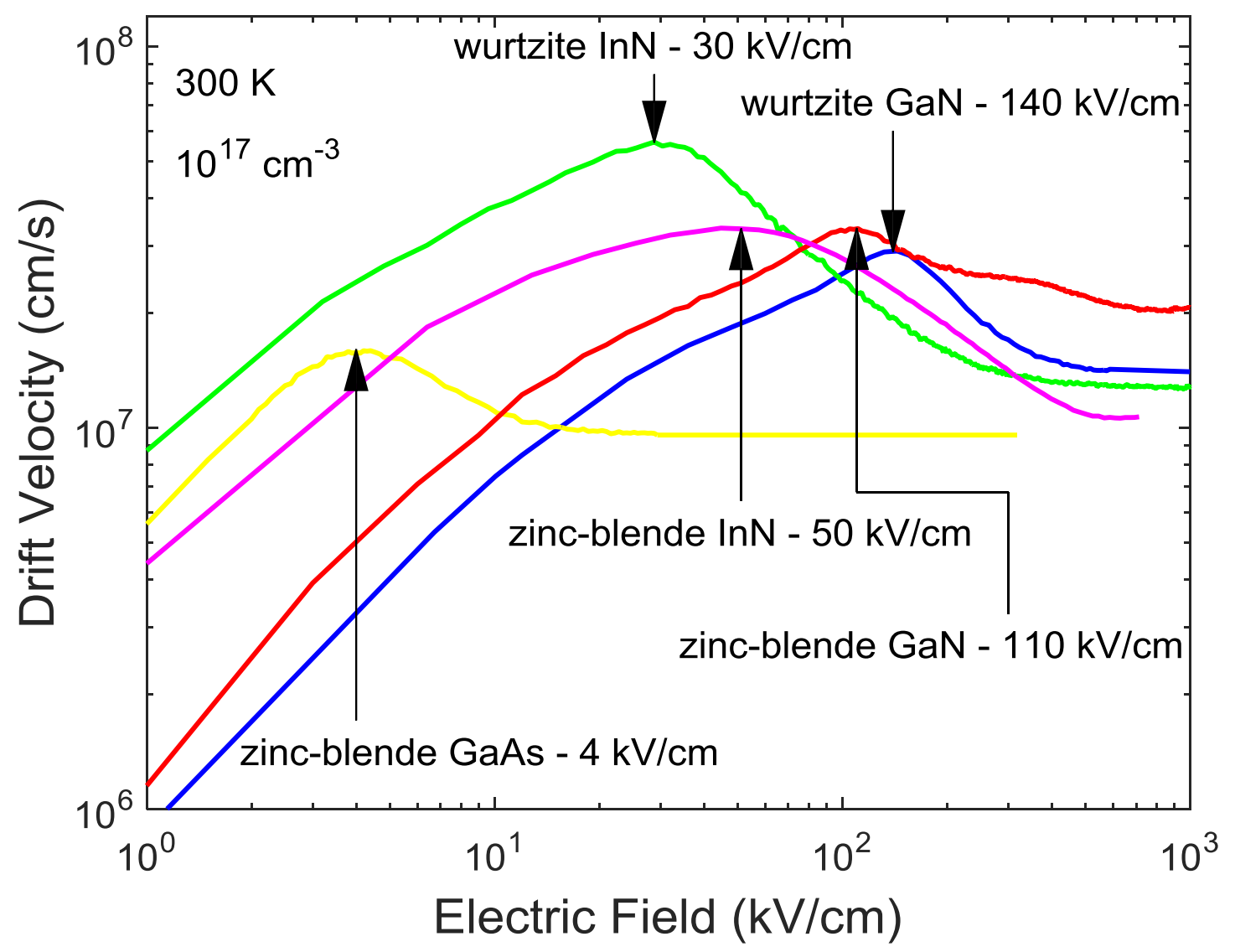

Figure 3.16.b: A comparison of the velocity-field characteristics associated with the wurtzite and zincblende phases of $\mathrm{GaN}$ and InN, with that associated with zinc-blende GaAs. The arrows correspond to the peak fields, i.e., the applied electric field strengths at which the peaks in the velocity-field characteristics occur, for each material considered. The crystal temperature is set to $300 \mathrm{~K}$ and the doping concentration is set to $10^{17} \mathrm{~cm}^{-3}$ for all cases. This plot is depicted on a logarithmic scale. This figure has been modified from @ ( Siddiqua, P. \& O'Leary, S.K. (2018). Electron transport within the wurtzite and zinc-blende phases of gallium nitride and indium nitride, Journal of Materials Science, 29, 3511-3567. Page 3536. Adapted with permission from publisher. The online version of this figure is depicted in color. 


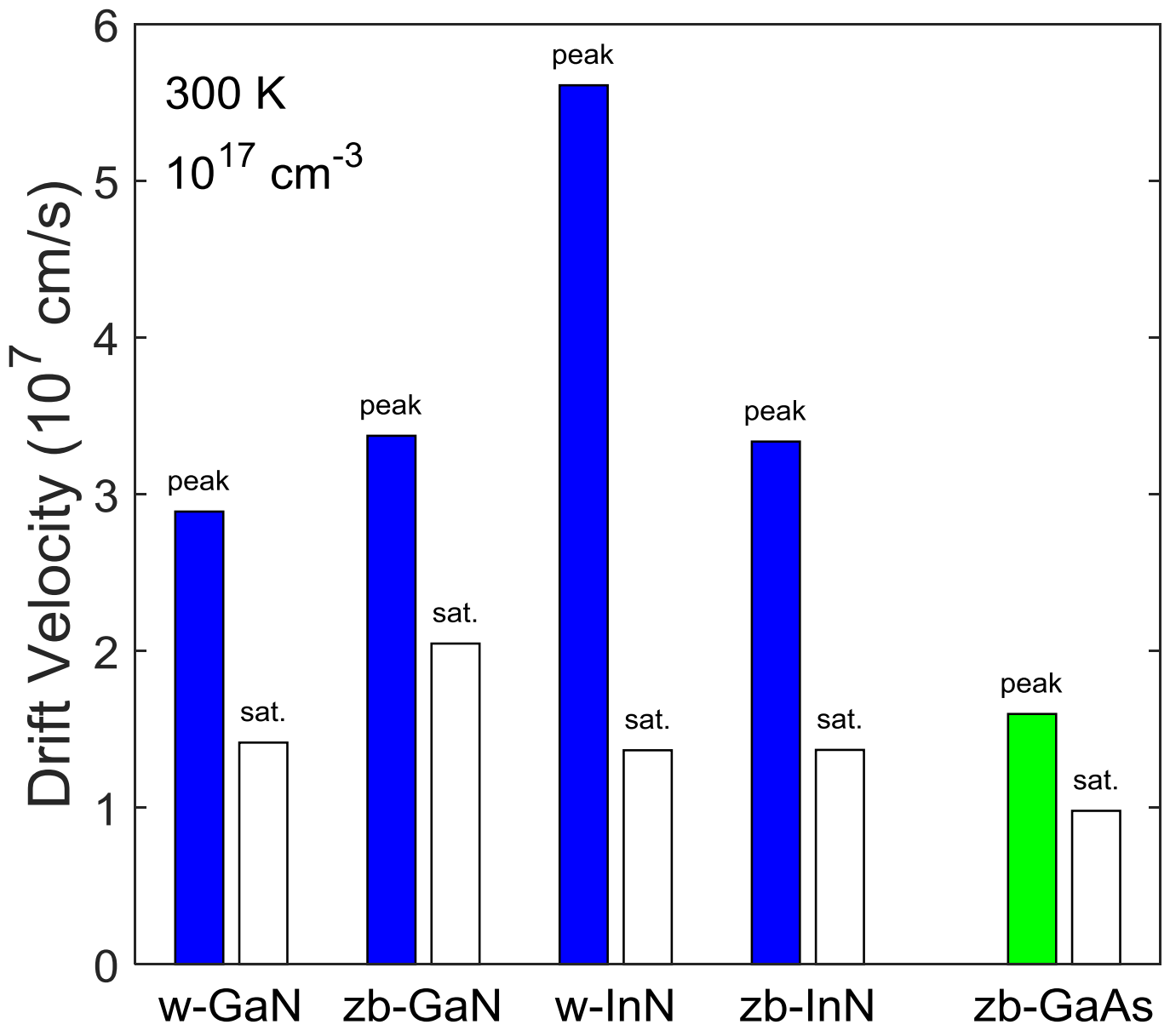

Figure 3.16.c: A comparison of the peak and saturation electron drift velocities associated with the wurtzite and zinc-blende phases $\mathrm{GaN}$ and InN, with that associated with zinc-blende GaAs. The crystal temperature is set to $300 \mathrm{~K}$ and the doping concentration is set to $10^{17} \mathrm{~cm}^{-3}$ for all cases. This figure has been modified from (c) Siddiqua, P. \& O'Leary, S.K. (2018). Electron transport within the wurtzite and zinc-blende phases of gallium nitride and indium nitride, Journal of Materials Science, 29, 3511-3567. Page 3536. Adapted with permission from publisher. The online version of this figure is depicted in color. 


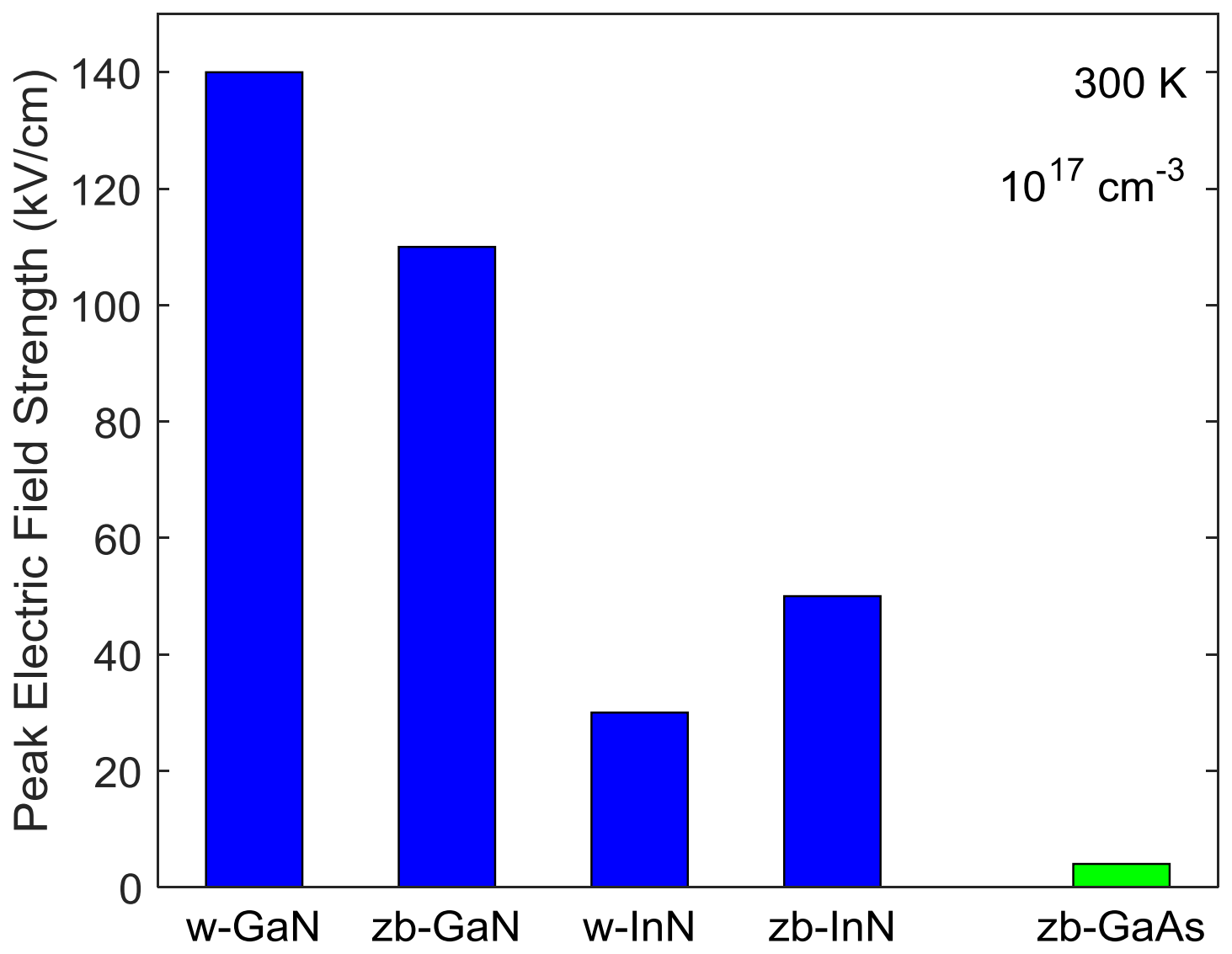

Figure 3.16.d: A comparison of the peak electric fields, i.e., the electric field strengths at which the peak electron drift velocities occur, associated with the wurtzite and zinc-blende phases of GaN and InN, with that associated with zinc-blende GaAs. The crystal temperature is set to $300 \mathrm{~K}$ and the doping concentration is set to $10^{17} \mathrm{~cm}^{-3}$ for all cases. This figure has been modified from (C) Siddiqua, P. \& O'Leary, S.K. (2018). Electron transport within the wurtzite and zinc-blende phases of gallium nitride and indium nitride, Journal of Materials Science, 29, 3511-3567. Page 3536. Adapted with permission from publisher. The online version of this figure is depicted in color. 
GaN, this velocity decreases from around $3.1 \times 10^{7} \mathrm{~cm} / \mathrm{s}$ at $100 \mathrm{~K}$ to about $2.0 \times 10^{7} \mathrm{~cm} / \mathrm{s}$ at 900 K. The corresponding peak field strengths, i.e., the electric field strengths at which these peaks occur, are also found to vary with the crystal temperature, from around $130 \mathrm{kV} / \mathrm{cm}$ at $100 \mathrm{~K}$ to about $180 \mathrm{kV} / \mathrm{cm}$ at $900 \mathrm{~K}$. Similar results are found for the other materials considered and the other electron transport metrics.

To highlight the difference between the wide energy gap compound semiconductors, GaN and InN, with more conventional III-V compound semiconductors, such as GaAs, Monte Carlo simulations of the electron transport within zinc-blende GaAs have also been performed under the same conditions as the other materials. Figure 3.21 a shows the results of these simulations. Clearly, the velocity-field characteristics associated with the wide energy gap compound semiconductors, GaN and InN, are less sensitive to variations in the crystal temperature than those associated with zinc-blende GaAs. A combination of scattering rates and occupancy issues account for the differences in behaviour, as has been explained by O'Leary et al. [115].

To quantify these dependencies further, the peak and saturation electron drift velocities associated with wurtzite $\mathrm{GaN}$, zinc-blende $\mathrm{GaN}$, wurtzite $\operatorname{lnN}$, and zinc-blende $\ln N$ are plotted as functions of the crystal temperature in Figures 3.17b, 3.18b, 3.19b, and 3.20b, respectively, these results being determined from our steady-state Monte Carlo simulations of the electron transport within these materials. Analogous results, corresponding to zinc-blende GaAs, are depicted in Figure 3.21b. For all materials, it is found that all of these electron transport metrics diminish as the crystal temperature is increased. As may be seen through an inspection of Figures 3.17, 3.18, $3.19,3.20$ and 3.21 , inclusive, the peak and saturation electron drift velocities do not drop as much in $\mathrm{GaN}$ and InN as they do in GaAs in response to increases in the crystal temperature. This property will undoubtedly have an impact on high-power device performance. 


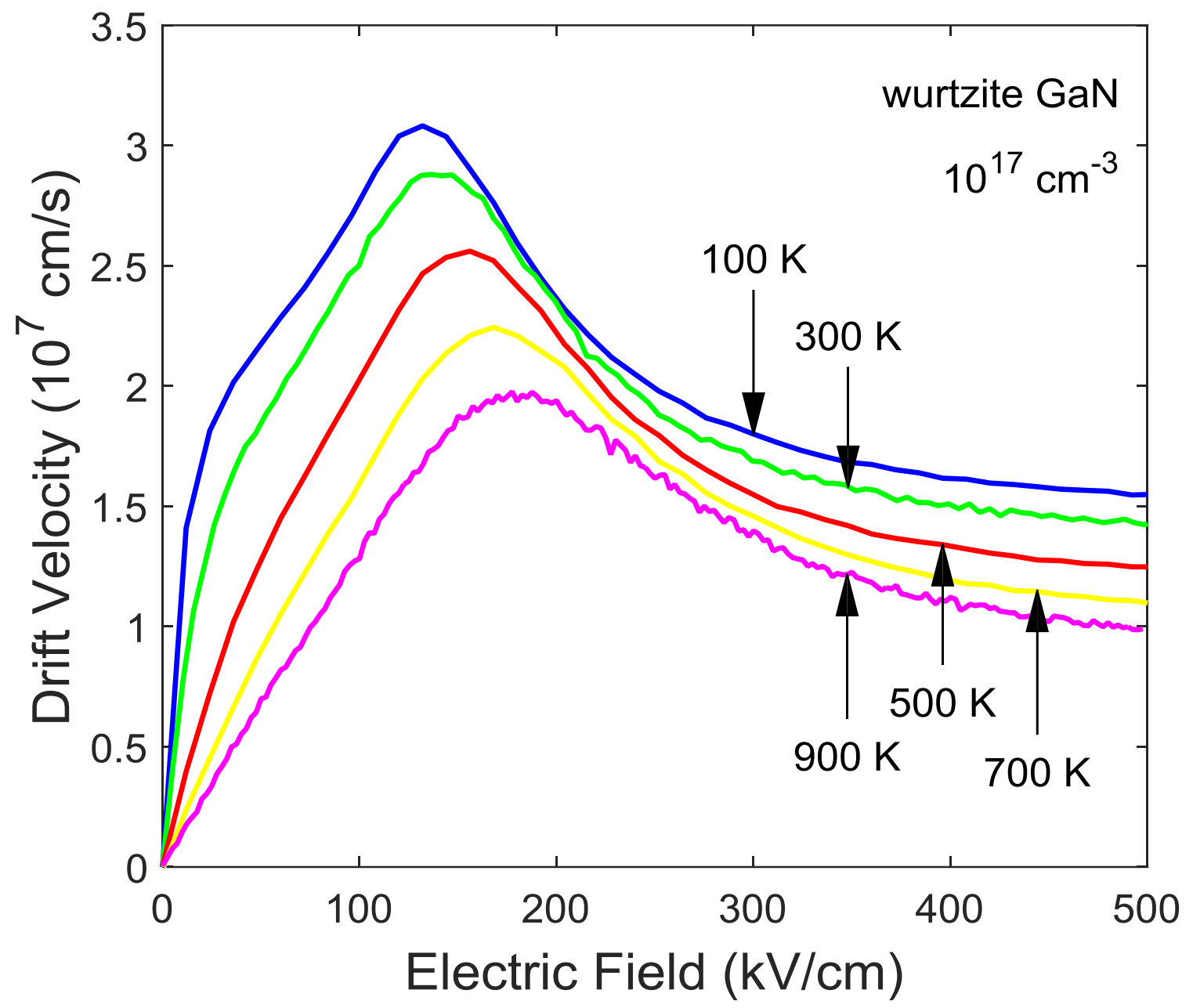

Figure 3.17.a: The velocity-field characteristics associated with bulk wurtzite GaN for various crystal temperatures. For all cases, I have assumed a doping concentration of $10^{17} \mathrm{~cm}^{-3}$. This figure has been modified from @ Siddiqua, P. \& O'Leary, S.K. (2018). Electron transport within the wurtzite and zinc-blende phases of gallium nitride and indium nitride, Journal of Materials Science, 29, 3511-3567. Page 3537. Adapted with permission from publisher. The online version of this figure is depicted in color. 


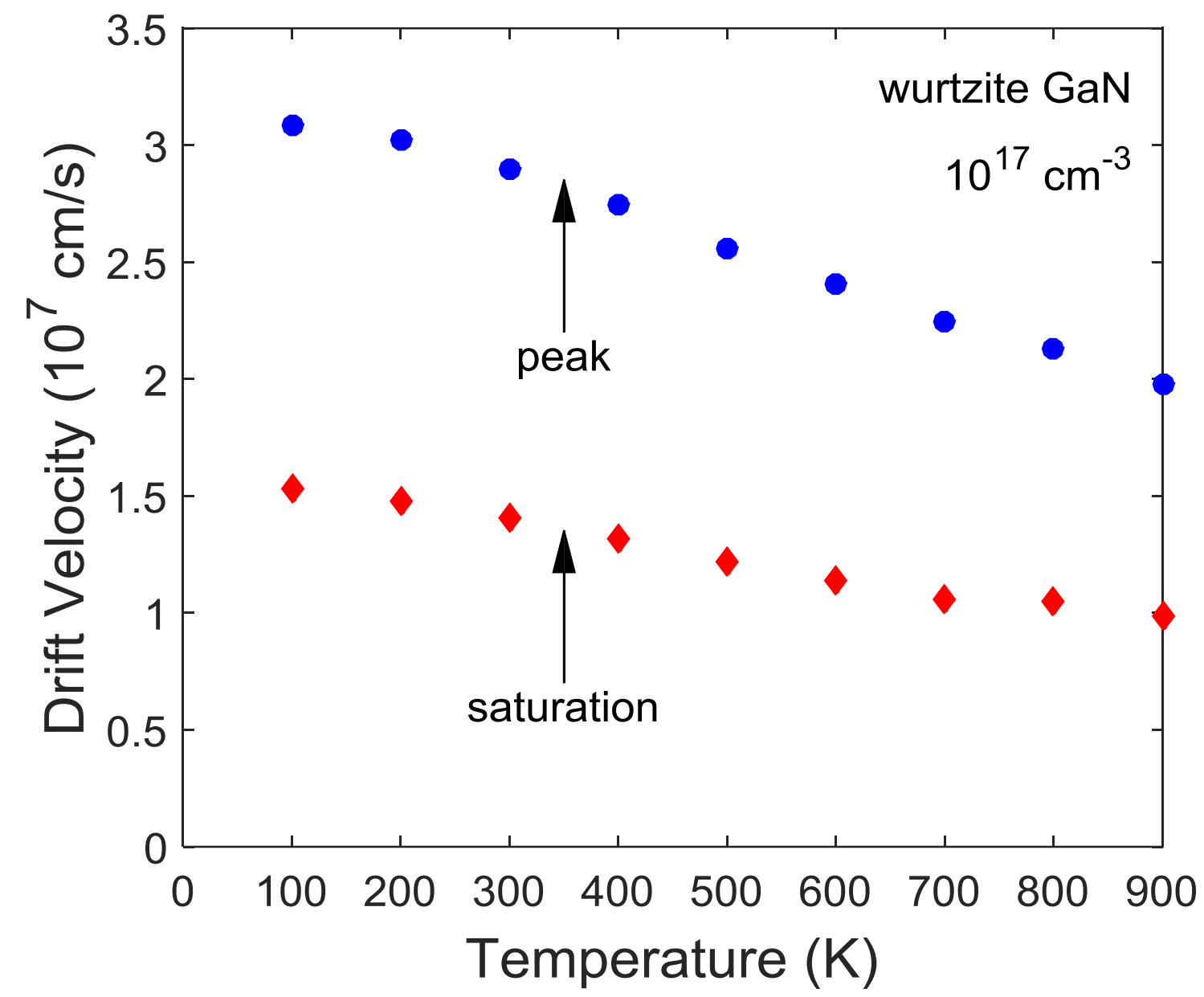

Figure 3.17.b: The peak and saturation electron drift velocities associated with bulk wurtzite GaN as a function of the crystal temperature. These results are determined from the results of Monte Carlo simulations of electron transport within bulk wurtzite GaN. For all cases, I have assumed a doping concentration of $10^{17} \mathrm{~cm}^{-3}$. This figure has been modified from @ C Siddiqua, P. \& O'Leary, S.K. (2018). Electron transport within the wurtzite and zinc-blende phases of gallium nitride and indium nitride, Journal of Materials Science, 29, 3511-3567. Page 3537. Adapted with permission from publisher. The online version of this figure is depicted in color. 


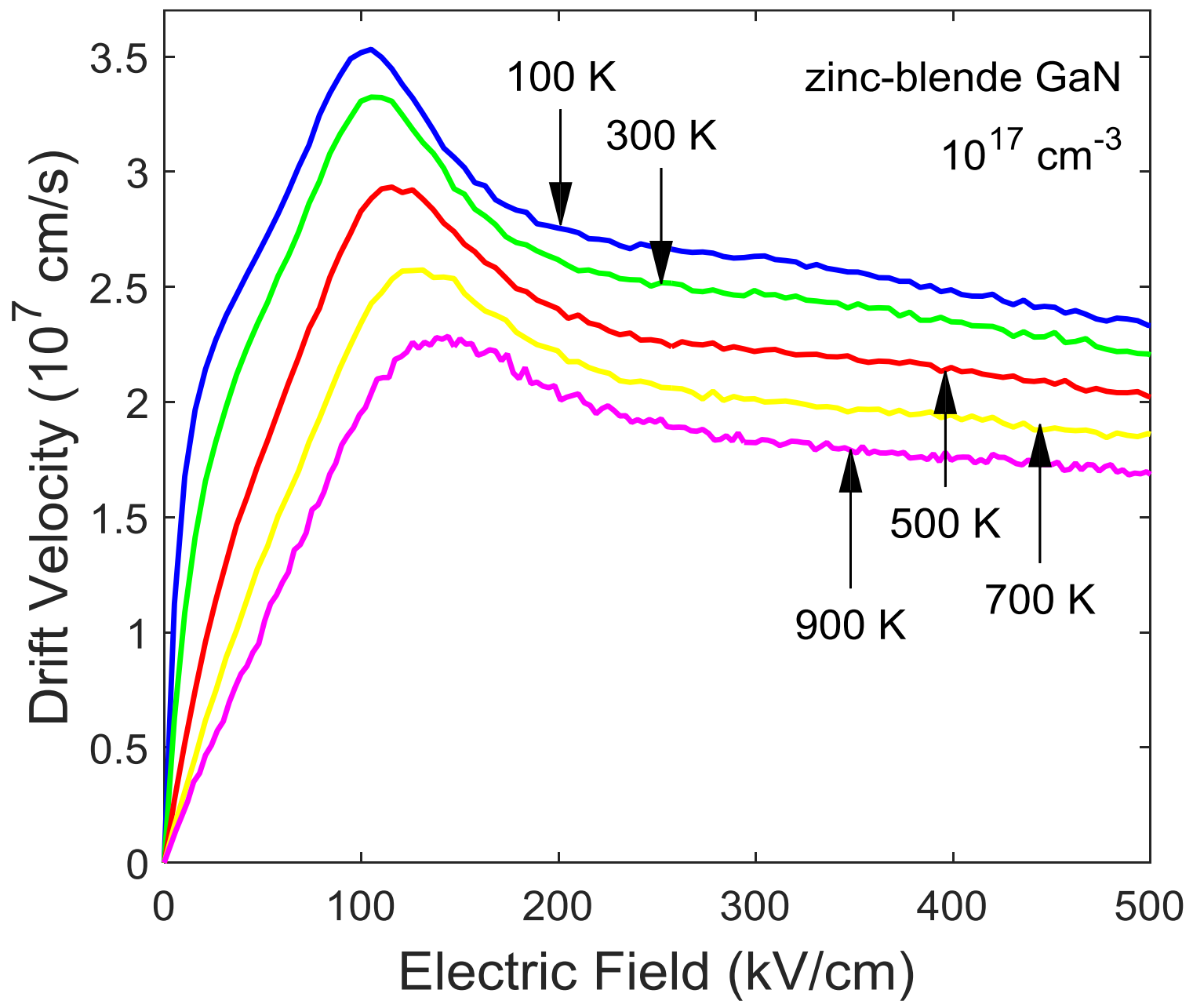

Figure 3.18.a: The velocity-field characteristics associated with bulk zinc-blende GaN for various crystal temperatures. For all cases, I have assumed a doping concentration of $10^{17} \mathrm{~cm}^{-3}$. This figure has been modified from (C) Siddiqua, P. \& O'Leary, S.K. (2018). Electron transport within the wurtzite and zinc-blende phases of gallium nitride and indium nitride, Journal of Materials Science, 29, 3511-3567. Page 3537. Adapted with permission from publisher. The online version of this figure is depicted in color. 


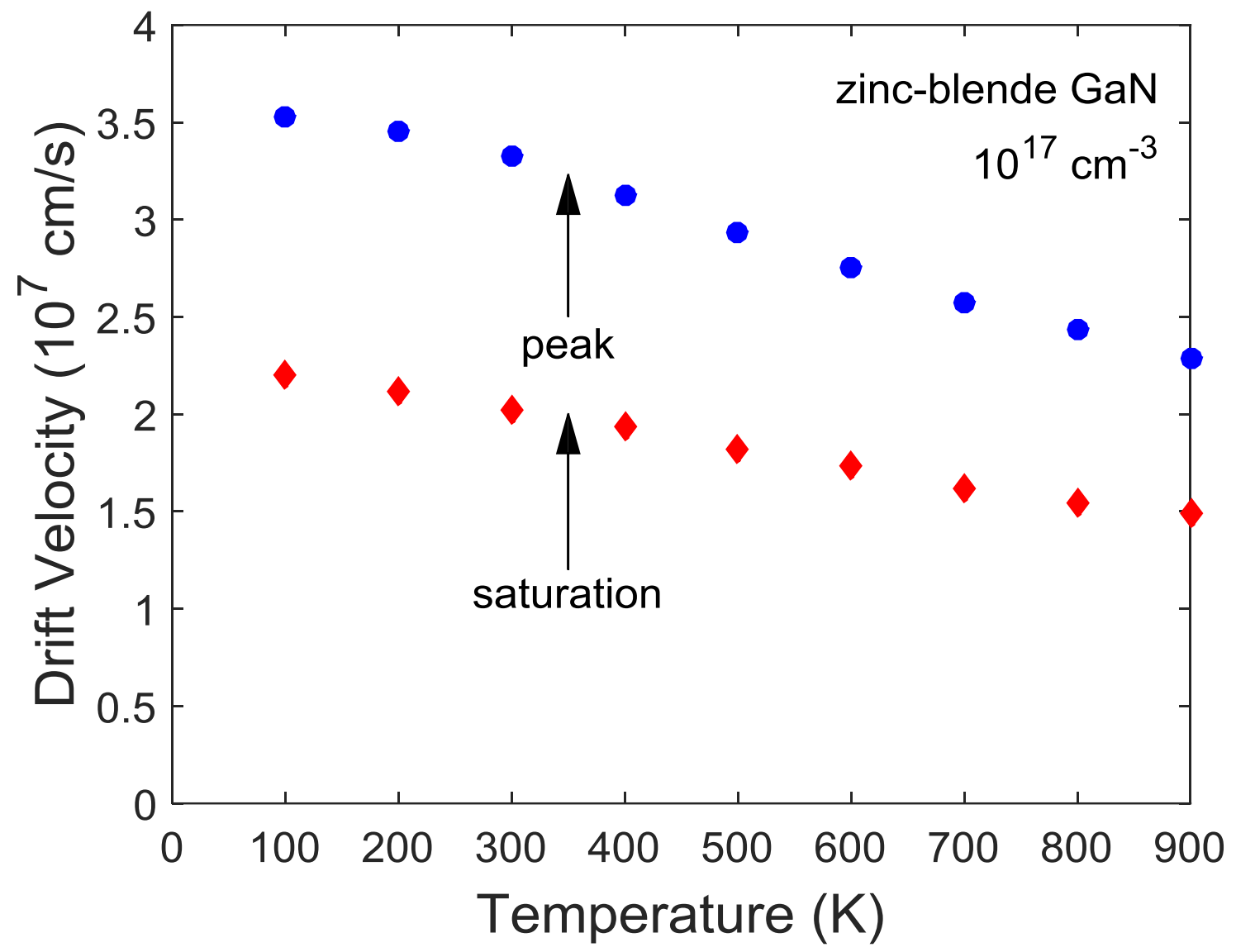

Figure 3.18.b: The peak and saturation electron drift velocities associated with bulk zinc-blende GaN as a function of the crystal temperature. These results are determined from the results of Monte Carlo simulations of electron transport within bulk zinc-blende GaN. For all cases, I have assumed a doping concentration of $10^{17} \mathrm{~cm}^{-3}$. This figure has been modified from (c) Siddiqua, P. \& O'Leary, S.K. (2018). Electron transport within the wurtzite and zinc-blende phases of gallium nitride and indium nitride, Journal of Materials Science, 29, 3511-3567. Page 3537. Adapted with permission from publisher. The online version of this figure is depicted in color. 


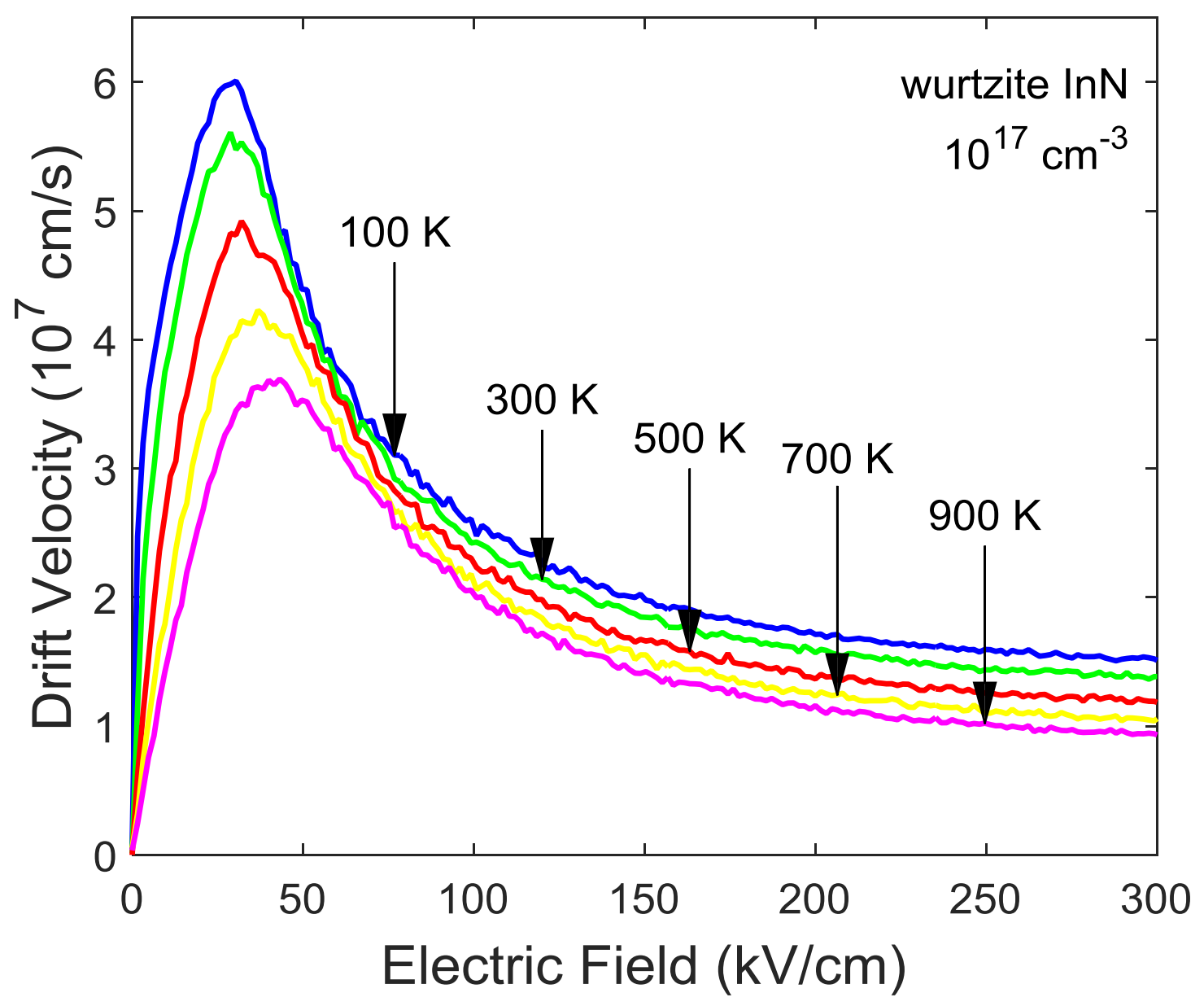

Figure 3.19.a: The velocity-field characteristics associated with bulk wurtzite $\ln N$ for various crystal temperatures. For all cases, I have assumed a doping concentration of $10^{17} \mathrm{~cm}^{-3}$. This figure has been modified from @ Siddiqua, P. \& O'Leary, S.K. (2018). Electron transport within the wurtzite and zinc-blende phases of gallium nitride and indium nitride, Journal of Materials Science, 29, 3511-3567. Page 3538. Adapted with permission from publisher. The online version of this figure is depicted in color. 


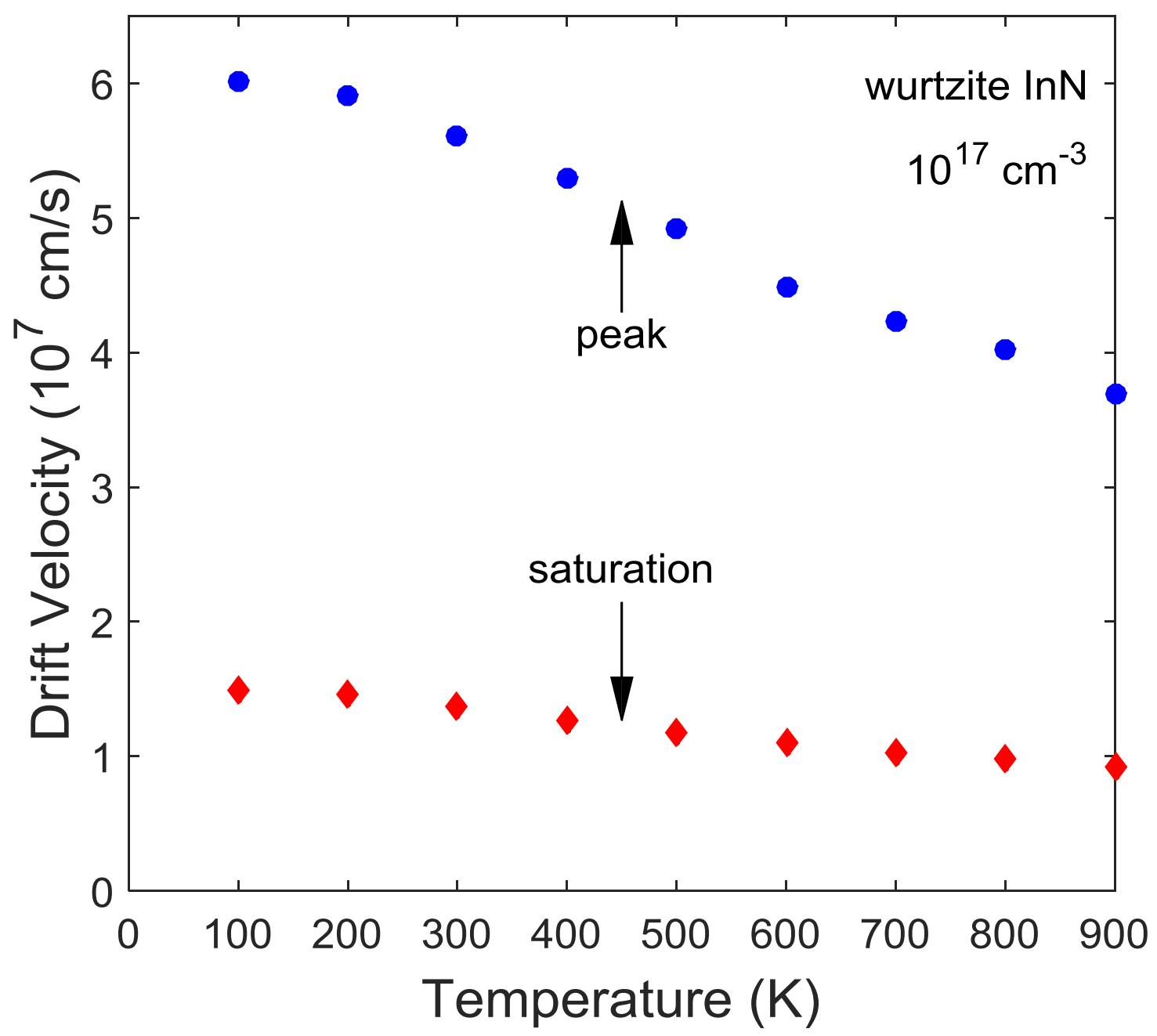

Figure 3.19.b: The peak and saturation electron drift velocities associated with bulk wurtzite $\operatorname{lnN}$ as a function of the crystal temperature. These results are determined from the results of Monte Carlo simulations of electron transport within bulk wurtzite InN. For all cases, I have assumed a doping concentration of $10^{17} \mathrm{~cm}^{-3}$. This figure has been modified from ( ) Siddiqua, P. \& O'Leary, S.K. (2018). Electron transport within the wurtzite and zinc-blende phases of gallium nitride and indium nitride, Journal of Materials Science, 29, 3511-3567. Page 3538. Adapted with permission from publisher. The online version of this figure is depicted in color. 


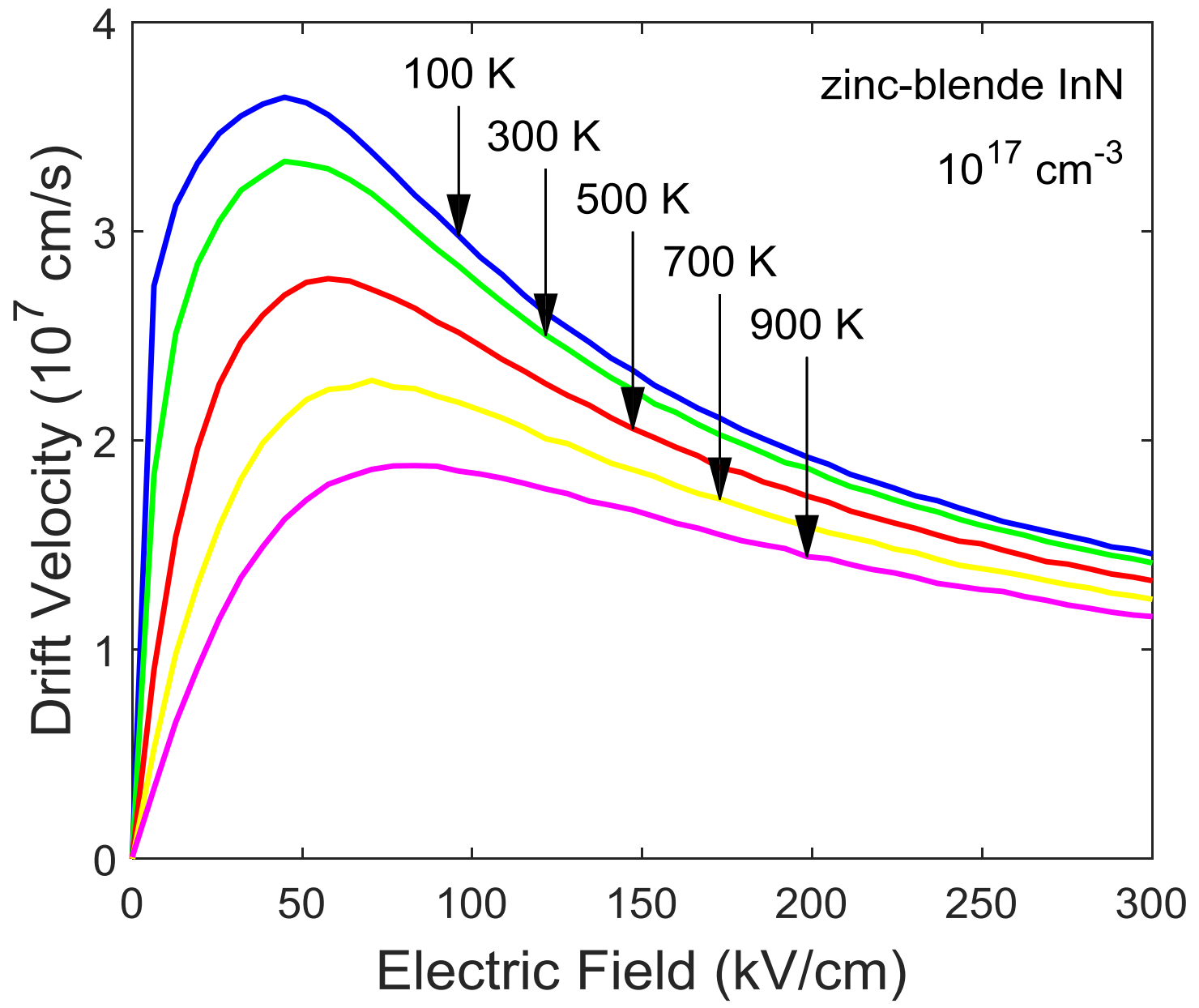

Figure 3.20.a: The velocity-field characteristics associated with bulk zinc-blende $\ln N$ for various crystal temperatures. For all cases, I have assumed a doping concentration of $10^{17} \mathrm{~cm}^{-3}$. This figure has been modified from @ ( Siddiqua, P. \& O'Leary, S.K. (2018). Electron transport within the wurtzite and zinc-blende phases of gallium nitride and indium nitride, Journal of Materials Science, 29, 3511-3567. Page 3538. Adapted with permission from publisher. The online version of this figure is depicted in color. 


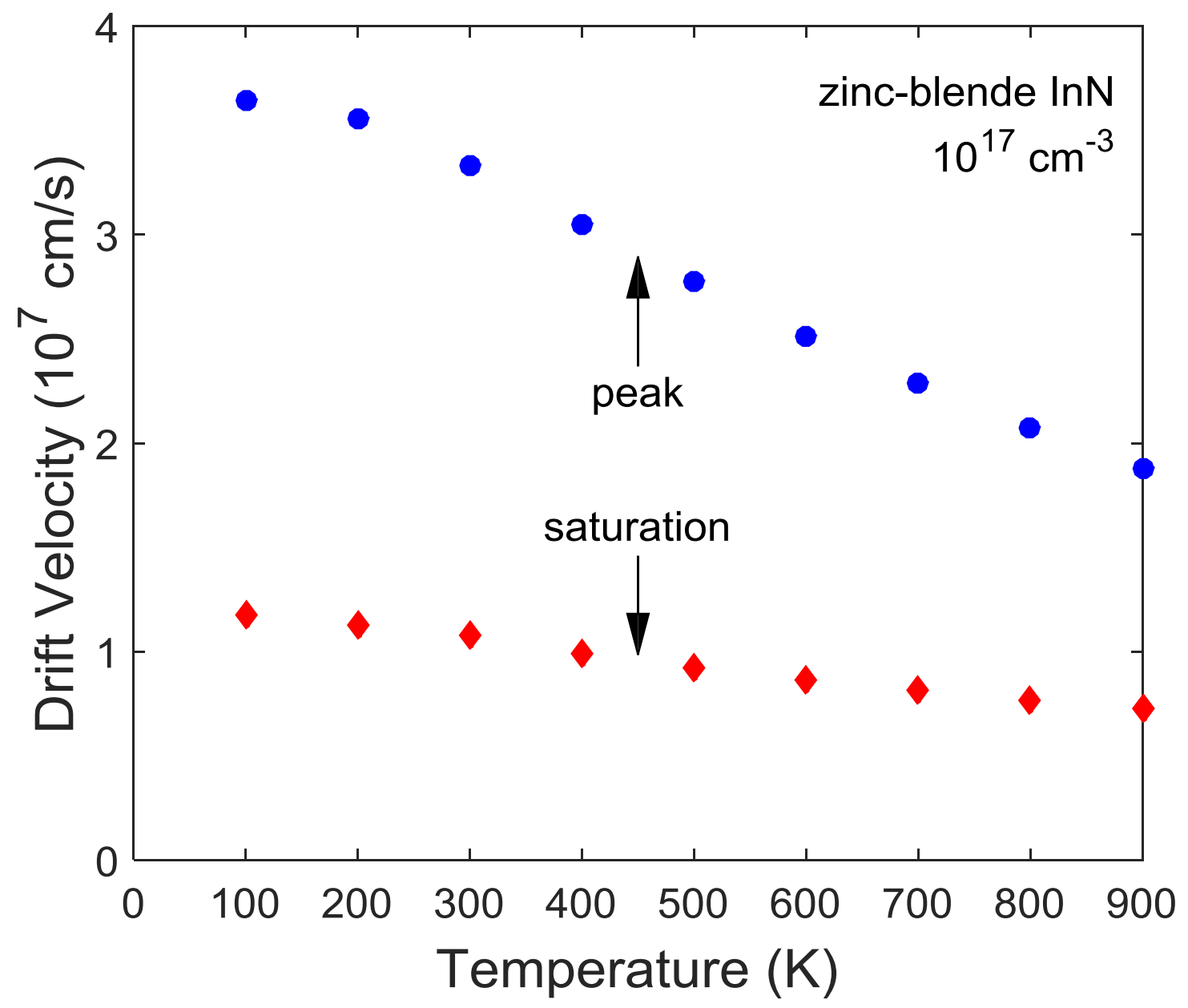

Figure 3.20.b: The peak and saturation electron drift velocities associated with bulk zinc-blende $\ln N$ as a function of the crystal temperature. These results are determined from the results of Monte Carlo simulations of electron transport within bulk zinc-blende InN. For all cases, I have assumed a doping concentration of $10^{17} \mathrm{~cm}^{-3}$. This figure has been modified from (C) Siddiqua, P. \& O'Leary, S.K. (2018). Electron transport within the wurtzite and zinc-blende phases of gallium nitride and indium nitride, Journal of Materials Science, 29, 3511-3567. Page 3538. Adapted with permission from publisher. The online version of this figure is depicted in color. 


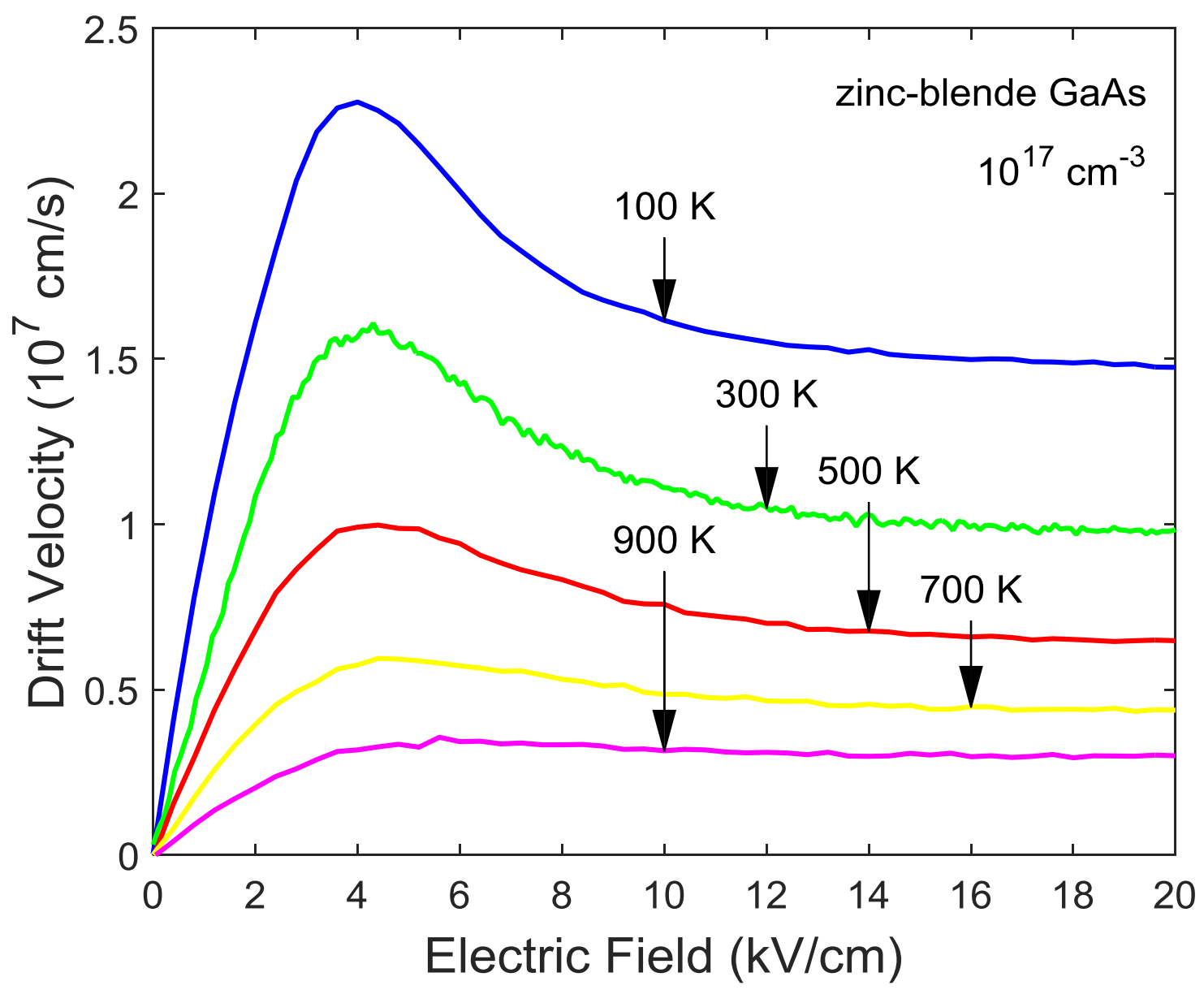

Figure 3.21.a: The velocity-field characteristics associated with bulk zinc-blende GaAs for various crystal temperatures. For all cases, I have assumed a doping concentration of $10^{17} \mathrm{~cm}^{-3}$. This figure has been modified from @ Siddiqua, P. \& O'Leary, S.K. (2018). Electron transport within the wurtzite and zinc-blende phases of gallium nitride and indium nitride, Journal of Materials Science, 29, 3511-3567. Page 3539. Adapted with permission from publisher. The online version of this figure is depicted in color. 


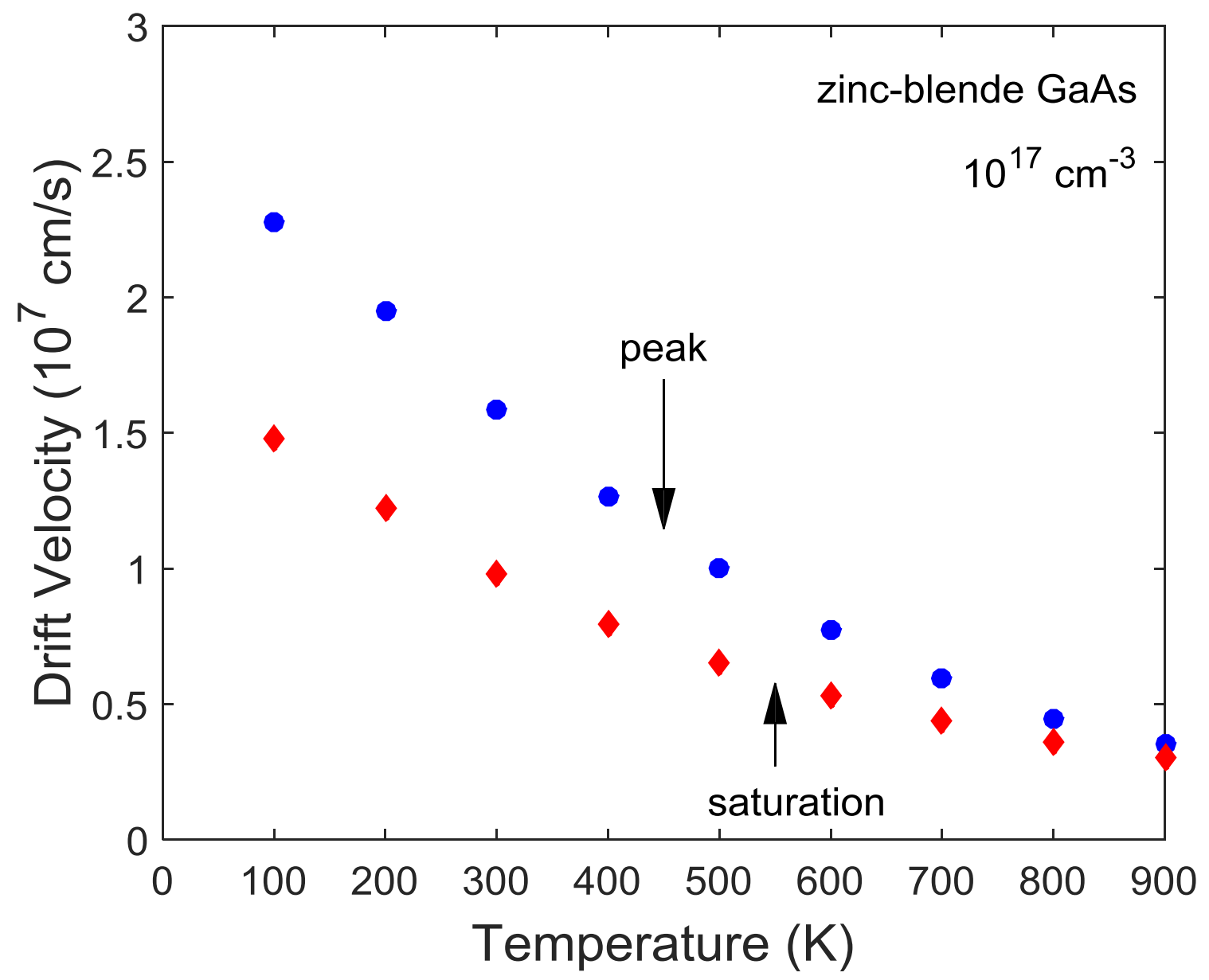

Figure 3.21.b: The peak and saturation electron drift velocities associated with bulk zinc-blende GaAs as a function of the crystal temperature. These results are determined from the results of Monte Carlo simulations of electron transport within bulk zinc-blende GaAs. For all cases, I have assumed a doping concentration of $10^{17} \mathrm{~cm}^{-3}$. This figure has been modified from (c) Siddiqua, P. \& O'Leary, S.K. (2018). Electron transport within the wurtzite and zinc-blende phases of gallium nitride and indium nitride, Journal of Materials Science, 29, 3511-3567. Page 3539. Adapted with permission from publisher. The online version of this figure is depicted in color. 


\subsection{The sensitivity of the velocity-field characteristics associated with wurtzite GaN, zinc-blende GaN, wurtzite InN, zinc-blende InN, and zinc-blende GaAs to variations in the doping concentrations}

The doping concentration is a parameter which can be readily controlled in the fabrication of a semiconductor device. Understanding the effect of the doping concentration on the resultant electron transport characteristics is important. In Figures. 3.22a, 3.23a, 3.24a, and 3.25a, the velocity-field characteristics associated with wurtzite $\mathrm{GaN}$, zinc-blende $\mathrm{GaN}$, wurtzite InN, and zinc-blende InN, respectively, are presented for a number of different doping concentration levels; doping concentrations between $10^{16}$ and $10^{19} \mathrm{~cm}^{-3}$ are considered, in decade increments; the $10^{16}$ $\mathrm{cm}^{-3}$ result is imperceptibly distinct from the $10^{17} \mathrm{~cm}^{-3}$ result for the specific case of wurtzite GaN, and thus, is not shown for this particular case. It is noted that variations in the doping concentration do indeed play a significant role in shaping these velocity-field characteristics.

Focusing on the peak electron drift velocity itself, for the specific case of wurtzite GaN, it is found that this velocity decreases from around $2.9 \times 10^{7} \mathrm{~cm} / \mathrm{s}$ at $10^{17} \mathrm{~cm}^{-3}$ to about $2.0 \times 10^{7}$ $\mathrm{cm} / \mathrm{s}$ at $10^{19} \mathrm{~cm}^{-3}$. The corresponding peak field strengths, i.e., the applied electric field strengths at which the peak in the electron drift velocity occurs, are also found to vary with the doping concentration, albeit slightly, i.e., from around $140 \mathrm{kV} / \mathrm{cm}$ at $10^{17} \mathrm{~cm}^{-3}$ to about $130 \mathrm{kV} / \mathrm{cm}$ at $10^{19}$ $\mathrm{cm}^{-3}$. Similar results are found for the other materials considered and for the other electron transport metrics.

To highlight the difference between the wide energy gap compound semiconductors, GaN and InN, with more conventional III-V compound semiconductors, such as GaAs, Monte Carlo simulations of the electron transport within zinc-blende GaAs have also been performed under the same conditions as the other materials; for the case of zinc-blende GaAs, as the $10^{16} \mathrm{~cm}^{-3}$ result is visually distinct from the $10^{17} \mathrm{~cm}^{-3}$ result, the $10^{16} \mathrm{~cm}^{-3}$ result is also depicted. Figure 
3.26.a shows the results of these simulations. Clearly, the velocity-field characteristics associated with the wide energy gap compound semiconductors, GaN and InN, are less sensitive to variations in the doping concentration than those associated with zinc-blende GaAs; in fact, for the case of $10^{19} \mathrm{~cm}^{-3}$ doping, the peak in the velocity-field characteristic associated with zincblende GaAs completely disappears, the velocity-field characteristic associated with zinc-blende GaAs monotonically increasing with the applied electric field strength until saturation is achieved for this particular case. A combination of scattering rates and occupancy issues account for the differences in behaviour, as has been explained by O'Leary et al. [115].

To quantify these dependencies further, the peak and saturation electron drift velocities associated with wurtzite $\mathrm{GaN}$, zinc-blende $\mathrm{GaN}$, wurtzite $\operatorname{lnN}$, zinc-blende $\operatorname{lnN}$, and zinc-blende GaAs are plotted as functions of the doping concentration in Figures 3.22.b, 3.23.b, 3.24.b, 3.25.b, and 3.26.b, respectively, these results being determined from our steady-state Monte Carlo simulations of the electron transport within these materials. For all the materials considered in this analysis, it is found that all of these electron transport metrics diminish as the doping concentration is increased. As may be seen through an inspection of Figures 3.22 through 3.26, inclusive, the peak and saturation electron drift velocities do not drop as much in GaN and InN as they do in GaAs in response to increases in the doping concentration. Clearly, this points to another advantage of the wide energy gap semiconductors.

\subsection{Transient electron transport}

Steady-state electron transport is the dominant electron transport mechanism in devices with larger dimensions. For devices with smaller dimensions, however, transient electron transport must also be considered when evaluating device performance. Ruch [159] demonstrated, for both Si and GaAs, that the transient electron drift velocity may exceed the corr- 


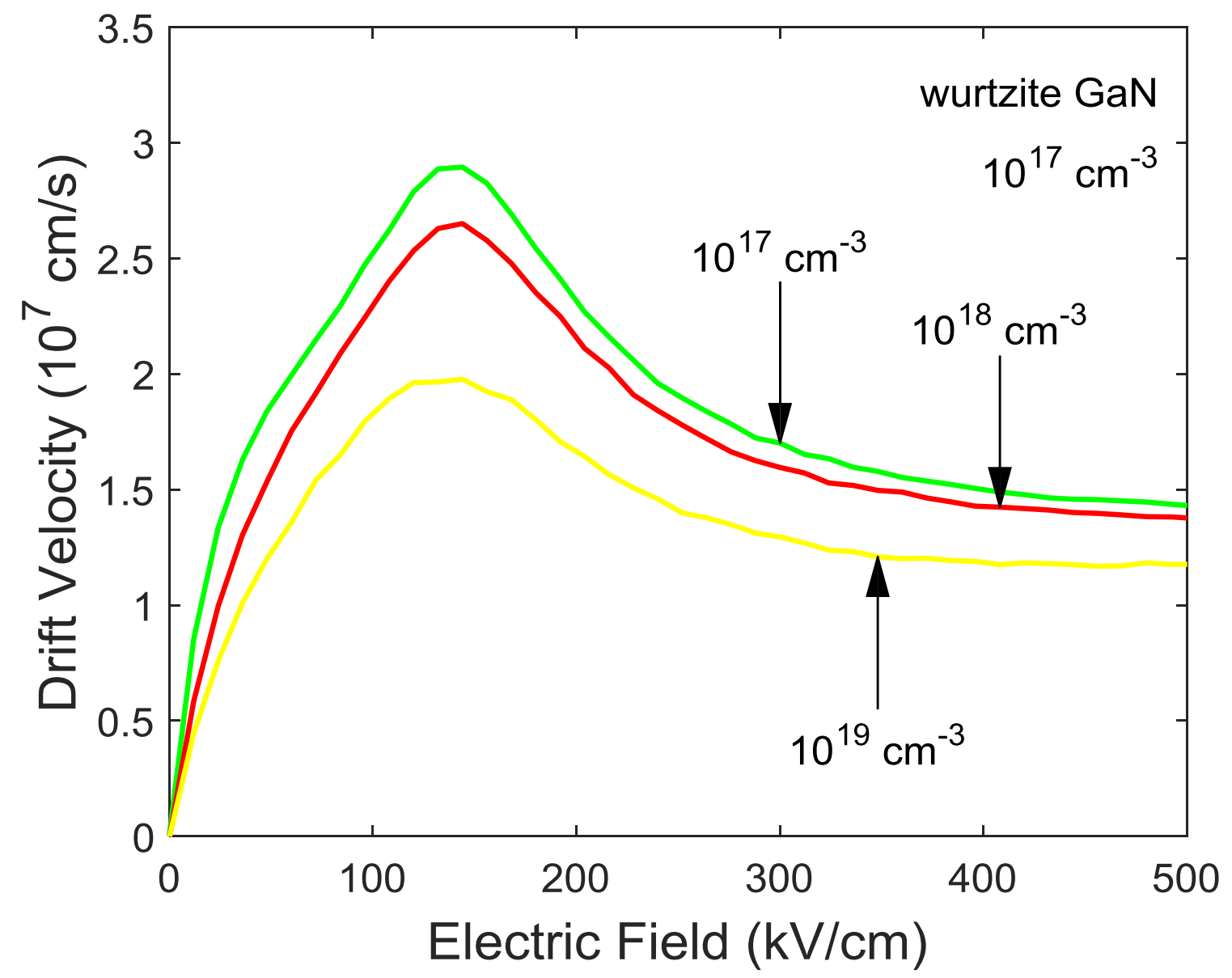

Figure 3.22.a: The velocity-field characteristics associated with bulk wurtzite GaN for various doping concentrations. For all cases, I have assumed a crystal temperature of $300 \mathrm{~K}$. The $10^{16} \mathrm{~cm}^{-3}$ doping concentration case is not shown in this plot as it is essentially indistinguishable from the $10^{17} \mathrm{~cm}^{-3}$ case for this particular material. This figure has been modified from @ Siddiqua, P. \& O'Leary, S.K. (2018). Electron transport within the wurtzite and zinc-blende phases of gallium nitride and indium nitride, Journal of Materials Science, 29, 3511-3567. Page 3539. Adapted with permission from publisher. The online version of this figure is depicted in color. 


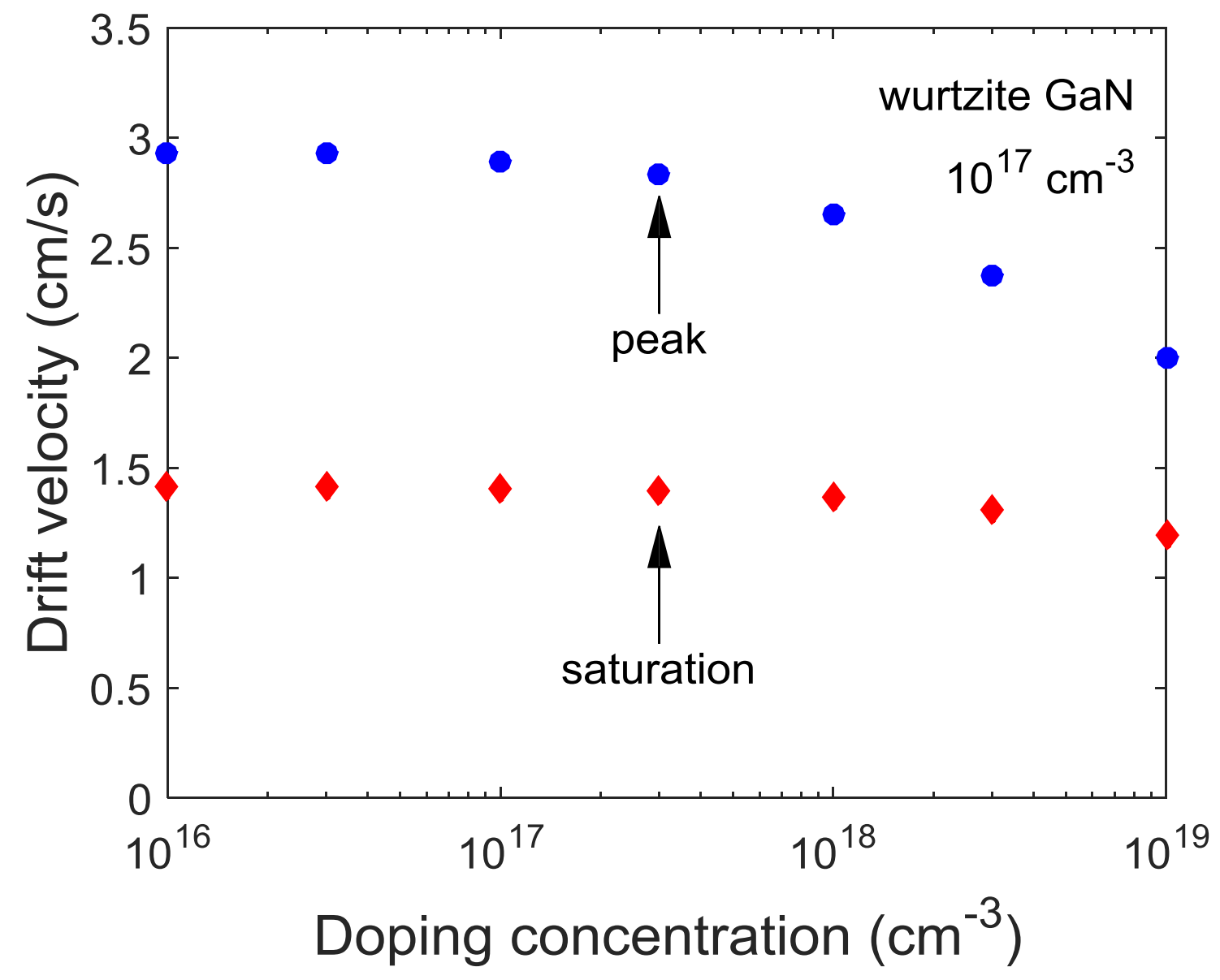

Figure 3.22.b: The peak and saturation electron drift velocities associated with bulk wurtzite GaN as a function of the doping concentration. These results are determined from the results of Monte Carlo simulations of electron transport within bulk wurtzite GaN. For all cases, I have assumed a crystal temperature of $300 \mathrm{~K}$. This figure has been modified from @ Siddiqua, P. \& O'Leary, S.K. (2018). Electron transport within the wurtzite and zinc-blende phases of gallium nitride and indium nitride, Journal of Materials Science, 29, 3511-3567. Page 3539. Adapted with permission from publisher. The online version of this figure is depicted in color. 


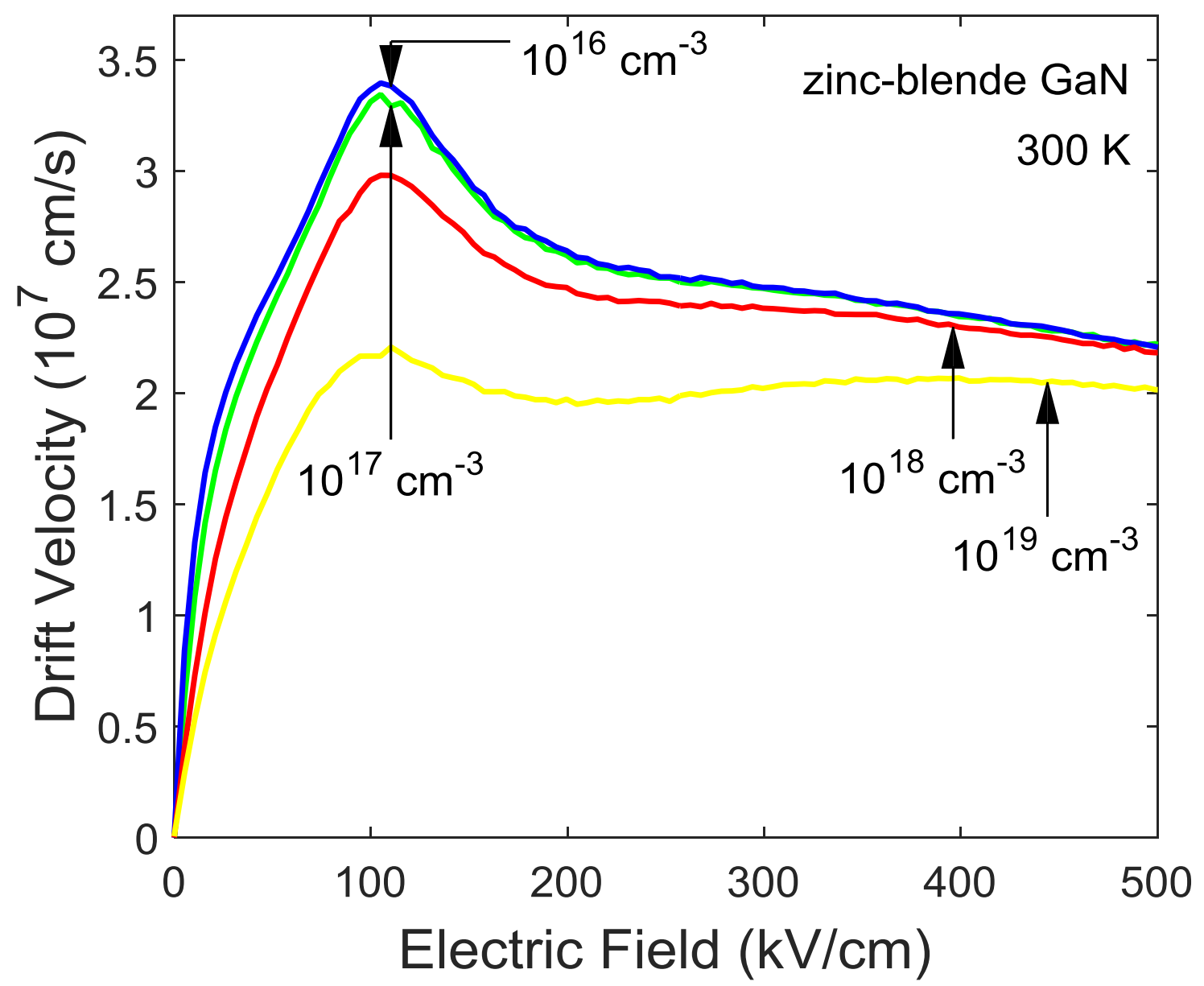

Figure 3.23.a: The velocity-field characteristics associated with bulk zinc-blende GaN for various doping concentrations. For all cases, I have assumed a crystal temperature of $300 \mathrm{~K}$. This figure has been modified from @ (c) Siddiqua, P. \& O'Leary, S.K. (2018). Electron transport within the wurtzite and zinc-blende phases of gallium nitride and indium nitride, Journal of Materials Science, 29, 3511-3567. Page 3540. Adapted with permission from publisher. The online version of this figure is depicted in color. 


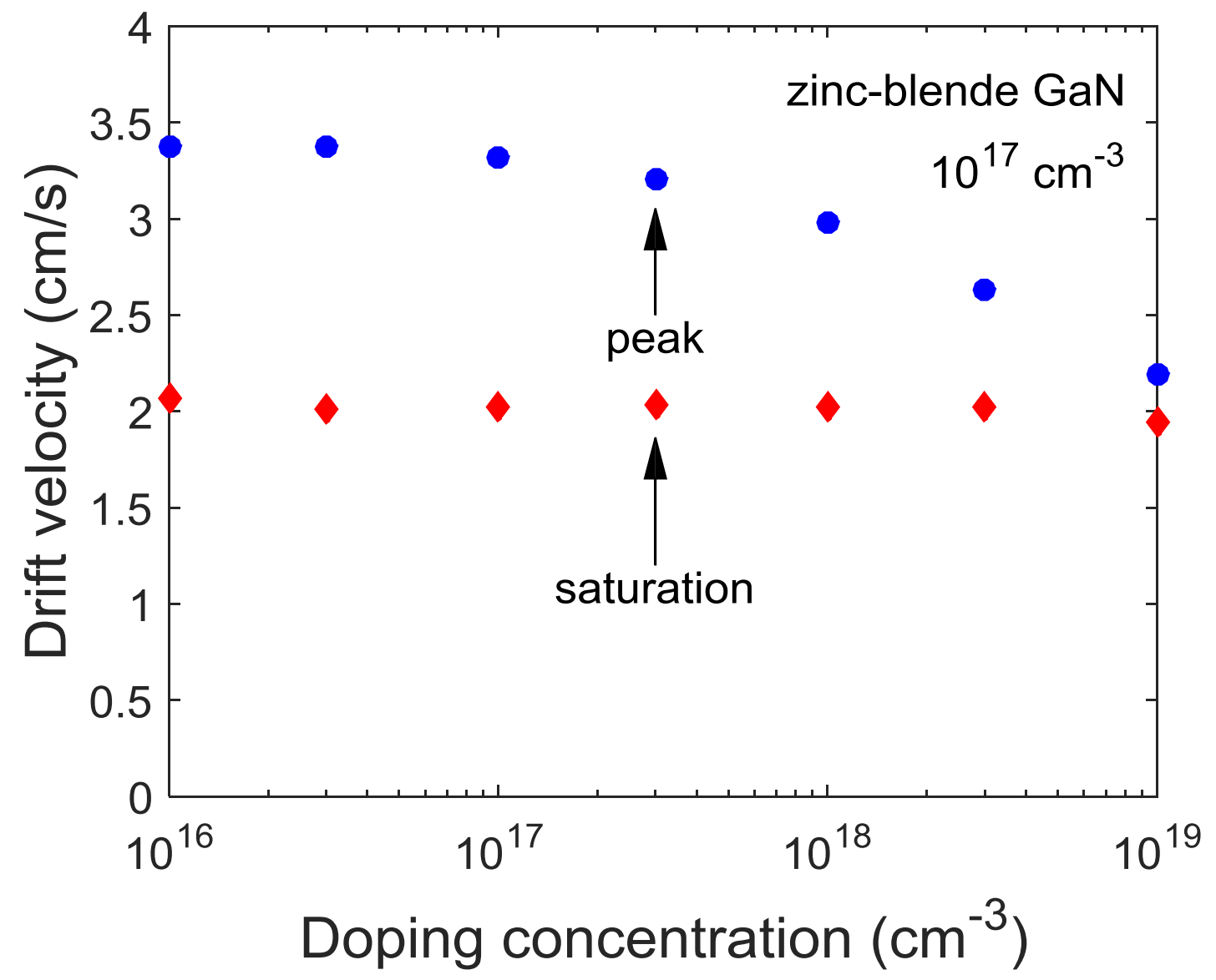

Figure 3.23.b: The peak and saturation electron drift velocities associated with bulk zinc-blende GaN as a function of the doping concentration. These results are determined from the results of Monte Carlo simulations of electron transport within bulk wurtzite GaN. For all cases, I have assumed a crystal temperature of 300K. This figure has been modified from (C) Siddiqua, P. \& O'Leary, S.K. (2018). Electron transport within the wurtzite and zinc-blende phases of gallium nitride and indium nitride, Journal of Materials Science, 29, 3511-3567. Page 3540. Adapted with permission from publisher. The online version of this figure is depicted in color. 


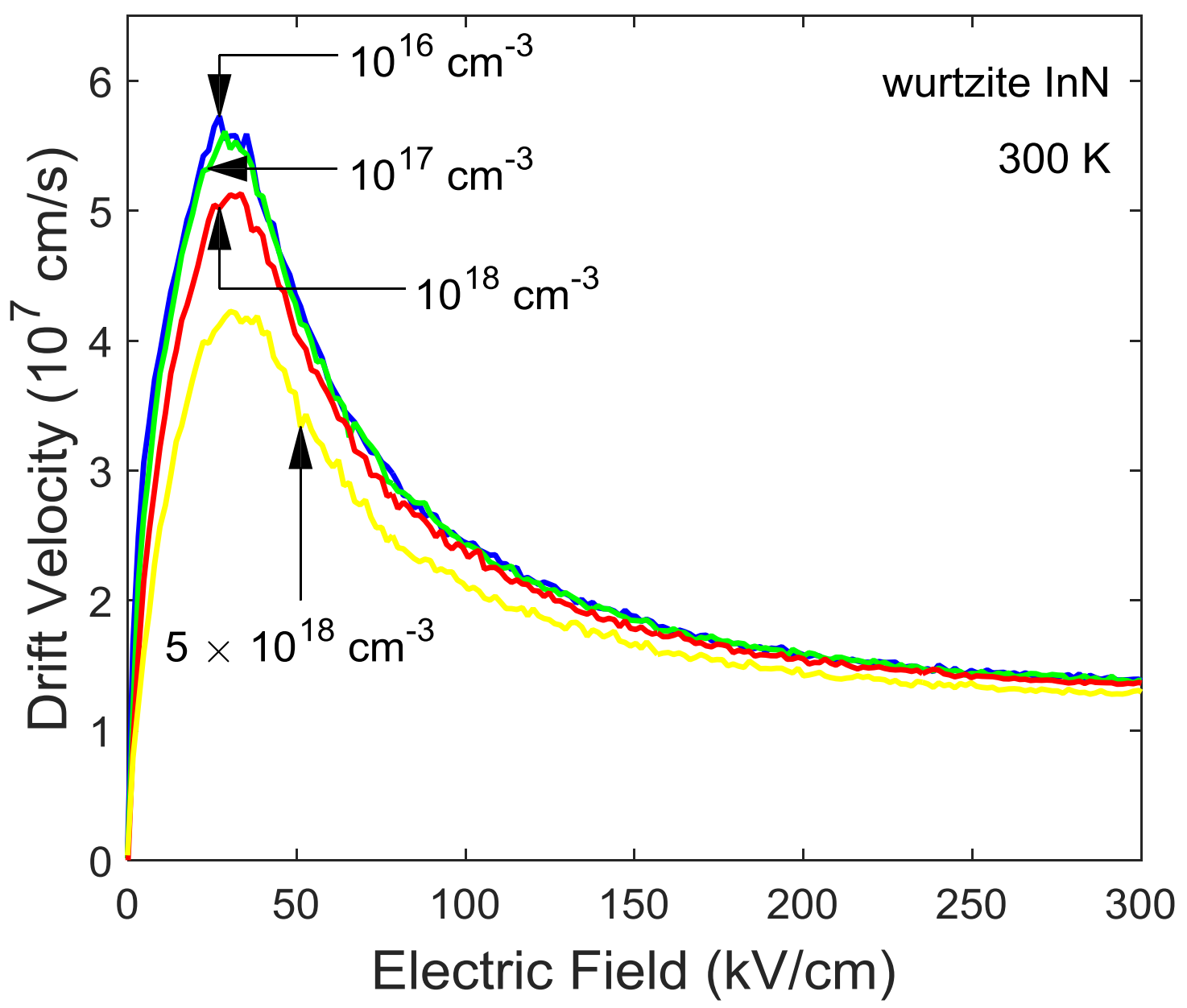

Figure 3.24.a: The velocity-field characteristics associated with bulk wurtzite $\ln N$ for various doping concentrations. For all cases, I have assumed a crystal temperature of $300 \mathrm{~K}$. This figure has been modified from @ Siddiqua, P. \& O'Leary, S.K. (2018). Electron transport within the wurtzite and zinc-blende phases of gallium nitride and indium nitride, Journal of Materials Science, 29, 3511-3567. Page 3540. Adapted with permission from publisher. The online version of this figure is depicted in color. 


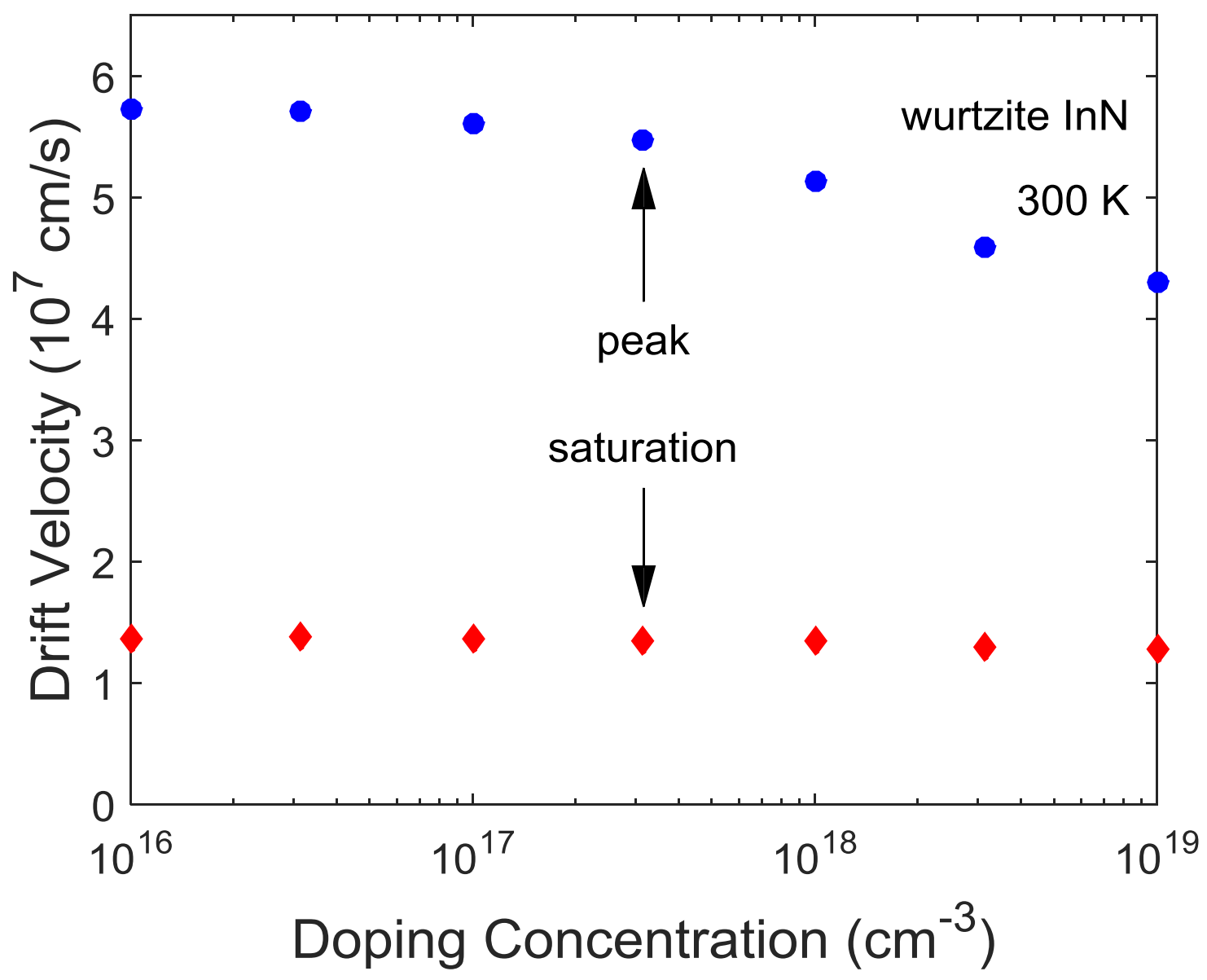

Figure 3.24.b: The peak and saturation electron drift velocities associated with bulk wurtzite InN as a function of the doping concentration. These results are determined from the results of Monte Carlo simulations of electron transport within bulk wurtzite InN. For all cases, I have assumed a crystal temperature of $300 \mathrm{~K}$. This figure has been modified from ( ) Siddiqua, P. \& O'Leary, S.K. (2018). Electron transport within the wurtzite and zinc-blende phases of gallium nitride and indium nitride, Journal of Materials Science, 29, 3511-3567. Page 3540. Adapted with permission from publisher. The online version of this figure is depicted in color. 


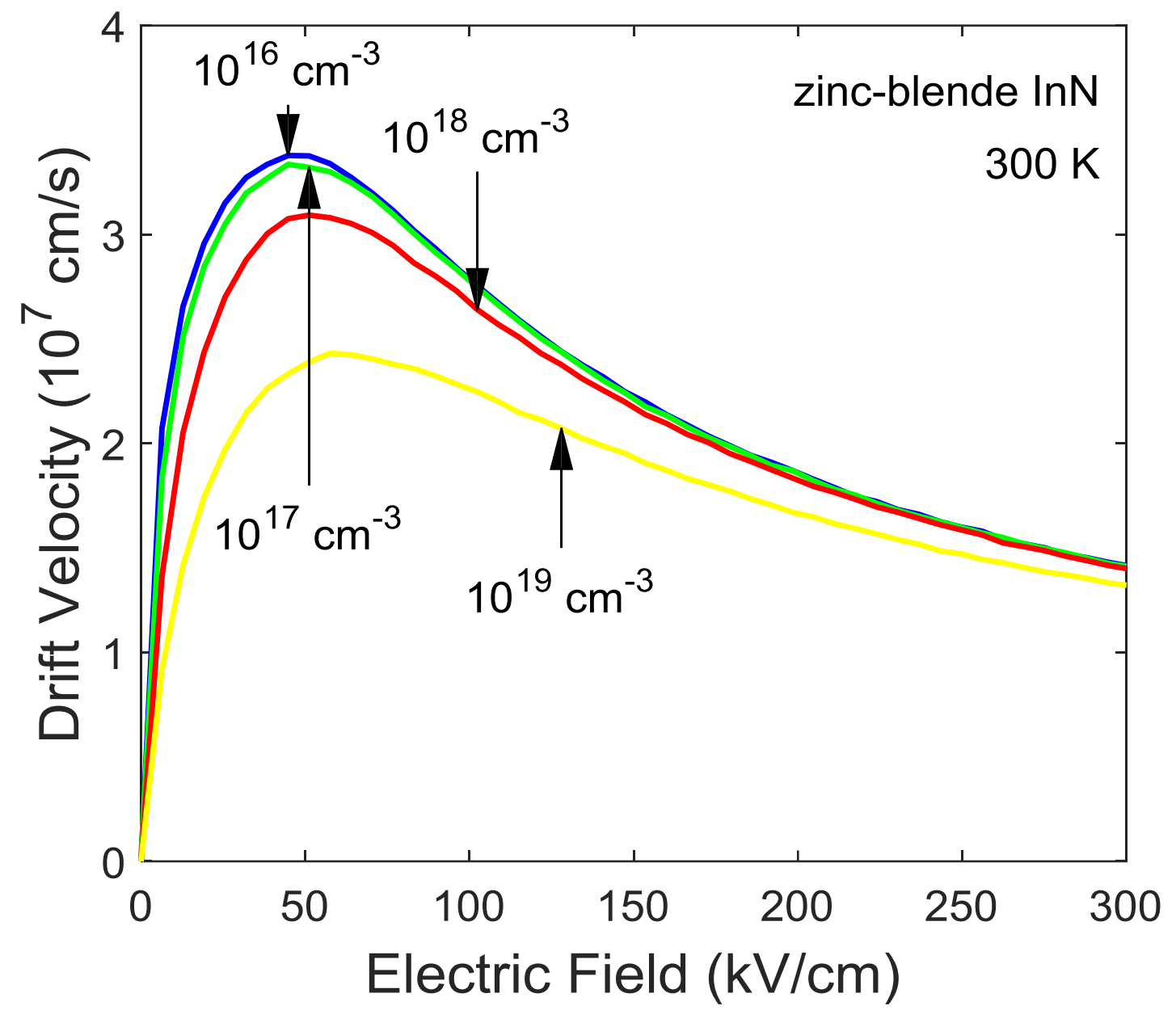

Figure 3.25.a: The velocity-field characteristics associated with bulk zinc-blende $\ln N$ for various doping concentrations. For all cases, I have assumed a crystal temperature of $300 \mathrm{~K}$. This figure has been modified from (c) Siddiqua, P. \& O'Leary, S.K. (2018). Electron transport within the wurtzite and zinc-blende phases of gallium nitride and indium nitride, Journal of Materials Science, 29, 3511-3567. Page 3541. Adapted with permission from publisher. The online version of this figure is depicted in color. 


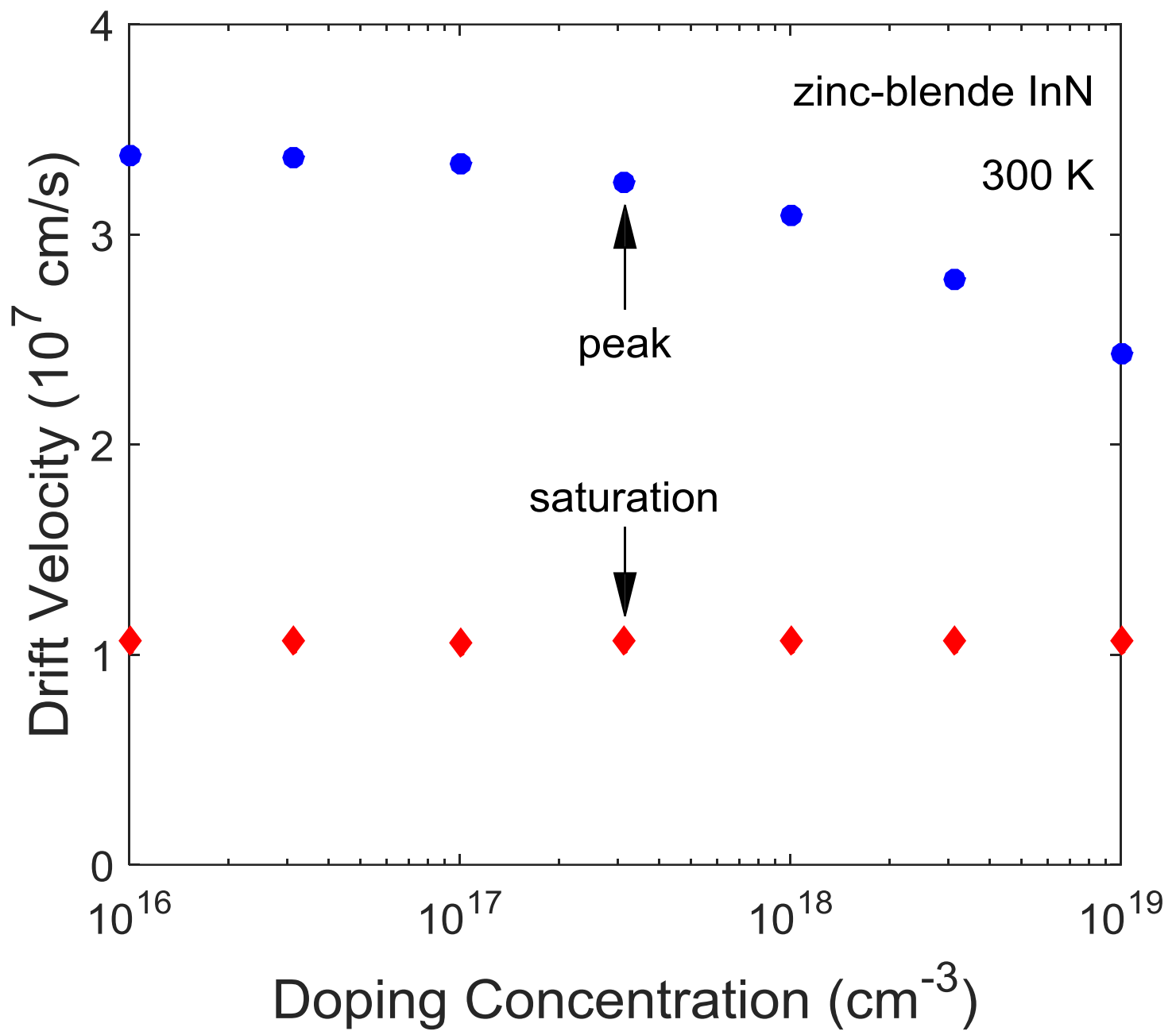

Figure 3.25.b: The peak and saturation electron drift velocities associated with bulk zinc-blende $\ln N$ as a function of the doping concentration. These results are determined from the results of Monte Carlo simulations of electron transport within bulk zinc-blende InN. For all cases, I have assumed a crystal temperature of $300 \mathrm{~K}$. This figure has been modified from @ S Siddiqua, P. \& O'Leary, S.K. (2018). Electron transport within the wurtzite and zinc-blende phases of gallium nitride and indium nitride, Journal of Materials Science, 29, 3511-3567. Page 3541. Adapted with permission from publisher. The online version of this figure is depicted in color. 


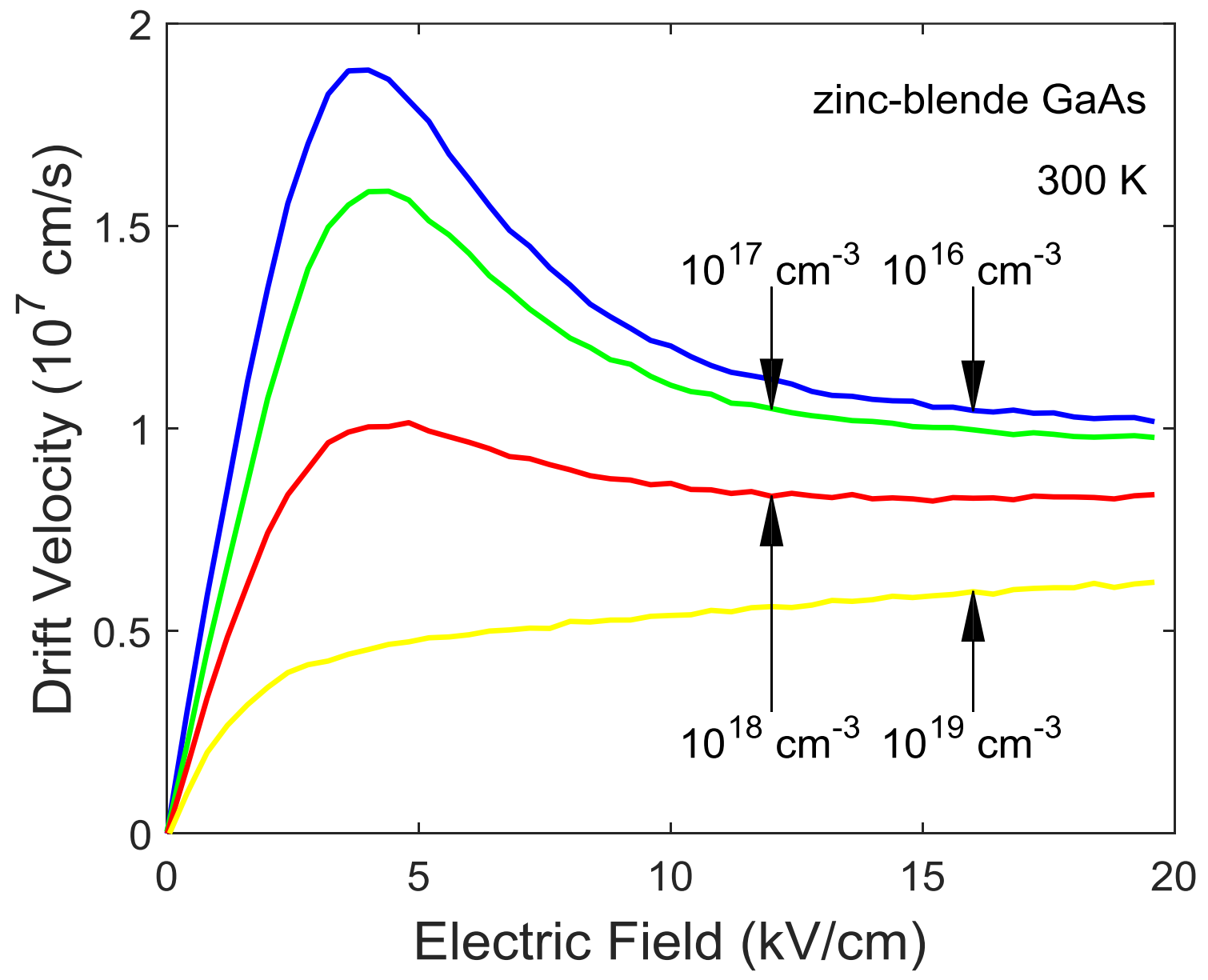

Figure 3.26.a: The velocity-field characteristics associated with bulk zinc-blende GaAs for various doping concentrations. For all cases, I have assumed a crystal temperature of $300 \mathrm{~K}$. This figure has been modified from (c) Siddiqua, P. \& O'Leary, S.K. (2018). Electron transport within the wurtzite and zinc-blende phases of gallium nitride and indium nitride, Journal of Materials Science, 29, 3511-3567. Page 3541. Adapted with permission from publisher. The online version of this figure is depicted in color. 


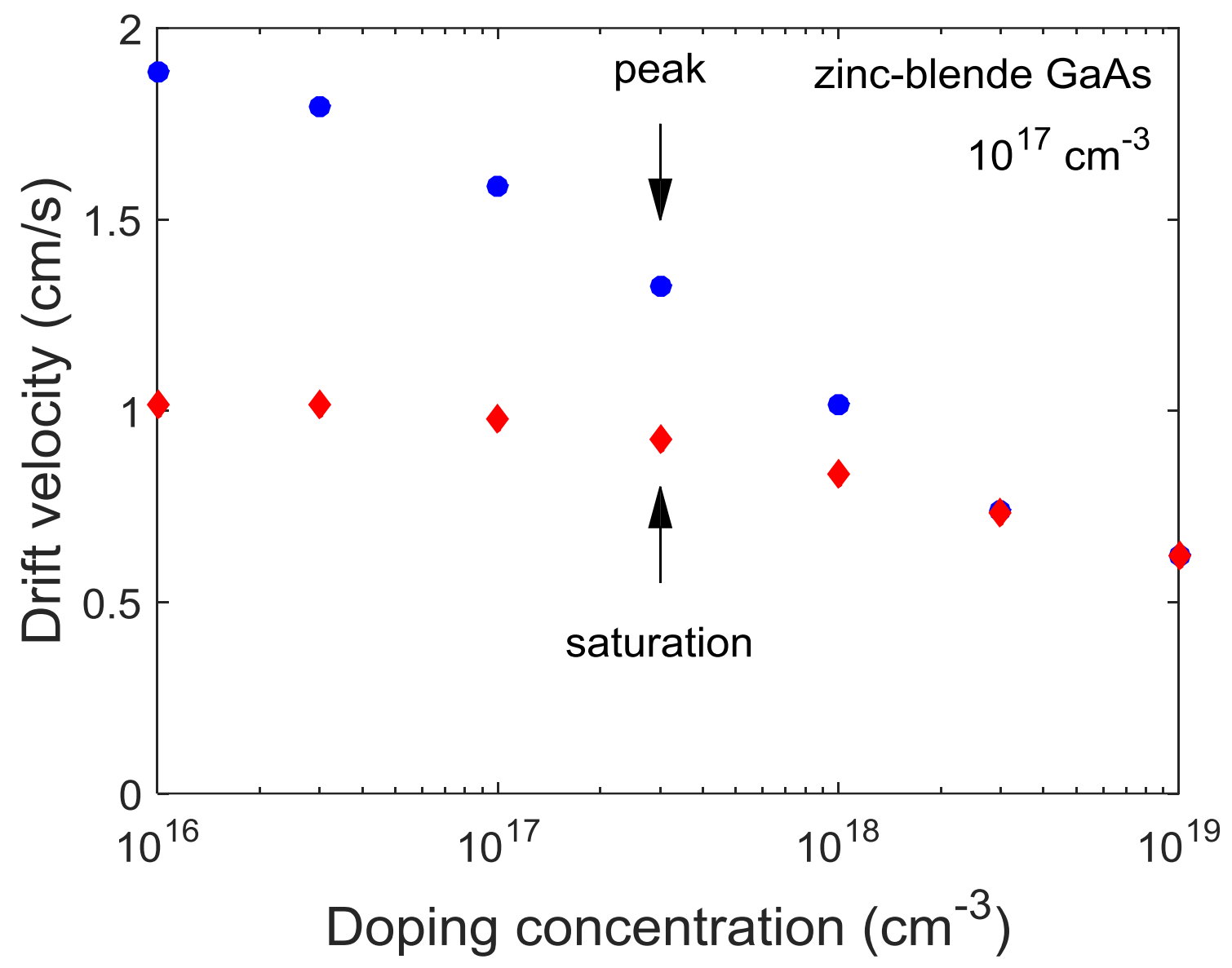

Figure 3.26.b: The peak and saturation electron drift velocities associated with bulk zinc-blende GaAs as a function of the doping concentration. These results are determined from the results of Monte Carlo simulations of electron transport within bulk zinc-blende GaAs. For all cases, I have assumed a crystal temperature of $300 \mathrm{~K}$. This figure has been modified from @ Siddiqua, P. \& O'Leary, S.K. (2018). Electron transport within the wurtzite and zinc-blende phases of gallium nitride and indium nitride, Journal of Materials Science, 29, 3511-3567. Page 3541. Adapted with permission from publisher. The online version of this figure is depicted in color. 
esponding steady-state electron drift velocity by a considerable margin for appropriate selections of the applied electric field strength. Shur and Eastman [160] explored the device implications of transient electron transport, and demonstrated that substantial improvements in the device performance may be achieved as a consequence. Heiblum et al. [161] made the first direct experimental observation of transient electron transport within GaAs. Since then, there have been a number of experimental investigations into the transient electron transport within III-V compound semiconductors; see, for example [162-164].

Thus far, very little research has been invested into the study of the transient electron transport within the semiconductors under consideration in this analysis, i.e., the wurtzite and zinc-blende phases of $\mathrm{GaN}$ and InN. In 1997, Foutz et al. [64] examined transient electron transport within the wurtzite and zinc-blende phases of GaN. In particular, they examined how electrons, initially in thermal equilibrium, respond to the sudden application of a constant electric field. In devices with dimensions greater than $0.2 \mu \mathrm{m}$, they found that steady-state electron transport is expected to dominate device performance. For devices with smaller dimensions, however, with the application of a sufficiently high electric field strength, they found that the transient electron drift velocity can considerably overshoot the corresponding steady-state electron drift velocity. This velocity overshoot was found to be comparable with that which occurs within GaAs. A subsequent analysis, reported by Foutz et al. [132] in 1999, contrasted the nature of the transient electron transport over a broad range of III-V nitride semiconductors.

Following in the tradition of Foutz et al. [64,132], we examine how an ensemble of electrons, initially in thermal equilibrium, respond to the application of a constant electric field for the cases of wurtzite GaN, zinc-blende GaN, wurtzite InN, and zinc-blende InN. In particular, Figures $3.27,3.28,3.29$, and 3.30 depict the transient electron drift velocity as a function of the distance displaced since the electric field was initially applied, for a number of electric field strength selections, for the cases of wurtzite GaN, zinc-blende GaN, wurtzite InN, and zinc-blende 
$\ln N$, respectively. For all cases, the crystal temperature is set to $300 \mathrm{~K}$ and the doping concentration is set to $10^{17} \mathrm{~cm}^{-3}$. These results are obtained from Monte Carlo electron transport simulations. For each transient electron transport simulation, the motion of ten-thousand electrons is considered.

Focusing initially on the case of wurtzite GaN (see Figure 3.27), we note that for the applied electric field strength selections 70 and $140 \mathrm{kV} / \mathrm{cm}$, the electron drift velocity reaches steady-state very quickly, with little or no velocity overshoot. In contrast, for applied electric field strength selections above $140 \mathrm{kV} / \mathrm{cm}$, significant velocity overshoot occurs. This result suggests that in wurtzite $\mathrm{GaN}, 140 \mathrm{kV} / \mathrm{cm}$ is a critical field for the onset of velocity overshoot effects. As was mentioned in Sections 3.2 and $3.7,140 \mathrm{kV} / \mathrm{cm}$ also corresponds to the peak in the velocityfield characteristic associated with wurtzite GaN; recall Figures 3.1 and 3.16. Steady-state Monte Carlo simulations suggest that this is the point at which significant upper conduction band valley occupation begins to occur; recall Figure 3.3. This tells us that the velocity overshoot that occurs within this material is related to the transfer of electrons to the upper energy conduction band valleys. Similar results are found for the cases of zinc-blende GaN and wurtzite InN. For the case of zinc-blende $\operatorname{InN}$, however, the clearly observed velocity overshoot is related to the sudden increase in the electron effective mass that accompanies higher electron energies, i.e., increases in the electron effective mass within the lowest energy conduction band itself quickly act to dampen the corresponding transient electron drift velocity [157]. For bench-marking purposes, the case of zinc-blende GaAs is also considered; see Figure 3.31. A similar result is found for the case of this material. The critical field strengths for the onset of velocity overshoot are found to be $110 \mathrm{kV} / \mathrm{cm}$ for the case of zinc-blende $\mathrm{GaN}, 30 \mathrm{kV} / \mathrm{cm}$ for the case of wurtzite $\mathrm{InN}, 50 \mathrm{kV} / \mathrm{cm}$ for the case of zinc-blende $\mathrm{InN}$, and $4 \mathrm{kV} / \mathrm{cm}$ for the case of zinc-blende GaAs; recall Figures 3.28, $3.29,3.30$, and 3.31 , respectively. We note that, for all cases, these critical electric field strengths are identical to the respective peak field strengths; recall Figures 3.4, 3.7, 3.10, 3.13, and 3.16. 


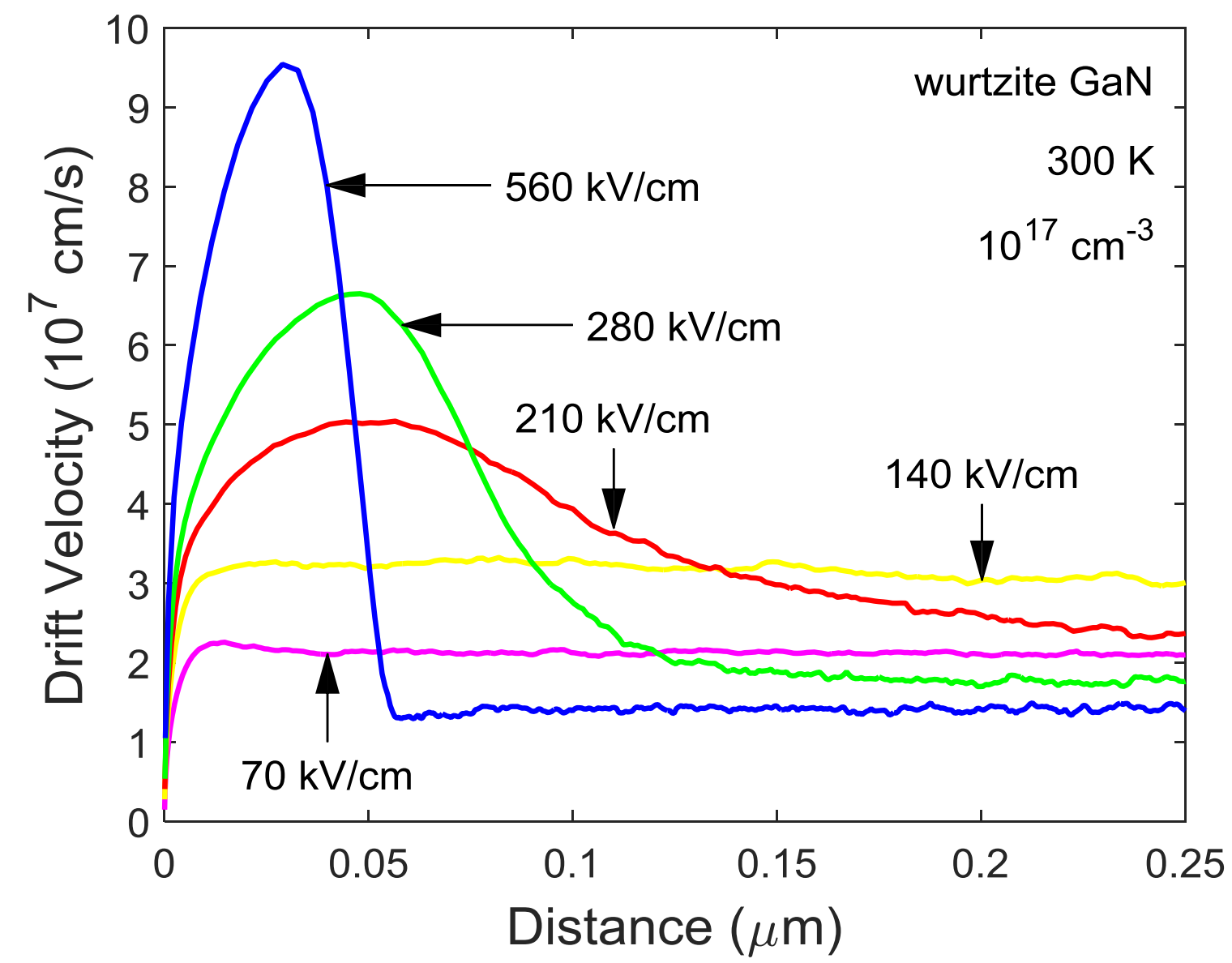

Figure 3.27: The electron drift velocity as a function of the distance displaced since the application of the electric field, for various applied electric field strength selections, for the case of bulk wurtzite GaN. For all cases, I have assumed an initial zero-field electron distribution, a crystal temperature of $300 \mathrm{~K}$, and a doping concentration of $10^{17} \mathrm{~cm}^{-3}$. This figure has been modified from (c) Siddiqua, P. \& O'Leary, S.K. (2018). Electron transport within the wurtzite and zinc-blende phases of gallium nitride and indium nitride, Journal of Materials Science, 29, 3511-3567. Page 3542. Adapted with permission from publisher. The online version of this figure is depicted in color. 


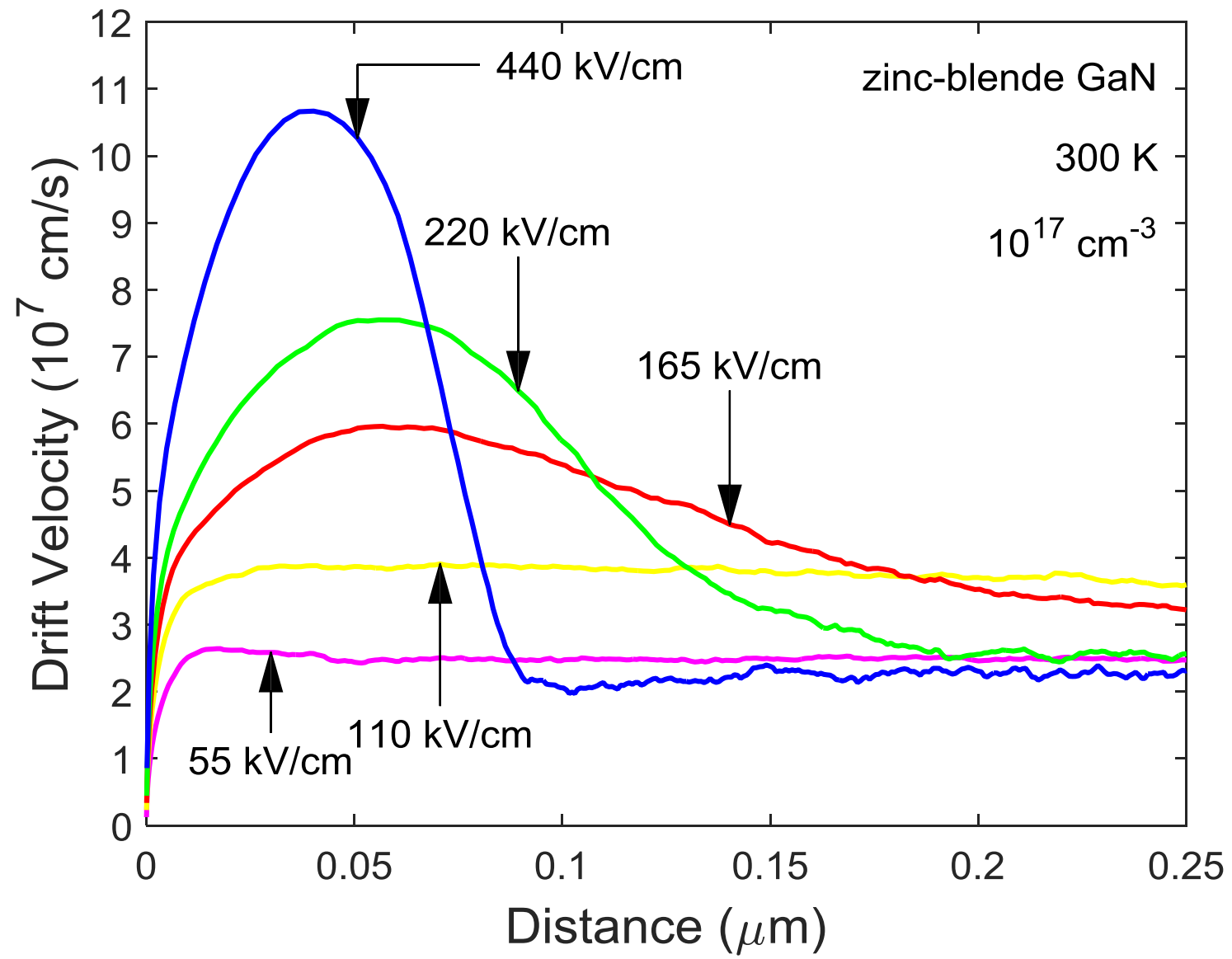

Figure 3.28: The electron drift velocity as a function of the distance displaced since the application of the electric field, for various applied electric field strength selections, for the case of bulk zinc-blende GaN. For all cases, I have assumed an initial zero-field electron distribution, a crystal temperature of $300 \mathrm{~K}$, and a doping concentration of $10^{17} \mathrm{~cm}^{-3}$. This figure has been modified from (C Siddiqua, P. \& O'Leary, S.K. (2018). Electron transport within the wurtzite and zinc-blende phases of gallium nitride and indium nitride, Journal of Materials Science, 29, 3511-3567. Page 3543. Adapted with permission from publisher. The online version of this figure is depicted in color. 


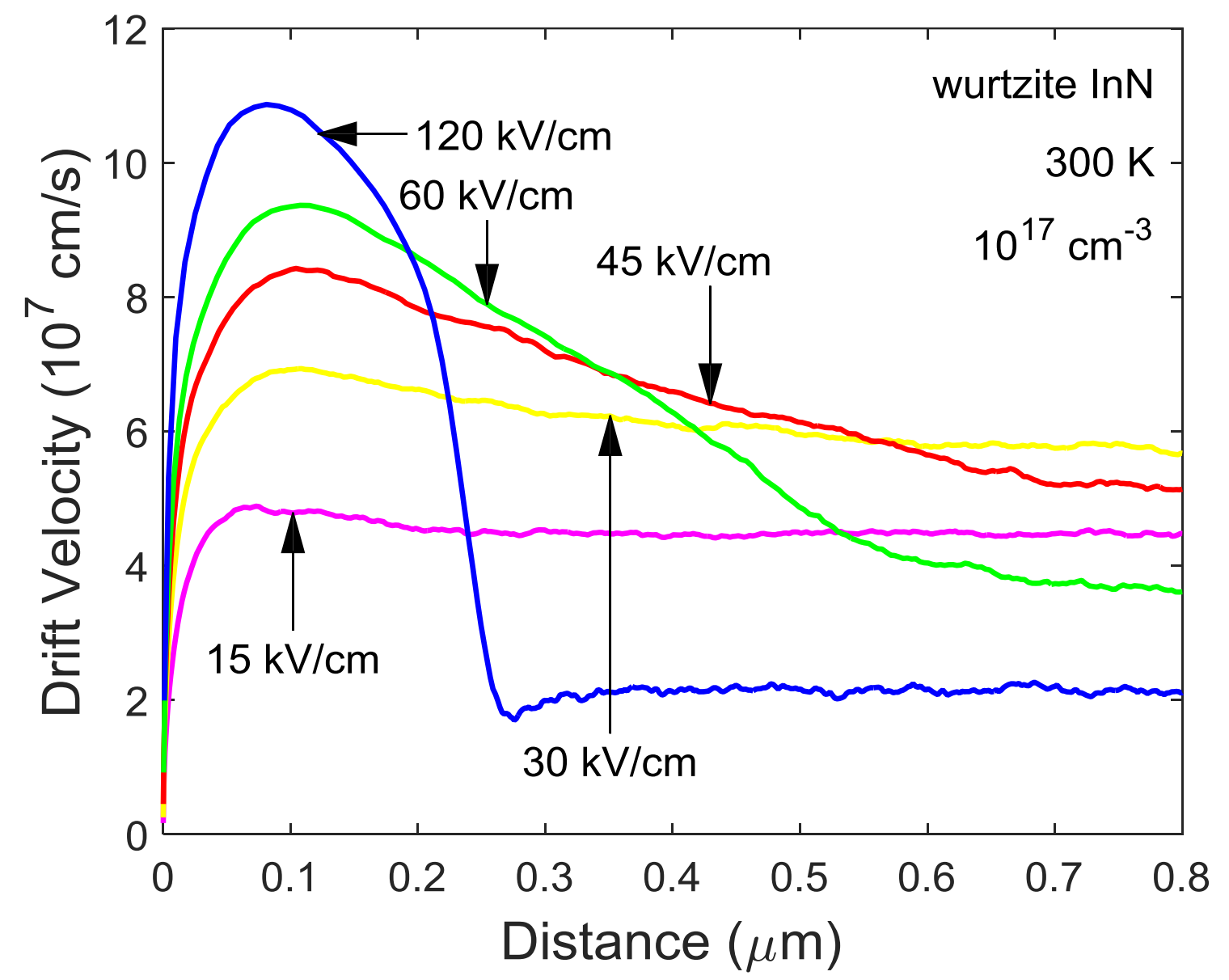

Figure 3.29: The electron drift velocity as a function of the distance displaced since the application of the electric field, for various applied electric field strength selections, for the case of bulk wurtzite InN. For all cases, I have assumed an initial zero-field electron distribution, a crystal temperature of $300 \mathrm{~K}$, and a doping concentration of $10^{17} \mathrm{~cm}^{-3}$. This figure has been modified from @ Siddiqua, P. \& O'Leary, S.K. (2018). Electron transport within the wurtzite and zinc-blende phases of gallium nitride and indium nitride, Journal of Materials Science, 29, 3511-3567. Page 3543. Adapted with permission from publisher. The online version of this figure is depicted in color. 


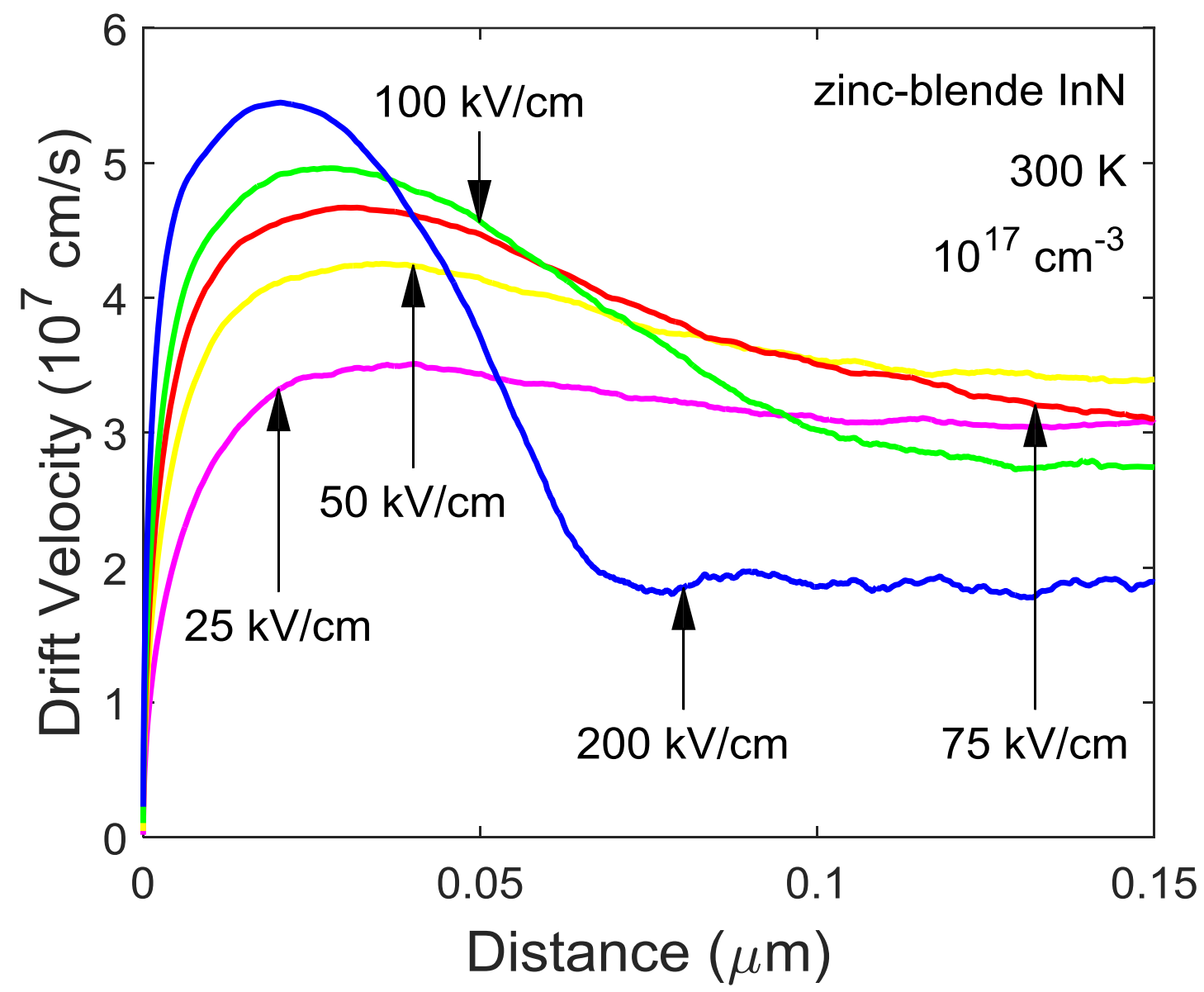

Figure 3.30: The electron drift velocity as a function of the distance displaced since the application of the electric field, for various applied electric field strength selections, for the case of bulk zinc-blende InN. For all cases, I have assumed an initial zero-field electron distribution, a crystal temperature of $300 \mathrm{~K}$, and a doping concentration of $10^{17} \mathrm{~cm}^{-3}$. This figure has been modified from (C) Siddiqua, P. \& O'Leary, S.K. (2018). Electron transport within the wurtzite and zinc-blende phases of gallium nitride and indium nitride, Journal of Materials Science, 29, 3511-3567. Page 3543. Adapted with permission from publisher. The online version of this figure is depicted in color. 


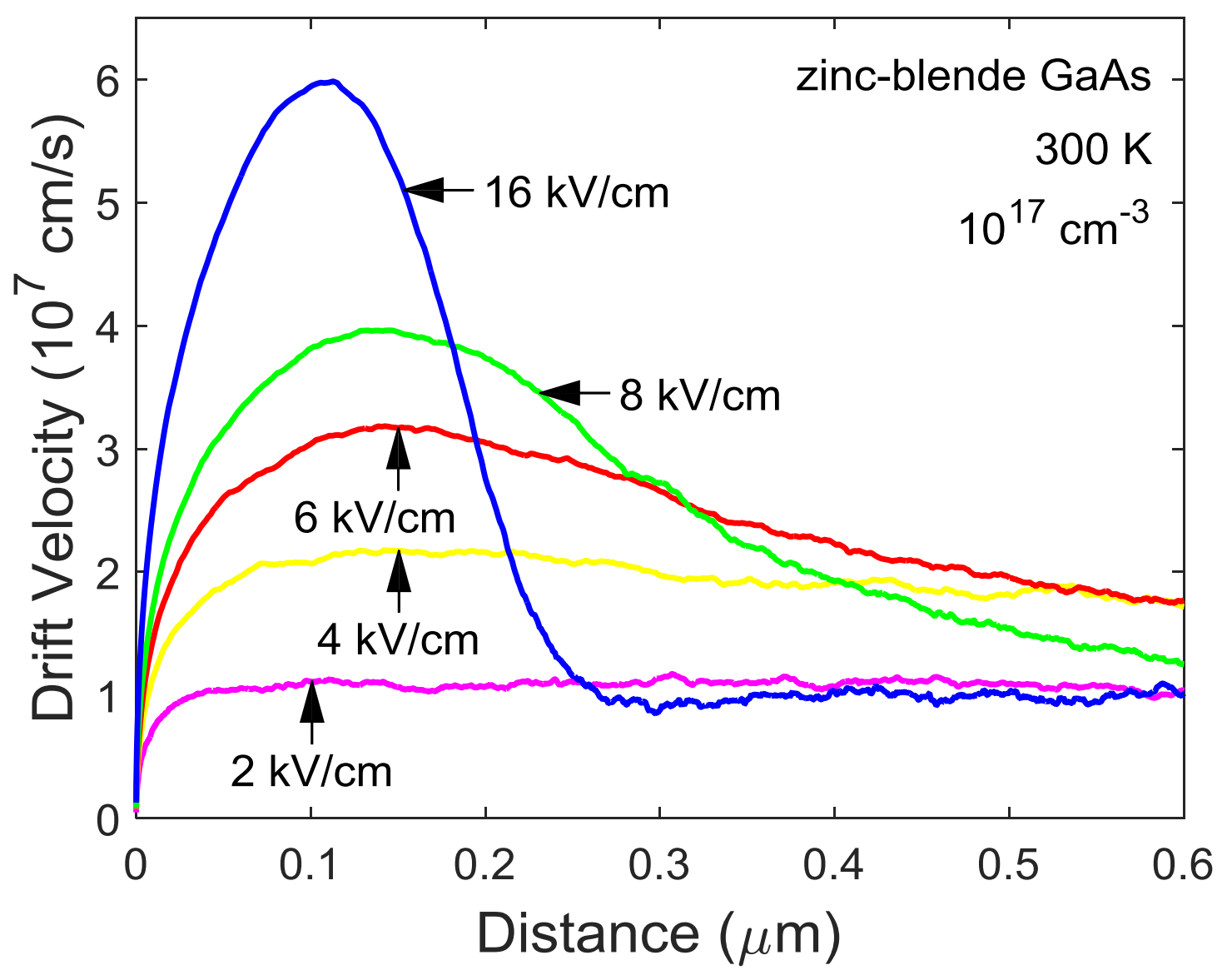

Figure 3.31: The electron drift velocity as a function of the distance displaced since the application of the electric field, for various applied electric field strength selections, for the case of bulk zinc-blende GaAs. For all cases, I have assumed an initial zero-field electron distribution, a crystal temperature of $300 \mathrm{~K}$, and a doping concentration of $10^{17} \mathrm{~cm}^{-3}$. This figure has been modified from @ Siddiqua, P. \& O'Leary, S.K. (2018). Electron transport within the wurtzite and zinc-blende phases of gallium nitride and indium nitride, Journal of Materials Science, 29, 3511-3567. Page 3544. Adapted with permission from publisher. The online version of this figure is depicted in color. 
I now compare the transient electron transport characteristics for the various materials. From Figures 3.27, 3.28, 3.29, 3.30, and 3.31, it is clear that certain materials exhibit higher peak overshoot velocities and longer overshoot relaxation times. It is not possible to fairly compare these different semiconductors by applying the same applied electric field strength for all of the materials, as these transient effects occur over such a disparate range of applied electric field strengths for each material. In order to facilitate such a comparison, we choose a field strength equal to twice the critical applied electric field strength for each material, i.e., $280 \mathrm{kV} / \mathrm{cm}$ for wurtzite GaN, 220 kV/cm for zinc-blende GaN, 60 kV/cm for wurtzite InN, 100 kV/cm for zincblende $\operatorname{lnN}$, and $8 \mathrm{kV} / \mathrm{cm}$ for zinc-blende GaAs. Figure 3.32.a shows such a comparison of the velocity overshoot effects amongst the four materials considered in this analysis, i.e., the wurtzite and zinc-blende phases of $\mathrm{GaN}$ and $\mathrm{InN}$, and zinc-blende GaAs. The corresponding peak transient electron drift velocities are depicted in Figure 3.32.b. It is clear that among the semiconductors considered, wurtzite $\operatorname{InN}$ exhibits superior transient electron transport characteristics. In particular, wurtzite InN has the largest overshoot velocity and the distance over which this overshoot occurs, in excess of $0.6 \mu \mathrm{m}$, is longer than that associated with most of the other materials considered in this analysis; a detailed examination, beyond the scale of Figure 3.32.a, demonstrates that the velocity overshoot associated with wurtzite $\ln N$ for the electric field strength set to $60 \mathrm{kV} / \mathrm{cm}$, the crystal temperature set to $300 \mathrm{~K}$, and the doping concentration set to $10^{17} \mathrm{~cm}^{-3}$, lasts for about $0.65 \mu \mathrm{m}$. Zinc-blende GaAs, for the electric field strength set to 8 $\mathrm{kV} / \mathrm{cm}$, the crystal temperature set to $300 \mathrm{~K}$, and the doping concentration set to $10^{17} \mathrm{~cm}^{-3}$, is found to exhibit a slightly longer overshoot relaxation distance, approximately $0.7 \mu \mathrm{m}$, but the electron drift velocity exhibited by zinc-blende GaAs is less than that of wurtzite GaN, wurtzite InN, and zinc-blende InN over most displacements. 


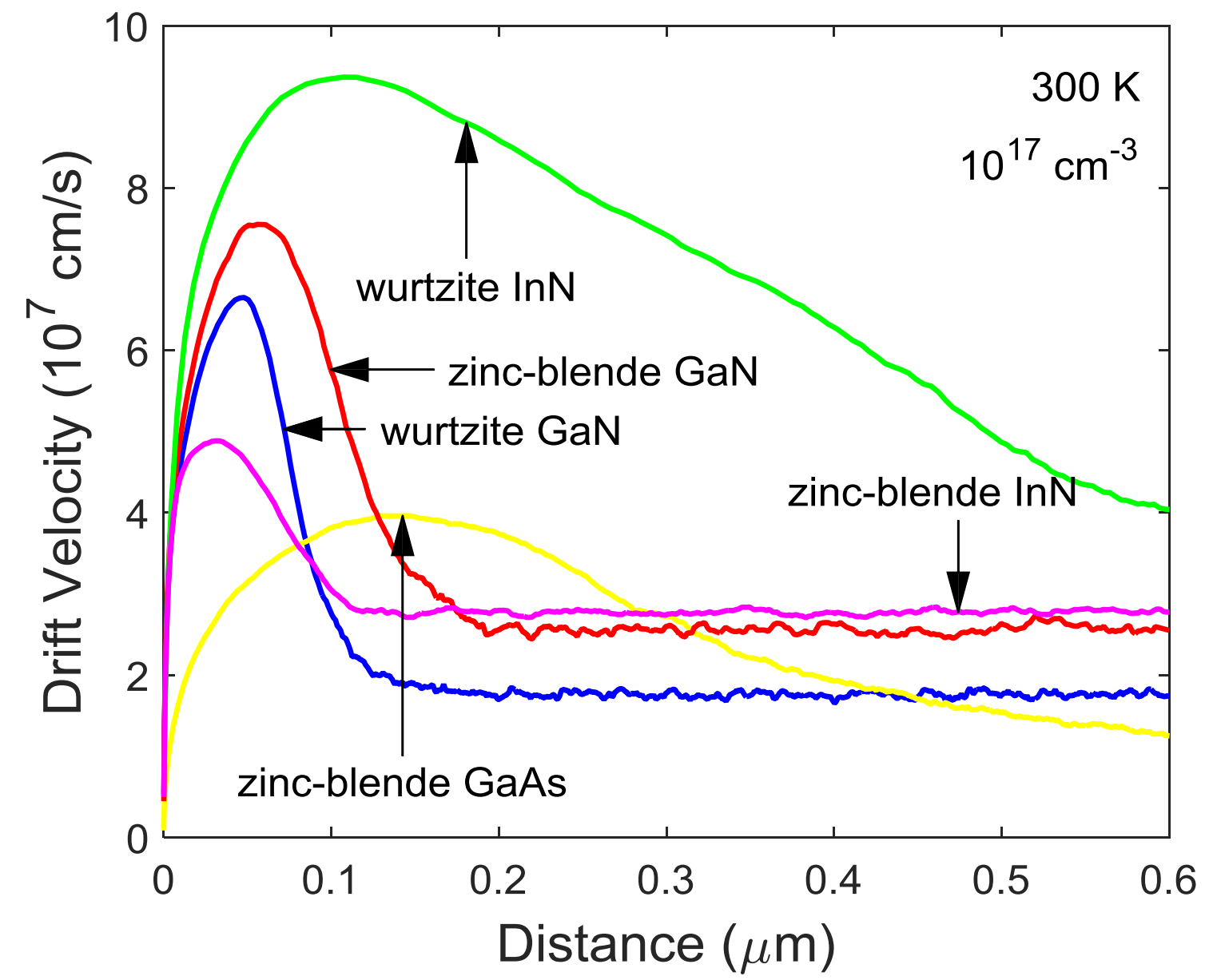

Figure 3.32.a: A comparison of the velocity overshoot amongst the bulk semiconductors considered in this analysis, i.e., the wurtzite and zinc-blende phases of $\mathrm{GaN}$ and $\mathrm{InN}$, with that associated with bulk zincblende GaAs. For all cases, the crystal temperature is set to $300 \mathrm{~K}$ and the doping concentration is set to $10^{17} \mathrm{~cm}^{-3}$. The applied electric field strengths chosen correspond to twice the peak field strengths for all cases, i.e., $280 \mathrm{kV} / \mathrm{cm}$ for wurtzite GaN, $220 \mathrm{kV} / \mathrm{cm}$ for zinc-blende GaN, $60 \mathrm{kV} / \mathrm{cm}$ for wurtzite InN, 100 $\mathrm{kV} / \mathrm{cm}$ for zinc-blende $\mathrm{InN}$, and $8 \mathrm{kV} / \mathrm{cm}$ for zinc-blende GaAs. This figure has been modified from (C) Siddiqua, P. \& O'Leary, S.K. (2018). Electron transport within the wurtzite and zinc-blende phases of gallium nitride and indium nitride, Journal of Materials Science, 29, 3511-3567. Page 3546. Adapted with permission from publisher. The online version of this figure is depicted in color. 


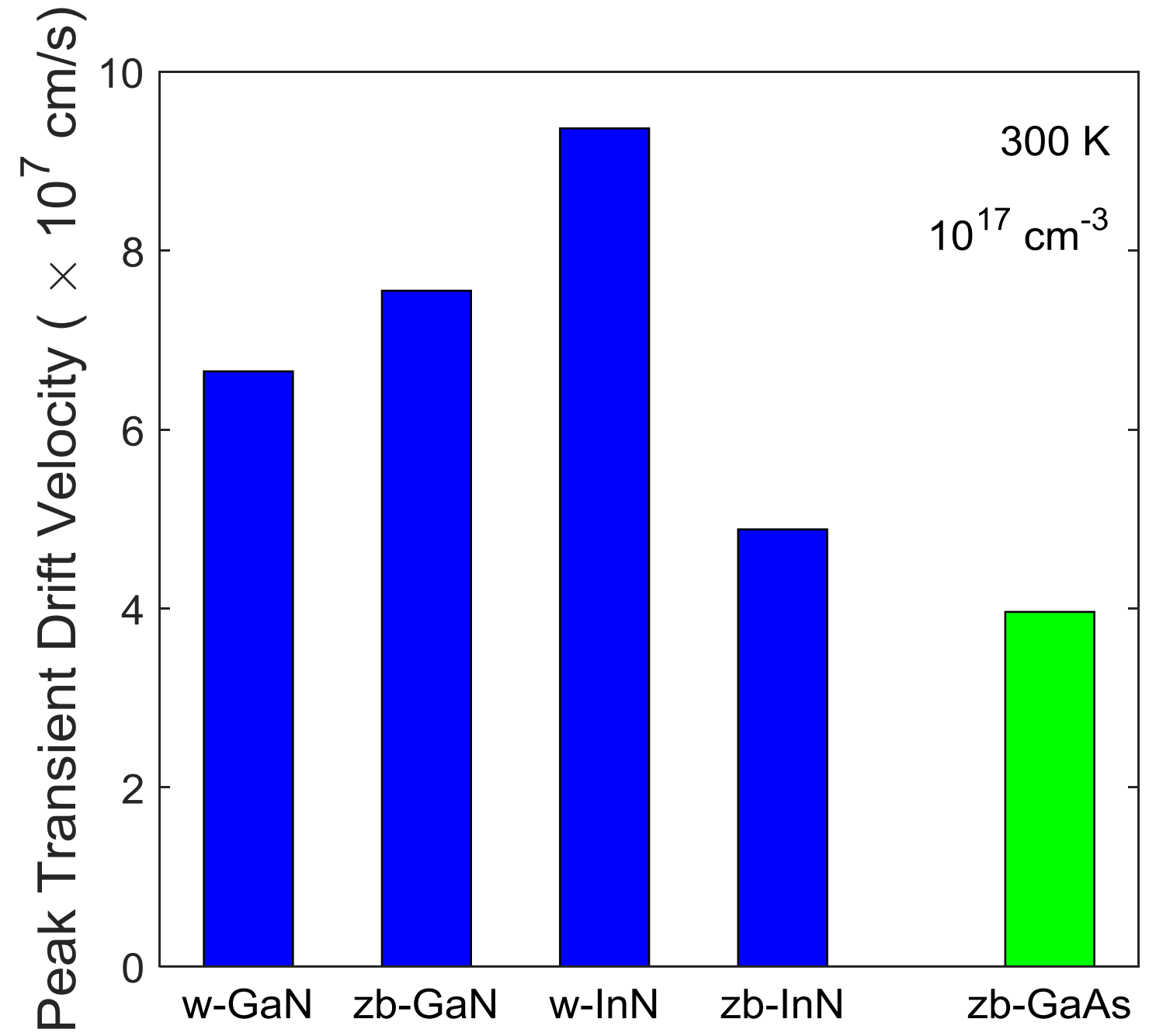

Figure 3.32.b: A comparison of the peak transient electron drift velocities associated with the bulk semiconductors considered in this analysis, i.e., the wurtzite and zinc-blende phases of $\mathrm{GaN}$ and InN, with that associated with bulk zinc-blende GaAs. The crystal temperature is set to $300 \mathrm{~K}$ and the doping concentration is set to $10^{17} \mathrm{~cm}^{-3}$ for all cases. The applied electric field strength is set to twice the peak field strength for all cases, i.e., $280 \mathrm{kV} / \mathrm{cm}$ for wurtzite $\mathrm{GaN}, 220 \mathrm{kV} / \mathrm{cm}$ for zinc-blende $\mathrm{GaN}, 60 \mathrm{kV} / \mathrm{cm}$ for wurtzite $\operatorname{lnN}, 100 \mathrm{kV} / \mathrm{cm}$ for zinc-blende $\mathrm{InN}$, and $8 \mathrm{kV} / \mathrm{cm}$ for zinc-blende GaAs. This figure has been modified from @ Siddiqua, P. \& O'Leary, S.K. (2018). Electron transport within the wurtzite and zinc-blende phases of gallium nitride and indium nitride, Journal of Materials Science, 29, 3511-3567. Page 3546. Adapted with permission from publisher. The online version of this figure is depicted in color. 


\subsection{Electron transport conclusions}

In this chapter, steady-state and transient electron transport results, corresponding to the wurtzite and zinc-blende phases of $\mathrm{GaN}$ and $\mathrm{InN}$, were presented, these results being obtained from our Monte Carlo simulations of electron transport within these materials. Steady-state electron transport was the dominant theme of our analysis. In order to aid in the understanding of these electron transport characteristics, a comparison was made between these results and those associated with zinc-blende GaAs. Finally, we presented some key transient electron transport results, these results indicating that the transient electron transport that occurs within wurtzite $\ln N$ is the most pronounced of all of the materials under consideration in this review, i.e., the wurtzite and zinc-blende phases of $\mathrm{GaN}$ and $\mathrm{InN}$, and zinc-blende GaAs. 


\section{Chapter 4: Electron transport within wide energy gap semiconductors: A review}

\subsection{The study of electron transport within the family of wide energy gap semiconductors}

Wide energy gap semiconductors, i.e., semiconductors with energy gaps wider than those associated with the more conventional semiconductors, Si and GaAs [165], ${ }^{17}$ offer considerable promise for novel electronic and opto-electronic device applications [2,144,145,166-170]. Owing to the fact that wider energy gap semiconductors tend to possess higher polar optical phonon energies, the saturation electron drift velocities exhibited by these materials tend to be higher [1, 171-173]. In addition, the dielectric constants, both static and high-frequency, associated with the wider energy gap semiconductors tend to be smaller than those associated with the more conventional semiconductors $[8,125]$. Both of these factors favor improved electron device performance [174-176]. An additional benefit of the wide energy gap semiconductors is their great tolerance to high applied electric field strengths, the breakdown field strength of a semiconductor material increasing with the magnitude of its energy gap [177,178]. Finally, the high thermal conductivities associated with some of these materials further adds to their allure [146,179].

While pioneering studies into the material properties of some key wide energy gap semiconductors were pursued during the earlier parts of the $20^{\text {th }}$ Century [180-189], it was really only 50-years later that the material quality approached the levels required for device applications. Accordingly, interest into the family of wide energy gap semiconductors, whose promise had long been recognized albeit unrealized, experienced a resurgence. The earliest recorded studies into the material properties of the III-V nitride semiconductors, GaN, AIN, and InN, were performed in

\footnotetext{
17 Yoder [165] defines a wide energy gap semiconductor as being that possessing an energy gap equal to $2.2 \mathrm{eV}$ or wider.
} 
the 1920s, 1930s and 1940s [40-45]. Unfortunately, the materials available at the time, small crystals and powders [3], were of poor quality. Thus, the III-V nitride semiconductor group remained of widely recognized but unrealized potential for many years. It was only with the advent of modern deposition approaches, such as molecular beam epitaxy and metal-organic chemical vapor deposition, that the material quality exhibited by these materials approached the levels demanded of device applications. For the specific case of $\mathrm{GaN}$, for example, improvements in the deposition process only started in the late 1960s; in 1969, Maruska and Tietjen [180] employed chemical vapor deposition in order to fabricate GaN. Since then, dramatic improvements in the material quality of the III-V nitride semiconductors, GaN, AIN, and InN, have been achieved. As a result, interest into the III-V nitride semiconductors, GaN, AIN, and InN, experienced a renaissance; this intense renewed interest into the III-V nitride semiconductors began in earnest in the early 1990s [3]. The work that arose as a consequence of this renewed interest is responsible for the genesis of the III-V nitride semiconductor industry that exists today. At present, GaN, AIN, and InN-based electronic and opto-electronic devices are widely available, such devices being used for a variety of commercial, industrial, and military applications [148,181,182]. Studies into the material properties of $\mathrm{ZnO}$ also found their genesis in the 1920s and 1930s [183185]. SiC already had a significant following by that time, it being a ubiquitous material in the production of steel by the earliest parts of the $20^{\text {th }}$ Century $[186,187]$. Later, by the 1930 s, it was recognized as a material of interest in its own right $[188,189]$. Other wide energy gap semiconductors were introduced over the span of the $20^{\text {th }}$ Century.

From the perspective of device applications, initial interest into the wide energy gap semiconductors focused on their considerable potential for high-frequency, high-power, hightemperature, and high-radiation device applications, where traditional semiconductors prove inadequate [21,50-56]. The potential of wide energy gap semiconductors for use in optical devices, such as lasers and light-emitting diodes, operating in the blue-to-ultraviolet region of the 
electromagnetic spectrum, a region not traditionally served by opto-electronic devices, further fueled interest into these materials [11,26,57-61,180]. SiC, a column-IV-based compound semiconductor that can crystallize in the form of many polytypes $[190],{ }^{18}$ was one of the first wide energy gap semiconductors to acquire the material quality demanded of commercial and military device applications, and novel devices, fabricated from this material, continue to be devised and fabricated today $[4,21,168,191-220] .{ }^{19}$ Later, interest in the wide energy gap semiconductors broadened to include the family of III-V nitride semiconductors, GaN, AIN, and InN [221-225], ${ }^{20}$ and their alloys $[3-6,10,12,48,83,115,132,133,147,148,180,181,226-263] .{ }^{21}$

18 In principle, SiC can crystallize in the form of an infinite number of polytypes. Thus far, over 250 polytypes of SiC have actually been experimentally observed [190].

19 The more common polytypes of SiC possess wide and indirect energy gaps that range between 2.2 and $3 \mathrm{eV}[14,213,214]$. SiC is also found to exhibit a high breakdown field [216,217], an elevated thermal conductivity [217,218], and favorable electron transport characteristics [220]. This constellation of material properties associated with the various polytypes of $\mathrm{SiC}$, and the recognition of the device opportunities thus engendered, were, in large measure, responsible for igniting interest into this material in the first place.

$20 \mathrm{InN}$, while not a wide energy gap semiconductor in of itself, its room temperature energy gap only being around $0.7 \mathrm{eV}$ [221], is often alloyed with the other III-V nitride semiconductors, and thus, is often considered an honorary member of the wide energy gap semiconductor family [221-225].

${ }^{21}$ Initial interest in the device applications of the III-V nitride semiconductors focused on GaN, the wurtzite phase of this material exhibiting a wide and direct energy gap of around $3.39 \mathrm{eV}$ [180]. Wurtzite GaN also exhibits a high breakdown field [244,245], elevated thermal conductivity [246,247], and superb electron transport characteristics $[115,132,133,227]$. These attributes make GaN ideally suited for both electronic and opto-electronic device applications [248-263]. 
Most recently, $\mathrm{ZnO}$, a II-VI wide energy gap semiconductor, has also become of a focus of the wide energy gap semiconductor community $[110,147,149-151,182,233,235,264-268] .{ }^{22}$ Other wide energy gap semiconductors that have been studied include, but are not limited to, AIP [269,270], boron nitride [271-273], CdS [274-276], diamond [277,278], GaP [279-281], ZnS [282-284], ZnSe [285-287], and ZnTe [288,289].

In this chapter, I present a brief overview into what is currently known about the electron transport within the family of wide energy gap semiconductors, describe how this understanding has evolved into its current form, and present a number of applications for the results presented thus far. For the purposes of this review, we primarily focus on the nature of the electron transport that occurs within the III-V nitride semiconductors, GaN, AIN, and $I n N$, and the II-VI semiconductor, $\mathrm{ZnO}$, the electron transport that occurs within some other wide energy gap semiconductors receiving a brief mention. We start with a survey, describing the evolution of the field. In particular, the sequence of critical developments that have occurred, contributing to our current understanding of the electron transport mechanisms within $\mathrm{GaN}, \mathrm{AIN}, \mathrm{InN}$, and $\mathrm{ZnO}$, is chronicled. Then, some current literature is presented, particular emphasis being placed on the developments that have transpired over the past few years and how such developments continue to shape our understanding of the electron transport mechanisms within $\mathrm{GaN}, \mathrm{AIN}, \mathrm{InN}$, and $\mathrm{ZnO}$. Applications of these results, which include a comparison with the results of experiment and the setting of expectations for device performance, are then discussed. Finally, frontiers for further research and investigation are presented. While the primary focus of this thesis is on the nature

$22 \mathrm{ZnO}$, while currently finding applications as a material for low-field thin-film transistor electron device structures [265] and as a potential material for transparent conducting electrodes [266], also possesses a direct energy gap $[110,267]$ with a magnitude that is very similar to that exhibited by GaN [268]. Thus, it might be expected that, with some further improvements in its material quality, $\mathrm{ZnO}$ may also be employed for some of the device roles currently implemented or envisaged for GaN. 
of the electron transport within the wurtzite and zinc-blende phases of GaN and InN, given that pioneering studies into the nature of the electron transport within the wurtzite phase of AIN were motivated and informed by those that were performed for GaN and InN, and given that many GaN and $\ln \mathrm{N}$ based devices are alloyed with AIN, we would be remiss not to mention electron transport results corresponding to wurtzite AIN within the scope of this thesis. $\mathrm{ZnO}$ results are included in this thesis as there have been some recent developments in understanding the nature of the electron transport within this material. Other wide energy gap semiconductors receive a brief mention.

This chapter is organized in the following manner. In Section 4.2, I present a brief survey, describing the evolution of the field. Then, in Section 4.3, the developments that have occurred over the past few years are highlighted. Finally, some applications of the results are featured in Section 4.4 .

\subsection{The evolution of the field}

The favorable electron transport characteristics of the wide energy gap semiconductor, GaN, has been recognized for a long time now. As early as the 1970s, Littlejohn et al. [142] pointed out that the large polar optical phonon energy characteristic of $\mathrm{GaN}$, in conjunction with its wide conduction band inter-valley energy separation, suggests a high saturation electron drift velocity for this material. As high-frequency electron device performance is, in large measure, determined by this saturation electron drift velocity [1], the recognition of this fact ignited enhanced interest into this material, and the broader class of wide energy gap semiconductors. This enhanced interest, and the developments which have transpired as a result of it, are responsible for the wide energy gap compound semiconductor industry of today. In the subsections associated with this particular section, a sampling of electron transport results, corresponding to each of the materials considered in this analysis, i.e., GaN, AIN, InN, and $\mathrm{ZnO}$, is presented. 
Finally, a brief mention of studies into the nature of the electron transport that occurs within other wide energy gap semiconductors is provided.

\subsubsection{Electron transport within GaN: a review}

In 1975, Littlejohn et al. [142] were the first to report results obtained from semi-classical Monte Carlo simulations of the steady-state electron transport within wurtzite GaN. A one-valley model for the conduction band was adopted for the purposes of their analysis. Steady-state electron transport, for both parabolic and non-parabolic band structures, was considered in their analysis, non-parabolicity being treated through the application of the Kane model [109]. The primary focus of their investigation was the determination of the velocity-field characteristic associated with wurtzite GaN. All donors were assumed to be ionized and the free electron concentration was taken to be equal to the dopant concentration. The scattering mechanisms considered were: (1) ionized impurity, (2) polar optical phonon, (3) piezoelectric, and (4) acoustic deformation potential. For the case of the parabolic band, in the absence of ionized impurities, they found that the electron drift velocity monotonically increases with the applied electric field strength, saturating at a value of around $2.5 \times 10^{7} \mathrm{~cm} / \mathrm{s}$ for the case of high applied electric field strengths. In contrast, for the case of the non-parabolic band, in the absence of ionized impurities, a region of negative differential mobility was found, the electron drift velocity achieving a maximum of about $2 \times 10^{7} \mathrm{~cm} / \mathrm{s}$ at an applied electric field strength of around $100 \mathrm{kV} / \mathrm{cm}$, further increases in the applied electric field strength resulting in a slight decrease in the corresponding electron drift velocity. The role of ionized impurity scattering was also investigated by Littlejohn et al. [142].

In 1993, Gelmont et al. [117] reported on ensemble semi-classical two-valley Monte Carlo simulations of the electron transport within wurtzite GaN, this analysis improving upon the analysis of Littlejohn et al. [142] by incorporating inter-valley scattering into the simulations. They found that the negative differential mobility exhibited by wurtzite GaN is much more pronounced than 
that found by Littlejohn et al. [142], and that inter-valley transitions are responsible for this. For a doping concentration of $10^{17} \mathrm{~cm}^{-3}$, Gelmont et al. [117] demonstrated that the electron drift velocity achieves a peak value of about $2.8 \times 10^{7} \mathrm{~cm} / \mathrm{s}$ at an applied electric field strength of around 140 $\mathrm{kV} / \mathrm{cm}$. The impact of inter-valley transitions on the electron distribution function was also determined and shown to be significant. The impact of doping and compensation on the velocityfield characteristic associated with bulk wurtzite GaN was also examined.

Since these pioneering investigations, ensemble Monte Carlo simulations of the electron transport within GaN have been performed numerous times. In particular, in 1995, Mansour et al. [290] reported the use of semi-classical Monte Carlo simulations in order to determine how the crystal temperature influences the velocity-field characteristic associated with wurtzite GaN. Also in 1995, Kolník et al. [63] reported on employing full-band Monte Carlo simulations of the electron transport within wurtzite GaN and zinc-blende GaN, finding that zinc-blende GaN exhibits a much higher low-field electron drift mobility than wurtzite GaN. The peak electron drift velocity corresponding to zinc-blende GaN was found to be only marginally greater than that exhibited by wurtzite GaN. In 1997, Bhapkar and Shur [122] reported on employing ensemble semi-classical three-valley Monte Carlo simulations of the electron transport within bulk and confined wurtzite GaN. Their simulations demonstrated that the two-dimensional electron gas within a confined wurtzite GaN structure will exhibit a higher low-field electron drift mobility than bulk wurtzite GaN, by almost an order of magnitude, this being in agreement with experiment [291]. In 1998, Albrecht et al. [292] reported on employing ensemble semi-classical five-valley Monte Carlo simulations of the electron transport within wurtzite GaN, with the aim of determining elementary analytical expressions for a number of the electron transport metrics corresponding to wurtzite GaN, for the purposes of device modeling.

The first known study of transient electron transport within GaN was that performed by Foutz et al. [64], reported in 1997. In this study, ensemble semi-classical three-valley Monte Carlo 
simulations were employed in order to determine how the electrons within wurtzite and zincblende GaN, initially in thermal equilibrium, respond to the sudden application of a constant electric field. The velocity overshoot that occurs within these materials was examined. It was found that the electron drift velocities that occur within the zinc-blende phase of GaN are slightly greater than those exhibited by the wurtzite phase owing to the slightly higher steady-state electron drift velocity exhibited by the zinc-blende phase of GaN. A comparison with the transient electron transport which occurs within GaAs was made. Using the results of this analysis, a determination of the minimum transit-time, as a function of the distance displaced since the application of the applied electric field, was performed for all three materials considered in their study, i.e., wurtzite GaN, zinc-blende GaN, and zinc-blende GaAs. For distances in excess of 0.1 $\mu \mathrm{m}$, both phases of GaN were shown to exhibit superior performance, i.e., reduced transit-time, when contrasted with that associated with zinc-blende GaAs.

A more general analysis, in which transient electron transport within wurtzite GaN was contrasted with that corresponding to two other III-V nitride semiconductors, i.e., the wurtzite phases of AIN and InN, and zinc-blende GaAs, was then performed by Foutz et al. [132] and reported in 1999. As with their previous study, Foutz et al. [132] determined how electrons, initially in thermal equilibrium, respond to the sudden application of a constant electric field. For all the semiconductors considered, it was found that the electron drift velocity overshoot only occurs when the applied electric field strength exceeds a certain critical applied electric field strength unique to each material. The critical applied electric field strength was found to be $140 \mathrm{kV} / \mathrm{cm}$ for the case of wurtzite GaN, this corresponding to the peak field strength for this material, i.e., the electric field strength at which the peak electron drift velocity in the velocity-field characteristic associated with this material occurs; recall Figure 3.1 of this thesis. This observation was also found to apply for the other semiconductors considered in their analysis. A comparison with the results of experiment was also performed. 
In addition to Monte Carlo simulations of the electron transport within these materials, a number of other types of electron transport studies have been performed. Reports on experimental measurements of the Hall mobility associated with wurtzite GaN are numerous, and include those made by Yoshida et al. [293], Khan et al. [291], Nakayama et al. [294], and Hurni et al. [295]. Experimental measurements of the velocity-field characteristics and of the transient electron transport response of wurtzite GaN have been reported on by Wraback et al. [171,172]; further details, concerning these experimental measurements, were presented by Wraback et al. [173].

Theoretical investigations into the electron transport processes within these materials, in addition to the aforementioned Monte Carlo studies, are also numerous. In 1975, for example, Ferry [1] reported on the determination of the velocity-field characteristic associated with wurtzite GaN using a displaced Maxwellian distribution function approach. For high applied electric field strengths, Ferry [1] found that the electron drift velocity associated with wurtzite GaN monotonically increases with the applied electric field strength, i.e., it does not saturate, reaching a value of about $2.5 \times 10^{7} \mathrm{~cm} / \mathrm{s}$ at an applied electric field strength of $300 \mathrm{kV} / \mathrm{cm}$. The device implications of this result were further explored by Das and Ferry [2]. In 1994, Chin et al. [100] reported on a detailed study of the dependence of the low-field electron drift mobilities associated with wurtzite GaN, and two other III-V nitride semiconductors, i.e., the wurtzite phases of AIN and InN, on the crystal temperature and the doping concentration. An analytical expression for the low-field electron drift mobility, $\mu$, determined using a variational principle, was employed for the purposes of this analysis. The results obtained were contrasted with those of experiment. Subsequent mobility studies were reported on in 1996 by Shur et al. [101] and in 1997 by Look et al. [102]. Then, in 1998, Weimann et al. [296] reported on a model for the determination of how the scattering of electrons by the threading dislocation lines within wurtzite GaN influences the low-field electron drift mobility. They demonstrated why the experimentally measured low-field 
electron drift mobility associated with this material is much lower than that predicted from Monte Carlo analyses, threading dislocations not being taken into account in the Monte Carlo simulations of the electron transport within wurtzite GaN.

While the negative differential mobility exhibited by the velocity-field characteristics associated with GaN is widely attributed to inter-valley transitions, and while direct experimental evidence confirming this has been presented [297], Krishnamurthy et al. [298] suggest that instead the inflection points in the bands, located in the vicinity of the $\Gamma$ valley, are primarily responsible for the negative differential mobility exhibited by wurtzite GaN. The relative importance of these two mechanisms, i.e., inter-valley transitions and inflection point considerations, were evaluated by Krishnamurthy et al. [298], both for the case of wurtzite GaN and an alloy of GaN with another III-V nitride semiconductor.

On the theoretical front, there have been a number of more recent developments. Hotelectron energy relaxation times within wurtzite GaN were studied by Matulionis et al. [299], and reported on in 2002. Bulutay et al. [300] studied the electron momentum and energy relaxation times within wurtzite GaN, and reported the results of their study in 2003. It is particularly interesting to note that the arguments of Bulutay et al. [300] add considerable credence to the earlier inflection point argument of Krishnamurthy et al. [298]. In 2004, Brazis and Raguotis [301] reported on the results of a Monte Carlo study involving additional phonon modes and a smaller conduction band inter-valley energy separation for the case of wurtzite GaN. Their results were found to be much closer to the experimental results of Wraback et al. [171] than those found previously.

The influence of hot-phonons on the electron transport mechanisms within wurtzite GaN, an effect not considered in our simulations of the electron transport within these materials, i.e., we assumed steady-state phonon populations, has been the focus of considerable investigation. 
In particular, in 2004 itself, Gökden [302], Ridley et al. [303], and Silva and Nascimento [304], to name just three, presented results related to this research focus. These results suggest that hotphonon effects play a role in influencing the nature of the electron transport within wurtzite GaN. In particular, Ridley et al. [303] point out that the saturation electron drift velocity and the peak field strength are both influenced by hot-phonon effects; it should be noted, however, that Ridley et al. [303] neglect conduction band inter-valley transitions in their analysis, their analysis challenging the conventional belief that the negative differential mobility exhibited by the velocityfield characteristics associated with wurtzite $\mathrm{GaN}$ is attributable to transitions into the upper energy conduction band valleys. More recent research into this topic was pursued by Martininez et al. [305], Tas et al. [306], Ramonas et al. [307], and Matulionis et al. [308]. Research into the role that hot-phonons play in influencing the electron transport mechanisms within wurtzite GaN seems likely to continue into the foreseeable future.

Research into how the electron transport within wurtzite GaN influences the performance of GaN-based devices is ongoing. In 2004, Matulionis and Liberis [309] reported on the role that hot-phonons play in determining the microwave noise within AIGaN/GaN channels. In 2005, Ramonas et al. [310] further developed this analysis, focusing on how hot-phonon effects influence power dissipation within AIGaN/GaN channels. The high-field electron transport within AIGaN/GaN heterostructures was examined and reported on in 2005 by Barker et al. [311] and Ardaravičius et al. [312]. A numerical simulation of the current-voltage characteristics of AIGaN/GaN high electron mobility transistors at high temperatures was performed by Chang et al. [313] and reported on in 2005. Other device modeling work, involving Monte Carlo simulations of the electron transport within wurtzite GaN, was reported on in 2005 by Yamakawa et al. [314] and Reklaitis and Reggiani [315], and many others since then. 
Finally, the determination of the electron drift velocity from experimental measurements of the unity gain cut-off frequency, $f_{t}$, has been pursued by a number of researchers. The key challenge in these analyses is the de-embedding of the parasitics from the experimental measurements so that the true intrinsic saturation electron drift velocity may be obtained. Following in the tradition of Eastman et al. [316], in 2005 Oxley and Uren [317] found a saturation electron drift velocity of about $1.1 \times 10^{7} \mathrm{~cm} / \mathrm{s}$ for the case of wurtzite $\mathrm{GaN}$. The role of self-heating was also probed by Oxley and Uren [317] and shown to be relatively insignificant. It should be noted, however, that a completely satisfactory explanation for the discrepancy between these experimental results and those of the Monte Carlo simulations has yet to be provided.

\subsubsection{Electron transport within AIN: a review}

In 1998, O'Leary et al. [118] were the first to report results obtained from semi-classical Monte Carlo simulations of the steady-state electron transport within wurtzite AIN. A three-valley model for the conduction band was adopted for the purposes of their analysis. Steady-state electron transport was considered in their analysis, non-parabolicity being treated through the application of the Kane model [109]. The primary focus of their investigation was the determination of the velocity-field characteristic associated with wurtzite AIN, and an assessment as to how this characteristic varies subject to changes in the crystal temperature, the doping concentration, and the piezoelectric constant, $\mathrm{e}_{14}$, as defined earlier in the text. All donors were assumed to be ionized and the free electron concentration was taken to be equal to the dopant concentration. The scattering mechanisms considered were: (1) ionized impurity, (2) polar optical phonon, (3) piezoelectric, and (4) acoustic deformation potential. For the crystal temperature set to $300 \mathrm{~K}$ and the doping concentration being set to $10^{17} \mathrm{~cm}^{-3}$, it was found that initially the electron drift velocity monotonically increases with the applied electric field strength, reaching a maximum of about 1.7 $\times 10^{7} \mathrm{~cm} / \mathrm{s}$ when the applied electric field strength is around $450 \mathrm{kV} / \mathrm{cm}$. Further increases in the applied electric field strength were found to result in a decrease in the corresponding electron drift 
velocity, the saturation high-field electron drift velocity associated with this material being found to be about $1.4 \times 10^{7} \mathrm{~cm} / \mathrm{s}$.

In 1999, Albrecht et al. [110] reported on ensemble semi-classical five-valley Monte Carlo simulations of the electron transport within wurtzite AIN. All the scattering mechanisms considered in the analysis of O'Leary et al. [118] were considered by Albrecht et al. [110], with the exception of piezoelectric scattering. Degeneracy effects, considered by O'Leary et al. [118] using the rejection technique of Lugli and Ferry [128], were neglected by Albrecht et al. [110]. Minor differences in the material and band structural parameters are found between those of O'Leary et al. [118] and Albrecht et al. [110]. As a consequence, Albrecht et al. [110] obtain results that are slightly different from those of O'Leary et al. [118]. In particular, for the crystal temperature set to $300 \mathrm{~K}$ and the doping concentration set to $10^{17} \mathrm{~cm}^{-3}$, Albrecht et al. [110] find that the electron drift velocity associated with wurtzite AIN achieves a peak value of about $2.4 \times 10^{7} \mathrm{~cm} / \mathrm{s}$ when the applied electric field strength is around $340 \mathrm{kV} / \mathrm{cm}$. The high-field saturation electron drift velocity associated with the analysis of Albrecht et al. [110] remains unknown as it lies beyond the scale of the velocity-field characteristic depicted in their paper and was not indicated in the manuscript itself.

Since these pioneering investigations, ensemble Monte Carlo simulations of the electron transport within AIN have been performed a number of times. In particular, as has already been indicated, the transient electron transport that occurs within AIN was studied by Foutz et al. [132] in 1999. Then, in 2001, Farahmand et al. [318] presented Monte Carlo electron transport simulation results corresponding to alloys of the semiconductor $A l_{x} \mathrm{Ga}_{1-x} \mathrm{~N}$, AIN being a special case of this alloy, corresponding to $x$ being set to unity. Also in 2001, Osman [319] studied how hot phonons shape the electron transport characteristics associated with this material. In 2002, Li et al. [320] devised a model for GaN/AIGaN-based high-electron mobility transistors, Monte Carlo simulations of the electron transport within these materials forming the basis upon which their an- 
alysis was built. In 2003, Sevik and Bulutay [321] studied hot-electron effects within GaN/AIGaNbased devices using Monte Carlo simulations of the electron transport, a portion of this electron transport corresponding to the specific case of the electron transport within AIN itself. Other research results, related to the electron transport within AIN, or alloys with AIN, have also been reported, and are further discussed in the scientific literature [100,322-324].

\subsubsection{Electron transport within InN: a review}

In 1998, O'Leary et al. [130] were the first to report results obtained from semi-classical Monte Carlo simulations of the steady-state electron transport within the wurtzite phase of $\ln N$ [136]. A three-valley model for the conduction band was adopted for the purposes of their analysis. Steady-state electron transport was considered in their analysis, non-parabolicity being treated through the application of the Kane model [109]. The primary focus of their investigation was the determination of the velocity-field characteristic associated with wurtzite $\operatorname{InN}$, and an assessment as to how it varies subject to changes in the crystal temperature and the doping concentration. All donors were assumed to be ionized and the free electron concentration was taken to be equal to the dopant concentration. The scattering mechanisms considered were: (1) ionized impurity, (2) polar optical phonon, (3) piezoelectric, and (4) acoustic deformation potential. For the crystal temperature set to $300 \mathrm{~K}$ and the doping concentration set to $10^{17} \mathrm{~cm}^{-3}$, it was found that initially the electron drift velocity monotonically increases with the applied electric field strength, reaching a maximum of about $4.3 \times 10^{7} \mathrm{~cm} / \mathrm{s}$ when the applied electric field strength is around $65 \mathrm{kV} / \mathrm{cm}$. Further increases in the applied electric field strength were found to result in a decrease in the corresponding electron drift velocity, the saturation high-field electron drift velocity associated with this material being found to be about $2.5 \times 10^{7} \mathrm{~cm} / \mathrm{s}$. 
All of the scattering mechanisms considered in the analysis of O'Leary et al. [130] were considered by Bellotti et al. [325] except for impact ionization which was included in the analysis of Bellotti et al. [325]; O'Leary et al. [130] neglect impact ionization. Minor differences in the material and band structural parameters are found between those of O'Leary et al. [130] and Bellotti et al. [325]. As a consequence, Bellotti et al. [325] obtained results that are slightly different from those of O'Leary et al. [130]. In particular, for the crystal temperature set to $300 \mathrm{~K}$ and the doping concentration set to $10^{17} \mathrm{~cm}^{-3}$, Bellotti et al. [325] find that the electron drift velocity associated with wurtzite InN achieves a peak value of about $4.2 \times 10^{7} \mathrm{~cm} / \mathrm{s}$ when the applied electric field strength is around $65 \mathrm{kV} / \mathrm{cm}$; this value corresponds to the full-band simulation, the five-valley simulation resulting in a peak electron drift velocity value of about $3.9 \times 10^{7} \mathrm{~cm} / \mathrm{s}$ when the applied electric field strength is around $60 \mathrm{kV} / \mathrm{cm}$. The saturation electron drift velocity associated with the analysis of Bellotti et al. [325] remains unknown as it lies beyond the scale of the velocity-field characteristic depicted in their paper and was not indicated in the manuscript itself.

Interest in the wurtzite phase of InN was greatly increased in 2002 with the realization that the energy gap of this material is much smaller than had been initially believed [221, 326-328]. ${ }^{23}$

23 In 1986, Tansley and Foley [326] measured the spectral dependence of the optical absorption coefficient associated with wurtzite $\ln N$ and determined that the energy gap associated with this material is around $1.89 \mathrm{eV}$. This value became the de facto standard for the field until 2002, when Wu et al. [221] demonstrated, using higher quality forms of wurtzite $\operatorname{lnN}$, that the energy gap associated with this material is actually around $0.7 \mathrm{eV}$. Other experimental measurements confirmed the narrower energy gap value suggested by Wu et al. $[221,327,328]$. This revised value for the wurtzite $\ln N$ energy gap is now widely accepted by the semiconductor materials community. 
This suggests that the family of III-V nitride semiconductors, and their alloys, form a continuous direct-gap material alloy system, ranging from the infra-red to the ultra-violet regions of the electromagnetic spectrum, opening up opportunities for novel device applications not otherwise obtainable using other material systems. This, of course, has generated interest in understanding the material properties associated with InN. As a result, a number of studies have been performed on the electron transport within this material since 2002.

In 2005, O'Leary et al. [138] performed Monte Carlo simulations of the electron transport that occurs within wurtzite InN using a revised set of material and band structural parameters taking into account this new value of the energy gap associated with this material. Then, building upon this analysis, in 2006, O'Leary et al. [139] employed a Monte Carlo simulation approach in order to determine upper-bounds on the frequency response of wurtzite InN-based electron devices. Also in 2006, Polyakov et al. [329] employed Monte Carlo electron transport simulations in order to understand the dependence of the low-field electron drift mobility on the ionized impurity concentration. Later, in 2007, Yarar [330] employed Monte Carlo electron transport simulations in order to characterize the nature of the transient electron transport within this material. In 2009, Polyakov et al. [94] employed a Monte Carlo electron transport simulation approach in order to contrast the electron transport within the wurtzite and zinc-blende phases of $\ln N$. Other research results, related to the electron transport within $\ln N$, or alloys with $\ln N$, have also been reported, and are further discussed in the scientific literature [100,331].

\subsubsection{Electron transport within other wide energy gap semiconductors: a brief mention}

Analyses into the nature of the electron transport that occurs within other wide energy gap semiconductors have also been pursued. In particular, electron transport results, corresponding to the cases of diamond [332,333], GaP [334-336], SiC [220,337-344], ZnO [345,346], ZnS [347], 
and other wide energy gap semiconductors of interest [1], have been reported. Further details, regarding these studies, are available in the relevant scientific literature.

\subsection{Recent developments}

Over the past few years, there have been a number of developments that have occurred that have further enriched our understanding of the electron transport within wurtzite GaN. In 2011, Ilgaz et al. [348] studied the energy relaxation of hot electrons within AIGaN/GaN/GaN heterostructures. Then, in 2012, Naylor et al. [349] examined the steady-state and transient electron transport that occurs within bulk wurtzite GaN using an analytical band-structure that more accurately reflects the nature of the actual band-structure. In 2012, Naylor et al. [350] also examined the electron transport that occurs within dilute $\mathrm{GaN}_{x} \mathrm{As}_{1-\mathrm{x}}$ samples. In 2013, Bellotti et al. [351] employed a full-band model in order to determine the velocity-field characteristics associated with AIGaN alloys. Also in 2013, Dasgupta et al. [352] estimated the hot electron relaxation time associated with wurtzite GaN using a series of electrical measurements. In 2013, Zhang et al. [353] determined the hot-electron relaxation time within lattice-matched InAIN/AIN/GaN heterostructures. The potential for electron device structures was then explored. In 2015, Freedsman et al. [354] examined the enhanced two-dimensional electron gas transport characteristics of $\mathrm{Al}_{2} \mathrm{O}_{3} / \mathrm{AllnNGaN}$ metal-oxide-semiconductor high-electron-mobility transistors that were deposited on Si substrates. Also in 2015, Kourdi et al. [355] demonstrated that through device optimization and control over the distribution of dopants, it is possible to minimize the side effects associated with InAIN/GaN transistors. Then, in 2016, Siddiqua et al. [89] examined, in detail, the nature of the electron transport within the zinc-blende phase of GaN [69-80]. Recent further work on the nature of the electron transport that occurs within GaN has been reported in the literature [356-364]. Clearly, the study of electron transport within GaN remains an area of active inquiry. 
Studies into the electron transport that occurs within InN have also been performed in more recent years. In 2010, O'Leary et al. [365] presented a detailed examination of the sensitivity of the electron transport characteristics associated with wurtzite $\ln N$ on the crystal temperature, the doping concentration, and the non-parabolicity coefficient associated with the lowest energy conduction band valley. In 2011, Baghani and O'Leary [366] determined the dependence of the low-field mobility associated with wurtzite InN on the threading-line concentration. Then, in 2013 , Hadi et al. [134] contrasted the electron transport characteristics associated with wurtzite InN with those associated with zinc-blende $\operatorname{lnN}[81-84]$. The dependence of the inter-valley scattering rate on the non-parabolicity coefficient associated with lowest energy conduction band valley associated with zinc-blende InN was examined by Hadi et al. [367] in 2014. Finally, in 2015, Siddiqua et al. [157] further examined the contrast between the nature of the electron transport with these two phases of InN. Recent further work is found in the scientific literature [90-93]. Clearly, electron transport within $\mathrm{InN}$ remains a field of current and active interest.

\subsection{Applications}

Two important applications for the electron transport results presented within the scope of this thesis can be foreseen. First, the results can be used for materials characterization purposes, providing an interpretational framework for the processing and accounting of experimentally acquired electron transport results. Second, the results presented may be employed in order to set expectations for device performance, providing a benchmark against which progress in the field may be judged.

Before I begin, it should be noted that the results presented, thus far, have exclusively corresponded to the case of bulk semiconductors, where the crystal lattice is of infinite extent. Real semiconductor devices, of course, exhibit a number of important non-idealities that are expected to play an important role in shaping the overall nature of the electron transport that 
occurs. First, a real semiconductor device has finite dimensions, and boundary effects that occur at the surface of such a device, and at the boundaries between the various layers of materials within a given device structure, are expected to have an impact on the electron transport $[368,369]$. Second, most III-V nitride semiconductor-based devices in production today are fabricated through heteroepitaxy, wherein III-V nitride semiconductors are deposited onto foreign substrates, such as $\mathrm{Si}, \mathrm{SiC}$, and sapphire [370-372]. The inevitable lattice mismatches that occur create large concentrations of threading dislocations running through the device [373,374], these also influencing the character of the electron transport that is exhibited $[296,366]$. The multiple layers of materials that are present within III-V nitride semiconductor-based devices, and the complex geometries often employed in the devices fabricated from these materials, make it difficult to tease out the properties of the individual layers from experimental measurements performed on the overall device structure, although it should be noted that a number of approaches for performing such an analysis have been formulated [375]. Finally, in response to the presence of quantum wells in III-V nitride semiconductor-based device structures coupled with strong polarization effects, two-dimensional electron gases have been found to be present within some of these structures, the electron concentration within such a gas greatly exceeding the corresponding background doping concentration within the spatially confined region where such a gas exists [376-379]. ${ }^{24}$ So care must be exercised in the application of these results to real-world devices.

The low-field electron mobility, $\mu$, is the most readily acquired experimental quantity. Exp-

${ }^{24}$ Quantum confinement effects, and the complications they engender, must be considered in order to properly treat the nature of such a gas. Accordingly, many two-dimensional electron gas analyses are cast within the framework of a simultaneous solution to both Schrödinger's and Poisson's equations. Further details on such matters are adequately addressed in the literature [376-378]. 
erimental studies into the low-field mobility within a number of III-V nitride semiconductor materials are widely available in the scientific literature [102,375,380]. In Figure 4.1, I contrast low-field mobility experimental results corresponding to wurtzite GaN with those determined through Monte Carlo simulations of the electron transport; we focus on the dependence of the low-field mobility on the crystal temperature for the purposes of this particular comparative analysis. The experimental results that are depicted are those of Tokuda et al. [381], the Hall mobility being plotted as a function of the crystal temperature. The Monte Carlo simulation results, however, are acquired from the slope of the velocity-field characteristics in the low-field limit, the obtained drift mobility being plotted as a function of the crystal temperature. For all Monte Carlo simulations of the electron transport, the electron concentration is set to the doping concentration, the doping concentration being set to $10^{17} \mathrm{~cm}^{-3}$. It is noted, for all crystal temperatures considered, that the Monte Carlo obtained drift mobility results exceed those determined through experiment, the higher temperature trends being noted to be quite similar in nature. We suspect that the neglect of threading dislocations in the simulations, combined with the neglect of freeze-out statistics, ${ }^{25}$ account for most of the differences observed. Differences between the Hall mobility and the drift mobility, i.e., the Hall factor deviating from unity, may also contribute to the observed differences, although this effect is believed to be relatively minor [382].

In light of these electron transport results, we would now like to project limits for the expected performance of electron devices fabricated for the different materials. There are two device performance characteristics that we focus our attention on in this analysis: (1) the cut-off frequency, $f_{t}$, and (2) the operating voltage, $v_{o p}$. The cut-off frequency is inversely proportional to

${ }^{25}$ When freeze-out statistics are taken into account, the ionized impurity concentration is not equal to the doping concentration, i.e., not all donors donate and not all acceptors accept. 


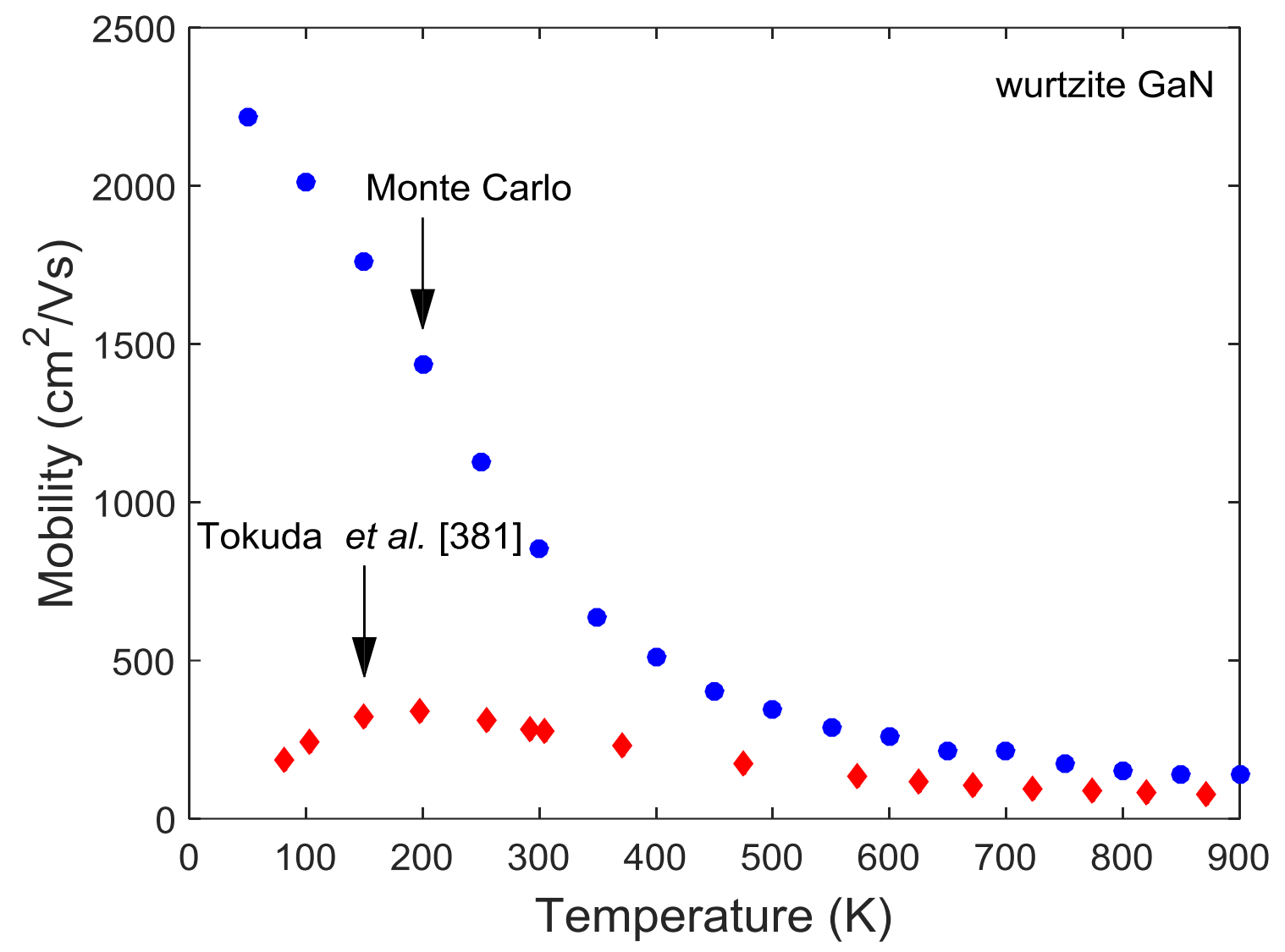

Figure 4.1: The low-field electron mobility of wurtzite GaN plotted as a function of the crystal temperature. The experimental Hall mobility results of Tokuda et al. [381] and electron drift mobility results, acquired from our Monte Carlo simulations of the electron transport, are depicted in this plot. This figure has been modified from @ (c) Siddiqua, P. \& O'Leary, S.K. (2018). Electron transport within the wurtzite and zinc-blende phases of gallium nitride and indium nitride, Journal of Materials Science, 29, 3511-3567. Page 3554. Adapted with permission from publisher. The online version of this figure is depicted in color. 
the electron transit-time, $\tau$, i.e.,

$$
f_{t}=\frac{1}{2 \pi \tau}
$$

The transit-time across such a device may be estimated as

$$
\tau=\frac{L}{v}
$$

where $L$ denotes the corresponding device length-scale and $v$ represents the velocity associated with the electrons.

Setting $v$ to the peak transient electron drift velocity experienced for each material considered, i.e., recall Figure 3.32.b, a prescription for the dependence of $f_{t}$ on the device lengthscale is thus acquired; recall that the transient electron transport responses depicted in Figure 3.32.a are determined assuming a constant and uniform applied electric field strength that is twice the corresponding steady-state peak field strength for each material considered in this analysis, these steady-state peak field strengths being $140,110,30$, and $50 \mathrm{kV} / \mathrm{cm}$ for the cases of wurtzite GaN, zinc-blende GaN, wurtzite InN, and zinc-blende $\operatorname{lnN}$, respectively, as is seen in Figure 3.16. The dependence of the operating voltage, $v_{o p}$, on the device length-scale may be determined by assuming that the applied electric field strength is constant and uniform across the device, i.e.,

$$
v_{o p}=E L,
$$

where $E$ denotes the value of the constant and uniform applied electric field strength. For the purposes of this analysis, we will assume that by setting $E$ to twice the corresponding steadystate peak field strength for each material considered in this analysis, an optimal operating voltage may thus be obtained; recall that this selection of the applied electric field strength was used to 
determine the transient electron transport responses depicted in Figure 3.32.a, these characteristics being used in order to define the dependence of $f_{t}$ on $L$.

In Figure 4.2, the dependence of $f_{t}$ and the optimal operating voltage on the device lengthscale, $L$, are depicted for the four materials considered in this electron transport study, i.e., wurtzite GaN, zinc-blende GaN, wurtzite $\operatorname{lnN}$, and zinc-blende $\operatorname{lnN}$. While it is clear that wurtzite InN's enhanced peak transient electron drift velocity is leading to the best frequency response, i.e., up to $15 \mathrm{THz}$ for electron devices of $10 \mathrm{~nm}$ in dimension, given that it has the lowest steadystate peak field strength, the applied voltage associated with wurtzite $\ln N$ is the lowest amongst the four materials being considered in this analysis. In contrast, it is found that zinc-blende $\ln N$ offers larger optimal operating voltages, almost a factor of two greater than those associated with its wurtzite counterpart. This suggests that perhaps an alloy of these two phases of InN may offer a compromise between the best high-frequency and high-voltage responses $[383,384] .{ }^{26}$ Alternatively, as was recently pointed out by Hadi et al. [367], the non-parabolicity associated with lowest energy conduction band valley could be modified through the introduction of stress into the system, such as that arising from pseudomorphic growth on a heterogeneous substrate. This will lead to a form of zinc-blende $\ln N$ itself with velocity-field characteristics that fall directly between those associated with the bulk wurtzite $\ln N$ and bulk zinc-blende $\ln N$. The practicalities of these proposed scenarios remains unexplored as of the present moment.

26 In the fabrication of III-V nitride semiconductor-based devices, alloying is often employed. Unfortunately, owing to limitations on the scope and scale of this thesis, I opted not to dedicate much of it to alloying. Further details on alloys of the III-V nitride semiconductor group are provided in the scientific literature [383, 384]. 


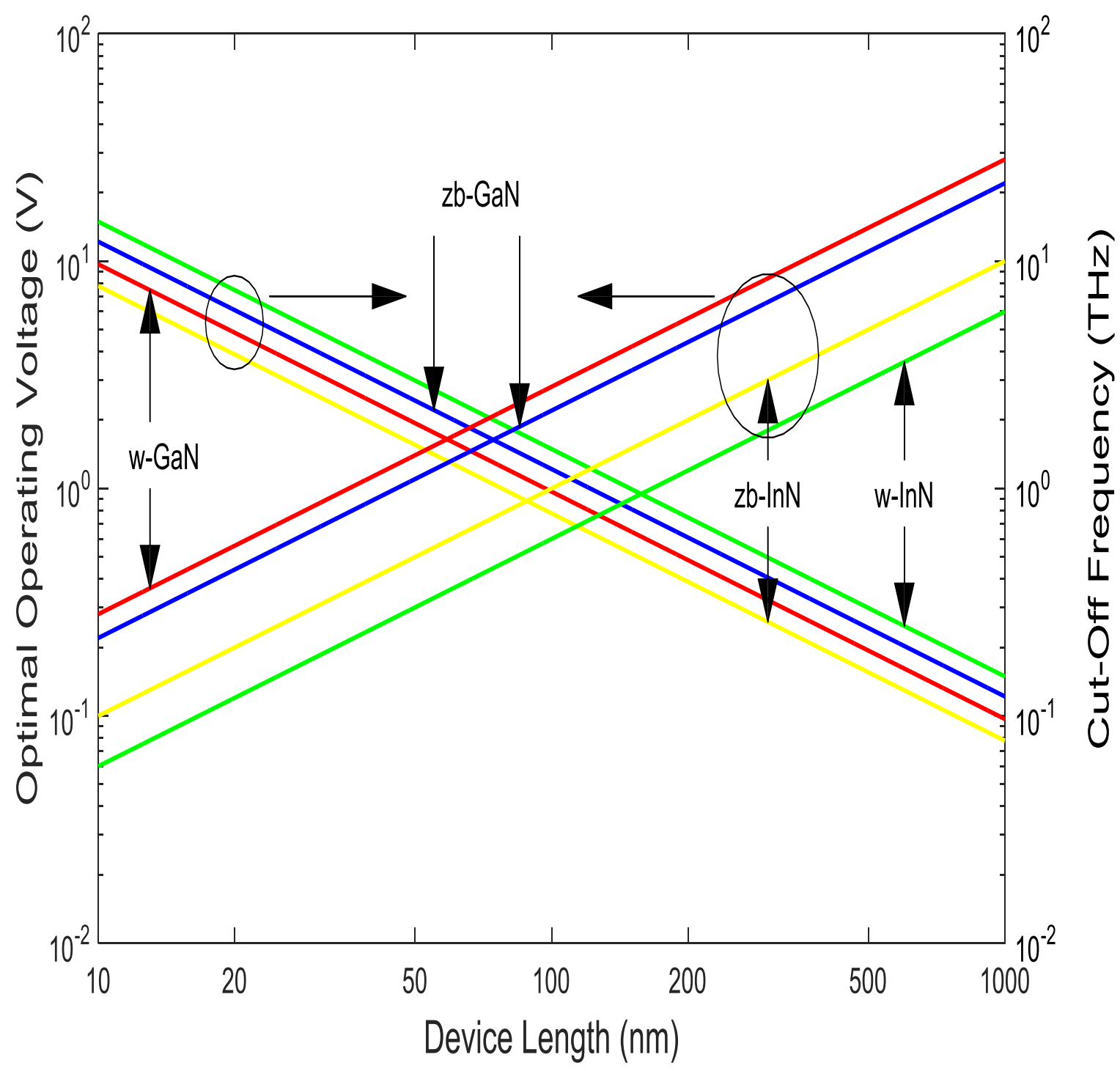

Figure 4.2: The cut-off frequency and the optimal operating voltage plotted as functions of the device length-scale, L, for the cases of wurtzite GaN, zinc-blende GaN, wurtzite InN, and zinc-blende InN. This figure has been modified from ( $)$ Siddiqua, P. \& O'Leary, S.K. (2018). Electron transport within the wurtzite and zinc-blende phases of gallium nitride and indium nitride, Journal of Materials Science, 29, 3511-3567. Page 3555. Adapted with permission from publisher. The online version of this figure is depicted in color. 


\section{Chapter 5: Conclusions}

In this thesis, I reviewed analyses of the electron transport within some III-V nitride semiconductors of current interest. In particular, I have discussed the evolution of the field, surveyed the current literature, and examined some applications for the results presented herein. In order to narrow the scope of this thesis, I focused on the electron transport within the wurtzite and zinc-blende phases of $\mathrm{GaN}$ and InN. Results corresponding to the case of GaAs have also been presented, albeit primarily for benchmarking purposes. Most of my discussion focused upon results obtained from my ensemble semi-classical three-valley Monte Carlo simulations of the electron transport within these materials, my results conforming with state-of-the-art wide energy gap compound semiconductor orthodoxy.

I began this thesis with the Boltzmann transport equation, this equation underlying most analyses of the electron transport within semiconductors. A brief description of my ensemble semi-classical three-valley Monte Carlo simulation approach to solving the Boltzmann transport equation was then provided. The material and band structural parameters, corresponding to the wurtzite and zinc-blende phases of $\mathrm{GaN}$ and $\mathrm{InN}$, and zinc-blende $\mathrm{GaAs}$, were then presented. I then used these parameter selections, in conjunction with my ensemble semi-classical threevalley Monte Carlo simulation approach, in order to determine the nature of the steady-state and transient electron transport within the semiconductors, GaN and InN. Finally, we presented some recent developments on the electron transport within these materials, and examined some applications for the results.

It is clear that our understanding of the semiconductors $\mathrm{GaN}$ and $\operatorname{InN}$, is, at present at least, in a state of flux. A complete understanding of the electron transport mechanisms within these materials has yet to be achieved, and is the subject of intense current research. Most troubling is the discrepancy between the results of experiment and those of simulation. As I 
currently view it, there are a two principle sources of uncertainty in my analysis of the electron transport mechanisms within these materials; (1) uncertainty in the material properties, and (2) uncertainty in the underlying physics. I discuss each of these subsequently.

Uncertainty in the material parameters associated with the semiconductors, GaN and InN, remains a key source of ambiguity in the analysis of the electron transport with these materials [132]. Even for bulk wurtzite GaN, the most well studied of the III-V nitride semiconductors, uncertainty in the band structure remains an issue [385]. Given this uncertainty in the band structures associated with GaN, it is clear that new simulations of the electron transport within this material will have to be performed once researchers have settled on appropriate band structures. I thus view the results presented in Chapter 3 as a baseline, the sensitivity analysis, presented in O'Leary et al. [133], providing some insight into how variations in the band structure itself will impact upon the results. Work on finalizing a set of band structural parameters, suitable for wurtzite GaN, and on performing the corresponding electron transport simulations, is ongoing. Given that the zinc-blende phase of $\mathrm{GaN}$ and the wurtzite and zinc-blende phases of InN are far less studied than the wurtzite phase of $\mathrm{GaN}$, determining a set of band structural parameters, suitable for these other materials, remains an ongoing concern.

Uncertainty in the underlying physics is also considerable. The source of the negative differential mobility, found in the velocity-field characteristics associated with the semiconductors, $\mathrm{GaN}$ and $\mathrm{InN}$, remains a matter to be resolved. The presence of hot-phonons within these materials, and how such phonons impact upon the electron transport mechanisms, remains another point of contention. It is clear that a deeper understanding of these electron transport mechanisms will have to be achieved in order for the next generation of semiconductor devices, based on $\mathrm{GaN}$ or InN, to be properly designed. 
In terms of further exploring the applications of these electron transport results, I would be particularly interested in exploring their capacity in providing the scientific and engineering communities with an analytical framework within which III-V nitride semiconductor based-devices may be engineered and optimized. With respect to maximizing the cut-off frequency, $f_{t}$, an optimization of the device performance may be achieved if one minimizes the transit-time over a given distance. Focusing on transient electron transport results for the case of wurtzite GaN, it can be seen that there is a trade-off between the peak overshoot velocity and the distance taken to achieve steady-state. In particular, recalling Figure 3.27, when the applied electric field strength is set to $560 \mathrm{kV} / \mathrm{cm}$, the peak overshoot velocity is around $9.5 \times 10^{7} \mathrm{~cm} / \mathrm{s}$, while the corresponding steady-state drift velocity, about $1.4 \times 10^{7} \mathrm{~cm} / \mathrm{s}$, is achieved after just $50 \mathrm{~nm}$. However, for the applied field strength of $210 \mathrm{kV} / \mathrm{cm}$, just above the corresponding steady-state peak field strength, the peak overshoot velocity is only around $5.0 \times 10^{7} \mathrm{~cm} / \mathrm{s}$, and it takes longer, about $400 \mathrm{~nm}$, to achieve the corresponding steady-state drift velocity, about $2.2 \times 10^{7} \mathrm{~cm} / \mathrm{s}$. Similar results are noted for the other materials considered in this analysis, i.e., for zinc-blende GaN, wurtzite $\operatorname{lnN}$, and zinc-blende InN. Through a detailed analysis of the transient electron transport responses, the minimum transit-time corresponding to each device length scale, $L$, may be determined.

From this optimization procedure, the minimum transit-time corresponding to each device length-scale, $L$, may be determined. From Eq. (4.7), I am therefore able to plot the dependence of the upper bound for the cut-off frequency as a function of the device length-scale, $L$. In Figure 5.1 [386-393], I plot this dependence for the specific case of wurtzite GaN, these results being from Figure 7 of Foutz et al. [131], this analysis being pursued by Foutz et al. [132]. It should be noted that the result depicted here constitutes an improvement over the steady-state projections for device performance seen previously in Figure 4.2. As a benchmark, I also plot results acquired from the conjuncture of Eqs. (4.6) and (4.7) for the specific case of $v$ being set to the peak steadystate electron drift velocity, i.e., around $2.9 \times 10^{7} \mathrm{~cm} / \mathrm{s}$, for the case of wurtzite GaN (recall Figure 


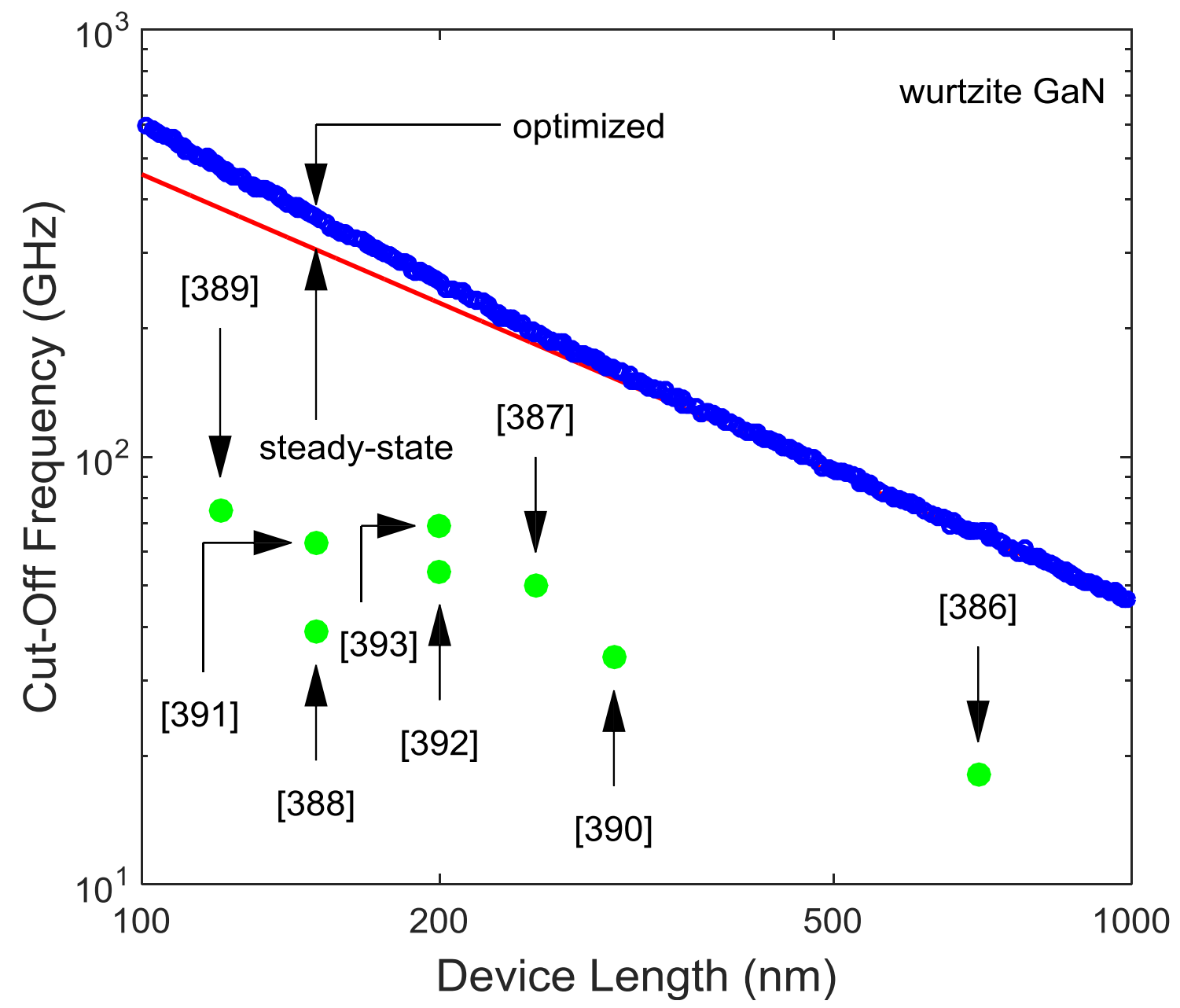

Figure 5.1: The cut-off frequency plotted as a function of the device length-scale, $L$, for the case of wurtzite GaN. The optimized result is depicted with the open blue points. The result obtained assuming the steadystate peak velocity, i.e., around $2.9 \times 10^{7} \mathrm{~cm} / \mathrm{s}$, is depicted with the solid red line. Representative device results, corresponding to the experimental work of Johnson et al. [386], Ducatteau et al. [387], Arulkumaran et al. [388], Medjdoub et al. [389], Arulkumaran et al. [390], Ranjan et al. [391], Huang et al. [392], and Yang et al. [393], are depicted with the solid green points depicted on the figure. This figure has been modified from (C Siddiqua, P. \& O'Leary, S.K. (2018). Electron transport within the wurtzite and zinc-blende phases of gallium nitride and indium nitride, Journal of Materials Science, 29, 3511-3567. Page 3556. Adapted with permission from publisher. The online version of this figure is depicted in color. 
3.16), noting that we would expect optimized transient results to converge with peak steady-state results in the long device length-scale limit. Observing Figure 5.1, I note that the optimized result places an upper bound on the steady-state result, and that this upper bound becomes very tight as the device length-scale becomes large. This comports with my intuition that in the limit that the device length-scale becomes large that steady-state effects will dominate the electron transport characteristics. A few representative experimental results are also depicted in Figure 5.1 [386393]. It is seen that, in all cases, the optimized results place an upper-limit on these experimental electron transport results, as would be expected. Such an analysis performed for the other materials considered in this analysis, i.e., zinc-blende $\mathrm{GaN}$ and the wurtzite and zinc-blende phases of $\operatorname{lnN}$, will provide the community with a benchmark against which progress may be judged. This research will have to be performed in the future. 


\section{References}

1. D.K. Ferry, Phys. Rev. B 12, 2361 (1975)

2. P. Das, D.K. Ferry, Solid-State Electron. 19, 851 (1976)

3. S. Strite, H. Morkoç, J. Vac. Sci. Technol. B 10, 1237 (1992)

4. H. Morkoç, S. Strite, G.B. Gao, M.E. Lin, B. Sverdlov, M. Burns, J. Appl. Phys. 76, 1363 (1994)

5. S.N. Mohammad, H. Morkoç, Prog. Quantum Electron. 20, 361 (1996)

6. S.J. Pearton, J.C. Zolper, R.J. Shul, F. Ren, J. Appl. Phys. 86, 1 (1999)

7. S.C. Jain, M. Willander, J. Narayan, R. Van Overstraeten, J. Appl. Phys. 87, 965 (2000)

8. M.E. Levinshtein, S.L. Rumyantsev, M.S. Shur (eds.), Properties of Advanced Semiconductor Materials: GaN, AIN, InN, BN, SiC, SiGe (Wiley, New York, 2001)

9. A.G. Bhuiyan, A. Hashimoto, A. Yamamoto, J. Appl. Phys. 94, 2779 (2003)

10. M.S. Shur, R.F. Davis (eds.), GaN-Based Materials and Devices: Growth, Fabrication, Characterization and Performance (World Scientific, River Edge, 2004)

11. J. Wu, J. Appl. Phys. 106, 011101 (2009)

12. S.J. Pearton, C.R. Abernathy, F. Ren, Gallium Nitride Processing for Electronics, Sensors and Spintronics (Springer, New York, 2010)

13. E.A. Jones, F. Wang, D. Costinett, IEEE J. Emerg. Sel. Topics Power Electron. 4, 707 (2016)

14. G. Meneghesso, M. Meneghini, I. Rossetto, D. Bisi, S. Stoffels, M. Van Hove, S. Decoutere, E. Zanoni, Semicond. Sci. Technol. 31, 093004 (2016)

15. N. Otsuka, S. Nagai, H. Ishida, Y. Uemoto, T. Ueda, T. Tanaka, D. Ueda, ECS Trans. 41, 51 (2011)

16. J.L. Hudgins, R.W. de Doncker, IEEE Ind. Appl. Mag. 18, 18 (2012)

17. A. Dobrinsky, G. Simin, R. Gaska, M. Shur, ECS Trans. 58, 129 (2013)

18. M.H. Wong, S. Keller, Nidhi, S. Dasgupta, D.J. Denninghoff, S. Kolluri, D.F. Brown, J. Lu, N.A. Fichtenbaum, E. Ahmadi, U. Singisetti, A. Chini, S. Rajan, S.P. DenBaars, J.S. Speck, U.K. Mishra, Semicond. Sci. Technol. 28, 074009 (2013) 
19. S. Faramehr, K. Kalna, P. Igić, Semicond. Sci. Technol. 29, 115020 (2014)

20. P. Fay, Y. Xie, Y. Zhao, Z. Jiang, S. Rahman, H. Xing, B. Sensale- Rodriguez, L. Liu, Proc. SPIE 9199, 91990L (2014)

21. J. Millán, P. Godignon, X. Perpiñà, A. Pérez-Tomás, J. Rebollo, IEEE Trans. Power Electron. $29,2155(2014)$

22. T. Ueda, M. Ishida, T. Tanaka, D. Ueda, Jpn. J. Appl. Phys. 53, 100214 (2014).

23. B.K. Jebalin, A.S. Rekh, P. Prajoon, D. Godwinraj, N.M. Kumar, D. Nirmal, Superlattices Microstruct. 78, $210(2015)$

24. F.A. Maier, D. Krausse, D. Gruner, R. Reiner, P. Waltereit, R. Quay, O. Ambacher, in IEEE Compound Semiconductor Integrated Circuit Symposium (2016), p. 1

25. M. Kuzuhara, J.T. Asubar, H. Tokuda, Jpn. J. Appl. Phys. 55, 070101 (2016)

26. Y.-S. Park, Proc. SPIE 4413, 282 (2001)

27. M. Yang, B. Chang, G. Hao, H. Wang, M. Wang, Optik 126, 3357 (2015)

28. M.S. Shur, M.A. Khan, Proc. SPIE 2397, 294 (1995)

29. M.A. Khan, M.S. Shur, Q. Chen, Electron. Lett. 31, 2130 (1995)

30. J.A. McDonald, R. Szweda, III-Vs Rev. 10, 18 (1997)

31. S. Nakamura, Proc. SPIE 3283, 2 (1998)

32. J. Piprek, R.K. Sink, M.A. Hansen, J.E. Bowers, S.P. DenBaars, Proc. SPIE 3944, 28 (2000)

33. N. Stath, V. Härle, J. Wagner, Mater. Sci. Eng. B 80, 224 (2001)

34. R. Szweda, III-Vs Rev. 15, 40 (2002)

35. M. Meneghini, L.-R. Trevisanello, G. Meneghesso, E. Zanoni, IEEE Trans. Device Mater. Reliab. 8, 323 (2008)

36. M.P. Ulmer, Proc. SPIE 7222, 722210 (2009)

37. S. Mokkapati, C. Jagadish, Mater. Today 12, 22 (2009)

38. N. Trivellin, M. Meneghini, E. Zanoni, K. Orita, M. Yuri, T. Tanaka, D. Ueda, G. Meneghesso, in IEEE International Reliability Physics Symposium Proceedings (2010), p. 1 
39. M. Beeler, E. Trichas, E. Monroy, Semicond. Sci. Technol. 28, 074022 (2013)

40. H. Ott, Zeitschr. Physik 22, 201 (1924)

41. H.J. Krase, J.G. Thompson, J.Y. Yee, Ind. Engg. Chem. 18, 1287 (1926)

42. E. Tiede, M. Thimann, K. Sensse, Chem. Berichte 61, 1568 (1928)

43. W.C. Johnson, J.B. Parsons, M.C. Crew, J. Phys. Chem. 36, 2651 (1932)

44. R. Juza, H. Hahn, Zeitschr. Anorgan. Allgem. Chem. 239, 282 (1938)

45. H. Hahn, R. Juza, Zeitschr. Anorgan. Allgem. Chem. 244, 111 (1940)

46. M.A.L. Johnson, N.A. El-Masry Jr., J.W. Cook Jr., J.F. Schetzina, Mater. Sci. Forum 264-268, 1161 (1998)

47. S. Keller, Y.-F. Wu, G. Parish, N. Ziang, J.J. Xu, B.P. Keller, S.P. DenBaars, U.K. Mishra, IEEE Trans. Electron Devices 48, 552 (2001)

48. F. Scholz, Semicond. Sci. Technol. 27, 024002 (2012)

49. T.J. Flack, B.N. Pushpakaran, S.B. Bayne, J. Electron. Mater. 45, 2673 (2016)

50. R.J. Trew, J.-B. Yan, P.M. Mock, Proc. IEEE 79, 598 (1991)

51. J.M. McGarrity, F.B. McLean, W.M. DeLancey, J. Palmour, C. Carter, J. Edmond, R.E. Oakley, IEEE Trans. Nucl. Sci. 39, 1974 (1992)

52. P.L. Dreike, D.M. Fleetwood, D.B. King, D.C. Sprauer, T.E. Zipperian, IEEE Trans. Compon. Packag. Manuf. Technol. A 17, 594 (1994)

53. J.B. Casady, R.W. Johnson, Solid-State Electron. 39, 1409 (1996)

54. J.C. Zolper, IEDM 1999, 389 (1999)

55. J. Millán, IET Circuits Devices Syst. 1, 372 (2007)

56. V.V. Buniatyan, V.M. Aroutiounian, J. Phys D.: Appl. Phys. 40, 6355 (2007)

57. R.N. Bhargava, IEEE Trans. Electron Devices 22, 691 (1975)

58. A.V. Nurmikko, R.L. Gunshor, IEEE J. Quantum Electron. 30, 619 (1994)

59. T. Matsuoka, A. Ohki, T. Ohno, Y. Kawaguchi, J. Cryst. Growth 138, 727 (1994)

60. M.A. Khan, M.S. Shur, Proc. SPIE 3006, 154 (1997) 
61. J. Han, H. Amano, L. Schowalter, Semicond. Sci. Technol. 29, 080301 (2014)

62. C.-Y. Yeh, Z.W. Lu, S. Froyen, A. Zunger, Phys. Rev. B 46, 10086 (1992)

63. J. Kolník, I.H. Oğuzman, K.F. Brennan, R. Wang, P.P. Ruden, Y. Wang, J. Appl. Phys. 78, 1033 (1995)

64. B.E. Foutz, L.F. Eastman, U.V. Bhapkar, M.S. Shur, Appl. Phys. Lett. 70, 2849 (1997)

65. T. Lei, T.D. Moustakas, R.J. Graham, Y. He, S.J. Berkowitz, J. Appl. Phys. 71, 4933 (1992)

66. Z. Sitar, M.J. Paisley, J. Ruan, J.W. Choyke, R.F. Davis, J. Mater. Sci. Lett. 11, 261 (1992)

67. G. Ramírez-Flores, H. Navarro-Contreras, A. Lastras-Martínez, R.C. Powell, J.E. Greene, Phys. Rev. B 50, 8433 (1994)

68. K.H. Ploog, 0. Brandt, B. Yang, H. Yang, A. Trampert, Proc. SPIE 3283, 20 (1998)

69. S.V. Novikov, C.T. Foxon, A.J. Kent, Phys. Status Solidi A 207, 1277 (2010)

70. C. Mietze, M. Landmann, E. Rauls, H. Machhadani, S. Sakr, M. Tchernycheva, F.H. Julien, W.G. Schmidt, K. Lischka, D.J. As, Phys. Rev. B 83, 195301 (2011)

71. H. Vilchis, V.M. Sánchez-R, Mater. Sci. Semicond. Proc. 37, 68 (2015)

72. M.-H. Kim, F.S. Juang, Y.G. Hong, C.W. Tu, S.-J. Park, in MBE 2002-2002 $12^{\text {th }}$ International Conference on Molecular Beam Epitaxy (2002)

73. M. Mizuta, S. Fujieda, Y. Matsumoto, T. Kawamura, Jpn. J. Appl. Phys. 25, L945 (1986)

74. J.I. Pankove, Mater. Res. Soc. Symp. Proc. 97, 409 (1987)

75. S. Strite, J. Ruan, Z. Li, A. Salvador, H. Chen, D.J. Smith, W.J. Choyke, H. Morkoç, J. Vac.

Sci. Technol. B 9, 1924 (1991)

76. H. Liu, A.C. Frenkel, J.G. Kim, R.M. Park, J. Appl. Phys. 74, 6124 (1993)

77. T.S. Cheng, L.C. Jenkins, S.E. Hooper, C.T. Foxon, J.W. Orton, D.E. Lacklison, Appl. Phys. Lett. 66, 1509 (1995)

78. D. Schikora, M. Hankeln, D.J. As, K. Lischka, T. Litz, A. Waag, T. Buhrow, F. Henneberger, Phys. Rev. B 54, R8381 (1996) 
79. J.H. Buß, J. Rudolph, T. Schupp, D.J. As, K. Lischka, D. Hägele, Appl. Phys. Lett. 97, 062101 (2010)

80. A.W. Wood, R.R. Collino, B.L. Cardozo, F. Naab, Y.Q. Wang, R.S. Goldman, J. Appl. Phys. 110, 124307 (2011)

81. D. Chandrasekhar, D.J. Smith, S. Strite, M.E. Lin, H. Morkoç, J. Cryst. Growth 152, 135 (1995) 82. L.C. Jenkins, T.S. Cheng, C.T. Foxon, S.E. Hooper, J.W. Orton, S.V. Novikov, V.V. Tret'yakov, J. Vac. Sci. Technol. B 13, 1585 (1995)

83. S. Strite, M.E. Lin, H. Morkoç, Thin Solid Films 231, 197 (1993)

84. A. Tabata, A.P. Lima, L.K. Teles, L.M.R. Scolfaro, J.R. Leite, V. Lemos, B. Schöttker, T. Frey, D. Schikora, K. Lischka, Appl. Phys. Lett. 74, 362 (1999)

85. A. Yamamoto, Y. Yamauchi, M. Ohkubo, A. Hashimoto, J. Cryst. Growth 174, 641 (1997)

86. V. Fellmann, P. Jaffrennou, D. Sam-Giao, B. Gayral, K. Lorenz, E. Alves, B. Daudin, Jpn. J. Appl. Phys. 50, 031001 (2011)

87. K. Kusakabe, D. Imai, K. Wang, A. Yoshikawa, Phys. Status Solidi C 13, 205 (2016)

88. J. Li, K.B. Nam, K.H. Kim, T.N. Oder, H.J. Jun, J.Y. Lin, H.X. Jiang, Proc. SPIE 4280, 27 (2001)

89. P. Siddiqua, S.K. O’Leary, J. Appl. Phys. 120, 095701 (2016)

90. Z. Lin, H. Wang, Y. Lin, M. Yang, W. Wang, G. Li, J. Phys. D: Appl. Phys. 49, 115112 (2016) 91. S. Wang, H. Liu, Q. Chen, H. Zhang, J. Mater. Sci.: Mater. Electron. 27, 11353 (2016)

92. D. Barettin, M. Auf der Maur, A. di Carlo, A. Pecchia, A.F. Tsatsulnikov, W.V. Lundin, A.V. Sakharov, A.E. Nikolaev, M. Korytov, N. Cherkashin, M.J. Hÿtch, S.Y. Karpov, Nanotechnology 28, $275201(2017)$

93. N.A. Masyukov, A.V. Dmitriev, J. Appl. Phys. 122, 065701 (2017)

94. V.M. Polyakov, F. Schwierz, F. Fuchs, J. Furthmüller, F. Bechstedt, Appl. Phys. Lett. 94, $022102(2009)$

95. B.R. Nag, Electron Transport in Compound Semiconductors (Springer-Verlag, Berlin, 1980) 
96. M. Shur, Physics of Semiconductor Devices (Prentice-Hall, Englewood Cliffs, 1990)

97. U.K. Mishra, J. Singh, Semiconductor Device Physics and Design (Springer, Dordrecht, 2008)

98. N.W. Ashcroft, N.D. Mermin, Solid State Physics (Saunders College, Philadelphia, 1976)

99. C. Kittel, Introduction to Solid State Physics, 8th edn. (Wiley, New York, 2005)

100. V.W.L. Chin, T.L. Tansley, T. Osotchan, J. Appl. Phys. 75, 7365 (1994)

101. M. Shur, B. Gelmont, M.A. Khan, J. Electron. Mater. 25, 777(1996)

102. D.C. Look, J.R. Sizelove, S. Keller, Y.F. Wu, U.K. Mishra, S.P. DenBaars, Solid State Commun. 102, 297 (1997)

103. E.M. Conwell, M.O. Vassell, IEEE Trans. Electron Devices 13, 22 (1966)

104. P.A. Sandborn, A. Rao, P.A. Blakey, IEEE Trans. Electron Devices 36, 1244 (1989)

105. S. Zukotynski, W. Howlett, Solid-State Electron. 21, 35 (1978)

106. D.K. Ferry, C. Jacoboni (eds.), Quantum Transport in Semiconductors (Plenum Press, New York, 1992)

107. A. Papoulis, Probability, Random Variables, and Stochastic Processes, 3rd edn. (McGrawHill, New York, 1991)

108. R.M. Yorston, J. Comput. Phys. 64, 177 (1986)

109. W. Fawcett, A.D. Boardman, S. Swain, J. Phys. Chem. Solids 31, 1963 (1970)

110. J.D. Albrecht, P.P. Ruden, S. Limpijumnong, W.R.L. Lambrecht, K.F. Brennan, J. Appl. Phys. 86, 6864 (1999)

111. B.K. Ridley, Quantum Processes in Semiconductors, 3rd edn. (Oxford, New York, 1993)

112. C. Jacoboni, L. Reggiani, Rev. Mod. Phys. 55, 645 (1983)

113. C. Jacoboni, P. Lugli, The Monte Carlo Method for Semiconductor Device Simulation (Springer-Verlag, New York, 1989)

114. G.U. Jensen, B. Lund, T.A. Fjeldly, M. Shur, Comput. Phys. Commun. 67, 1 (1991)

115. S.K. O'Leary, B.E. Foutz, M.S. Shur, L.F. Eastman, J. Mater. Sci.: Mater. Electron. 17, 87 (2006) 
116. M.A. Littlejohn, J.R. Hauser, T.H. Glisson, J. Appl. Phys. 48, 4587 (1977)

117. B. Gelmont, K. Kim, M. Shur, J. Appl. Phys. 74, 1818 (1993)

118. S.K. O'Leary, B.E. Foutz, M.S. Shur, U.V. Bhapkar, L.F. Eastman, Solid State Commun. 105, $621(1998)$

119. A. Bykhovski, B. Gelmont, M. Shur, A. Khan, J. Appl. Phys. 77, 1616 (1995)

120. A.D. Bykhovski, V.V. Kaminski, M.S. Shur, Q.C. Chen, M.A. Khan, Appl. Phys. Lett. 68, 818 (1996)

121. P. Siddiqua, S.K. O’Leary, J. Appl. Phys. 119, 095104 (2016)

122. U.V. Bhapkar, M.S. Shur, J. Appl. Phys. 82, 1649 (1997)

123. W. R. L. Lambrecht, B. Segall, in Properties of Group III Nitrides, No. 11 EMIS Datareviews Series, Edited by J. H. Edgar (Inspec, London, 1994), Chapter 4

124. J.S. Blakemore, J. Appl. Phys. 53, R123 (1982)

125. S. Adachi, Properties of Group-IV, III-V and II-VI Semiconductors (Wiley, Chichester, 2005) 126. S.M. Sze, K.K. Ng, Physics of Semiconductor Devices, 3rd edn. (Wiley, Hoboken, 2007) 127. M. Shur, S. Rumyantsev, M. Levinshtein (eds.), SiC Materials and Devices, vol. 1 (World Scientific, London, 2006)

128. P. Lugli, D.K. Ferry, IEEE Trans. Electron Devices 32, 2431 (1985)

129. K. Seeger, Semiconductor Physics: An Introduction, 9th edn. (Springer, Berlin, 2004)

130. S.K. O'Leary, B.E. Foutz, M.S. Shur, U.V. Bhapkar, L.F. Eastman, J. Appl. Phys. 83, 826 (1998)

131. B.E. Foutz, S.K. O'Leary, M.S. Shur, L.F. Eastman, Mater. Res. Soc. Symp. Proc. 572, 445 (1999)

132. B.E. Foutz, S.K. O’Leary, M.S. Shur, L.F. Eastman, J. Appl. Phys. 85, 7727 (1999)

133. S.K. O’Leary, B.E. Foutz, M.S. Shur, L.F. Eastman, J. Electron. Mater. 32, 327 (2003)

134. W.A. Hadi, P.K. Guram, M.S. Shur, S.K. O’Leary, J. Appl. Phys. 113, 113709 (2013) 
135. B.E. Foutz, S.K. O’Leary, M.S. Shur, L.F. Eastman, U.V. Bhapkar, Mater. Res. Soc. Symp. Proc. 482, 821 (1998)

136. S.K. O'Leary, B.E. Foutz, M.S. Shur, L.F. Eastman, U.V. Bhapkar, Mater. Res. Soc. Symp. Proc. 482, 845 (1998)

137. B.E. Foutz, S.K. O'Leary, M.S. Shur, L.F. Eastman, Mater. Res. Soc. Symp. Proc. 512, 555 (1998)

138. S.K. O’Leary, B.E. Foutz, M.S. Shur, L.F. Eastman, Appl. Phys. Lett. 87, 222103 (2005)

139. S.K. O’Leary, B.E. Foutz, M.S. Shur, L.F. Eastman, Appl. Phys. Lett. 88, 152113 (2006)

140. W.A. Hadi, R. Cheekoori, M.S. Shur, S.K. O'Leary, J. Mater. Sci.: Mater. Electron. 24, 807 (2013)

141. W.A. Hadi, M.S. Shur, S.K. O’Leary, J. Mater. Sci.: Mater. Electron. 24, 1624 (2013)

142. M.A. Littlejohn, J.R. Hauser, T.H. Glisson, Appl. Phys. Lett. 26, 625 (1975)

143. P. Siddiqua, W.A. Hadi, M.S. Shur, S.K. O'Leary, J. Mater. Sci.: Mater. Electron. 26, 4475 (2015)

144. A. BenMoussa, A. Soltani, U. Schühle, K. Haenen, Y.M. Chong, W.J. Zhang, R. Dahal, J.Y. Lin, H.X. Jiang, H.A. Barkad, B. BenMoussa, D. Bolsee, C. Hermans, U. Kroth, C. Laubis, V. Mortet, J.C. de Jaeger, B. Giordanengo, M. Richter, F. Scholze, J.F. Hochedez, Diamond Relat. Mater. 18, 860 (2009)

145. D.K. Schroder, Int. J. High Speed Electron. Syst. 21, 1250009 (2012)

146. H. Jain, S. Rajawat, P. Agrawal, in Proceedings of IEEE International Conference on Microwave (2008), p. 878

147. H. Morkoç, Proc. IEEE 98, 1113 (2010)

148. S. Nakamura, M.R. Krames, Proc. IEEE 101, 2211 (2013)

149. A. Ashrafi, C. Jagadish, J. Appl. Phys. 102, 071101 (2007)

150. Ü. Özgür, D. Hofstetter, H. Morkoç, Proc. IEEE 98, 1255 (2010)

151. Y.-S. Choi, J.-W. Kang, D.-K. Hwang, S.-J. Park, IEEE Trans. Electron Devices 57, 26 (2010) 
152. L. Sang, M. Liao, M. Sumiya, Sensors 13, 10482 (2013)

153. F. Roccaforte, P. Fiorenza, G. Greco, R.L. Nigro, F. Giannazzo, A. Patti, M. Saggio, Phys. Status Solidi A 211, 2063 (2014)

154. E.P. Carlson, I.C. Kizilyalli, T.D. Heidel, D.W. Cunningham, ECS Trans. 75, 3 (2016)

155. X. Zhao, L. Zhou, Y. Jiang, R. Cui, Y. Li, Y. Zheng, J. Zuo, H. Zhang, Organ. Electron. 37, $85(2016)$

156. R.J. Kaplar, A.A. Allerman, A.M. Armstrong, M.H. Crawford, J.R. Dickerson, A.J. Fischer, A.G. Baca, E.A. Douglas, ECS J. Solid State Sci. Technol. 6, Q3061 (2017)

157. P. Siddiqua, W.A. Hadi, A.K. Salhotra, M.S. Shur, S.K. O'Leary, J. Appl. Phys. 117, 125705 (2015)

158. D. Maier, M. Alomari, N. Grandjean, J.-F. Carlin, M.-A. di Forte-Poisson, C. Dua, A. Chuvilin, D. Troadec, C. Gaquière, U. Kaiser, S.L. Delage, E. Kohn, IEEE Trans. Device Mater. Reliab. 10, $427(2010)$

159. J.G. Ruch, IEEE Trans. Electron Devices 19, 652 (1972)

160. M.S. Shur, L.F. Eastman, IEEE Trans. Electron Devices 26, 1677 (1979)

161. M. Heiblum, M.I. Nathan, D.C. Thomas, C.M. Knoedler, Phys. Rev. Lett. 55, 2200 (1985)

162. A. Palevski, M. Heiblum, C.P. Umbach, C.M. Knoedler, A.N. Broers, R.H. Koch, Phys. Rev. Lett. 62, 1776 (1989)

163. A. Palevski, C.P. Umbach, M. Heiblum, Appl. Phys. Lett. 55, 1421 (1989)

164. A. Yacoby, U. Sivan, C.P. Umbach, J.M. Hong, Phys. Rev. Lett. 66, 1938 (1991)

165. M.N. Yoder, IEEE Trans. Electron Devices 43, 1633 (1996)

166. D. Jones, A.H. Lettington, Solid State Commun. 11, 701 (1972)

167. B.J. Baliga, IEEE Electron Device Lett. 10, 455 (1989)

168. M. Bhatnagar, B.J. Baliga, IEEE Trans. Electron Devices 40, 645 (1993)

169. T.P. Chow, R. Tyagi, IEEE Trans. Electron Devices 41, 1481 (1994) 
170. J. W. Milligan, S. Sheppard, W. Pribble, Y.-F. Wu, St. G. Müller, J. W. Palmour, in Proc. 2007 IEEE Radar Conf., 960 (2007)

171. M. Wraback, H. Shen, J.C. Carrano, T. Li, J.C. Campbell, M.J. Schurman, I.T. Ferguson, Appl. Phys. Lett. 76, 1155 (2000)

172. M. Wraback, H. Shen, J.C. Carrano, C.J. Collins, J.C. Campbell, R.D. Dupuis, M.J. Schurman, I.T. Ferguson, Appl. Phys. Lett. 79, 1303 (2001)

173. M. Wraback, H. Shen, S. Rudin, Proc. SPIE 4646, 117 (2002)

174. E.O. Johnson, Proc. IEEE Int. Conv. Record 13, 27 (1965)

175. E.O. Johnson, RCA Rev. 26, 163 (1965)

176. R.W. Keyes, Proc. IEEE 60, 225 (1972)

177. J.L. Hudgins, G.S. Simin, E. Santi, M.A. Khan, IEEE Trans. Power Electron. 18, 907 (2003)

178. L.-M. Wang, in Proc. IEEE 25th International Conference on Microelectronics (2006), p. 615

179. D. Shaddock, L. Meyer, J. Tucker, S. Dasgupta, R. Fillion, P. Bronecke, L. Yorinks, P. Kraft, in Proceedings of the 19th IEEE Semi.-Therm. Symposium (2003), p. 42

180. H.P. Maruska, J.J. Tietjen, Appl. Phys. Lett. 15, 327 (1969)

181. T. Kachi, IEICE Electron. Express 10, 1 (2013)

182. H. Morkoç, Ü. Özgür, Zinc Oxide: Fundamentals, Materials and Device Technology (Wiley, Weinheim, 2009)

183. G.S. Parks, C.E. Hablutzel, L.E. Webster, J. Am. Chem. Soc. 49, 2792 (1927)

184. G.I. Finch, H. Wilman, J. Chem. Soc. (1934). https://doi. org/10.1039/JR9340000751

185. V.E. Cosslett, Nature 136, 988 (1935)

186. F.J. Tone, Iron Age (1899)

187. J.G. Fitz, A. Francis, Met. Chem. Eng. (1912)

188. H.E. White, J. Am. Ceram. Soc. 12, 252 (1933)

189. N.W. Thibault, Am. Mineral. 29, 327 (1944)

190. A.L. Ortiz, F. Sánchez-Bajo, F.L. Cumbrera, F. Guiberteau, J. Appl. Cryst. 46, 242 (2013) 
191. Y. Kondo, T. Takahashi, K. Ishii, Y. Hayashi, E. Sakuma, S. Misawa, H. Daimon, M. Yamanaka, S. Yoshida, IEEE Electron Device Lett. 7, 404 (1986)

192. J.W. Palmour, H.S. Kong, R.F. Davis, Appl. Phys. Lett. 51, 2028 (1987)

193. T. Nakamura, K. Nanbu, T. Ishikawa, K. Kondo, J. Appl. Phys. 64, 2164 (1988)

194. G. Kelner, M.S. Shur, S. Binari, K.J. Sleger, H.-S. Kong, Trans. Electron Device 36, 1045 (1989)

195. R.F. Davis, G. Kelner, M. Shur, J.W. Palmour, J.A. Edmond, Proc. IEEE 79, 677 (1991)

196. J.-W. Hong, N.-F. Shin, T.-S. Jen, S.-L. Ning, C.-Y. Chang, IEEE Electron Device Lett. 13, $375(1992)$

197. M. Bhatnagar, P.K. McLarty, B.J. Baliga, IEEE Electron Device Lett. 13, 501 (1992)

198. M. Ghezzo, D.M. Brown, E. Downey, J. Kretchmer, W. Hennessy, D.L. Polla, H. Bakhru, IEEE Electron Device Lett. 13, 639 (1992)

199. D.M. Brown, E.T. Downey, M. Ghezzo, J.W. Kretchmer, R.J. Saia, Y.S. Liu, J.A. Edmond, G. Gati, J.M. Pimbley, W.E. Schneider, IEEE Trans. Electron Devices 40, 325 (1993)

200. P.G. Neudeck, D.J. Larkin, J.E. Starr, J.A. Powell, C.S. Salupo, L.G. Matus, IEEE Electron Device Lett. 14, 136 (1993)

201. G.-B. Gao, J. Sterner, H. Morkoç, IEEE Trans. Electron Devices 41, 1092 (1994)

202. D.M. Brown, E. Downey, M. Ghezzo, J. Kretchmer, V. Krishnamurthy, W. Hennessy, G. Michon, Solid-State Electron. 39, 1531 (1996)

203. M. Mehregany, C.A. Zorman, N. Rajan, C.H. Wu, Proc. IEEE 86, 1594 (1998)

204. J.C. Zolper, Solid-State Electron. 42, 2153 (1998)

205. T.P. Chow, V. Khemka, J. Fedison, N. Ramungul, K. Matocha, Y. Tang, R.J. Gutmann, SolidState Electron. 44, 277 (2000)

206. A. Elasser, T.P. Chow, Proc. IEEE 90, 969 (2002)

207. F. Ren, J.C. Zolper (eds.), Wide Energy Bandgap Electronic Devices (World Scientific, River Edge, 2003) 
208. J.H. Zhao, Mater. Res. Soc. Bull. 30, 293 (2005)

209. Q. Zhang, R. Callanan, M.K. Das, S.-H. Ryu, A.K. Agarwal, J.W. Palmour, IEEE Trans. Power Electron. 25, 2889 (2010)

210. K. Schirmer, B. Rowden, H.A. Mantooth, S.S. Ang, J.C. Balda, ECS Trans. 41, 183 (2011)

211. R.S. Pengelly, S.M. Wood, J.W. Milligan, S.T. Sheppard, W.L. Pribble, IEEE Trans. Microw. Theory Techniq. 60, 1764 (2012)

212. L. Lanni, R. Ghandi, B.G. Malm, C.-M. Zetterling, M. Östling, IEEE Trans. Electron. Devices 59, $1076(2012)$

213. H.A. Mantooth, M.D. Glover, P. Shepherd, IEEE J. Emerg. Sel. Topics Power Electron. 2, $374(2014)$

214. H.R. Philipp, Phys. Rev. 111, 440 (1958)

215. V. Grivickas, J. Linnros, P. Grivickas, A. Galeckas, Mater. Sci. Eng. B 61-62, 197 (1999)

216. S. Nakamura, H. Kumagai, T. Kimoto, H. Matsunami, Appl. Phys. Lett. 80, 3355 (2002)

217. W. Bartsch, R. Schoerner, K.O. Dohnke, Mater. Sci. Forum 645-648, 909 (2010)

218. E.A. Burgemeister, W. von Muench, E. Pettenpaul, J. Appl. Phys. 50, 5790 (1979)

219. D.L. Barrett, R.G. Seidensticker, W. Gaida, R.H. Hopkins, W.J. Choyke, J. Cryst. Growth 109, 17 (1991)

220. R. Mickevičius, J.H. Zhao, J. Appl. Phys. 83, 3161 (1998)

221. J. Wu, W. Walukiewicz, K.M. Yu, J.W. Ager III, E.E. Haller, H. Lu, W.J. Schaff, Y. Saito, Y. Nanishi, Appl. Phys. Lett. 80, 3967 (2002)

222. S.X. Li, J. Wu, E.E. Haller, W. Walukiewicz, W. Shan, H. Lu, W.J. Schaff, Appl. Phys. Lett. 83, 4963 (2003)

223. W. Walukiewicz, Physica E 20, 300 (2004)

224. K. Bejtka, F. Rizzi, P.R. Edwards, R.W. Martin, E. Gu, M.D. Dawson, I.M. Watson, I.R. Sellers, F. Semond, Phys. Status Solidi A 202, 2648 (2005)

225. I. Gorczyca, T. Suski, N.E. Christensen, A. Svane, Appl. Phys. Lett. 96, 101907 (2010) 
226. S.J. Pearton, R. Deist, F. Ren, L. Liu, A.Y. Polyakov, J. Kim, J. Vac. Sci. Technol. A 31, 050801 (2013)

227. S.K. O'Leary, B.E. Foutz, M.S. Shur, L.F. Eastman, Solid State Commun. 118, 79 (2001)

228. I. Akasaki, H. Amano, H. Murakami, M. Sassa, H. Kato, K. Manabe, J. Cryst. Growth 128, 379 (1993)

229. S.N. Mohammad, A.A. Salvador, H. Morkoç, Proc. IEEE 83, 1306 (1995)

230. S. Porowski, J. Cryst. Growth 166, 583 (1996)

231. S.P. Denbaars, Proc. IEEE 85, 1740 (1997)

232. M.S. Shur, Solid-State Electron. 42, 2131 (1998)

233. C. Liu, F. Yun, H. Morkoç, J. Mater. Sci.: Mater. Electron. 16, 555 (2005)

234. M. Bockowski, Cryst. Res. Technol. 42, 1162 (2007)

235. R.P. Davies, C.R. Abernathy, S.J. Pearton, D.P. Norton, M.P. Ivill, F. Ren, Chem. Eng.

Commun. 196, 1030 (2009)

236. R. Brazis, R. Raguotis, Phys. Status Solidi C 6, 2674 (2009)

237. J.A. del Alamo, J. Joh, Microelectron. Reliab. 49, 1200 (2009)

238. A. Katz, M. Franco, IEEE Microw. Mag. 11, S24 (2010)

239. M. Razeghi, IEEE Photonics J. 3, 263 (2011)

240. Y. Hao, J. Zhang, B. Shen, X. Liu, J. Semicond. 33, 081001 (2012)

241. B.J. Baliga, Semicond. Sci. Tech. 28, 074011 (2013)

242. S. Colangeli, A. Bentini, W. Ciccognani, E. Limiti, A. Nanni, IEEE Trans. Electron Devices 60, $3238(2013)$

243. D.W. Runton, B. Trabert, J.B. Shealy, R. Vetury, IEEE Microw. Mag. 14, 82 (2013)

244. D. Visalli, M. Van Hove, P. Srivastava, J. Derluyn, J. Das, M. Leys, S. Degroote, K. Cheng, M. Germain, G. Borghs, Appl. Phys. Lett. 97, 113501 (2010)

245. I.B. Rowena, S.L. Selvaraj, T. Egawa, IEEE Electron Device Lett. 32, 1534 (2011) 
246. B.A. Danilchenko, I.A. Obukhov, T. Paszkiewicz, S. Wolski, A. Jeżowski, Solid State Commun. 144, $114(2007)$

247. K. Jagannadham, E.A. Berkman, N. Elmasry, J. Vac. Sci. Technol. A 26, 375 (2008)

248. S. Nakamura, Mater. Res. Soc. Bull. 22, 29 (1997)

249. M.S. Shur, M.A. Khan, Mater. Res. Soc. Bull. 22, 44 (1997)

250. A.A. Burk Jr., M.J. O’Loughlin, R.R. Siergiej, A.K. Agarwal, S. Sriram, R.C. Clarke, M.F. MacMillan, V. Balakrishna, C.D. Brandt, Solid-State Electron. 43, 1459 (1999)

251. S. Nakamura, S.F. Chichibu (eds.), Introduction to Nitride Semiconductor Blue Lasers and Light Emitting Diodes (Taylor and Francis, New York, 2000)

252. M.A. Khan, J.W. Yang, W. Knap, E. Frayssinet, X. Hu, G. Simin, P. Prystawko, M. Leszczynski, I. Grzegory, S. Porowski, R. Gaska, M.S. Shur, B. Beaumont, M. Teisseire, G. Neu, Appl. Phys. Lett. 76, 3807 (2000)

253. S. Nakamura, S. Pearton, G. Fasol, The Blue Laser Diode: The Complete Story, 2nd edn. (Springer, New York, 2000)

254. M. Umeno, T. Egawa, H. Ishikawa, Mater. Sci. Semicond. Proc. 4, 459 (2001)

255. A. Krost, A. Dadgar, Phys. Status Solidi A 194, 361 (2002)

256. A. Žukauskas, M.S. Shur, R. Gaska, Introduction to Solid-State Lighting (Wiley, New York, 2002)

257. X. Hu, J. Deng, N. Pala, R. Gaska, M.S. Shur, C.Q. Chen, J. Yang, G. Simin, M.A. Khan, J.C. Rojo, L.J. Schowalter, Appl. Phys. Lett. 82, 1299 (2003)

258. A. Jiménez, Z. Bougrioua, J.M. Tirado, A.F. Braña, E. Calleja, E. Muñoz, I. Moerman, Appl. Phys. Lett. 82, 4827 (2003)

259. W. Lu, V. Kumar, E.L. Piner, I. Adesida, IEEE Trans. Electron Devices 50, 1069 (2003) 260. C.L. Tseng, M.J. Youh, G.P. Moore, M.A. Hopkins, R. Stevens, W.N. Wang, Appl. Phys. Lett. 83, 3677 (2003) 
261. J.C. Carrano, A. Zukauskas (eds.), Optically Based Biological and Chemical Sensing for Defense (SPIE, Bellingham, 2004)

262. M.S. Shur, A. Zukauskas (eds.), UV Solid-State Light Emitters and Detectors (Kluwer, Boston, 2004)

263. M. Shur, M. Shatalov, A. Dobrinsky, R. Gaska, in Advances in GaN and ZnO-Based Thin Film, Bulk, and Nanostructured Materials and Devices, Materials and devices series in materials science, ed. by S. Pearton (Springer, Berlin, 2012), pp. 83-120

264. Ü. Özgür, Ya I. Alivov, C. Liu, A. Teke, M.A. Reshchikov, S. Doğan, V. Avrutin, S.-J. Cho, H. Morkoç, J. Appl. Phys. 98, 041301 (2005)

265. D.H. Levy, S.F. Nelson, J. Vac. Sci. Technol. A 30, 018501 (2012)

266. H. Liu, V. Avrutin, N. Izyumskaya, Ü. Özgür, H. Morkoç, Superlattices Microstruct. 48, 458 (2010)

267. C.-K. Yang, K.S. Dy, Solid State Commun. 88, 491 (1993)

268. J.F. Muth, R.M. Kolbas, A.K. Sharma, S. Oktyabrsky, J. Narayan, J. Appl. Phys. 85, 7884 (1999)

269. P.E. Van Camp, V.E. Doren, Solid State Commun. 95, 173 (1995)

270. H. Gómez, T.R. Taylor, D.M. Neumark, J. Phys. Chem. A 105, 6886 (2001)

271. H. Saitoh, W.A. Yarbrough, Diam. Relat. Mater. 1, 137 (1992)

272. M. Yano, M. Okamoto, Y.K. Yap, M. Yoshimura, Y. Mori, T. Sasaki, Diam. Relat. Mater. 9, $512(2000)$

273. C.-X. Wang, G.-W. Yang, T.-C. Zhang, H.-W. Liu, Y.-H. Han, J.-F. Luo, C.-X. Gao, G.-T. Zou, Appl. Phys. Lett. 83, 4854 (2003)

274. F. Boakye, D. Nusenu, Solid State Commun. 102, 323 (1997)

275. P. Lal, S.K. Srivastava, Microelectron. J. 29, 403 (1998)

276. S. Chaure, N.B. Chaure, R.K. Pandey, A.K. Ray, IET Circuits Devices Syst. 1, 215 (2007) 
277. I. Friel, S.L. Clewes, H.K. Dhillon, N. Perkins, D.J. Twitchen, G.A. Scarsbrook, Diam. Relat. Mater. 18, 808 (2009)

278. P. Hess, J. Appl. Phys. 111, 051101 (2012)

279. A.R. Peaker, A. Mottram, J. Phys. D.: Appl. Phys. 5, 803 (1972)

280. P.V. Gatenby, A.K. Kar, M.F. Kimmitt, IEEE J. Quantum Electron. 15, 69 (1979)

281. B.M. Wessels, Electron. Lett. 15, 748 (1979)

282. A. Tosser, M. Arsalane, A. Piel, Opt. Quantum Electron. 8, 69 (1976)

283. N. Hebalkar, A. Lobo, S.R. Sainkar, S.D. Pradhan, W. Vogel, J. Urban, S.K. Kulkarni, J. Mater. Sci. 36, 4377 (2001)

284. C.G. Rodrigues, Eur. Phys. J. B 72, 405 (2009)

285. S. Miwa, K. Kimura, T. Yasuda, L.H. Kuo, S. Jin, K. Tanaka, T. Yao, Appl. Surf. Sci. 107, 184 (1996)

286. V.D. Ryzhikov, L.P. Gal'chinetskii, S.N. Galkin, K.A. Katrunov, E.K. Lisetskaya, Proc. SPIE 3359, 302 (1998)

287. M.A. Abdel-Rahim, M.M. Hafiz, A.E.B. Alwany, Opt. Laser Technol. 47, 88 (2013)

288. H.S. Hajghassem, W.D. Brown, M.M. Luqman, Microelectron. Reliab. 27, 677 (1987)

289. M. Neumann-Spallart, C. Königstein, Thin Solid Films 265, 33 (1995)

290. N.S. Mansour, K.W. Kim, M.A. Littlejohn, J. Appl. Phys. 77, 2834 (1995)

291. M.A. Khan, Q. Chen, C.J. Sun, M. Shur, B. Gelmont, Appl. Phys. Lett. 67, 1429 (1995)

292. J.D. Albrecht, R.P. Wang, P.P. Ruden, M. Farahmand, K.F. Brennan, J. Appl. Phys. 83, 4777 (1998)

293. S. Yoshida, S. Misawa, S. Gonda, J. Vac. Sci. Technol. B 1, 250 (1983)

294. H. Nakayama, P. Hacke, M.R.H. Khan, T. Detchprohm, K. Hiramatsu, N. Sawaki, Jpn. J. Appl. Phys. 35, L282 (1996)

295. C.A. Hurni, J.R. Lang, P.G. Burke, J.S. Speck, Appl. Phys. Lett. 101, 102106 (2012) 
296. N.G. Weimann, L.F. Eastman, D. Doppalapudi, H.M. Ng, T.D. Moustakas, J. Appl. Phys. 83, 3656 (1998)

297. Z.C. Huang, R. Goldberg, J.C. Chen, Y. Zheng, D.B. Mott, P. Shu, Appl. Phys. Lett. 67, 2825 (1995)

298. S. Krishnamurthy, M. van Schilfgaarde, A. Sher, A.-B. Chen, Appl. Phys. Lett. 71, 1999 (1997)

299. A. Matulionis, J. Liberis, L. Ardaravičius, M. Ramonas, I. Matulionienè, J. Smart, Semicond.

Sci. Technol 17, L9 (2002)

300. C. Bulutay, B.K. Ridley, N.A. Zakhleniuk, Phys. Rev. B 68, 115205 (2003)

301. R. Brazis, R. Raguotis, Appl. Phys. Lett. 85, 609 (2004)

302. S. Gökden, Phys. E 23, 198 (2004)

303. B.K. Ridley, W.J. Schaff, L.F. Eastman, J. Appl. Phys. 96, 1499(2004)

304. A.A.P. Silva, V.A. Nascimento, J. Lumin. 106, 253 (2004)

305. C.E. Martinez, N.M. Stanton, A.J. Kent, M.L. Williams, I. Harrison, H. Tang, J.B. Webb, J.A. Bardwell, Semicond. Sci. Technol. 21, 1580 (2006)

306. M. Tas, B. Tanatar, Phys. Status Solidi C 4, 372 (2007)

307. M. Ramonas, A. Matulionis, L.F. Eastman, Semicond. Sci. Technol. 22, 875 (2007)

308. A. Matulionis, J. Liberis, E. Šermukšnis, J. Xie, J.H. Leach, M. Wu, H. Morkoç, Semicond.

Sci. Technol. 23, 075048 (2008)

309. A. Matulionis, J. Liberis, IEE Proc. Circuits Devices Syst. 151, 148 (2004)

310. M. Ramonas, A. Matulionis, J. Liberis, L. Eastman, X. Chen, Y.-J. Sun, Phys. Rev. B 71, $075324(2005)$

311. J.M. Barker, D.K. Ferry, S.M. Goodnick, D.D. Koleske, A. Allerman, R.J. Shul, Phys. Status Solidi C 2, 2564 (2005)

312. L. Ardaravičius, M. Ramonas, O. Kiprijanovic, J. Liberis, A. Matulionis, L.F. Eastman, J.R. Shealy, X. Chen, Y.J. Sun, Phys. Status Solidi A 202, 808 (2005) 
313. Y. Chang, K.Y. Tong, C. Surya, Semicond. Sci. Technol. 20, 188 (2005)

314. S. Yamakawa, S.M. Goodnick, J. Branlard, M. Saraniti, Phys. Status Solidi C 2, 2573 (2005)

315. A. Reklaitis, L. Reggiani, J. Appl. Phys. 97, 043709 (2005)

316. L.F. Eastman, V. Tilak, J. Smart, B.M. Green, E.M. Chumbes, R. Dimitrov, H. Kim, O.S. Ambacher, N. Weimann, T. Prunty, M. Murphy, W.J. Schaff, J.R. Shealy, IEEE Trans. Electron

Devices 48, 479 (2001)

317. C.H. Oxley, M.J. Uren, IEEE Trans. Electron Devices 52, 165 (2005)

318. M. Farahmand, C. Garetto, E. Bellotti, K.F. Brennan, M. Goano, E. Ghillino, G. Ghione, J.D. Albrecht, P.P. Ruden, IEEE Trans. Electron Devices 48, 535 (2001)

319. M.A. Osman, Proc. SPIE 4280, 109 (2001)

320. T. Li, R.P. Joshi, R.D. del Rosario, IEEE Trans. Electron Devices 49, 1511 (2002)

321. C. Sevik, C. Bulutay, IEEE Proc. Optoelectron. 150, 86 (2003)

322. J.D. Albrecht, R. Wang, P.P. Ruden, M. Farahmand, E. Bellotti, K.F. Brennan, Mater. Res.

Soc. Symp. Proc. 482, 815 (1998)

323. J. Edwards, K. Kawabe, G. Stevens, R.H. Tredgold, Solid State Commun. 3, 99 (1965)

324. V.M. Polyakov, F. Schwierz, I. Cimalla, M. Kittler, B. Lübbers, A. Schober, J. Appl. Phys. 106, 023715 (2009)

325. E. Bellotti, B.K. Joshi, K.F. Brennan, J.D. Albrecht, P.P. Ruden, J. Appl. Phys. 85, 916 (1999) 326. T.L. Tansley, C.P. Foley, J. Appl. Phys. 59, 3241 (1986)

327. T. Matsuoka, H. Okamoto, M. Nakao, H. Harima, E. Kurimoto, Appl. Phys. Lett. 81, 1246 (2002)

328. J. Wu, W. Walukiewicz, W. Shan, K.M. Yu, J.W. Ager III, S.X. Li, E.E. Haller, H. Lu, W.J. Schaff, J. Appl. Phys. 94, 4457 (2003)

329. V.M. Polyakov, F. Schwierz, Appl. Phys. Lett. 88, 032101 (2006)

330. Z. Yarar, Phys. Status Solidi B 244, 3711 (2007) 
331. J.S. Thakur, R. Naik, V.M. Naik, D. Haddad, G.W. Auner, H. Lu, W.J. Schaff, J. Appl. Phys. 99, $023504(2006)$

332. F. Nava, C. Canali, C. Jacoboni, L. Reggiani, S.F. Kozlov, Solid State Commun. 33, 475 (1980)

333. L. Reggiani, D. Waechter, S. Zukotynski, Phys. Rev. B 28, 3550 (1983)

334. Y.C. Kao, O. Eknoyan, J. Appl. Phys. 54, 2468 (1983)

335. R.H. Johnson, O. Eknoyan, J. Appl. Phys. 58, 1402 (1985)

336. V.K. Arora, D.S.L. Mui, H. Morkoç, J. Appl. Phys. 61, 4703 (1987)

337. J.H. Zhao, V. Gruzinskis, Y. Luo, M. Weiner, M. Pan, P. Shiktorov, E. Starikov, Semicond.

Sci. Technol. 15, $1093(2000)$

338. M. Hjelm, K. Bertilsson, H.-E. Nilsson, Appl. Surf. Sci. 184, 194 (2001)

339. M. Hjelm, H.-E. Nilsson, A. Martinez, K.F. Brennan, E. Bellotti,J. Appl. Phys. 93, 1099 (2003)

340. R.P. Joshi, D.K. Ferry, Solid-State Electron. 38, 1911 (1995)

341. H.-E. Nilsson, U. Sannemo, C.S. Petersson, J. Appl. Phys. 80, 3365 (1996)

342. F. Gámiz, J.B. Roldán, J.A. López-Villanueva, VLSI Des. 8, 257 (1998)

343. H.-E. Nilsson, M. Hjelm, C. Fröjdh, C. Persson, U. Sannemo, C.S. Petersson, J. Appl. Phys. 86, 965 (1999)

344. M. Hjelm, H.-E. Nilsson, Phys. Scr. T114, 61 (2004)

345. S.K. O'Leary, B.E. Foutz, M.S. Shur, L.F. Eastman, Solid State Commun. 150, 2182 (2010) 346. H. Arabshahi, M.R. Rokn-Abadi, F.B. Bagh-Siyahi, Res. J. Appl. Sci. 5, 215 (2010)

347. N. Fitzer, A. Kuligk, R. Redmer, M. Städele, S.M. Goodnick, W. Schattke, Semicond. Sci. Technol. 19, S206 (2004)

348. A. Ilgaz, S. Gökden, R. Tülek, A. Teke, S. Özçelik, E. Özbay, Eur. Phys. J. Appl. Phys. 55, $30102(2011)$

349. D.R. Naylor, A. Dyson, B.K. Ridley, Solid State Commun. 152, 549 (2012)

350. D.R. Naylor, A. Dyson, B.K. Ridley, J. Appl. Phys. 111, 053703 (2012) 
351. E. Bellotti, F. Bertazzi, S. Shishehchi, M. Matsubara, M. Goano, IEEE Trans. Electron Devices 60, 3204 (2013)

352. S. Dasgupta, J. Lu, Nidhi, A. Raman, C. Hurni, G. Gupta, J.S. Speck, U.K. Mishra, Appl. Phys. Express 6, 034002 (2013)

353. J.-Z. Zhang, A. Dyson, B.K. Ridley, Appl. Phys. Lett. 102, 062104 (2013)

354. J.J. Freedsman, A. Watanabe, Y. Urayama, T. Egawa, Appl. Phys. Lett. 107, 103506 (2015)

355. Z. Kourdi, B. Bouazza, A. Guen-Bouazza, M. Khaouani, Microelectron. Eng. 142, 52 (2015)

356. L. Gao, Opt. Quantum Electron. 47, 1941 (2015)

357. K. Mazumdar, R.K. Ranjan, R. Shankar, A. Sharan, B. Priyadarshini, M. Kundu, A. Ghosal, Superlattices Microstruct. 100, 983 (2016)

358. L. Sang, X. Yang, J. Cheng, L. Jia, Z. He, L. Guo, A. Hu, Y. Xiang, T. Yu, M. Wang, F. Xu, N. Tang, X. Wang, W. Ge, B. Shen, Appl. Phys. Lett. 107, 052102 (2015)

359. G. Santoruvo, A. Allain, D. Ovchinnikov, E. Matioli, Appl. Phys. Lett. 109, 103102 (2016)

360. W.M. Waller, M.J. Uren, K.B. Lee, P.A. Houston, D.J. Wallis, I. Guiney, C.J. Humphreys, S.

Pandey, J. Sonsky, M. Kuball, IEEE Trans. Electron Devices 63, 1861 (2016)

361. S. Wang, H. Liu, H. Zhang, Q. Chen, Appl. Phys. A 122, 578 (2016)

362. Z. Gu, S.L. Ban, D.D. Jiang, Y. Qu, J. Appl. Phys. 121, 035703 (2017)

363. C. Ostermaier, P. Lagger, G. Prechtl, A. Grill, T. Grasser, D. Pogany, Appl. Phys. Lett. 110, $173502(2017)$

364. M. Zhu, M. Qi, K. Nomoto, Z. Hu, B. Song, M. Pan, X. Gao, D. Jena, H.G. Xing, Appl. Phys. Lett. 110, 182102 (2017)

365. S.K. O'Leary, B.E. Foutz, M.S. Shur, L.F. Eastman, J. Mater. Sci.: Mater. Electron. 21, 218 (2010)

366. E. Baghani, S.K. O’Leary, Appl. Phys. Lett. 99, 262106 (2011)

367. W.A. Hadi, P. Siddiqua, S.K. O'Leary, J. Mater. Sci.: Mater. Electron. 25, 5524 (2014)

368. A.A. Grinberg, S. Luryi, IEEE Trans. Electron Devices 45, 1561 (1998) 
369. I.N. Volovichev, J.E. Velázquez-Perez, Yu.G. Gurevich, Solid- State Electron. 52, 1703 (2008)

370. T. Hashimoto, F. Wu, J.S. Speck, S. Nakamura, Jpn. J. Appl. Phys. 46, L889 (2007)

371. D.F. Storm, M.T. Hardy, D.S. Katzer, N. Nepal, B.P. Downey, D.J. Meyer, T.O. McConkie, L. Zhou, D.J. Smith, J. Cryst. Growth 456, 121 (2016)

372. D. Garrido-Diez, I. Baraia, in 2017 IEEE IEEE International Workshop of Control, Measurement, Signals and their Application to Mechatronics. (2017). https://doi.org/10.1109/ ECMSM.2017.7945876

373. Y.B. Kwon, J.H. Je, P. Ruterana, G. Nouet, J. Vac. Sci. Technol. A 23, 1588 (2005)

374. R.A. Oliver, M.J. Kappers, C.J. Humphreys, Appl. Phys. Lett. 89, 011914 (2006)

375. D.C. Look, R.J. Molnar, Appl. Phys. Lett. 70, 3377 (1997)

376. O. Ambacher, J. Smart, J.R. Shealy, N.G. Weimann, K. Chu, M. Murphy, W.J. Schaff, L.F. Eastman, R. Dimitrov, L. Wittmer, M. Stutzmann, W. Rieger, J. Hilsenbeck, J. Appl. Phys. 85, 3222 (1999)

377. O. Ambacher, B. Foutz, J. Smart, J.R. Shealy, N.G. Weimann, K. Chu, M. Murphy, A.J. Sierakowski, W.J. Schaff, L.F. Eastman, R. Dimitrov, A. Mitchell, M. Stutzmann, J. Appl. Phys. 87, $334(2000)$

378. R. Dimitrov, M. Murphy, J. Smart, W. Schaff, J.R. Shealy, L.F. Eastman, O. Ambacher, M. Stutzmann, J. Appl. Phys. 87, 3375 (2000)

379. I. Saidi, H. Mejri, M. Baira, H. Maaref, Superlattices Microstruct. 84, 113 (2015)

380. D.C. Look, D.C. Reynolds, J.R. Sizelove, R.L. Jones, C.W. Litton, G. Cantwell, W.C. Harsch, Solid State Commun. 105, 399 (1998)

381. H. Tokuda, K. Kodama, M. Kuzuhara, Appl. Phys. Lett. 96, 252103 (2010)

382. B. Gelmont, M.S. Shur, J. Appl. Phys. 78, 2846 (1995)

383. J. Hong, J.W. Lee, C.B. Vartuli, J.D. Mackenzie, S.M. Donovan, C.R. Abernathy, R.V. Crockett, S.J. Pearton, J.C. Zolper, F. Ren, Solid-State Electron. 41, 681 (1997) 
384. D.N. Talwar, D. Sofranko, C. Mooney, S. Tallo, Mater. Sci. Eng. B 90, 269 (2002)

385. E.G. Brazel, M.A. Chin, V. Narayanamurti, D. Kapolnek, E.J. Tarsa, S.P. DenBaars, Appl. Phys. Lett. 70, 330 (1997)

386. J.W. Johnson, E.L. Piner, A. Vescan, R. Therrien, P. Rajagopal, J.C. Roberts, J.D. Brown, S. Singhal, K.J. Linthicum, IEEE Electron Device Lett. 25, 459 (2004)

387. D. Ducatteau, A. Minko, V. Hoël, E. Morvan, E. Delos, B. Grimbert, H. Lahreche, P. Bove, C. Gaquière, J.C. De Jaeger, S. Delage, IEEE Electron Device Lett. 27, 7 (2006)

388. S. Arulkumaran, G.I. Ng, S. Vicknesh, H. Wang, K.S. Ang, C.M. Kumar, K.L. Teo, K. Ranjan, Appl. Phys. Express 6, 016501 (2013)

389. F. Medjdoub, B. Grimbert, D. Ducatteau, N. Rolland, Appl. Phys. Express 6, 044001 (2013)

390. S. Arulkumaran, G.I. Ng, S. Vicknesh, IEEE Electron Device Lett. 34, 1364 (2013)

391. K. Ranjan, S. Arulkumaran, G.I. Ng, S. Vicknesh, Appl. Phys. Express 7, 044102 (2014)

392. S. Huang, K. Wei, G. Liu, Y. Zheng, X. Wang, L. Pang, X. Kong, X. Liu, Z. Tang, S. Yang, Q. Jiang, K.J. Chen, IEEE Electron Device Lett. 35, 315 (2014)

393. L. Yang, M. Mi, B. Hou, H. Zhang, J. Zhu, Q. Zhu, Y. Lu, M. Zhang, Y. He, L. Chen, X. Zhou, L. Lv, X. Ma, Y. Hao, IEEE Electron Device Lett. 38, 1563 (2017) 


\section{Appendices}

\section{Appendix A: Published work while at The University of British Columbia}

At the outset of my studies at The University of British Columbia, the electron transport processes that occur within the zinc-blende phases of $\mathrm{GaN}$ and $\operatorname{lnN}$ were poorly understood. Given the important role that electron transport plays in defining the potential of a given material for device applications, it was clear that further work was required if the recognized device potential of the zinc-blende phases of GaN and $\operatorname{InN}$ was to be fully realized.

Recognizing this critical deficiency in the scientific literature, in collaboration with my academic advisor at The University of British Columbia, Dr. Stephen K. O'Leary, I aimed to amerliorate it. First, I assembled all of the scientific articles focused on electron transport within the wurtzite and zinc-blende phases of $\mathrm{GaN}$ and $\ln N$ that were available. Then I identified what aspects of electron transport remained unresolved, with a particular focus on the less understood materials, i.e. the zinc-blende phases of $\mathrm{GaN}$ and $\mathrm{InN}$. A number of publications arose as a consequence of this body of work, the complete list of my publications being tabulated in Table A.1. While most of these papers are focused on the wurtzite and zinc-blende phases of $\mathrm{GaN}$ and InN, some other results were also published during the course of my doctoral studies, and they are also indicated in Table A.1. In the following paragraphs, each published paper, from which the results presented in this thesis is drawn, is described. The extra papers, not related to the wurtzite and zinc-blende phases of $\mathrm{GaN}$ and $\mathrm{InN}$, are not discussed, as they lie beyond the framework of the current analysis.

I started my work in 2014 with the paper "Non-parabolicity and inter-valley transitions within zinc-blende indium-nitride", published in the Journal of Materials Science: Materials in Electronics, in which we studied how the non-parabolicity of the conduction band valley influences the character of the electron transport that occurs within zinc-blende InN. Steady-state 
Table A.1 Publications of Siddiqua et al.

\begin{tabular}{|c|c|}
\hline Year of publication & Published Article \\
\hline 2018 & $\begin{array}{l}\text { 1. P. Siddiqua, W.A. Hadi, M.S. Shur, and S.K. O'Leary, A steady-state and transient analysis of } \\
\text { the electron transport that occurs within bulk wurtzite zinc-magnesium-oxide alloys subjected to high- } \\
\text { fields, MRS Advances, DOI: } 10.1557 / \text { adv.2018.484, } 2018 \text {. } \\
\text { 2. P. Siddiqua and S.K. O'Leary, Electron transport within the wurtzite and zinc-blende phases of } \\
\text { gallium nitride and indium nitride, Journal of Materials Science: Materials in Electronics, Volume } \\
\text { 29, pages } 3511-3567 \text { (2018). }\end{array}$ \\
\hline 2017 & $\begin{array}{l}\text { 3. S.K. O'Leary, P. Siddiqua, W.A. Hadi, B.E. Foutz, M.S. Shur, and L.F. Eastman, Electron } \\
\text { transport within the III-V nitride semiconductors, Chapter 32, Handbook of Electronic and } \\
\text { Photonic Materials, 2nd Edition, S. Kasap and P. Capper (Eds.), Springer, } 2017 . \\
\text { 4. P. Siddiqua, M.S. Shur, and S.K. O'Leary, The electron transport that occurs within wurtzite zinc } \\
\text { oxide and the application of stress, MRS Advances, DOI: 10.1557/adv.2017.348, } 2017 .\end{array}$ \\
\hline 2016 & $\begin{array}{l}\text { 5. P. Siddiqua and S.K. O'Leary, The sensitivity of the electron transport within bulk zincblende } \\
\text { gallium nitride to variations in the crystal temperature, the doping concentration, and the non- } \\
\text { parabolicity coefficient associated with the lowest energy conduction band valley, Journal of } \\
\text { Applied Physics, Volume 120, pages } 095701-1-12,2016 \text {. } \\
\text { 6. P. Siddiqua, M.S. Shur, and S.K. O'Leary, A sensitivity analysis on the electron transport within } \\
\text { zinc oxide and its device implications, MRS Advances, DOI: } 10.1557 / \text { adv. } 2016.274,2016 \text {. } \\
\text { 7. P. Siddiqua and S.K. O'Leary, The steady-state and transient electron transport within bulk zinc- } \\
\text { blende indium nitride: the impact of crystal temperature and doping concentration variations, Journal } \\
\text { of Applied Physics, Volume 119, pages } 095104-1-10,2016 \text {. }\end{array}$ \\
\hline 2015 & $\begin{array}{l}\text { 8. P. Siddiqua, W.A. Hadi, M.S. Shur, and S.K. O'Leary, A } 2015 \text { perspective on the steady-state } \\
\text { and transient electron transport within the wurtzite phases of gallium nitride, aluminum nitride, and } \\
\text { indium nitride: a critical and retrospective review, Journal of Materials Science: Materials in } \\
\text { Electronics, Volume 26, pages } 4475-4512,2015 \text {. } \\
\text { 9. P. Siddiqua, W.A. Hadi, M.S. Shur, and S.K. O'Leary, Is zinc-oxide a potential material for future } \\
\text { high-power and high-frequency electron device applications? Materials Research Society } \\
\text { Symposium Proceedings, Volume 1805, DOI: } 10.1557 / \text { opl.2015.577, } 2015 \text {. } \\
\text { 10. P. Siddiqua, W.A. Hadi, A.K. Salhotra, M.S. Shur, and S.K. O'Leary, Electron transport and } \\
\text { electron energy distributions within the wurtzite and zinc-blende phases of indium nitride: response } \\
\text { to the application of a constant and uniform electric field, Journal of Applied Physics, Volume 117, } \\
\text { pages } 125705-1-7,2015 \text {. }\end{array}$ \\
\hline 2014 & $\begin{array}{l}\text { 11. W.A. Hadi, P. Siddiqua, and S.K. O'Leary, Non-parabolicity and inter-valley transistions within } \\
\text { zinc-blende indium nitride, Journal of Materials Science: Materials in Electronics, Volume } 25 \text {, } \\
\text { pages } 5524-5534,2014 \text {. }\end{array}$ \\
\hline
\end{tabular}


electron transport was the focus of this analysis. This was followed up with a subsequent paper, published in 2015, entitled "Electron transport and electron energy distributions within the wurtzite and zinc-blende phases of indium nitride in response to the application of a constant and uniform electric field", published in the Journal of Applied Physics, in which the transient electron transport response within these materials was studied in a fair amount of detail.

This course of analysis was followed up with a more thorough examination into the nature of the electron transport that occurs within the zinc-blende phase of indium nitride, published in 2016 in the Journal of Applied Physics, it being entitled "The steady-state and transient electron transport within zinc-blende indium nitride: the impact of crystal temperature and doping concentration variations".

A detailed examination into the nature of the electron transport that occurs within the zincblende phase of the GaN was also presented in 2016 in a paper entitled "The sensitivity of the electron transport within bulk zinc-blende gallium nitride to variations in the crystal temperature, the doping concentration, and the non-parabolicity associated with the lowest energy conduction band valley", which was also published in the Journal of Applied Physics.

By the time I had published these final two aforementioned papers in 2016, I recognized that these articles, that I had authored along with my supervisor, formed the state-of-the-art in terms of what was currently known in terms of electron transport within the zinc-blende phases of GaN and InN; see Figures A.1 and A.2, in which the overall number of articles published on the electron transport within these materials is contrasted with that corresponding to those of the group of O'Leary et al. and those with me, i.e., my publications are, of course, are by definition a sub-set of those of O'Leary et al. The work was completed with the publication of a review article in 2018, entitled "Electron transport within the wurtzite and zinc-blende phases of gallium nitride 


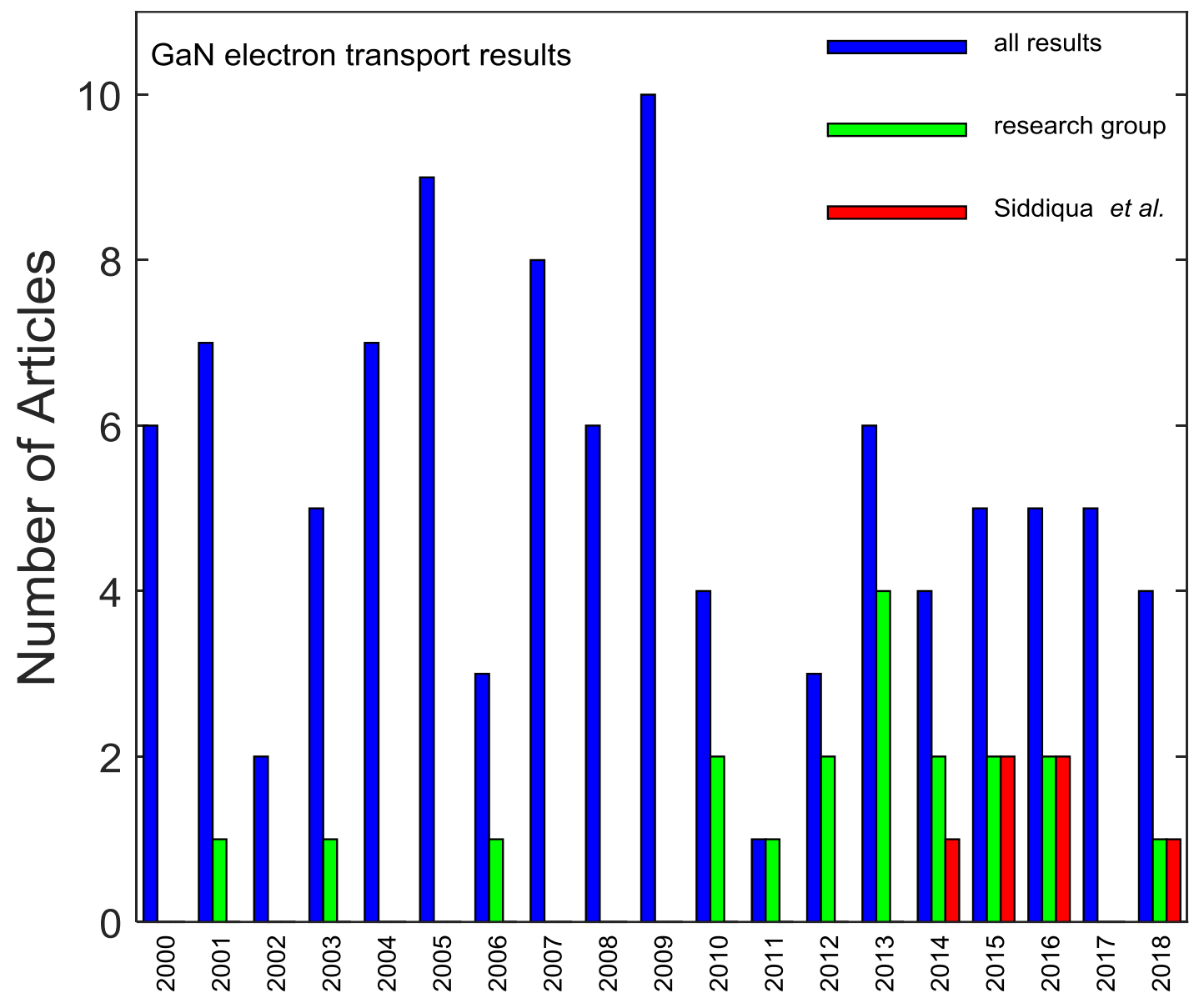

Figure A.1: The number of articles in the field compared with that of O'Leary et al. and Siddiqua et al., for the specific case of GaN. The online version of this figure is depicted in color. 


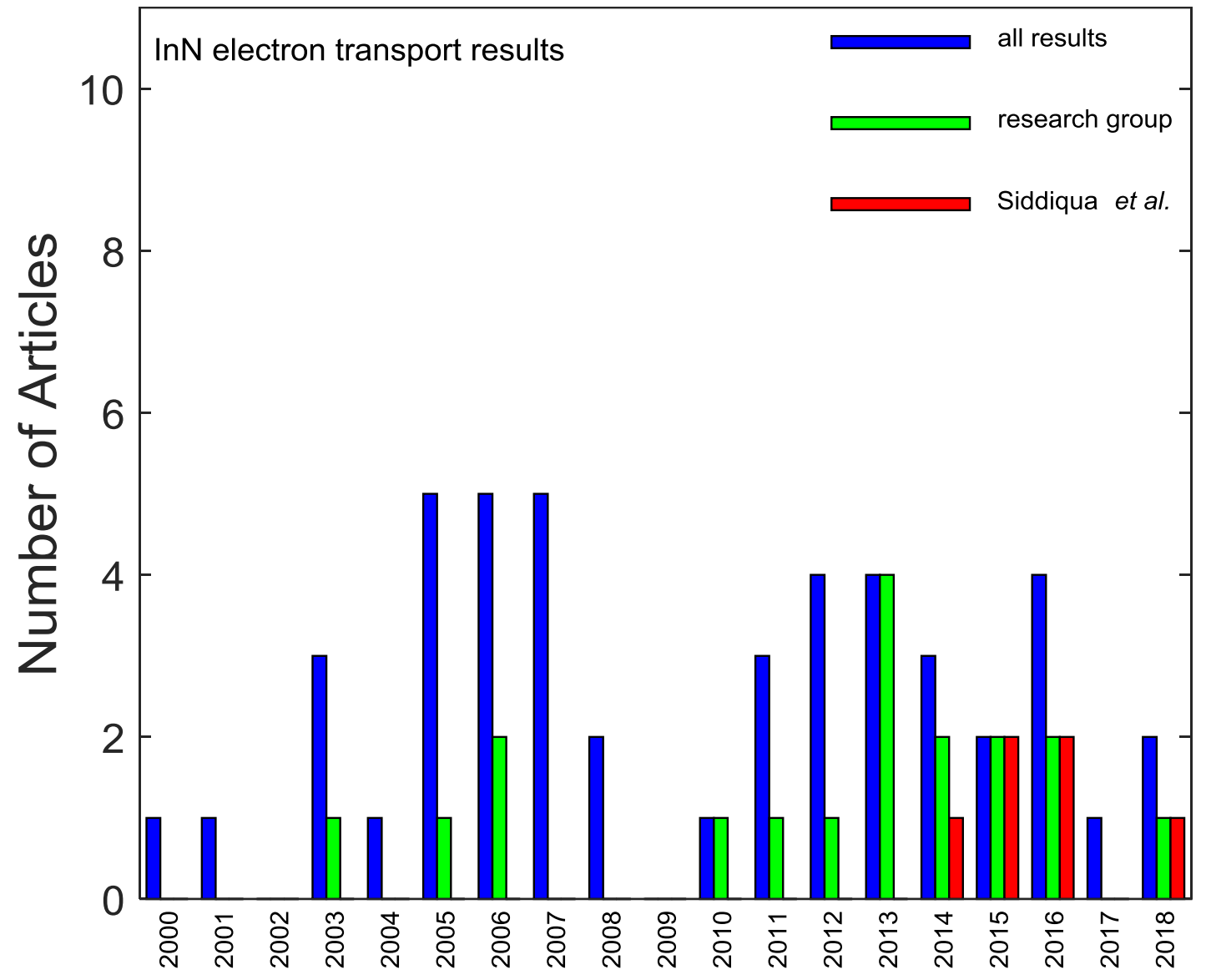

Figure A.2: The number of articles in the field compared with that of O'Leary et al. and Siddiqua et al., for the specific case of $\operatorname{lnN}$. The online version of this figure is depicted in color. 
and indium nitride", which was published in the Journal of Materials Science: Materials in Electronics. This article surveys the state-of-the-art in terms of what is currently known about the electron transport within the wurtzite and zinc-blende phases of $\mathrm{GaN}$ and InN. It features a tutorial on electron transport and the Monte Carlo approach that may be used for its quantitative characterization, a presentation of state-of-the-art electron transport results corresponding to the wurtzite and zinc-blende phases of $\mathrm{GaN}$ and $\mathrm{InN}$, a description of the evolution of the field, and some applications of the results. Potential topics for future research are provided at the conclusion of this review. This review article, in large measure, provides the organizational framework for this thesis. 


\section{Appendix B: The zinc-blende and wurtzite crystal structure}

Some familiar and significant compound semiconductors appear in the zinc-blende and wurtzite crystal structures. Here, I mention some features regarding these two crystal structures.

\section{Zinc-blende Structure}

In a zinc-blende crystal structure, an atom of one kind constituting the binary compound is surrounded by four equidistant atoms of the second kind. In this structure, the two atom types form two interpenetrating face-centered cubic lattices. The zinc-blende structure has tetrahedral coordination. Altogether, the arrangement of atoms in the zinc-blende structure is the same as the diamond cubic structure, but with alternating types of atoms at the different lattice sites. This crystal structure is depicted in Figure B.1.

\section{Wurtzite Crystal Structure}

The basic arrangement of the atoms in the wurtzite structure is similar to that of a zincblende structure. An atom of one kind is surrounded tetrahedrally by four atoms of the second kind, but the tetrahedrons are so oriented that the location of the atoms fit two interpenetrating close-packed hexagonal lattices. The two lattices have the same axis, but one of them is displaced with respect to the other. The wurtzite structure may, therefore, be considered as the closepacked hexagonal structure with a basis of two atoms. This crystal structure is depicted in Figure B.2. 


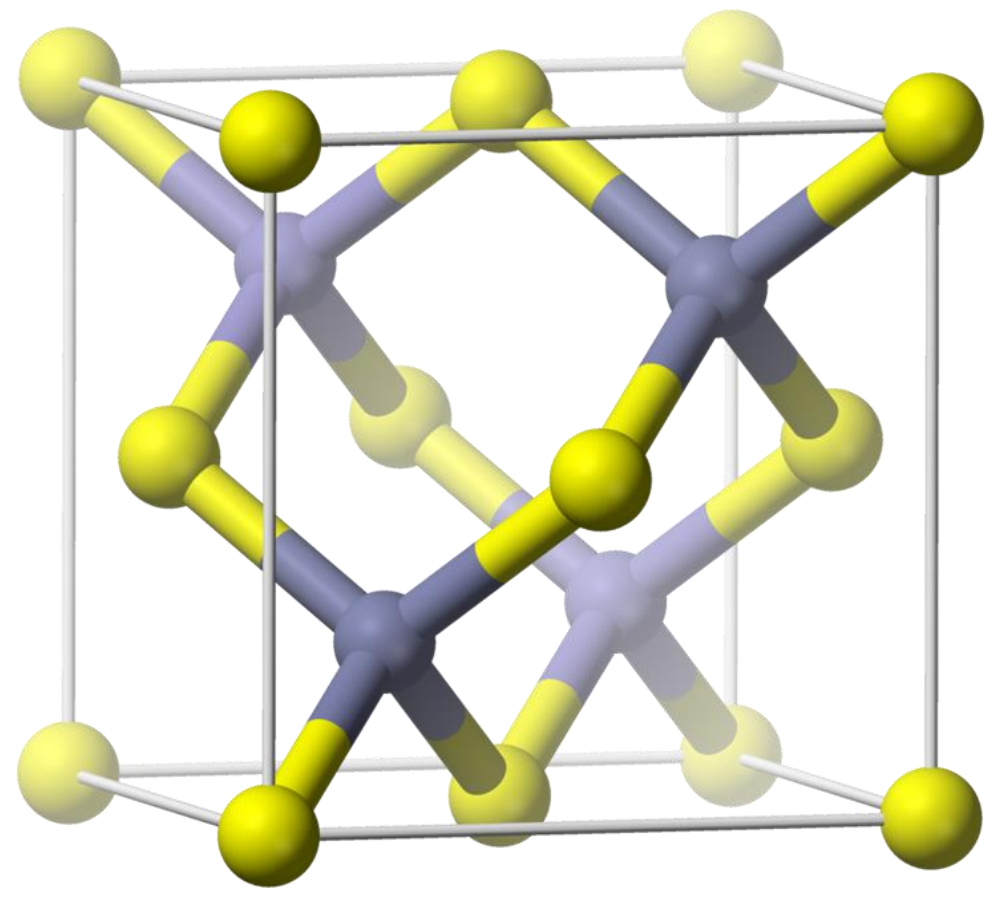

Figure B.1: A zinc-blende unit cell. OBenjah-bmm27, https://en.wikipedia.org/wiki/Cubic crystal system\#/media/File:Sphalerite-unit-cell-depth-fade-3D-balls.png. The online version of this figure is depicted in color. 


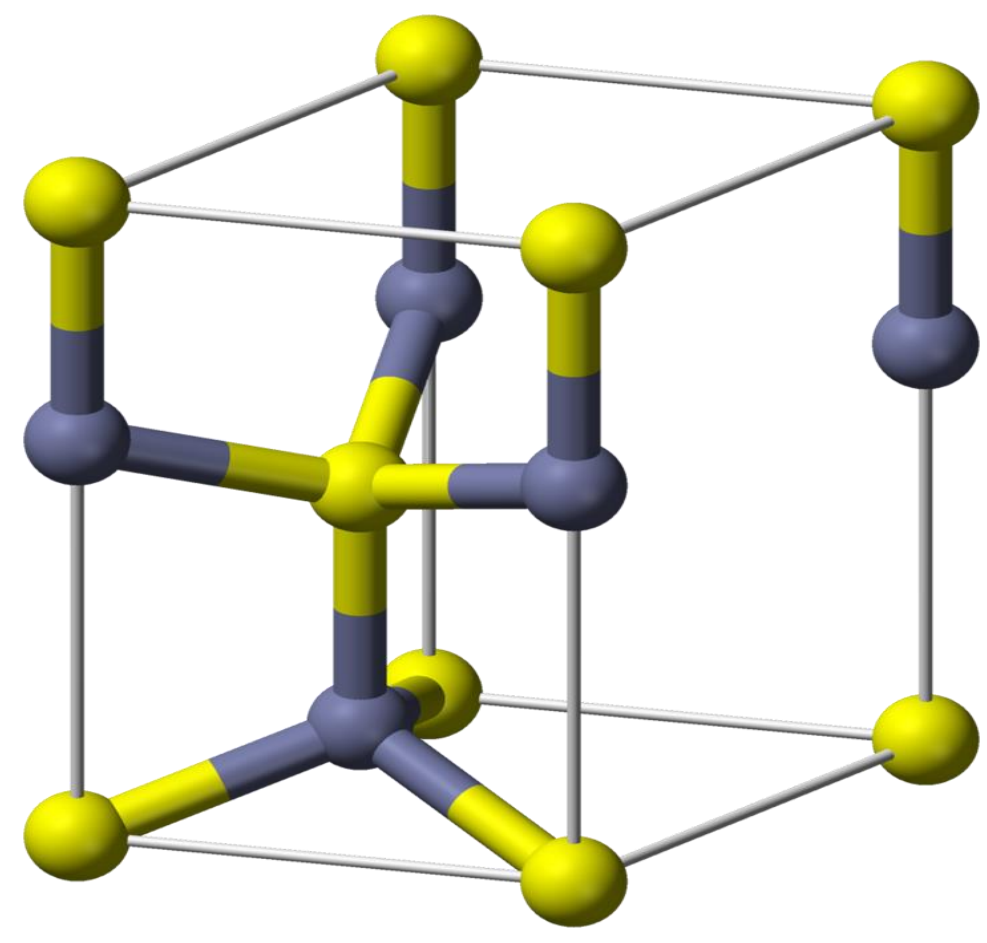

Figure B.2: A wurtzite unit cell. @Benjah-bmm27, https://en.wikipedia.org/wiki/Wurtzite_crystal_structure\#/media/File:Wurtzite-unit-cell-3D-balls.png. The online version of this figure is depicted in color. 


\section{Appendix C: Further details related to my Monte Carlo algorithm}

I now provide further details regarding the semi-classical Monte Carlo algorithm employed for the purposes of my simulations of the electron transport within the III-V nitride semiconductors, GaN and InN. Initially, I overview a more detailed flow chart corresponding to my approach. Then, details of some of the trickier parts of my Monte Carlo algorithm will be discussed. The generation of the free-flight time will then be covered and then the selection of the scattering event (after a free-flight) will be described.

\section{Flow chart for the Monte Carlo algorithm}

A more detailed flow chart for my Monte Carlo algorithm is shown in Figure C.1. This flow chart provides a detailed description of how the dynamics of the electrons are handled, as well as how the statistics are kept during the simulation.

When the simulation initializes, it reads the input file and sets the simulation parameters. Next, the initial electron distribution is determined. During this stage, each electron in the simulation is given an initial wave-vector in accordance with a Maxwell-Boltzmann distribution. At the same time, a rejection technique is used in order to ensure that the number of electrons in any given region of $\vec{k}$ - space never exceeds the Fermi-Dirac limit. This technique provides a close approximation to an initial Fermi-Dirac distribution.

Next, the electric field is set and the scattering rate tables are initialized. The time-step is set to zero and then a loop is entered which moves each particle through free-flights and scattering events until the end of the time-step is reached. After all of the particles are moved, macroscopic quantities, such as the electron drift velocity, are calculated over the distribution and stored in temporary arrays. At the end of the simulation, the accumulated statistics are output to a file. In the next sections, details of some of these steps are provided. 


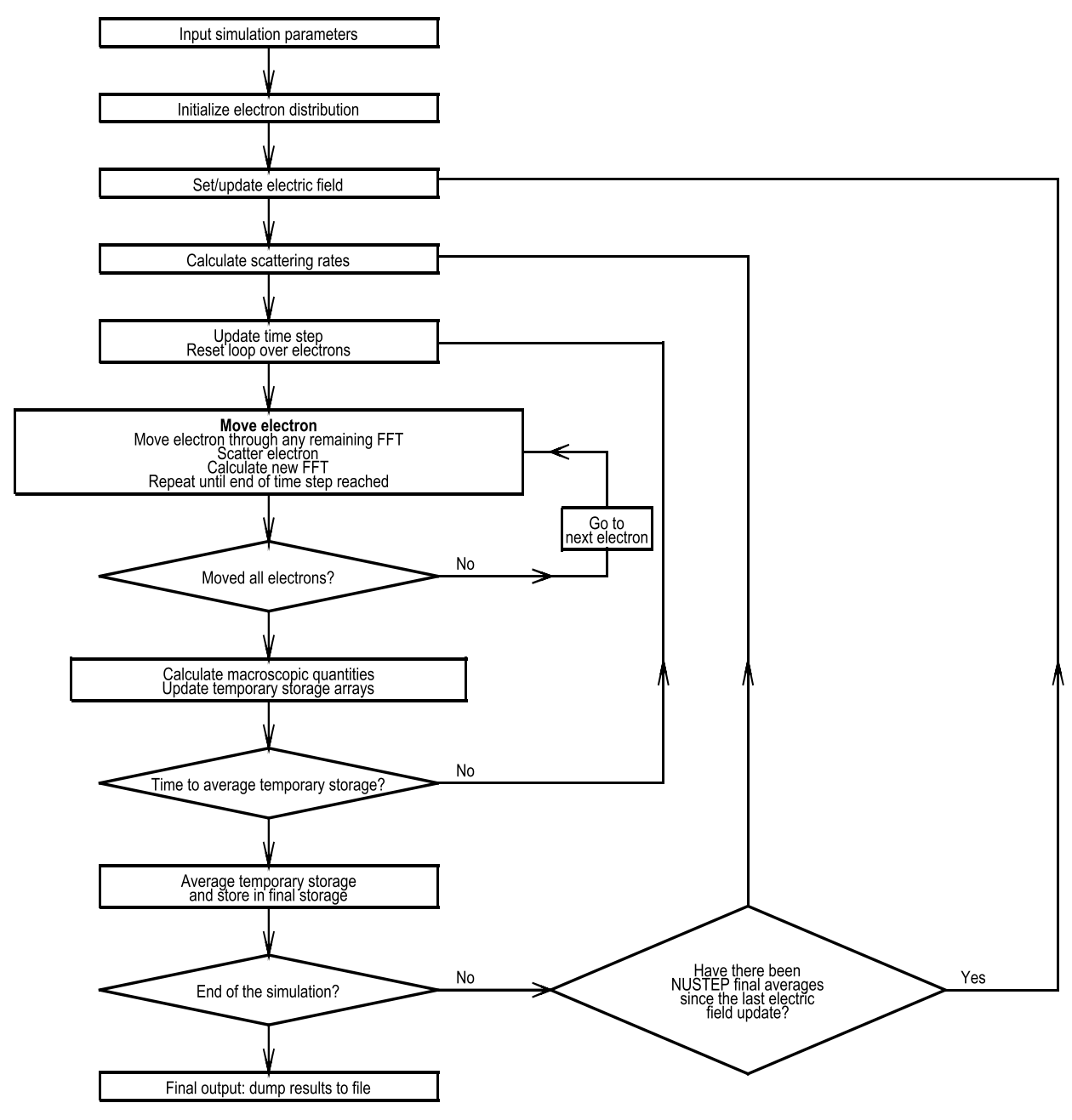

Figure C.1: A more complete flowchart for my Monte Carlo algorithm used for simulating electron transport within the III-V nitride semiconductors, GaN and InN. This figure has been modified from (C Siddiqua, P. \& O'Leary, S.K. (2018). Electron transport within the wurtzite and zinc-blende phases of gallium nitride and indium nitride, Journal of Materials Science, 29, 3511-3567. Page 3557. Adapted with permission from publisher. The online version of this figure is depicted in color. 


\section{Generation of the free-flight times}

The electron energy and its wave-vector, $\vec{k}$, determine the probability that this electron will scatter by means of any of the aforementioned scattering processes. In between each scattering event, the electron's motion is determined through semi-classical physics, i.e., Eqs. (2.4) and (2.5). The amount of time between each scattering event is determined statistically, based on the total scattering rate,

$$
\lambda(\vec{k})=\sum_{i} \lambda_{i}(\vec{k})
$$

which is just the sum of the individual scattering rates corresponding to each scattering mechanism. The statistically determined time between scattering events is known as the freeflight time, $t_{f}$

Generating a proper distribution of free-flight times is essential in order to obtain correct simulation results. A number of methods, used for the purposes of generating these free-flight times, have already been studied in detail [167]. A derivation of the algorithm used in our simulations of the electron transport within the III-V nitride semiconductors, GaN and InN, will be provided here.

We first note that the probability distribution, $P(t)$, for the free-flight time, of length $t$, is just the probability that an electron survives without a collision to time $t$ multiplied by the probability of a collision within a small interval, $d t$, around $t$. The probability of a collision within $d t$ of $t$ is simply the product of the scattering rate at time $t$ and $d t$. The first part of the distribution, the probability that the electron survives to time $t$ without a collision, can be found by assuming that the scattering processes are Poisson in nature. For a Poisson process, the probability of no scattering event for any interval, $\delta t=t_{2}-t_{1}$, is $P(N=0)=\exp (-\lambda \delta t)$. If the scattering rate is constant, this would be the distribution we require. However, the scattering rate changes with time as the electron drifts 
under the action of the applied electric field. To take into account the fact that the scattering rates change with time, we divide the interval, $[0, t]$, into $i$ small intervals. The probability, $p_{i}$, that no scattering event occurs, in interval $\mathrm{i}$, is

$$
p_{i}=\exp \left(-\lambda_{i} \delta t\right)
$$

where $\lambda_{i}$ is the scattering rate during interval $i$ and $\delta t$ is the duration of interval $i$. The probability that no scattering event occurs in any of the $i$ intervals, $O$ through $N$, is the product of the probabilities for each interval, i.e.,

$$
\begin{aligned}
& p(t)=\prod_{i=0}^{N} \exp \left[-\lambda_{i} \delta t\right] \\
& =\exp \left(-\sum_{i=0}^{N} \lambda_{i} \delta t\right) .
\end{aligned}
$$

Letting the intervals become very small, i.e., $\delta t \rightarrow d t$, the sum of Eq. (C.3) reduces to an integral, i.e.,

$$
p(t)=\exp \left[-\int_{0}^{t} \lambda\left(\vec{k}\left(t^{\prime}\right)\right) d t^{\prime}\right]
$$

The free-flight time distribution then becomes the scattering rate multiplied by $p(t)$, i.e.,

$$
P(t)=\lambda(\vec{k}(t)) \exp \left[-\int_{0}^{t} \lambda\left(\vec{k}\left(t^{\prime}\right)\right) d t^{\prime}\right]
$$

In order to generate random free-flight times, with a given $P(t)$, I apply a direct method [76]. In particular, I select a random number, $r$, with a uniform distribution between $[0,1]$, and set it equal to the integrated probability distribution function, i.e.,

$$
r=\int_{0}^{t} P\left(t^{\prime}\right) d t^{\prime}
$$


Substituting Eq. (C.5) into Eq. (C.6), and solving the integral, yields

$$
r=1-\exp \left[-\int_{0}^{t} \lambda\left(\vec{k}\left(t^{\prime}\right)\right) d t^{\prime}\right] .
$$

Thus, we conclude that

$$
-\ln (1-r)=\int_{0}^{t} \lambda\left(\vec{k}\left(t^{\prime}\right)\right) d t^{\prime} .
$$

A time, $t$, must be found which satisfies the above equation for the random number, $r$.

One difficulty in evaluating the integral over $\lambda$ is that it is a complicated function of $t$. This problem can be overcome by introducing an artificial scattering mechanism, known as the selfscattering mechanism, $\lambda_{0}(\vec{k})$. This new mechanism makes the total scattering rate constant over some interval of time, i.e.,

$$
\Gamma=\lambda_{0}(\vec{k})+\lambda(\vec{k}) .
$$

Yorston [167] discusses several algorithms for generating the free-flight times using this self-scattering concept. One of the most efficient algorithms, and the one employed in our Monte Carlo simulations of the electron transport within the III-V nitride semiconductors, GaN and InN, is the constant time method. In this method, a fixed time, $t_{\text {inc, }}$, is chosen, and the integral in Eq. (C.8) is carried out over intervals of length $t_{\text {inc. }}$ In each interval, a self-scattering mechanism, $\lambda_{0}(\vec{k})$, is added in order to make the total scattering rate constant and greater than $\lambda(\vec{k})$ during the $t_{\text {inc }}$ interval. Figure C.2 illustrates this algorithm. The free-flight time is chosen when the total integral satisfies Eq. (C.8). At that time, $\lambda_{0}(\vec{k})$ and each $\lambda_{i}(\vec{k})$ are used to determine the choice of scattering event. 
In the case a self-scattering mechanism is chosen, special treatment is necessary. The integral for the next free-flight time must continue where the previous one left off. In the example shown in Figure C.2, the integral from $t$ to $4 t_{\text {inc }}$ is first used, then that from $4 t_{\text {inc }}$ to $5 t_{\text {inc }}$ is used, and so on.

\section{Choice of scattering event}

Once the electron finishes its free-flight, it scatters. The choice of the scattering event is also made with a random number. This time, the probability that a particular scattering event is selected is directly proportional to the scattering rates corresponding to that particular mechanism. A random number, $r$, uniformly distributed between $[0,1]$, is chosen, and the scattering mechanism, $i$, which satisfies

$$
S_{i}<r<S_{i+1}
$$

where

$$
S_{i}=\frac{\sum_{j=0}^{i} \lambda_{j}(\vec{k})}{\Gamma}
$$

is selected, where

$$
\Gamma=\sum_{i} \lambda_{i}(\vec{k})
$$

Once the scattering mechanism is selected, the final wave-vector of the electron must be chosen. This selection must, of course, obey conservation of energy. With this requirement, there exists a sphere in $\vec{k}$-space into which the electron is allowed to scatter. Therefore, by determining the angle (azimuthal and polar) from the electron's original direction, I may uniquely select the final wave-vector for the electron, and at the same time select the phonon with which the electron 


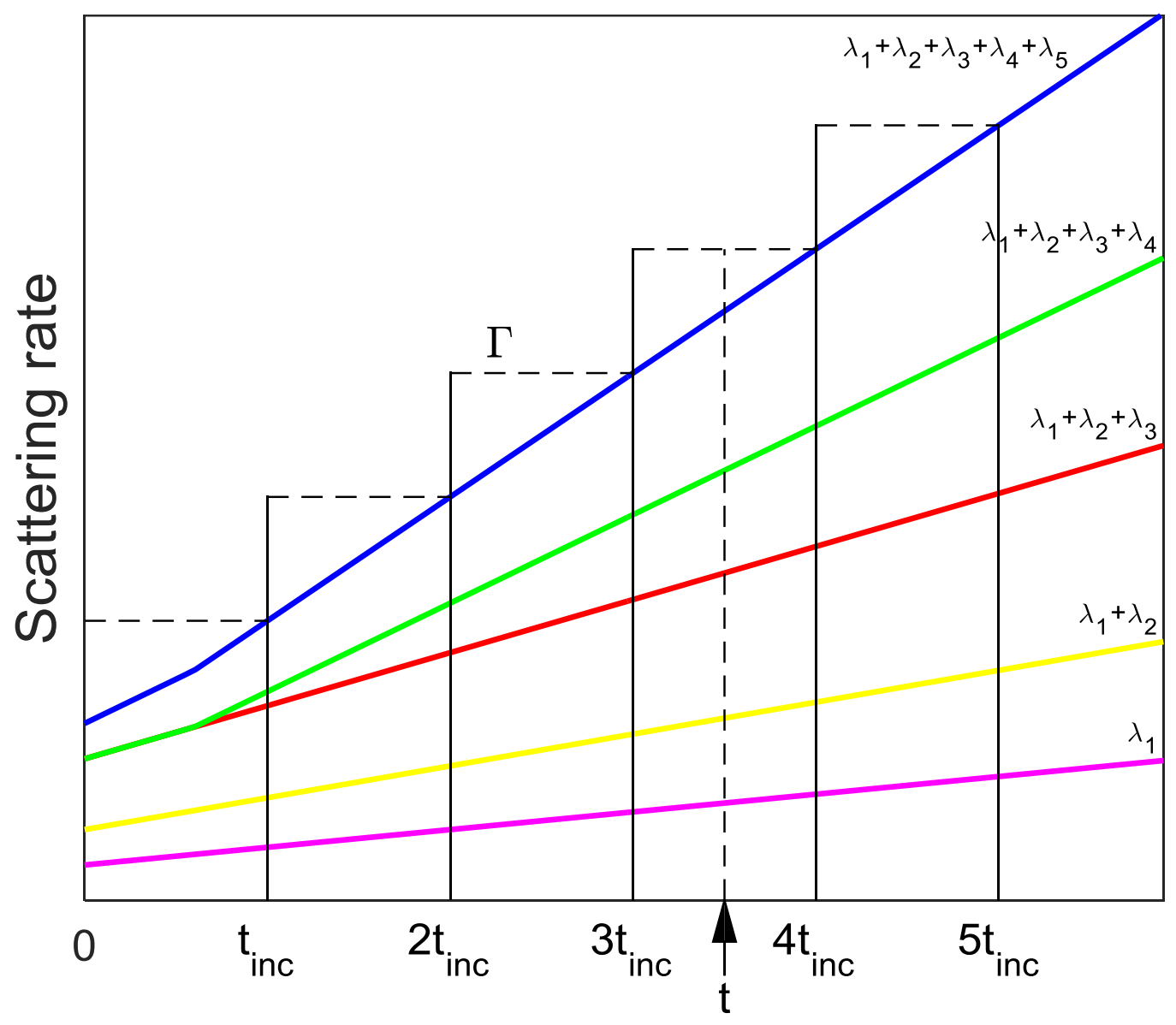

Figure C.2: The scattering mechanism selection process. This figure has been modified from (C) Siddiqua, P. \& O'Leary, S.K. (2018). Electron transport within the wurtzite and zinc-blende phases of gallium nitride and indium nitride, Journal of Materials Science, 29, 3511-3567. Page 3559. Adapted with permission from publisher. The online version of this figure is depicted in color. 
is scattering, in order to obey conservation of momentum considerations. For all the scattering mechanisms selected in our Monte Carlo approach, the selection of the azimuthal angle is done with a uniform distribution, i.e., there is no preference in terms of the azimuthal angle. However, many of the scattering mechanisms have a preference with the polar angle. For each of the scattering mechanisms in the Monte Carlo approach, the dependence of the scattering rate with the polar angle is known, i.e.,

$$
\lambda_{i}(\vec{k})=\int_{0}^{2 \pi} P_{i}(\theta, \vec{k}) d \theta .
$$

There are three different techniques available for converting random numbers with a uniform distribution into one with an arbitrary distribution. These are the direct, rejection, and combined techniques, which are all described by Jacoboni and Lugli [76]. For most of the scattering mechanisms used in my Monte Carlo approach, the rejection technique is used to determine the polar angles. However, some of the most important mechanisms are handled differently. For polar optical phonon and piezoelectric scattering, a combined technique is used. For ionized impurity scattering at low energies, when non-parabolicity can be ignored, the direct technique is used. In other cases, the rejection technique is used, except when the distribution is highly peaked, in which case a combined technique is used.

The simulation continues, moving the electron through each time-step until a special timestep is reached, known as the collection time. After this special time-step, the macroscopic averages, which are stored in temporary arrays, are averaged and stored in permanent arrays. Each average is simply the average over all of the electrons in the simulation. For example, for the electron drift velocity, 


$$
\vec{v}(t)=\frac{\sum v_{i}(t)}{N},
$$

where $N$ denotes the total number of electrons. After each collection time, the scattering rate tables are also recalculated. This occurs because some of the scattering rates, i.e., polar optical phonon, ionized impurity, and piezoelectric, are a function of the electron temperature, which changes throughout the simulation. If the simulation requires that the applied electric field strength to be updated, then it is updated after every fourth collection time (this number can be adjusted). The average from that fourth collection time is assumed to be in steady-state and is associated with the electric field during that interval. At the end of the simulation, the quantities stored in the permanent arrays are written to an output file. 